\title{
Mass Spectrometric Analyses of Post-Translationally Modified Proteins
}

\author{
Dissertation \\ For the Award of the Degree \\ "Doctor of Philosophy" (Ph.D.) \\ Division of Mathematics and Natural Sciences \\ of the Georg-August-Universität Göttingen
}

\author{
Submitted by \\ He-Hsuan Hsiao \\ From Taipei, Taiwan
}

Göttingen 2010 
Members of Thesis Committee

Dr. Henning Urlaub (Instructor)

Bioanalytical Mass Spectrometry Group

Max Planck Institute for Biophysical Chemistry

Prof. Dr. Ralf Ficner (Reviewer)

Department of Molecular Structural Biology

Georg-August-University Göttingen

Prof. Dr. Frauke Melchior (Reviewer)

$\mathrm{ZMBH}$

University of Heidelberg

Date of Oral Examination: August 9, 2010 


\section{Affidavit}

1 hereby declare that this dissertation "Mass Spectrometric Analyses of Post-Translationally Modified Proteins" has been written independently and without unauthorized assistance. This dissertation has not been submitted elsewhere for any academic award or qualification.

He-Hsuan Hsiao

July, 2010

Göttingen, Germany 


\section{Acknowledgement}

First of all, I would like to express my deepest thankfulness to Dr. Henning Urlaub for the opportunity to accomplish my PhD in his laboratory and for funding and supervising this work. Thanks for thinking for me and working wholeheartedly on my manuscripts for publication among his busiest time. He is an greatly kind man and provides many chances to his students for being engaged in their scientific researches.

I thank Prof. Dr. Ralf Ficner of the University of Göttingen and Prof. Dr. Frauke Melchior of University of Heidelberg for serving on my thesis committee, and giving the suggestion of my work.

I am grateful to my colleagues - Uwe Pleßmann, Monika Raabe, Johanna Lehne, Mads Gronborg, Florian Richter, Carla Schmidt, Miroslav Nikolov, Katharina Kramer, llian Atanassov, Romina Hofele and Ling Yun. Your expertises in biology, mass spectrometry and bioinformatics have broaden my horizon. It is a joy working with all of you.

Thanks to my collaborators - Prof. Dr. Reinhard Lührmann and the people in his laboratory who have directly contributed to the phosphoproteomic project in spliceosome; Prof. Dr. Jürgen Wienands and Thomas Oellerich on the SLP 65 project; Prof. Dr. Markus Wahl and Xiao Luo on the NuSB-S10-RNA interaction project; Prof. Dr. Frauke Melchior, Dr. Erik Meulmeester and Benedikt Frank on the SUMO project.

Finally, I would especially like to thank my parent, brothers, girlfriend Chia-Yan Wu and all my friends in Taiwan for being the stone of support that I have built my life upon. I share my success to all of the wonderful people who have supported, assisted, and loved me during my PhD study at University of Götingen. Without these support, I would not have achieved so much. 


\section{Table of Contents}

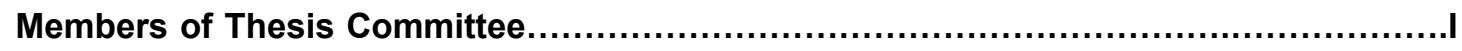

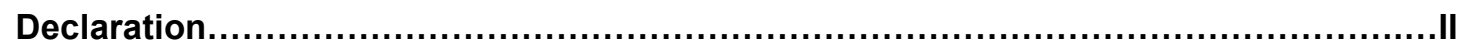

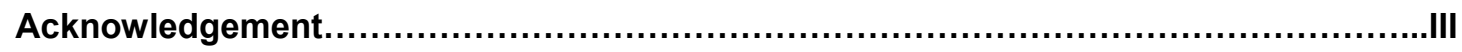

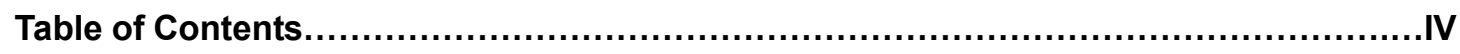

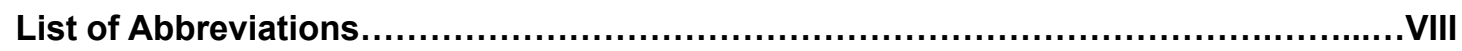

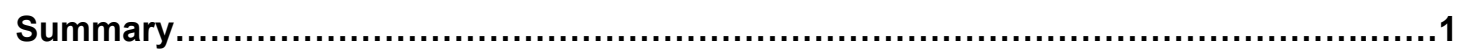

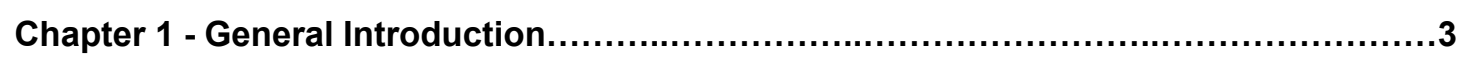

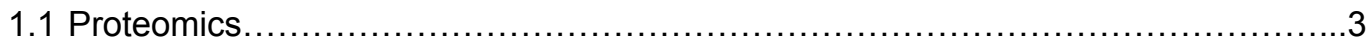

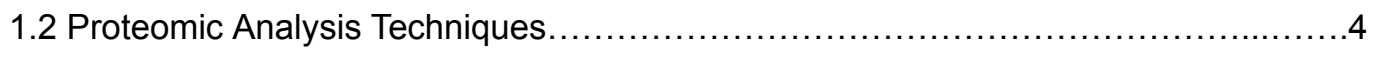

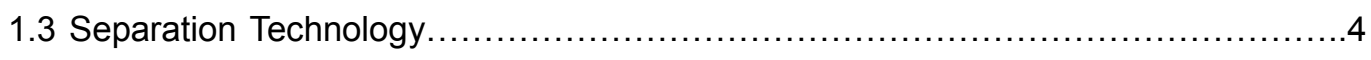

1.3.1 Sodium dodecyl sulfate polyacrylamide gel electrophoresis..................5

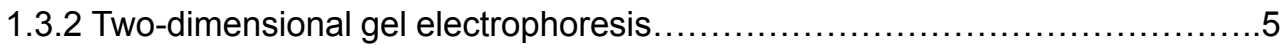

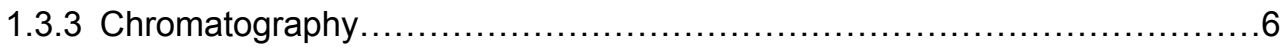

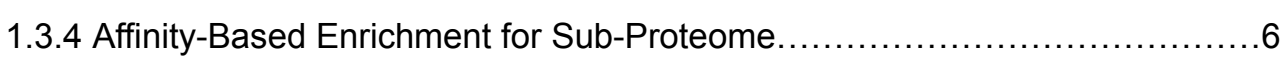

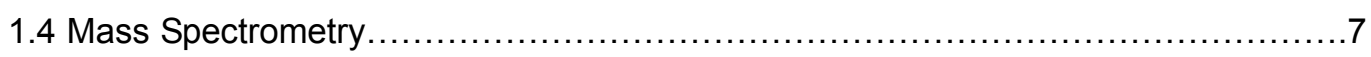

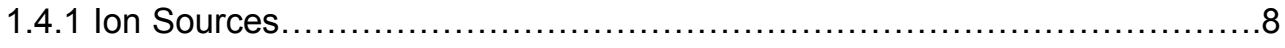

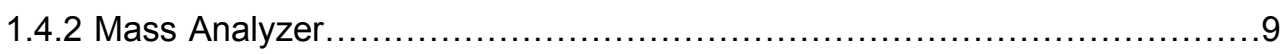

1.4.2.1 Quadrupole Mass Analyzer.....................................10

1.4.2.2 Ion Trap Mass Analyzer............................................ 10

1.4.2.3 Time-Of-Flight Mass Analyzer........................................11

1.4.2.4 Fourier-Transform Ion-Cyclotron Resonance Mass Analyzer...........11

1.4.2.5 Orbitrap Mass Analyzer............................................11

1.4.3 Tandem Mass Spectrometry........................................... 12

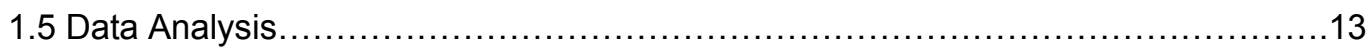

Chapter 2 - A High-Throughput Method for Phosphopeptide Enrichment of

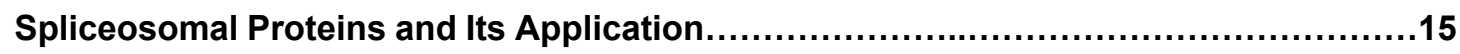

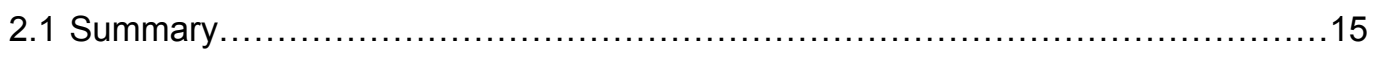

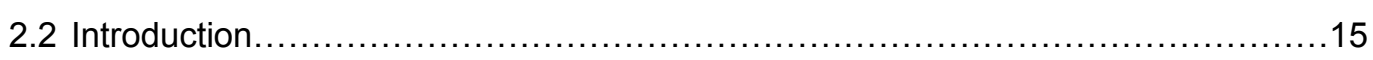

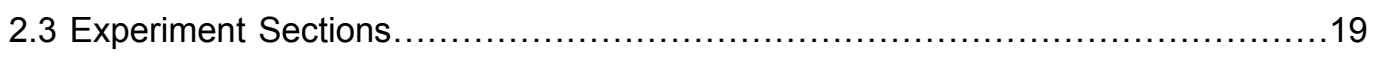

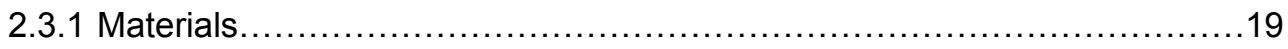

2.3.2 A Crude Mixture of Nuclear snRNP Particles, Individual U snRNP,

Spliceosomal Complexes and SR Proteins Purification...........................19 
2.3.3 Ethanol Precipitation........................................................ 19

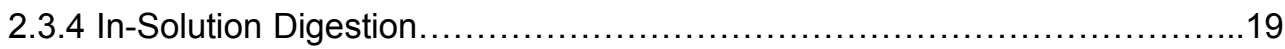

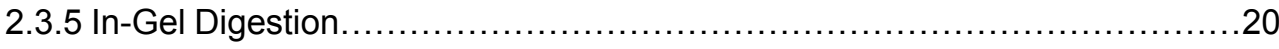

2.3.6 In-House $\mathrm{TiO}_{2}$ Microspin Column Fabrication.............................20

2.3.7 Comparison of Different $\mathrm{TiO}_{2}$ Enrichment Procedure .........................20

2.3.8 NanoLC-ESI and -MALDI Mass Spectrometry Analysis.....................21

2.3.9 Interpretation of Tandem Mass Spectra......................................22

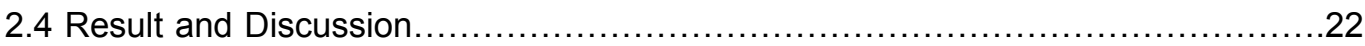

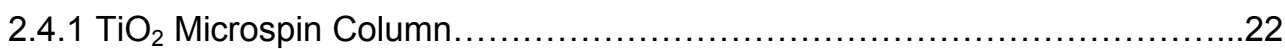

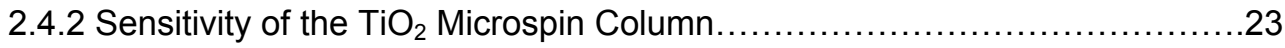

2.4.3 Optimizing Phosphopeptide Enrichment with $\mathrm{TiO}_{2}$ microspin column for

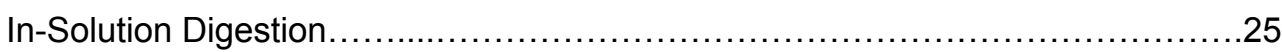

2.4.4 Global Profiling of Phosphopeptides from A Crude Mixture of Nuclear snRNP Particles, Individual U snRNP, Spliceosomal Complexes and SR Proteins.........29

2.4.5 Novel and Known Kinase Motifs in Spliceosomal Proteins.....................34

2.4.6 Application of $\mathrm{TiO}_{2}$ Microspin Column...................................36

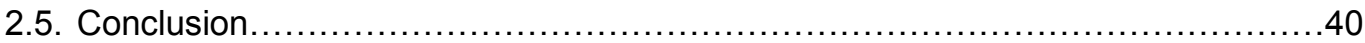

Chapter 3 - Efficient Enrichment of Intact Phosphoproteins prior to Mass Spectrometric

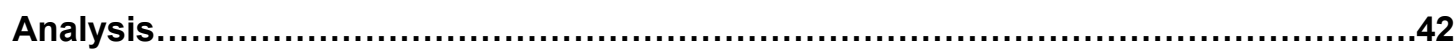

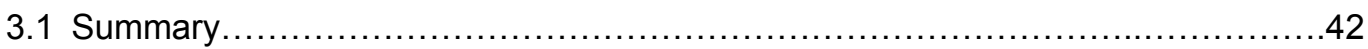

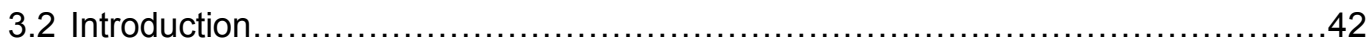

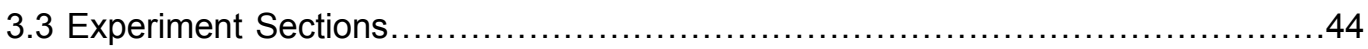

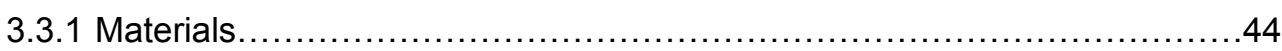

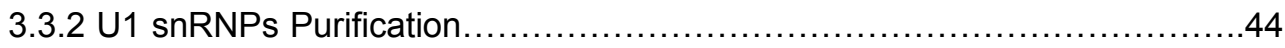

3.3.3 Ethanol Precipitation....................................................... 44

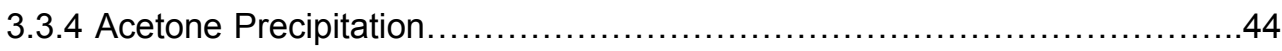

3.3.5 Optimized Calcium Phosphate Precipitation.................................44

3.3.6 In-Solution Digestion........................................................ 45

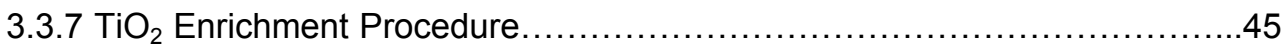

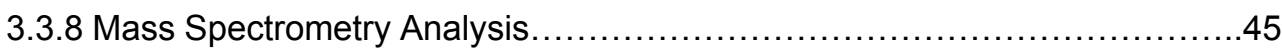

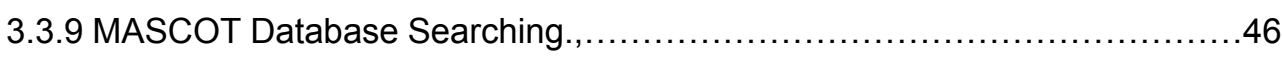

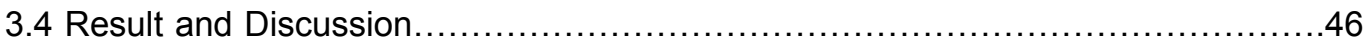

3.4.1 Comparison of Efficiency and Specificity among Different Alkaline Earth Metal

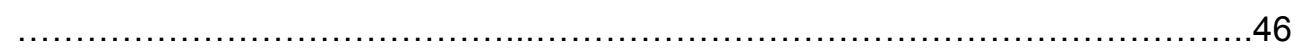

3.4.2 Optimal Condition for Phosphoprotein Precipitation...........................48

3.4.3 Effect of Different Denatured Reagents.................................50

3.4.4 Examine the Power of our Phosphoprotein Isolation Method..................53

3.4.5. Identification of Phosphorylation Sites from U1 Small Nuclear 


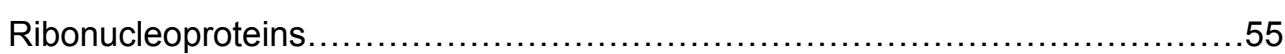

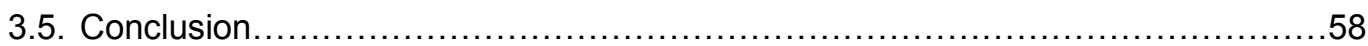

Chapter 4 - Pseudo-Neutral-Loss Scan for Selective Detection of Phosphopeptides and $\mathrm{N}$-Glycopeptides using Liquid Chromatography Coupled with a Hybrid Linear Ion-Trap I

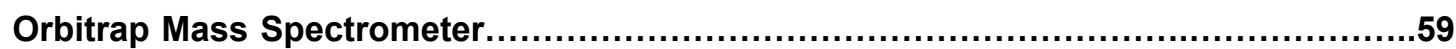

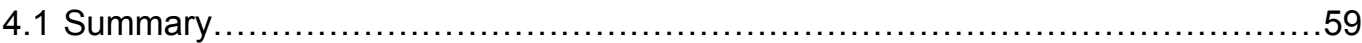

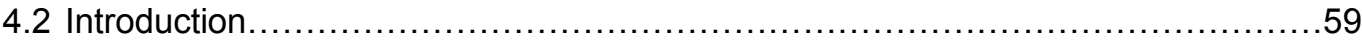

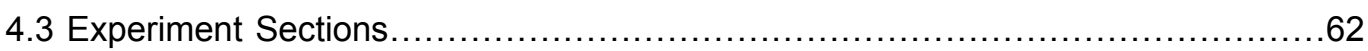

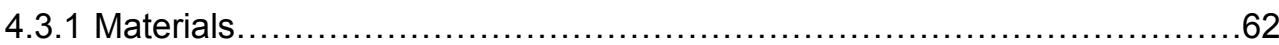

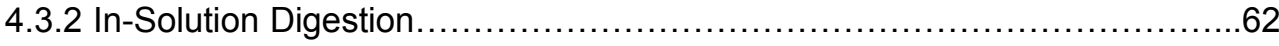

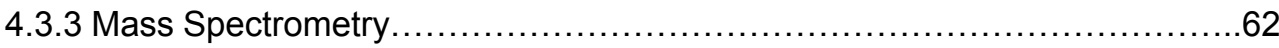

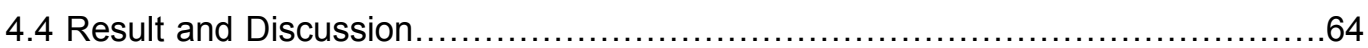

4.4.1 The Principle of Pseudo-Neutral-Loss....................................64

4.4.2 Identification of Phosphopeptide by Pseudo-Neutral-Loss Scan...............65

4.4.3 Identification of $\mathrm{N}$-Glycopeptide by Pseudo-Neutral-Loss Scan...............69

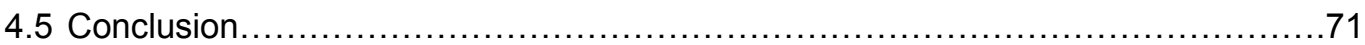

\section{Chapter 5 - "ChopNSpice", a Mass Spectrometric Approach That Allows Identification} of Endogenous Small Ubiquitin-like Modifier-conjugated Peptides...........................73

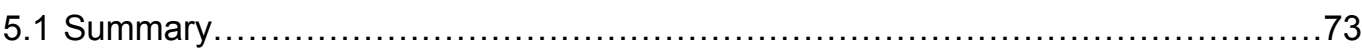

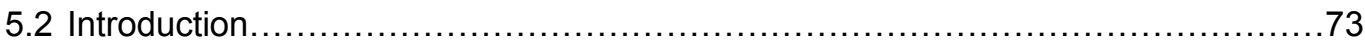

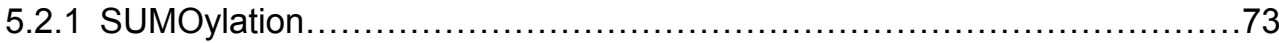

5.2.2 Challenges for Identification of SUMOylation by MS-based

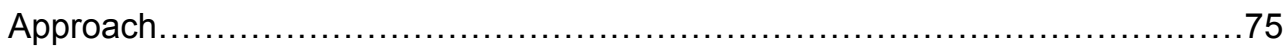

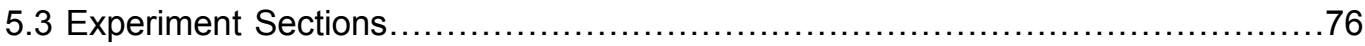

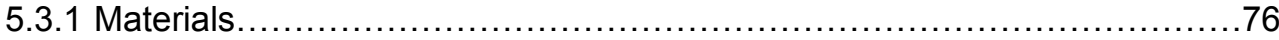

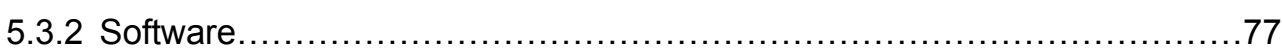

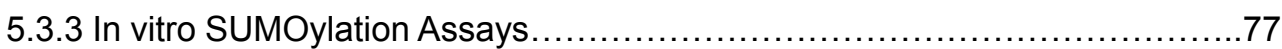

5.3.4 Cell culture, Immunoprecipitation and Immunoblotting.......................77

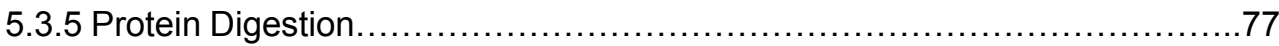

5.3.6 Liquid Chromatography and Mass Spectrometry ...........................78

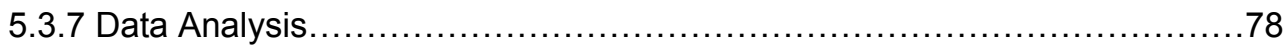

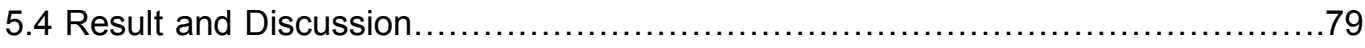

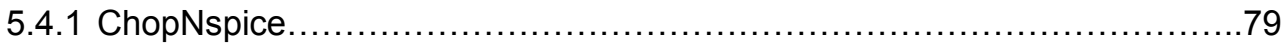

5.4 .2 Identification of SUMO Conjugation Sites in vitro ............................. 84

5.4.3 Increasing Sensitivity by using "High Mass" Acquisition........................86

5.4.4 Identification of SUMO-Conjugated Sites in vivo............................. 87

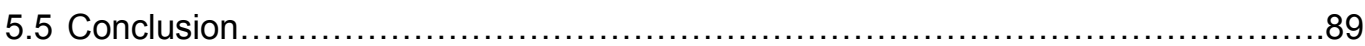




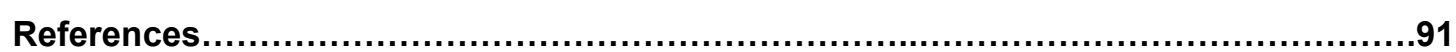

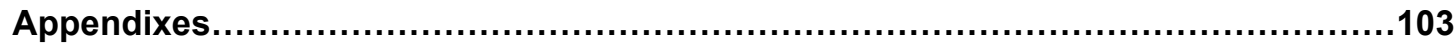

Appendix 1. Phosphorylation sites identified from spliceosomal proteins by using in-house $\mathrm{TiO}_{2}$ microspin column enrichment.

Appendix 2. MS and MS/MS spectra of phosphopeptides derived from human PRP6 and PRP31 120

Appendix 3. Phosphorylation sites identified from U1 snRNPs by using CPP method in combination with in-house $\mathrm{TiO}_{2}$ microspin column enrichment.

Appendix 4A. MASCOT searching result against swissprot bovine database by using regular data-dependent acquisition.

Appendix 4B. MASCOT searching result against swissprot bovine database by using pseudo-neutral-loss acquisition. 


\section{List of Abbreviations}

2DE, two-dimensional gel electrophoresis

$3 \mathrm{D}$ ion trap, three dimensional ions trap

$A C$, alternate current

$\mathrm{ACN}$, acetonitrile

$\mathrm{BCR}$, B-cell receptor

CAA, chloroacetamide

CHCA, $\alpha$-cyano-4-hydroxy-cinnamic acid

CID, collision-induced dissociation

CPP, calcium phosphate precipitation

$\mathrm{DC}$, direct current

DDA, data-dependent acquisition

DeoxyHex, deoxyhexose

DHB, 2,5-dihydroxybenzoic acid

DTT, dithiothreitol

$\mathrm{ESI}$, electrospray ionization

FA, formic acid

FT-ICR, fourier-transform ion-cyclotron resonance

Glu-Fib, [Glu]-Fibrinopeptide B

HEPES, 4-(2-hydroxyethyl)-1-piperazineethanesulfonic acid

Hex, hexose

HexNAc, $\mathrm{N}$-acetylhexosamine

IAA, lodoacetamide

IMAC, immobilized metal ion affinity chromatography

$\mathrm{kDa}$, kilodalton

LC, liquid chromatography

$\mathrm{m} / \mathrm{z}$, mass-to-charge ratio

MALDI, matrix-assisted laser desorption / ionization-time of flight mass spectrometer

$\mu g$, microgram

$\mu \mathrm{L}$, microliter

$\mathrm{mg}$, milligram

min, minute

MOAC, metal oxide affinity chromatography

MS, mass spectrometry

MS/MS, tandem mass spectrometry

MudPIT, multi-dimensional protein identification technology

$\mathrm{nL}$, nanoliter

NeuAc, $N$-acetylneuraminic acid 
NeuGc, N-glycolylneuraminic acid

NusB, $N$ utilization substance protein $B$

p53, cellular tumor antigen p53

PA, phthalic acid

PMF, peptide mass fingerprinting

ppm, parts per million

pre-mRNA, precursor messenger ribonucleic acid

PRP31, pre-mRNA-processing factor 31

PRP6, pre-mRNA-processing factor 6

PTMs, post-translational modifications

pSer, phosphoserine

pThr, phosphothreonine

pTyr, phosphotyrosine

Q-TOF, quadrupole-time-of-flight

RanGAP1, Ran GTPase-activating protein 1

$\mathrm{RF}$, radio frequency

RNA, ribonucleic acid

$\mathrm{RP}$, reverse phase

rpm, rounds per minute

$\mathrm{S} 10,30 \mathrm{~S}$ ribosomal protein $\mathrm{S} 10$

SAC, strong anion exchange

SCX, strong cation exchange

SDS, sodium dodecyl sulphate

SDS-PAGE, sodium dodecyl sulfate polyacrylamide gel electrophoresis

SLP-65, B-cell linker protein

snRNPs, small nuclear ribonucleoproteins

Sp100, nuclear autoantigen Sp-100

SR proteins, serine/arginine-rich proteins

SUMO, small ubiquitin-like modifier

TFA, trifluoroacetic acid

$\mathrm{TiO}_{2}$, titanium dioxide

TOF, time-of-flight

Uba2, SUMO-activating enzyme subunit 2

USP25, ubiquitin carboxyl-terminal hydrolase 25

UV, ultraviolet 


\section{Summary}

Protein post-translational modifications (PTMs) possess key functions in the regulation of various cellular processes. In this thesis, several new technologies are developed to map protein PTMs, containing phosphorylation, glycosylation and SUMOylation.

Five major topics are presented in this thesis. In Chapter 1- General Introduction, describes what is proteomics, the importance of protein PTMs, the main techniques in proteomic analysis, including the principle of technologies for protein and peptide separation, the theory of mass spectrometry (MS) and concept of proteomic data analysis.

In Chapter 2 - A High-Throughput Method for Phosphopeptide Enrichment of Spliceosomal Proteins and Its Application, a disposable $\mathrm{TiO}_{2}$ microspin column is fabricated in-house for enrichment of phosphopeptides. The method offers several advantages, including high-throughput, easy to use, low cost, high selectivity and sensitivity. In combination with different proteomic strategies, 1381 unique phosphorylation sites corresponding to 390 distinct proteins were identified in spliceosomal proteins. We further applied this method to explore the phosphorylation sites on PRP6, PRP31 and SLP65 and to study protein-RNA interaction by UV-induced crosslinking reaction.

\section{In Chapter 3 - Efficient Enrichment of Intact Phosphoproteins prior to Mass}

Spectrometric Analysis, a straightforward and reliable phosphoprotein purification procedure was developed based on calcium phosphate precipitation (CPP). Integration of $\mathrm{TiO}_{2}$ microspin column, a total of 192 unique phosphorylation sites corresponding to 45 distinct proteins were identified from the U1 small nuclear ribonucleoproteins (snRNPs); of these, 59 phosphorylation sites were not reported previously.

\section{In Chapter 4 - Pseudo-Neutral-Loss Scan for Selective Detection of Phosphopeptides} and $\mathrm{N}$-Glycopeptides using Liquid Chromatography Coupled with a Hybrid Linear Ion-Trap / Orbitrap Mass Spectrometer, a pseudo-neutral-loss scan on a hybrid LTQ-Orbitrap MS was built up for selectively probing phosphopeptides and glycopeptides. The presence of known characteristic mass pair (phosphoric acid or monosaccharide residues) in the spectrum during in-source collision-induced dissociation (CID) was selected to trigger MS/MS and multi-stage activation MS3 fragmentation. Our method is compatible with nano-liquid chromatography (nano-LC) for separation of complex peptide mixtures without any further enrichment. The consequent spectra provide peptide sequence identification and modified site assignment as well as information of the glycan structure. 


\section{Summary}

In Chapter 5 - "ChopNSpice", a Mass Spectrometric Approach That Allows Identification of Endogenous Small Ubiquitin-like Modifier-conjugated Peptides, a novel, user-friendly and straightforward database search tool was developed, called "ChopNSpice", to unambiguously determine the mammalian SUMO1 and SUMO2/3 conjugation sites in vitro and in vivo by mass spectrometry in combination with MS-based search engines like MASCOT or Sequest. High mass data dependent acquisition (DDA) is highly suitable for the accurate detection and sequencing of larger peptides and additionally facilitates detection of lower abundant SUMO-conjugates. We demonstrated the power of ChopNSpice software in combination with proteomic strategy, resulting in the identification of 10 SUMOylated proteins corresponding to 17 distinct sites in endogenous Hela-S3 cells. 15 SUMOylated sites were identified in this study appeared to be novel, which may provide a valuable resource to the biological research community. 


\section{Chapter 1 - General Introduction}

\subsection{Proteomics}

The Human Genome Project and its sister projects for other organisms mark the culmination of twentieth-century biology and have been a tremendous success, rapidly building up a new scope of scientific landscape for the century [1,2]. Following in the footsteps of genomics, the next step has been the development of proteomics. The term "proteome" was coined by Marc Wilkins in 1995 to describe the total set of proteins encoded by a genome. The word "proteome" was defined as "the PROTEin complement expressed by a genOME" [3, 4]. Proteomics is the study of proteome and was first coined by Peter James in 1997 to make an analogy with genomics [5]. Proteins, the main carriers of biological activity. The function of protein depends on the precise amino acid sequence, the modifications, the protein concentration, the association with other proteins, and the extracellular environment. Accordingly, the proteomics is concerned with determining protein structure, modifications, protein expression levels, protein-protein interactions, localization, and cellular roles of as many proteins as possible.

Proteins are converted to their mature forms through a complicated succession of post-translational processing and decoration events, namely post-translational modifications, PTMs. Far from being merely decoration, PTMs of proteins control many biological processes, and examining their diversity and individual functions are critical for understanding mechanisms of cell regulation. As many as 300 PTMs of proteins are known to occur physiologically (http://www.unimod.org/). PTMs are covalent processing events that change the properties of a protein by proteolytic cleavage or by addition of a modifying group to one or more amino acids. Many of the PTMs are regulatory and reversible, most notably are protein acetylation, methylation, phosphorylation, ubiquitination, SUMOylation, and etc., which control biological function through a multitude of mechanisms. It is known that many enzymes and receptors are switched "on" or "off" by phosphorylation and dephosphorylation, such as the enzyme, glycogen synthase kinase-3 (GSK-3), which is phosphorylated by protein kinase B (PKB) as part of the insulin signaling pathway [6]. Ubiquitin is added to proteins as a tag that predestines them for proteolytic degradation [7]. Small ubiquitin-like modifier (SUMO) is found to be attached on many eukaryotic nuclear proteins which functions in the regulation of nuclear-cytosolic transport, transcriptional regulation, apoptosis, protein stability, response to stress, and progression through the cell cycle [8].

Despite the great importance of PTMs for biological functions, large scale studies have been hampered by lacking suitable methods. Mass spectrometry (MS) is currently the most versatile technology to directly determine PTMs due to its sensitivity and selectivity. However, the identification of PTMs still remains a substantial challenge owing to the low stoichiometry of 
modified proteins in combination with large amounts of unmodified proteins in biological sample, which interfere the detection in MS. Hence, direct analysis of PTMs requires isolation of the correctly processed proteins in a significant amount followed by MS-based proteomics, we believe this will lead to a great contribution for the study of protein PTMs.

\subsection{Proteomic Analysis Techniques}

The extraordinary achievements of current proteomics are based largely on the successful developments in the fields of "separation technology", "mass spectrometry" and "data analysis". Once joined, the three disciplines provided a powerful tool to study the proteomics shown in Figure 1.1.

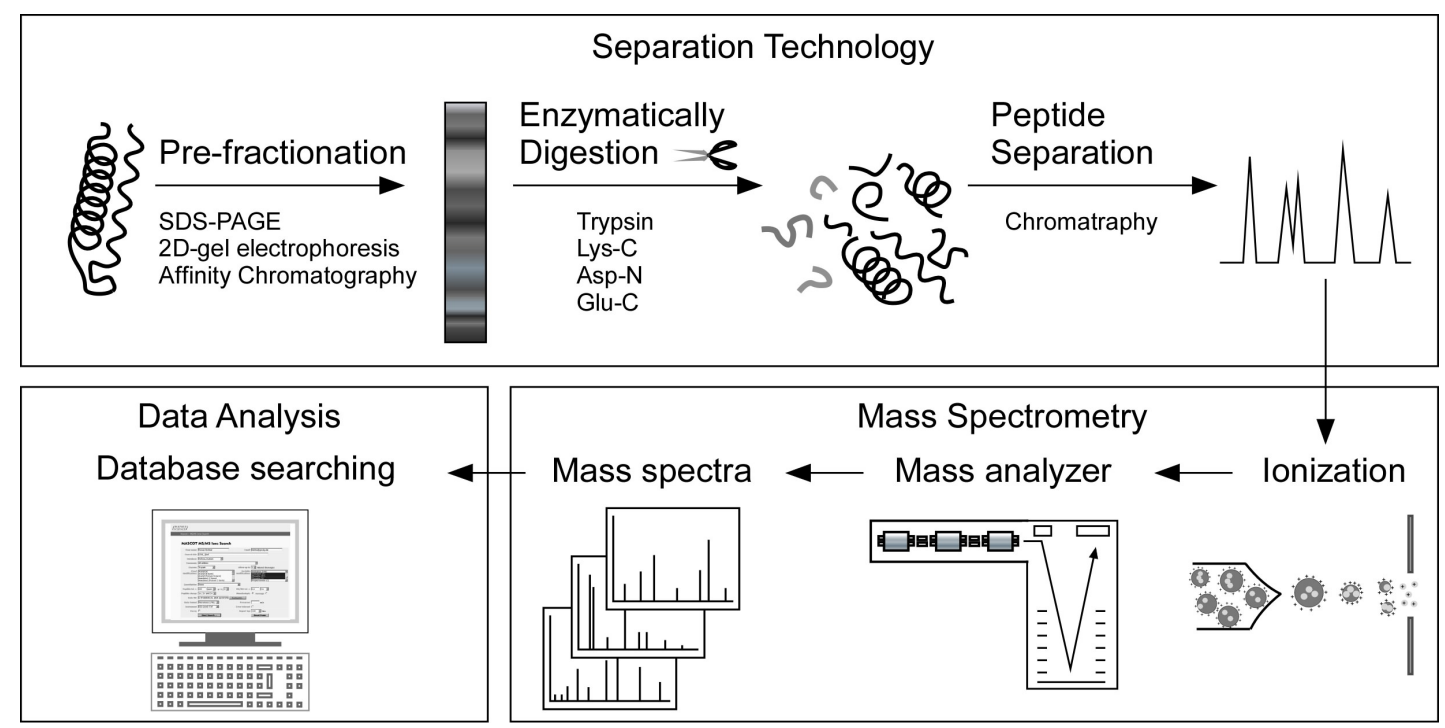

Figure 1.1. Mass spectrometry-based proteomic strategy. The proteins are pre-fractionation, typically separated by SDS-PAGE. The gel lane is cut into several slices, which are then degraded enzymatically into peptides, where C-terminus are protonated amino acids (arginine or lysine) when trypsin is used, providing an advantage in subsequent peptide sequencing. The peptides are separated by one or more steps of chromatographic technologies. The eluted peptides are ionized by either ESI or MALDI. Finally, the resulting mass spectra are searched against protein database to obtain the information of peptide sequences.

\subsection{Separation Technology}

Mass spectrometry-based proteomics is highly dependent and tightly linked to separation technologies that simplify incredibly complex biological samples prior to MS analysis would be crucial. The detection of low abundance species is required front-end separation due to the overshadowed signal of high abundance species. To reduce the complexity, proteins or 
peptides can be resolved into fractions by using various separation methods, including electrophoretic techniques (SDS-PAGE, 2D gel electrophoresis), multi-dimensional chromatography (size exclusion, ion exchange, reverse phase chromatography) and affinity purification.

\subsubsection{Sodium dodecyl sulfate polyacrylamide gel electrophoresis (SDS-PAGE)}

SDS-PAGE is a technique widely used in the separation of proteins according to their electrophoretic mobility (molecular weight) $[9,10]$. The proteins have identical charge per unit mass due to the binding of SDS. Since the charge density is constant, the rate of migration depends on the resistive frictional force, thus small proteins migrate faster than big proteins. The distance traveled in a fixed time period is a log function of the molecular weight.

\subsubsection{Two-dimensional gel electrophoresis}

From the mid 1970s, proteomics was pursued with two-dimensional gel electrophoresis (2DE). The proteins in a sample are separated by isoelectric point and protein mass Each observed protein spot is quantified by its staining intensity. Selected spots are excised, digested and analyzed by mass spectrometer for protein identification shown in Figure 1.2.

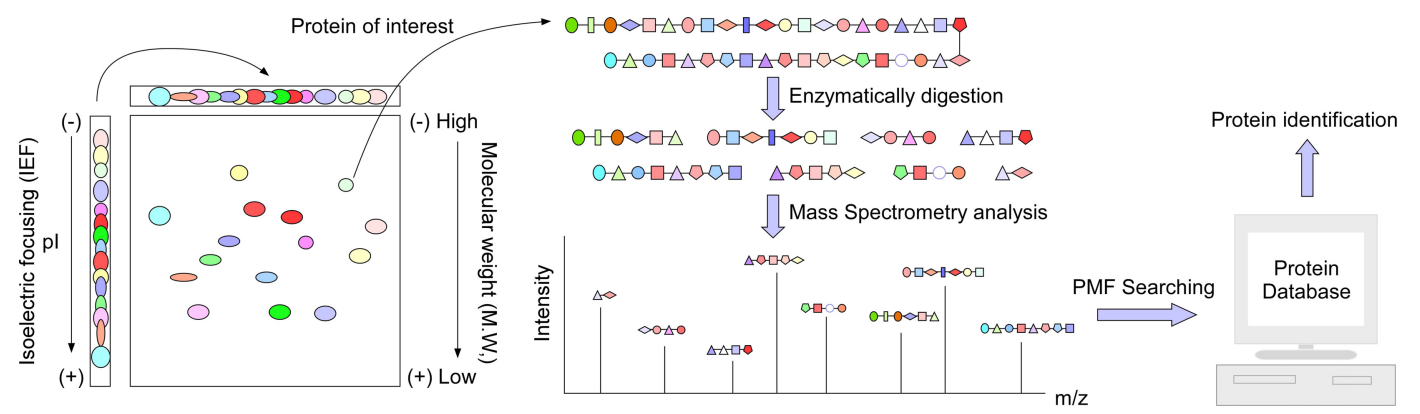

Figure 1.2. Protein identification by two-dimensional gel electrophoresis (2DE) coupling with mass spectrometric peptide mass fingerprinting (PMF). The biological sample is first separated by isoelectric point, called isoelectric focusing (IEF). In the second dimensional separation, an electric potential is again applied, but at a 90 degree angle from the first field, separating the proteins on the basis of their molecular weight. The protein of interest is digested by adequate enzyme, and then analyzed by mass spectrometer. The resulting masses of the peptides of the unknown protein are then compared to the theoretical peptide masses of each protein in the protein database for protein identification, called peptide mass fingerprinting.

2DE has been a mature technique for more than 35 years [11] and was the first technique capable of supporting the concurrent quantitative analysis of large numbers of gene product 
$[12,13]$. Peptide mass fingerprinting (PMF) coupled with matrix-assisted laser desorption / ionization-time of flight mass spectrometer (MALDI-TOF MS) has become highly efficient at the identification of 2DE separated proteins [14-18]. In this method, the unknown protein of interest is first enzymatically digested into peptides, where masses can be accurately measured with a mass spectrometer. These masses are then compared to the theoretical peptide masses of each protein calculated from a known protein database. The results are statistically analyzed to find the best match. For PMF, the matching of four peptides representing $10 \%$ of the sequence does not constitute a reliable hit and should not be listed as a positive identification. In contrast, six peptides representing $20 \%$ of the sequence may be adequate for a tentative identification. There are several shortcomings for this approach. 2DE has limited dynamic range. The protein sequence has to be present in the database of interest. The presence of a mixture can significantly complicate the analysis and potentially compromise the results. Therefore, protein identification based solely on PMF should no longer be acceptable and must be complemented by tandem mass spectrometry (MS/MS) to achieve sufficient specificity of identification.

\subsubsection{Chromatography}

Gel-based technologies have been traditionally used with off-line MALDI analysis. In contract, the multi-dimensional chromatography is usually directly coupled to on-line electrospray ionization (ESI) analysis, a continuous separation, due to their buffers compatibility with ESI. Two major chromatographic materials are widely used for separating peptide mixtures, a reverse phase (RP) and a strong cation exchange (SCX). The reverse phase material separates proteins or peptides based on their hydrophobicity, given high resolution, efficiency, reproducibility. However, the single dimension of separation might not provide sufficient peak capacity to separate peptide mixtures as complex as those generated by the proteolysis of protein mixtures, for example, total cell lysates. Another material, SCX, is integrated with reverse phase as the part of a two-dimensional chromatography, improving the resolving power of separation based on peptide charges and hydrophobicity interaction. This technique is known as multi-dimensional protein identification technology (MudPIT) [19, 20]. High complexity sample is first loaded onto an SCX, and is eluted in a series of salt concentration steps. Each eluted fraction is loaded onto an RP column either off-line or directly eluted into an ESI mass spectrometer. The MudPIT analysis is subdivided the sample into several independent MS runs, which increases the confidence of protein identification and the dynamic range of the measurement.

\subsubsection{Affinity-Based Enrichment for Sub-Proteome}

Another important separation technology is affinity purification which is often used to isolate proteins with PTMs based on a highly specific biological interaction such as that between 
antigen and antibody, enzyme and substrate, or receptor and ligand. The low stoichiometry of PTMs such as phosphorylation, glycosylation and acetylation requires enrichment steps prior to MS analysis. Antibody-based affinity enrichment is widely used for the detection of PTMs in proteins. The antibody purification approach has been successfully used for global analysis of protein lysine acetylation [21], arginine methylation [22], tyrosine phosphorylation [23] and so on. In addtion, the $\mathrm{N}$-glycosylated proteins can be isolated by lectins [24] and the protein containing phosphotyrosine can also be purified by protein containing Scr homology 2 (SH2) domains [25]. However, highly specific antibodies, receptor and substrate are not always available for PTMs of interest. The other affinity enrichment method is based on chemical derivation of the modifying group that derives a "tag" for affinity purification. For example, the O-phosphorylated residues [26] and O-GIcNAc residues [27] (serine or threonine) can be modified an affinity tag by a beta-elimination / Michael addition reaction. Special issues should be taken care of due to the possible loss of the low abundant peptides with PTMs and false-positive protein identification of side-products of chemical reactions

\subsection{Mass Spectrometry}

By fundamental definition, mass spectrometry is designed to measure the mass-to-charge ratio $(\mathrm{m} / \mathrm{z})$ of gas phase ions. It consists of an ion source that converts analyte molecules into gas phase ions, a mass analyzer that separates the $\mathrm{m} / \mathrm{z}$ of the ionized analytes, and a detector that records the number of ions at each $\mathrm{m} / \mathrm{z}$ value, as shown in Figure 1.3.

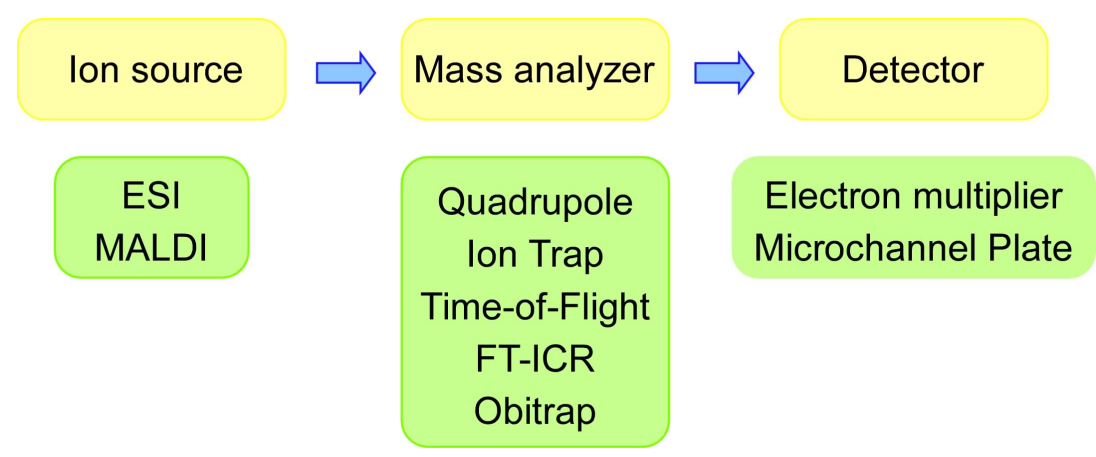

Figure 1.3. Main components of a typical mass spectrometry. Ion source for ion generation, mass analyzer for ion separation and detector to transform analogue signals into digital signals and record a mass spectrum.

Biological mass spectrometry, the technological base of current proteomics studies, was first catapulted to mainstream prominence with the development of ion sources, the electrospray ionization (ESI) [28] and matrix-assisted laser desorption ionization (MALDI) [29-31] techniques. The most notable the discovery and development of protein ionization methods are recognized by the 2002 Nobel Prize in Chemistry (http://nobelprize.org/). Widespread 
mass analyzers are quadrupole, ion trap, time-of-flight (TOF), Fourier-transform ion-cyclotron resonance (FT-ICR) and Orbitrap which can be coupled with either ESI or MALDI ion source.

\subsubsection{Ion Sources}

The development of electrospary ionization (ESI) and matrix-assisted laser desorption ionization (MALDI), the two soft ionization techniques capable of ionizing peptides and proteins, revolutionized protein analysis using MS as shown in Figure 1.4.

(A)

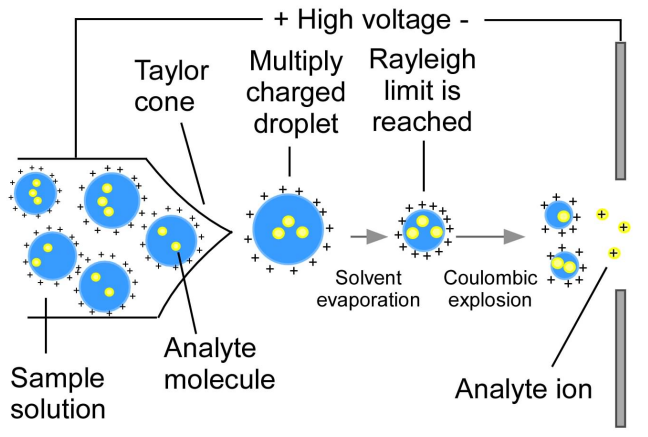

(B)

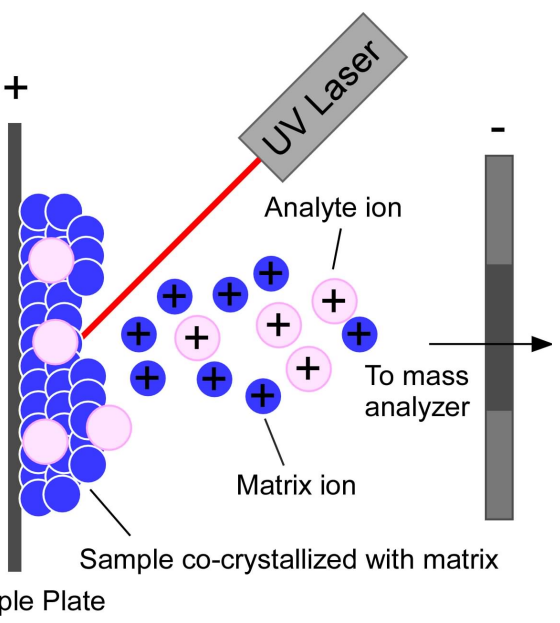

Figure 1.4. The common ionization sources for proteomic research. (A) The scheme of the electrospray ionization (ESI) process. (B) The scheme of matrix-assisted laser desorption ionization (MALDI) process.

The ESI source produces ions from solution at atmospheric pressure shown in Figure 1.4A. ESI is driven by high voltage applied between the end of the capillary column and the inlet of the mass spectrometer. The processes of ESI involve creation of electrically charged spray, Taylor cone [32, 33], followed by formation of an aerosol of charged droplets and desolvation of analyte-solvent droplets. Eventually, ions become free of the solvent that surrounds them, and these ions make their way by voltage-driven into the mass analyzer of the mass spectrometer. The ESI ion formation are the multiply charged species and sensitivity to the analyze concentration and flow rate. Multiply charged ions enable mass spectrometers with limited $\mathrm{m} / \mathrm{z}$ ranges to analyze higher molecular weight molecule. ESI ionizes the analytes out of a solution and is therefore readily coupled to liquid-based (for example, chromatographic and electrophoretic) separation tools.

Unlike ESI, the MALDI source produces ions from solid phase shown in Figure 1.4B. MALDI relies on the utilization of a matrix compound capable of absorbing ultraviolet (UV) light. The matrix and sample are mixed in the appropriate solvent and deposited onto a sample plate. The solvent is evaporated, forming co-crystallized analyte-matrix molecules. MALDI 
sublimates and ionizes the samples out of a dry, crystalline matrix via UV laser pulses. The MALDI matrix absorbs laser energy and transfers the energy to the analyte, whereas the rapid laser heating causes desorption of matrix and analyte ions into gas phase [31,34]. MALDI is normally used to analyze relatively simple samples, whereas ESI integrated with liquid chromatography (LC) is preferred for the analysis of complex samples. Although MALDI can still be coupled to LC, the effluent from LC run must be deposited on a sample plate and mixed with the MALDI matrix, a process that has thus far proven difficult to automate [35].

\subsubsection{Mass Analyzer}

The mass analyzer is, literally and figuratively, central to mass spectrometry. For proteomics research, five basic types of mass analyzers are commonly used: quadrupole, ion trap, time-of-flight (TOF), Fourier-transform ion-cyclotron resonance (FT-ICR) and Orbitrap mass analyzer shown in Figure 1.5. They are very different in design and performance, each with its own strength and weakness. These analyzers can be stand alone or, in some cases, put together in tandem to take advantage of strengths of each. In the context of proteomics, key parameters of mass analyzer are sensitivity, resolution, mass accuracy and the ability to generate information-rich ion mass spectra from peptide fragment (tandem mass or MS/MS spectra).

(A)

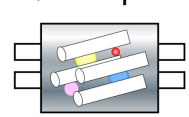

(B)

Reflector TOF-TOF

(D)

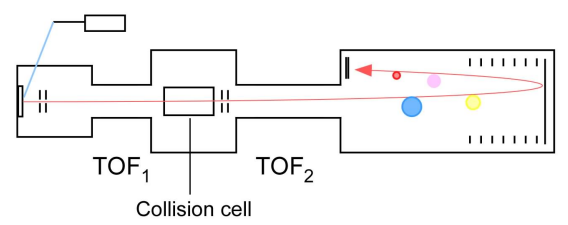

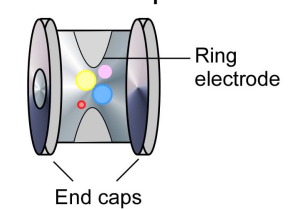

3D ion trap

$(\mathrm{E})$

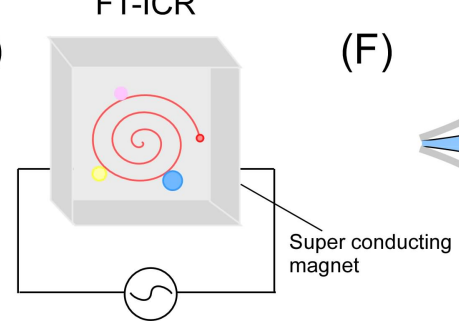

Triple quadrupole

(C)

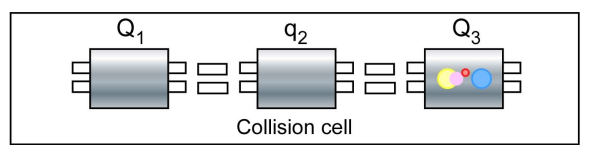

Collision cell

Orbitrap

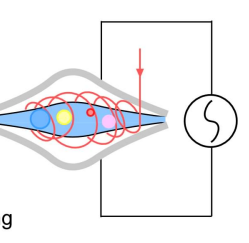

Figure 1.5. Mass spectrometers used in proteomic research. (A) Quadrupole mass spectrometer, the ions are separated by time varying electric fields between four rods, permitting a stable trajectory only for the ions of a particular desired $\mathrm{m} / \mathrm{z}$. (B) Three-dimensional ion trap mass spectrometer, the ions maintain a stable trajectories inside the device as a result of the application of a radio frequency voltage to the ring electrode. Mass analysis is achieved by making ion trajectories unstable in a mass-selective manner. (C) Triple quadrupole mass spectrometer, the ions of a particular 
$\mathrm{m} / \mathrm{z}$ are selected in a first quadrupole $\left(Q_{1}\right)$, fragmented in a collision cell $\left(\mathrm{q}_{2}\right)$, and the fragment ions are separated in the last quadrupole $\left(Q_{3}\right)$. (D) TOF-TOF mass spectrometer, the ions of different $\mathrm{m} / \mathrm{z}$ values have different velocities and therefore reach the detector at different times. It incorporates a collision cell between two TOF sections. Ions of one $\mathrm{m} / \mathrm{z}$ are selected in the first TOF, fragmented in the collision cell, and the fragment ions are separated in the second TOF. (E) Fourier-transform ion-cyclotron resonance (FT-ICR) mass spectrometer, the ions oscillate around the magnetic field at frequencies that are related to their $\mathrm{m} / \mathrm{z}$. As ions oscillate near the top and bottom metal plates of the cubic trapping cell, they induce an alternating current that can be measured and then transferred to $\mathrm{m} / \mathrm{z}$. (F) Orbitrap mass spectrometer, the ions are trapped in its static electrostatic fields, in which the ions orbit around a central electrode and oscillate in axial direction, converting time domain signal into $\mathrm{m} / \mathrm{z}$ like FT-ICR.

\subsubsection{Quadrupole Mass Analyzer}

The quadrupole is a mass filter, consisting of four rods to which direct current $(\mathrm{DC})$ and a radio frequency (RF) alternate current $(A C)$ are applied. Only ions carry a certain $\mathrm{m} / \mathrm{z}$ can pass through, and reach the detector; while others are on unstable trajectories and fail to reach the detector. When these DC and RF voltages are increased, maintaining their ratios constant, ions with increasing value of $\mathrm{m} / \mathrm{z}$ are recorded at the detector $[36,37]$. Up to now, most experiments have been performed on triple quadrupole mass spectrometer that consists of three parts. Two mass separating quadrupoles was divided by a central quadruple whose function is to fragment the selected ion. Due to the presence of two independent quadrupoles, the triple quadrupole can be programmed for a variety of different scan modes, product ion scan, precursor ion scan, neutral loss scan and multiple reaction monitoring [38].

\subsubsection{Ion Trap Mass Analyzer}

The principle of three dimensional ions trap (3D ion trap) is a close relative of the quadrupole mass analyzer. Whereas a quadrupole has electric fields in two dimensions ( $\mathrm{x}$ and $\mathrm{y}$ direction) and the ions move perpendicular to the field ( $z$ direction), the 3D ion trap has the electric field in all three dimensions, which can result in ions being trapped in the field. Unlike quadrupole, the spectrum is obtained by increasing the RF voltage that makes ions unstable and ejects for detection. For a MS/MS acquisition, all ions except the selected ion are ejected first, Subsequently, the remaining ion is fragmented and the product ions are analyzed [39, 40]. Ion trap is a robust, sensitive and relatively inexpensive instrument, which has successfully acquired much proteomics data in the literature. A disadvantage of ion trap is their relatively low mass accuracy due to the limited number of ions that can be accumulated, The space charge in ion trap distorts the accuracy of the mass measurement. Owing to the operating 
principle of the ion trap, the lower end of the fragment mass range cannot be detected ( $1 / 4$ low mass cut off) [40].

\subsubsection{Time-Of-Flight (TOF) Mass Analyzer}

A TOF analyzer separates the ions based on velocity. It can be thought of as a race from the same starting point to the detector. Theoretically, all ions are formed at the same time and place in the ion source. Subsequently, the ions are accelerated to a fixed kinetic energy and travel down a flight tube. The low $\mathrm{m} / \mathrm{z}$ ions achieve higher velocity than the high $\mathrm{m} / \mathrm{z}$ ions. The spectrum is recorded by the impact of each ion on the detector. In fact, ion velocity is inversely related to the square root of $\mathrm{m} / \mathrm{z}[41]$.

\subsubsection{Fourier-Transform Ion-Cyclotron Resonance (FT-ICR) Mass Analyzer}

The FT-ICR mass spectrometer is also a trapping mass analyzer. It captures the ions under high vacuum in a high magnetic field. Once trapped, the ions oscillate with a cyclotron frequency that is inversely related to their $\mathrm{m} / \mathrm{z}$. The trapped ions are excited by an electric RF with a frequency in resonance with their cyclotron frequency. Although the ion oscillation ratio increases, its frequency is maintained, generating the image current for detection. The frequencies related to $\mathrm{m} / \mathrm{z}$ can be calculated by a complex mathematical procedure (Fourier-transform, FT). As the frequencies can be measured precisely, the high-resolution and high-precision mass measurement is achieved under high magnetic field [42].

\subsubsection{Orbitrap Mass Analyzer}

The recent development of a novel Orbitrap mass spectrometry has made the exciting new areas of proteomic application possible. The Orbitrap was invented by Alexander Makarov in 1999 [43] and was reported as a tool for proteomics research in 2005 by Hu et al. [44]. In the Orbitrap analyzer, the ions are trapped and the orbit around a central spindle-like electrode and oscillate harmonically along its axis with a frequency characteristic of their $\mathrm{m} / \mathrm{z}$ values, inducing an image current in the outer electrodes that is processed by Fourier-transform and generates the mass spectrum. The frequency of these harmonic oscillations is independent of the ion velocity and is inversely proportional to the square root of the $\mathrm{m} / \mathrm{z}$. The instrument is capable of mass resolution in excess of 100000 and mass accuracy of less than 2 ppm.

To summarize, MALDI is usually coupled to TOF mass analyzer, whereas ESI has mostly been coupled to ion traps, triple quadrupole, FT-ICR and Orbitrap mass analyzer. More recently, other new combinations of mass spectrometry are developed. For example, ESI quadrupole-TOF (Q-TOF) consists of ESI ion source coupled to the TOF analyzer [45]. MALDI TOF-TOF, MALDI ion source has been coupled to two types of TOF instruments. In the first, second TOF sections are separated by a collision cell [46]. These mass spectrometers have 
high sensitivity, resolution and mass accuracy, and can be widely used in proteomic research.

\subsubsection{Tandem Mass Spectrometry}

Tandem mass spectrometry (MS/MS), as the name implies, involves two stages of MS. In the first stage, ions of a desired $\mathrm{m} / \mathrm{z}$ are isolated (precursor ions). The isolated ions are increased the internal energy, and are induced to collide with an inert gas such as helium, argon or nitrogen, leading to dissociation. The resulting ions (product ions) are analyzed with the second stage of MS. MS/MS is a key technique for protein or peptide sequencing and PTMs analysis. Collision-induced dissociation (CID) has been the most widely used MS/MS technique in proteomics research. In this method, a particular gas phase peptide/protein ions is isolated and subsequently the energy is imparted by collisions with inert gas. The energy causes the peptide to break apart on the peptide backbone. Figure 1.6 shows how the peptide fragment and how the fragment ions are designated.

(A)

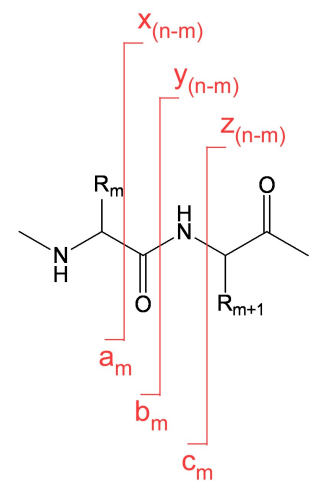

(B)

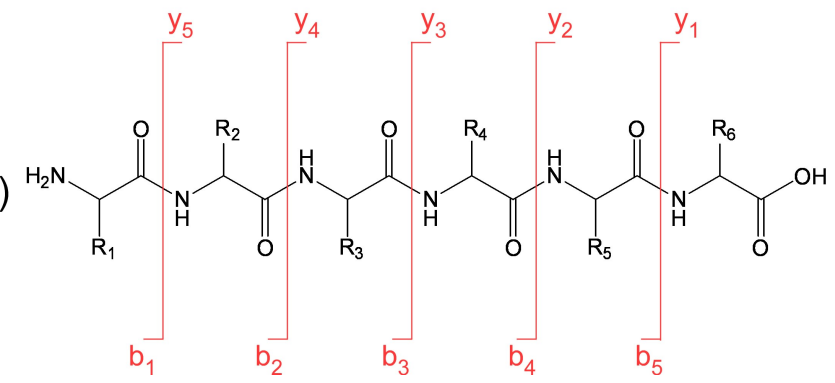

Figure 1.6. The types of peptide fragment ions observed in a MS/MS spectrum. (A) When the charge is localized on the $\mathrm{N}$-terminus, the ion is classed as $a_{m}, b_{m}$ or $c_{m}$. When the charge is localized on the C-terminus, the type of ion is classed as $x_{(n-m)}$, $y_{(n-m)}$ or $z_{(n-m)}$. (B) The fragment of peptide is induced by collisions with inert gas, and the bond breakage mainly occurs in the lowest energy pathway. That is cleavage of the amide bonds, which leads to form $b$ - and $y$-ions series.

The most common and informative ions are generated by fragmentation at amide bond between amino acids. The resulting ions are called b-ions if the charge is localized on the $\mathrm{N}$-terminal part of the peptide and $\mathrm{y}$-ions if the charge is localized on the C-terminal part [47, 48]. The common proteomic experiment is performed with trypsin digestion .The resulting peptides have arginyl or lysyl residues at their C-terminus. In this case, the $y$-ion series are the predominant in the spectra. For an even more in-depth characterization, the fragment ions of peptide can be further fragmented. This is known as $\mathrm{MS}^{3}$ or more generally, $\mathrm{MS}^{\mathrm{n}}$. It is worth to 
note that the internal fragmentation and neutral losses of $\mathrm{H}_{2} \mathrm{O}, \mathrm{NH}_{3}$, and labile PTMs occur frequently during the CID process.

\subsection{Data Analysis}

As mentioned before, MALDI-TOF MS is still much used to identify protein by what is known as peptide mapping, also referred to as peptide mass fingerprinting (PMF), due to its simplicity, excellent mass accuracy, high resolution and sensitivity. In this method, protein is identified by matching a list of experimental peptide masses with the calculated list of all peptide masses of each entry in a protein database (SwissProt, NCBInr and etc.). Due to the fact that mass mapping requires an essentially purified target protein, the technique is commonly used in combination with protein separation technology using either SDS-PAGE or 2DE, respectively. However, protein identification based solely on PMF should no longer be acceptable and must be complemented by MS/MS spectra. Because the MS/MS spectra contain structural information related to the sequence of the peptide, in addition to precursor ion mass information, these searches are generally more specific and discriminating. Several MS/MS spectra searching engines exist such as Sequest [49] and MASCOT [50]. The MS/MS spectra are collected as many as possible, and the results are searched by an algorithmic comparison via Sequest or MASCOT towards a protein database. These methods do not attempt to extract any sequence information at all from the MS/MS spectrum. Instead, the experimental fragment spectrum is matched against a calculated fragment spectrum for all peptides in the database. A score is given to determine how close between MS/MS spectrum and the calculated peptide sequence. Sequest, a cross-correlation method, peptide sequences in the database are used to construct theoretical MS/MS spectra. The overlap or cross-correlation of these predicted MS/MS spectra with the experimental MS/MS spectra determines the best match. MASCOT, a probability based matching, the calculated fragment ion from peptide sequences in the database are compared with experimental MS/MS spectra. From this comparison, a score is calculated which reflects the statistical significance of the match between the MS/MS spectrum and the sequence contained in the database. In each of these methods, the identified peptides are compiled into a protein hit list, which is the output of a typical proteomic experiment. The protein identification relies on the matches with sequence database, and not all peptides resulting from the enzymatic digestion of a protein can be observed or correctly identified with MS analysis. This would reflect especially on the peptide with unexpected PTMs, and high-throughput proteomics is currently limited largely due to those species for which comprehensive sequence databases are available.

In brief, proteomics is the large-scale study of proteins, particularly their structures and functions. MS-based proteomics has become a formidable tool for the identification of proteins. The limitation in dynamic range of MS analysis only allows for the proteins present at high 
relative abundance to be preferentially identified, while information regarding the proteins present at low abundance in the complex mixtures is commonly not detected. Hence, the development of separation technology and the continued improvement of mass spectrometric methodology is crucial for identifying the low abundance proteins. 
Chapter 2 - A High-Throughput Method for Phosphopeptide Enrichment of Spliceosomal Proteins and Its Applications

\subsection{Summary}

Reversible protein phosphorylation is a ubiquitous post-translational modification critical to many cellular processes. In this study, a simple, inexpensive and convenient titanium dioxide $\left(\mathrm{TiO}_{2}\right)$ microspin column fabricated in a commercial pipette tip was developed for high-throughput enrichment of phosphopeptides in a crude mixture of spliceosomal proteins, which were digested by trypsin. The spliceosome is a protein-RNA complex which catalyses the excision of introns and ligation of exons of eukarytic pre-mRNAs. Our approach allows the enrichment of twenty-four samples at once. Careful comparison of our novel high-throughput method with the previously described manual $\mathrm{TiO}_{2}$ enrichment techniques showed similar result in terms of selectivity and sensitivity. Additionally, we evaluated and optimized the titania-based affinity enrichment for global profiling of phosphopeptides in total small nuclear ribonucleoproteins (snRNPs). We found that the use of RapiGest ${ }^{\mathrm{TM}} \mathrm{SF}$ as detergent during digestion was more efficient than urea. The non-specific binding of non-phosphorylated peptides on $\mathrm{TiO}_{2}$ materials was reduced, but still maintained the high binding affinity of phosphopeptides without the need for an additional desalting step. Approximately $70 \%$ of the enriched peptides were identified by mass spectrometry as being phosphorylated. Furthermore, a complementary integrated analytical platform involving a combination of in-solution digestion, in-gel digestion from SDS-PAGE, $\mathrm{TiO}_{2}$ microspin columns, on-line nanoLC ESI-MS and off-line nanoLC MALDI-MS was employed to discover the maximum number of phosphorylation sites in the human spliceosomal proteins present in the crude mixture of nuclear snRNP particles. These strategies allow the complementary measurement of phosphopeptides. When compared with off-line nanoLC MALDI-MS/MS, online LC-ESI MS/MS turned out to be better for determining the exact location of the phosphorylation site. In total, 1381 phosphopeptides were identified in 390 proteins; of these, 640 sites were not previously described. The list of phosphopeptides was used to extract known and novel kinase motifs using the Motif-X algorithm. Finally, we showed three applications of this methodology for identifying phosphorylation sites and for studying protein-RNA crosslinks.

\subsection{Introduction}

A spliceosome is a complex of specialized ribonucleic acid (RNA) and protein subunits that removes introns from a transcribed precursor messenger ribonucleic acid (pre-mRNA) segment. The process is generally referred as splicing. Each spliceosome is composed of small nuclear RNA proteins, called snRNPs, and a range of non-snRNP associated protein factors. The snRNPs that make up the nuclear spliceosome are named U1, U2, U4, U5, and 
U6, and participate in several RNA-RNA and RNA-protein interactions. The snRNPs display a broad variety of molecular sizes and chemical properties such as Arg-/Ser-rich tracts. They are ideally suited to establish methods for analysis of the changes in the phosphorylation during splicing process. Human spliceosome assembly intermediates have been observed include the $E, A, B, B^{*}$, and $C$ complex shown in Figure 2.1 [51]. The first recognition of pre-mRNA involves U1 snRNP binding to the $5^{\prime}$ end splice site of the pre-mRNA and other non-snRNP associated factors to form the E complex. Subsequently, the U2 snRNP tightly associates with the branch point sequence (BPS) with the E complex to form A complex. The U4/U6.U5 tri-snRNP stably interacts to the assembling spliceosome to form complex B. Following several rearrangements in RNA-RNA and RNA-protein interactions, detaching the U1 and U4 snRNPs, give rise to the catalytically activated spliceosome $\left(B^{*}\right.$ complex $)$ and then converts into $C$ complex, in which the first of the two catalytic steps of splicing has occurred. After the second step, the spliceosome dissociates and the snRNPs are recycled for repeated rounds of spliceosome assembly.

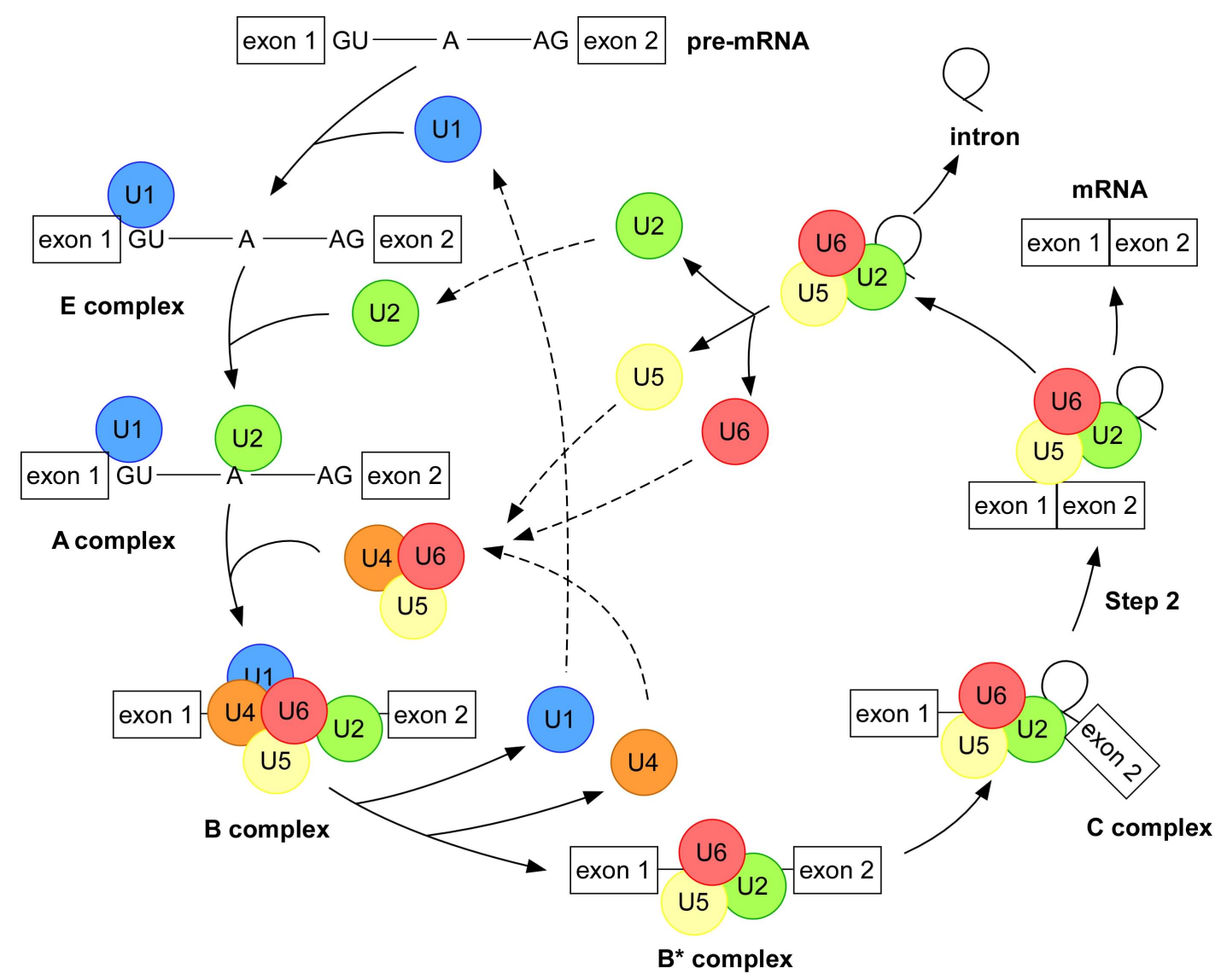

Figure 2.1. The pathway of spliceosome assembly.

PTMs of spliceosomal proteins play a crucial role in triggering conformational changes in protein-protein and RNA-protein interactions during the spliceosome assembly that are 
essential for its activity. For example, pre-mRNA splicing can be regulated both positively and negatively by reversible protein phosphorylation [52]. The phosphorylation of SR proteins are mediated by protein kinases / phosphatases and have been shown to be required for the formation of a catalytically active spliceosome [53-55]. Hence, an important focus of future will be to identify PTMs in spliceosomal proteins, and determine whether their modifications regulated during the splicing process.

Protein phosphorylation is one of the most common post-translational modifications and plays an important role in the regulation of a variety of cellular events, including signaling, cell differentiation, metabolism and apoptosis [56, 57]. Hence, the characterization of phosphorylation is a key issue in current proteomic research. Recently, MS-based techniques have been widely applied as powerful tools to characterize protein modifications, including phosphorylation, due to its speed, reliability, high sensitivity and capability for determining phosphorylation sites by MS/MS sequencing. However, large-scale phosphoproteomic analysis still remains a substantial challenge due to low abundance of phosphopeptides combined with large amounts of non-phosphorylated peptides which tend to suppress the ion signal of phosphorylated peptides in MS analysis [58-61]. Therefore, the highly specific separation and enrichment of phosphopeptides from proteolytic digest mixture becomes a critical step prior to MS analysis.

An effective method to resolving these problems is selective enrichment of phosphorylated peptides before MS analysis, like strong cation exchange (SCX) chromatography [62-66], strong anion exchange (SAX) chromatography [62, 64], immobilized metal ion affinity chromatography (IMAC) [67-74] and metal oxide affinity chromatography (MOAC) [75-88]. Among them, IMAC is commonly used and successfully coupled to various mass spectrometries. With this approach the phosphopeptides are captured by chelating interaction with metal ion such as $\mathrm{Fe}^{3+}$ or $\mathrm{Ga}^{3+}$ shown in Figure 2.2.

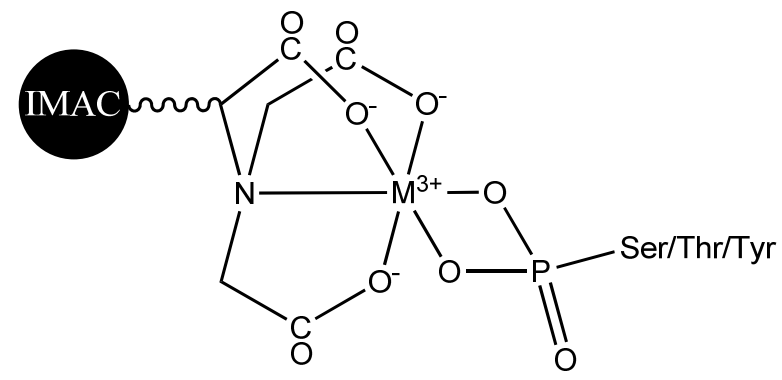

Figure 2.2. Phosphopeptide enrichment with immobilized metal ion affinity chromatography (IMAC). The binding between a phosphopeptide and IMAC resin is shown. The IMAC stationary phase is made by chelation of iminodiacetic acid (IDA) or nitrilotriacetic acid (NTA) with triply charged iron $\left(\mathrm{Fe}^{3+}\right)$ or gallium $\left(\mathrm{Ga}^{3+}\right)$ ions. 
Enrichment and recovery strongly depend on the type of metal ion, column material, and loading/eluting procedures that are used. However, non-specific enrichment is unavoidable. Acidic peptides tend to bind to the metal resins which complicate the MS detection of enriched phosphopeptides. Such non-specific binding can be reduced by prior methyl esterification of the acidic side chains of amino acid residues [89-92]. Nevertheless, incompleteness and side reactions of methyl esterification process can increase complexity of MS analysis and data interpretation, leading to decrease sensitivity.

Alternative to IMAC, more recently metal oxide affinity chromatography (MOAC) has been demonstrated to be effective material for the selective enrichment of phosphopeptides from proteolytic digests based on chelating interaction between phosphate functional groups and the surface of metal oxide particles. Metal oxides such as $\mathrm{TiO}_{2}, \mathrm{ZrO}_{2}, \mathrm{Fe}_{3} \mathrm{O}_{4}$ and $\mathrm{Al}_{2} \mathrm{O}_{3}$ are due to their high chemical stability ) [75-88]., tolerance over a broader $\mathrm{pH}$ range and physical robustness; hence, some non-volatile acidic additives, including 2,5-dihydroxybenzoic acid (DHB), phthalic acid (PA) and acidic buffers, can be employed to avoid non-specific binding $[75,88]$. Among them, $\mathrm{TiO}_{2}$ has been widely used to selectively capture phosphopeptides shown in Figure 2.3.

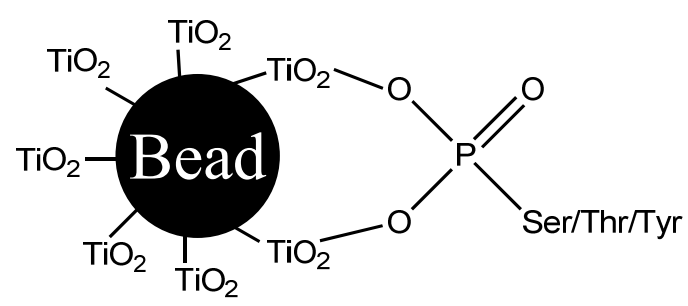

Figure 2.3. Phosphopeptide enrichment with titanium dioxide $\left(\mathrm{TiO}_{2}\right)$ bead. The binding between a phosphopeptide and $\mathrm{TiO}_{2}$ coated resin is shown

Larsen et al. demonstrated that an addition of high quantity DHB in the loading and washing buffer could reduce non-specific bindings on $\mathrm{TiO}_{2}$ column [75]. However, although a diversity of techniques is available for phosphopeptides enrichment, the mapping of entire phosphoproteome is still a challenging task.

In this study, we invented a simple-to-fabricate, easy-to-use, economic and high efficiency $\mathrm{TiO}_{2}$ microspin column to enrich phosphopeptides. This design reduces the entire analyzed time in large scare analysis. Three commercially available articles for daily use - coffee filter, pipette tip and eppendorf tube - are utilized to fabricate microspin column. In addition, we integrated our microspin column with different proteomic technique to explore the phosphorylation sites in a crude mixture of nuclear snRNP particles, individual U snRNP, spliceosomal complexes and SR proteins. 


\subsection{Experiment Sections}

\subsubsection{Materials}

lodoacetamide (IAA), $\alpha$-cyano-4-hydroxy-cinnamic acid (CHCA), 2,5-dihydroxybenzoic acid (DHB), phthalic acid (PA), trifluoroacetic acid (TFA), [Glu]-Fibrinopeptide B (Glu-Fib) and ammonium bicarbonate were obtained from Sigma-Aldrich (St. Louis, MO). Sequencing grade, modified trypsin was obtained from Promega (Madison, WI). Dithiothreitol (DTT), formic acid, ammonia solution, acetonitrile (ACN), and ethanol were obtained from Merck (Darmstadt, Germany). RapiGest ${ }^{\mathrm{TM}}$ SF was obtained from Waters Corporation (Manchester, UK). Titanium dioxide $\left(\mathrm{TiO}_{2}\right)$ resins were obtained from GL Sciences Inc. (Tokyo, Japan).

\subsubsection{A Crude Mixture of Nuclear snRNP Particles, Individual U snRNP, Spliceosomal Complexes and SR Proteins Purification}

U1, U2, U5 snRNPs and U4/U6.U5 tri-snRNPs were isolated from HeLa nuclear extract by anti-m3G cap-directed immunoaffinity purification with the m3G-specific antibody $\mathrm{H}-20$ to obtain a crude mixture of nuclear snRNP particles [93] and followed glycerol gradient centrifugation to separate the individual snRNPs as described previously [94, 95]. Spliceosomal A, B and C complexes were isolated from in vitro splicing reactions by the MS2 affinity-selection method $[96,97]$. SR proteins were isolated by two salt precipitation steps, ammonium sulfate and magnesium chloride precipitation [98]. All protein complexes were purified by Prof. Reinhard Lührmann's Laboratory.

\subsubsection{Ethanol Precipitation}

The purified protein complexes were precipitated by adding 3 volumes of ethanol and 1/10 volume of $3 \mathrm{M}$ sodium acetate, $\mathrm{pH}$ 5.3. The mixture was vortexed, incubated at $-20{ }^{\circ} \mathrm{C}$ for 2 hours and then centrifuged $17000 \mathrm{~g}$ at $4{ }^{\circ} \mathrm{C}$ for $30 \mathrm{~min}$. The supernatant was removed and the pellet was washed with $500 \mu \mathrm{l}$ of $80 \%$ ethanol and centrifuged as above. Discarded supernatant, the pellet was evaporated with a SpeedVac.

\subsubsection{In-Solution Digestion}

Precipitated proteins were dissolved with $20 \mu \mathrm{l}$ of $1 \%$ RapiGest $^{\mathrm{TM}} \mathrm{SF}$ in $25 \mathrm{mM}$ ammonium bicarbonate, $\mathrm{pH} 8.5$, sonicated for $15 \mathrm{~min}$, reduced with $10 \mu \mathrm{l}$ of $50 \mathrm{mM}$ DTT at $37{ }^{\circ} \mathrm{C}$ for 1 hour, alkylated with $10 \mu \mathrm{l}$ of $100 \mathrm{mM}$ IAA at $37{ }^{\circ} \mathrm{C}$ for 1 hour, diluted with $60 \mu \mathrm{l}$ of $25 \mathrm{mM}$ ammonium bicarbonate, and subsequently digested with trypsin (1:20 enzyme to substrate ratio) at $37{ }^{\circ} \mathrm{C}$, overnight. Tryptic peptides were acidified with $50 \mu \mathrm{l}$ of $5 \%$ TFA at $37{ }^{\circ} \mathrm{C}$ for 2 hours and followed centrifugation at $17000 \mathrm{~g}$ for $10 \mathrm{~min}$. The resulting supernatant was transferred to another eppendorf and dried on a SpeedVac for further analysis. Another 
procedure employed urea as a denaturation reagent instead of RapiGest ${ }^{\mathrm{TM}} \mathrm{SF}$. The ethanol precipitated proteins were dissolved with $20 \mu \mathrm{l}$ of $6 \mathrm{M}$ urea, sonicated, reduced, alkylated and digested as above and subsequently evaporated with a SpeedVac.

\subsubsection{In-Gel Digestion}

Precipitated proteins were separated by electrophoresis on a 4-12 \% NuPAGE $®$ Novex Bis-Tris Gels system (Invitrogen Corporation, Carlsbad, CA). The gel was stained with Coomassie Blue and cut equally into twenty slices. Each slice was reduced with $60 \mu \mathrm{l}$ of 50 mM DTT at $37{ }^{\circ} \mathrm{C}$ for 1 hour, alkylated with $60 \mu \mathrm{l}$ of $100 \mathrm{mM} \mathrm{IAA}$ at $37{ }^{\circ} \mathrm{C}$ for 1 hour, and subsequently digested with trypsin at $37{ }^{\circ} \mathrm{C}$, overnight (the enzyme to substrate ratio is $1: 20$ ) as described previously [99].

\subsubsection{In-House $\mathrm{TiO}_{2}$ Microspin Column Fabrication}

A small plug of coffee filter $(1 \mathrm{~mm} \times 1.5 \mathrm{~mm})$ was placed at the end of the tip by using a capillary tube shown in Figure. 2.4. The coffee filter serves only as a frit to retain the $\mathrm{TiO}_{2}$ resins within the commercial pipette tip. A length of approximately $3 \mathrm{~mm}$ of $\mathrm{TiO}_{2}$ materials was packed in the end of a pipette tip. Subsequently, the pipette tip was place into an open hole eppendorf for phosphopeptide enrichment.

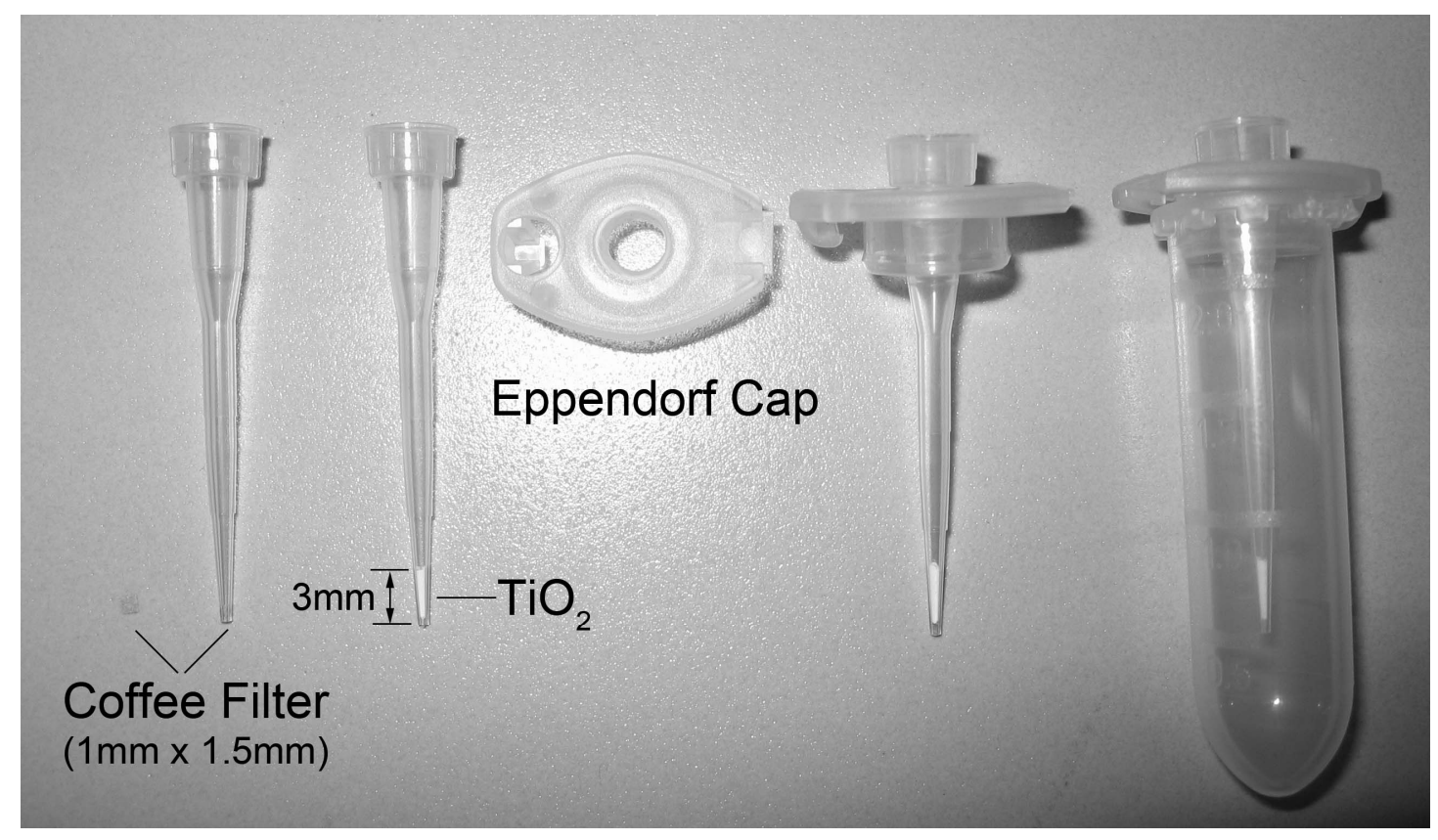

Figure 2.4. Picture of a self-made $\mathrm{TiO}_{2}$ microspin column.

\subsubsection{Comparison of Different $\mathrm{TiO}_{2}$ Enrichment Procedure}


Six different procedures for phosphopeptide enrichment were investigated shown in Figure 2.5. In procedure A, tryptic peptides were dissolved with $60 \mu \mathrm{l}$ of $200 \mathrm{mg}$ 2,5-dihydroxybenzoic acid $(\mathrm{DHB})$ in $1 \mathrm{ml}$ of $80 \% \mathrm{ACN}, 5 \% \mathrm{TFA}$ and then loaded onto $\mathrm{TiO}_{2}$ microspin column. The column was washed three times with $60 \mu \mathrm{l}$ of $200 \mathrm{mg}$ DHB in $1 \mathrm{ml}$ of $80 \% \mathrm{ACN}, 5 \%$ TFA and five times with $60 \mu \mathrm{l}$ of $80 \%$ ACN, $5 \%$ TFA. Bound peptides were eluted three times with $40 \mu \mathrm{l}$ of $0.3 \mathrm{~N} \mathrm{NH}_{4} \mathrm{OH}(\mathrm{pH} \geq 10.5)$, and subsequently evaporated $\mathrm{NH}_{4} \mathrm{OH}$ with a SpeedVac for further analysis. The speed of centrifugation for each step had to be controlled at $3000 \mathrm{rpm}$ for $5 \mathrm{~min}$. Procedure $\mathrm{B}$ and $\mathrm{C}$ were similar to the procedure $\mathrm{A}$ with the exception that an additional desalted step with $\mathrm{C} 18$ (Nucleosil 100-5 C18) or $\mathrm{NH}_{2}$ (Nucleosil 100-5 $\mathrm{NH}_{2}$ ) microspin column was employed before $\mathrm{TiO}_{2}$ enrichment, respectively. Procedure $\mathrm{D}, \mathrm{E}$ and $\mathrm{F}$ were similar to procedure $\mathrm{A}, \mathrm{B}$ and $\mathrm{C}$, respectively, with the exception that the loading and washing buffer were replaced with saturated phthalic acid (PA) in $80 \%$ ACN, $5 \%$ TFA.

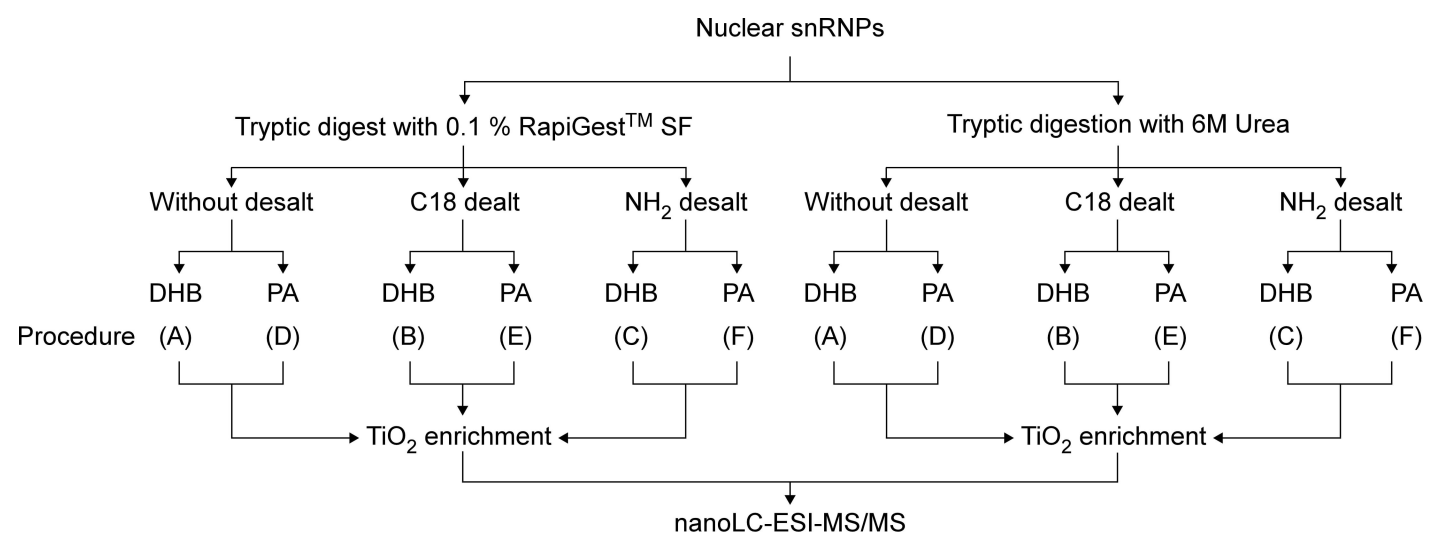

Figure 2.5. Optimization of the purification procedure using microspin $\mathrm{TiO}_{2}$ column.

\subsubsection{NanoLC-ESI and -MALDI Mass Spectrometry Analysis.}

The resulting peptides were first loaded at a flow rate of $10 \mu \mathrm{l} / \mathrm{min}$ onto an in-house packed C18 trap column $(1.5 \mathrm{~cm}, 360 \mu \mathrm{m}$ o.d., $150 \mu \mathrm{m}$ i.d., Nucleosil 100-5 C18, Macherey-Nagel, $\mathrm{GmbH} \& \mathrm{Co} . \mathrm{KG})$. The retained peptides were then eluted and separated on an analytical $\mathrm{C} 18$ capillary column (30 cm, $360 \mu$ m o.d., $75 \mu \mathrm{m}$ i.d., Nucleosil 100-5 C18) at a flow rate of 300 $\mathrm{nL} / \mathrm{min}$, with a gradient from 7.5 to $37.5 \% \mathrm{ACN}$ in $0.1 \%$ formic acid for $60 \mathrm{~min}, 120 \mathrm{~min}$ or 240 min, using an Agilent 1100 nano-flow LC system (Agilent Technologies, Palo Alto, CA), coupling with Z-spray source or a robotic Probot $\mathrm{TM}$ micro fraction collector (LCPackings/Dionex, Sunnyvale, CA) for ESI-MS and -MS/MS (Waters/Micromass Q-Tof Ultima TM API mass spectrometer, Milford, MA) or MALDI-MS and -MS/MS (4800 MALDI-ToF/ToF, Applied Biosystems, Framingham, MA) analysis, respectively. For ESI-MS and -MS/MS data dependent acquisition, $1 \mathrm{~s}$ survey scans were run over the mass range $\mathrm{m} / \mathrm{z}$ 350 to 1600 . A maximum of three concurrent MS/MS acquisitions were triggered for $2+, 3+$, 
and $4+$ charged precursors detected at intensity above 15 counts, after 3 s of acquisition, the system switched back to survey scan mode. For LC-MALDI analysis, The eluent was channelled to a nano-Tee where it was premixed with a matrix solution $(10 \mathrm{mg} / \mathrm{mL}$ $\alpha$-cyano-4-hydroxy-cinnamic acid, CHCA and $2.5 \mathrm{fmol} / \mu \mathrm{l} \mathrm{Glu}-\mathrm{Fib}$ in $80 \% \mathrm{ACN}, 0.1 \% \mathrm{FA}$ ) delivered at $1.7 \mu \mathrm{l} / \mathrm{min}$ by a syringe pump and directly spotted onto 384-well MALDI sample plate in every $30 \mathrm{~s}$. Subsequently, MALDI-MS and -MS/MS detected and sequenced peptides. 1000 and 5000 shots were accumulated in positive ion mode MS and MS/MS, respectively. For collision-induced dissociation (CID) MS/MS operation, argon was used as collision gas and the indicated cell pressure was set up $5 \times 10^{-7}$, with the potential difference between the source acceleration voltage and the collision cell set at $1 \mathrm{kV}$. The resolution of time ion selector for precursor ion was set at 200. MS spectra were acquired using Glu-Fib for internal calibration and MS/MS spectra were acquired using instrument default calibration.

\subsubsection{Interpretation of Tandem Mass Spectra}

All spectra were searched MASCOT server v2.2.0.6 against the NCBInr database limited to human with criteria-peptide mass tolerance, $50 \mathrm{ppm}$; MS/MS ion mass tolerance, $0.25 \mathrm{Da}$; allowed up to three missed cleavage; variable modifications were considered phosphorylation of serine, threonine and tyrosine, methionine oxidation and cysteine carboxyamidomethylation. The significant protein hits defined as protein score must be higher than 50 and if the protein score was between 20 and 50, we manually evaluated each MS/MS spectra. The threshold for individual peptide score must be higher than 20 and required bold red. All phosphorylated sited were examined manually by the presence of a $69 \mathrm{Da}$ between fragment ions for phosphoserine and an $83 \mathrm{Da}$ for phosphothreonine. Motif analysis was performed using motif-x [100] (http://motif-x.med.harvard.edu/) with significance set to 0.000001 and occurrence set to 20 , using the human IPI as background. All amino acid frequency plots weblogos [101] (http://weblogo.berkeley.edu/) were created as frequency plots.

\subsection{Result and Discussion}

\subsection{1 $\mathrm{TiO}_{2}$ Microspin Column}

Recent reports have shown that $\mathrm{TiO}_{2}$ can be widely used to enrich phosphopeptides from peptide mixtures; however, the entire enriched procedure requires approximately $15 \mathrm{~min}$ for each sample $[75,102,103]$. Hence, we set out to establish a robust and fast (i.e. semi-high-throughput) enrichment procedure for phosphopeptides derived from in-gel digestion and in-solution digestion. $\mathrm{TiO}_{2}$ materials are packed into a pipette tip that contains ordinary (coffee) filters as a frit to prevent leakage of $\mathrm{TiO}_{2}$ resins during the enrichment procedure (Figure 2.4). Hundreds of $\mathrm{TiO}_{2}$ microspin column were fabricated and tested during the period of this study, but we did not find any $\mathrm{TiO}_{2}$ resins slip out of the column. The system 
has the further advantage that the device is small enough to fit into a 1.5 or $2 \mathrm{ml}$ eppendorf tube. It can thus be easily placed into a benchtop laboratory centrifuge with a rotor for $1.5(2 \mathrm{ml})$ eppendorf tubes. In this manner 24 samples (using a standard rotor) can be processed in parallel, the cost is reasonable and one-time usage eliminates the risk of carryover. In contrast, phosphopeptide enrichment with $\mathrm{TiO}_{2}$ microcolumns packed with gel loader tips, which are widely used in many labs, is much more time consuming due to manual handling, i.e. loading, washing and eluting procedure.

\subsubsection{Sensitivity of the $\mathrm{TiO}_{2}$ Microspin Column}

In initial experiments the specificity and sensitivity of our semi-automatic device for the enrichment of phosphopeptides was tested and compared with that of $\mathrm{TiO}_{2}$ microcolumn packed in gel loader tip [75]. We examined the power of $\mathrm{TiO}_{2}$ enrichment for phosphopeptides that derived from the arginine/serine-rich splicing factor SFRS1, SC35 and 9G8 separated by SDS-PAGE [98] as showed in Figure 2.6.

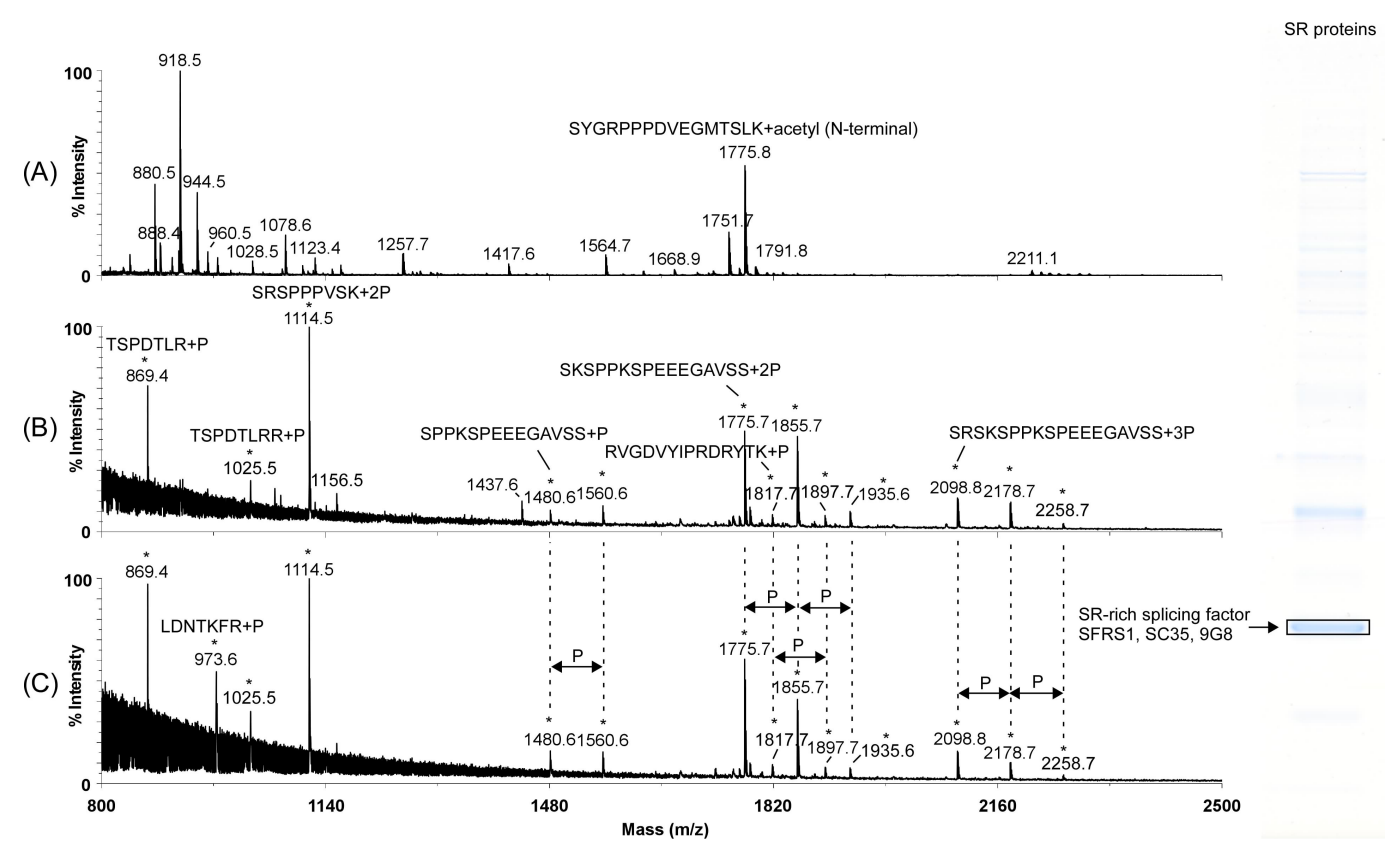

Figure 2.6. MALDI mass spectra of tryptic peptides of arginine/serine-rich splicing factor SFRS1, SC35, 9G8. (A) Before enrichment. (B) Enrichment with the existing manual $\mathrm{TiO}_{2}$ microcolumn. (C) Enrichment with our $\mathrm{TiO}_{2}$ microspin column. The inset shows the SDS-PAGE gel image from purified SR proteins. The signals of phosphorylated peptides are marked with asterisk *.

Fig 2.6A showed a direct MALDI peptide mass fingerprint of an aliquot of extracted tryptic peptides derived from arginine/serine-rich splicing factor SFRS1, SC35 and 9 G8 without enrichment. The resulting spectrum was dominated by the signals of non-phosphorylated 
peptides. After enrichment with $\mathrm{TiO}_{2}$ microcolumn packed in gel loader tip (Figure 2.6B) or with our $\mathrm{TiO}_{2}$ microspin column (Figure 2.6C, see Experiment Section 2.3.7, Procedure A in detail), the resulting spectra indicated the majority of non-phosphorylated peptides were removed, and the signals corresponding to the phosphopeptides were enhanced (marked with a star), which were confirmed by ESI-MS/MS (the peptide sequence with the phosphorylation site is listed within the spectrum, see also Appendix 1). In total, fourteen phosphopeptide signals were observed in both enrichment methods. One additional phosphopeptide signal at $\mathrm{m} / \mathrm{z}$ 973.6 was found during enrichment with our $\mathrm{TiO}_{2}$ microspin column identified by our microspin column.

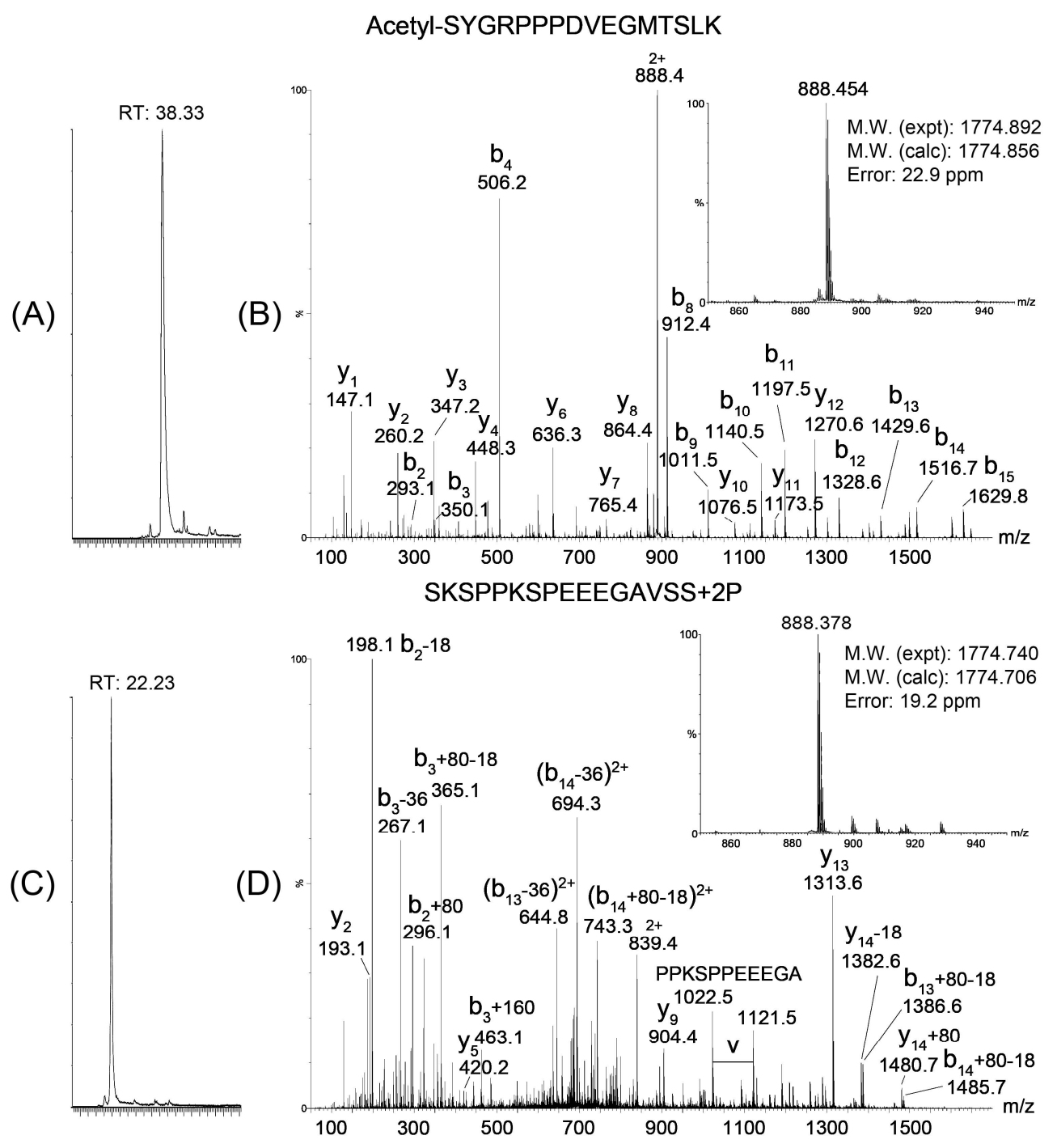

Figure 2.7. (A-B) Extracted ion chromatograms of peptide acetyl-SYGRPPPDVEGMTSLK at $\mathrm{m} / \mathrm{z} 888.454$ and phosphopeptide SKSPPKSPEEEGAVSS at $\mathrm{m} / \mathrm{z} 888.378$, respectively. (C-D) MS/MS spectra of peptide acetyl-SYGRPPPDVEGMTSLK and phosphopeptide SKSPPKSPEEEGAVSS, respectively. The inset showed the $\mathrm{m} / \mathrm{z}$ of precursor ion in the mass spectrum. 
Strikingly, the abundant peak in the non-enriched spectrum at $\mathrm{m} / \mathrm{z} 1775.8$ (Figure 2.6A) was shown by MS/MS spectrum to be the N-terminal acetylated peptide SYGRPPPDVEGMTSLK of $9 \mathrm{G} 8$ protein (Figure 2.7B), whereas in both the enriched samples at $\mathrm{m} / \mathrm{z} 1775.7$ (Figure 2.6B and 2.6C), it was found to be the phosphopeptide SKSPPKSPEEEGAVSS (Figure 2.7D). The mass difference between these two peaks was $0.152 \mathrm{Da}$. They were eluted separately from LC column at a retention time of $38.33 \mathrm{~min}$ and $22.23 \mathrm{~min}$, respectively (Figure 2.7A and 2.7C). The specificity and sensitivity of our $\mathrm{TiO}_{2}$ microspin column is equivalent to existing manual microcolumn. Importantly, our $\mathrm{TiO}_{2}$ microspin column allows a significant higher sample throughput within a given time. In this manner phosphopeptides derived from proteins from in an entire gel lane that was cut into 23-24 slices (e.g. NUPAGE gels) can be enriched in parallel (see also below).

\subsubsection{Optimizing Phosphopeptide Enrichment with $\mathrm{TiO}_{2}$ microspin column for In-Solution Digestion}

We next made use of the high-throughput capabilities of the $\mathrm{TiO}_{2}$ microspin columns and systematically tested for maximum selectivity and sensitivity upon various in-solution digestion procedure, desalting steps and washing conditions that were most favorable to identify the maximum number of phosphopeptides. A crude mixture of total $U$ snRNPs isolated from Hela nuclear extraction by immunoaffinity chromatography [93] was used as a test system.

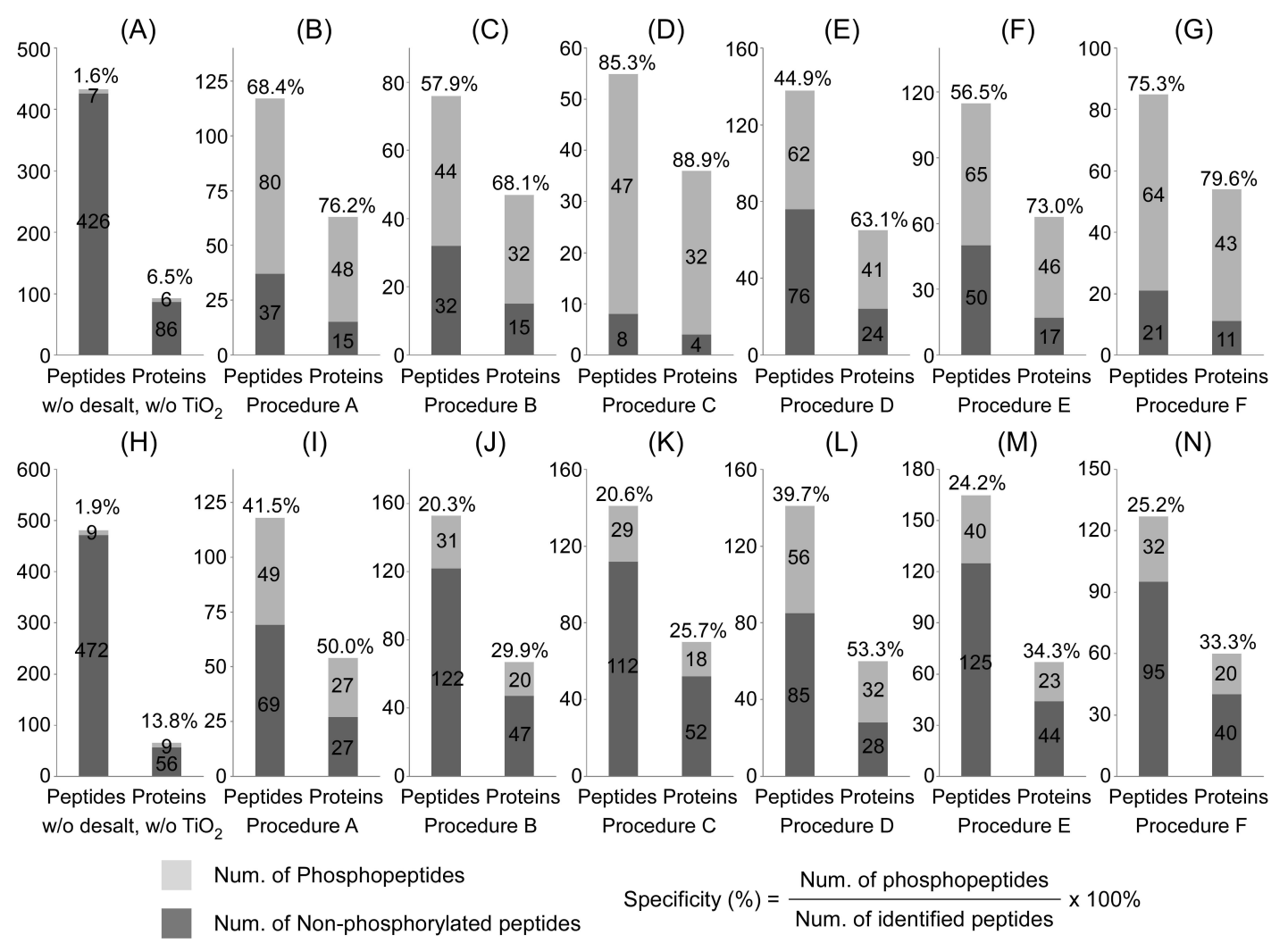


Figure 2.8. Selectivity and sensitivity of the different phosphopeptides enrichment strategies using $\mathrm{TiO}_{2}$ microspin column. Various surfactants, desalting steps, loading and washing buffers were compared to identify the maximum number of phosphopeptides and phosphoproteins by nanoLC-ESI-MS/MS as shown in A-N, respectively. (A-G) $10 \mu \mathrm{g}$ total $U$ snRNPs were digested in the presence of RapiGest ${ }^{T M}$ SF. (H-N) instead of RapiGest ${ }^{\mathrm{TM}}$ SF with urea. $(\mathrm{A})$ and $(\mathrm{H})$ without enrichment. $(\mathrm{B})$ and $(\mathrm{I})$, procedure $A$, tryptic peptides were dissolved with 2,5-dihydroxybenzoic acid (DHB) buffer and subsequently loaded onto $\mathrm{TiO}_{2}$ microspin column; washed with DHB buffer and $5 \%$ TFA in $80 \%$ ACN and then eluted with $\mathrm{NH}_{4} \mathrm{OH}$. (C) and $(\mathrm{J})$, procedure $\mathrm{B}$, an additional $\mathrm{C} 18$ desalting step was performed prior to $\mathrm{TiO}_{2}$ enrichment. (D) and $(\mathrm{K})$, procedure $\mathrm{C}$, instead of $\mathrm{C} 18$ with $\mathrm{NH}_{2}$ material. $(E)$ and $(L)$, procedure $D,(F)$ and $(M)$, procedure $E,(G)$ and $(M)$, procedure $F$, were the same as procedure $A, B$ and $C$, respectively, with the exception that loading and washing buffer were replaced with saturated phthalic acid (PA). (see Experiment Section 2.3.7 and Figure 2.5 in detail). Specificity: Num. of phosphopeptides/Num. of identified peptides $\times 100 \%$.

Initial optimization of the procedure was evaluated by testing first different surfactant on the impact of $\mathrm{TiO}_{2}$ enrichment procedure. We first compared urea and RapiGest ${ }^{\mathrm{TM}} \mathrm{SF}$ as denaturing agent (Figure 2.8.A-G and 2.8H-N, respectively). $10 \mu \mathrm{g}$ of a crude mixture of total $\mathrm{U}$ snRNPs was denatured, reduced, alkylated and digested with trypsin over night. The resulting peptides were enriched as above (see Experiment Section 2.3.7 and Figure 2.5 in detail) and eluted peptides were analyzed by LC-ESI-MS/MS. Without enrichment only seven and nine phosphopeptides were identified in RapiGest ${ }^{\mathrm{TM}} \mathrm{SF}$ and urea denatured sample, respectively (Figure $2.8 \mathrm{~A}$ and $2.8 \mathrm{H}$ ). In general, the total number of peptides derived from urea treated samples was higher as compared to RapiGest ${ }^{\mathrm{TM}} \mathrm{SF}$ as the total number of peptides included those that are carbamylated at $\mathrm{N}$-terminus, lysine, arginine and cysteine residues as shown in Figure 2.9 [104-106].

We found that digestion with RapiGest ${ }^{\mathrm{M}} \mathrm{S}$ SF significantly increased the number of sequenced phosphopeptides (Figure 2.8B-G compared to 2.8I-N) and reduced the number of less non-phosphorylated peptides as compared to urea treatment. This can be explained several reasons: First, urea decomposes into ammonium cation and ammonium cyanate during the digestion process (Figure 2.9A). The ammonium cation elutes phosphopeptides from $\mathrm{TiO}_{2}$ resins and therefore decreases the number of phosphopeptides that can be identified. Secondly, the lone pair electrons of nitrogen on the carbamyl group of carbamaylated peptides also binds to $\mathrm{TiO}_{2}$ resins. Third, RapiGest ${ }^{\mathrm{TM}} \mathrm{SF}$ is an acid-labile surfactant. It hydrolyzes in acid solution posterior to in-solution enzymatic digestion to form sulfonic sodium salt in the sample shown in Figure 2.10 [107]. Carboxyl acid peptides in the sample that also bound to 
$\mathrm{TiO}_{2}$ beads are displaced by the sulfonic anion but phosphopeptides were still retained, therefore, the specific for phosphopeptide in $\mathrm{TiO}_{2}$ enrichment is increased when using RapiGest $^{\mathrm{TM}}$ SF $[84,102]$.

(A) Decomposition of urea

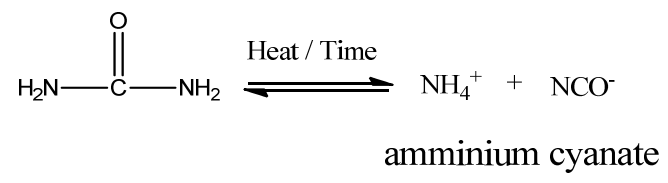

(B) Carbamylation (N-terminus, lysine, arginine or cysteine)

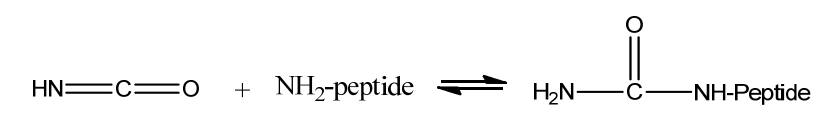

Isocyanic acid

Carbamylated peptide

Figure 2.9. Formation of carbamylated proteins derived from urea. (A) A simple decomposition reaction of urea to form ammonia and cyanic acid (or isocyanic acid). (B) Carbamylation of proteins and peptides as isocyanic acid reacts with the $\mathrm{N}$-terminus, lysine, arginine or cysteine residue.

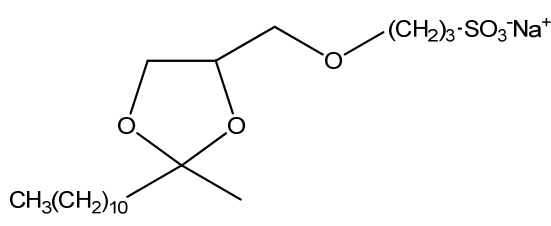

RapiGest $^{\mathrm{TM}} \mathrm{SF}$<smiles>CCCCCCC(C)=O</smiles>

Water immiscible compound
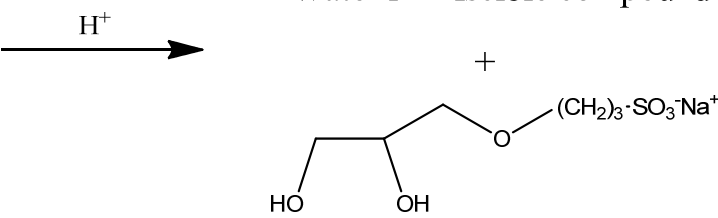

Sulfonic sodium salt

Figure 2.10. Decomposition of RapiGest ${ }^{\mathrm{TM}}$ SF in acid solution to form water immiscible compound and sulfonic sodium salt.

Since the surfactant deeply influenced the selectivity and sensitivity of $\mathrm{TiO}_{2}$ beads, we tested $\mathrm{C} 18$ and $\mathrm{NH}_{2}$ materials for removal of the surfactant prior to $\mathrm{TiO}_{2}$ to get more specificity and efficiency of enrichment. C18-based extraction method had been widely used in proteomics and other analyses for concentrating and desalting peptides [108, 109]. Only 44 and 31 phosphopeptides were identified by LC-MS/MS after desalting with C18 material and subsequent enrichment with $\mathrm{TiO}_{2}$ using RapiGest ${ }^{\mathrm{TM}} \mathrm{SF}$ and urea, respectively (Figure $2.8 \mathrm{C}$ and $2.8 \mathrm{~J}$ ). Thus, the specificity of phosphopeptide enrichment was decreased from $68.4 \%$ to $57.9 \%$ in RapiGest ${ }^{\mathrm{TM}}$ SF experiment, and from $41.5 \%$ to $20.5 \%$ in the urea experiment. The missed phosphopeptides may be too hydrophilic or stuck in eppendorf and pipette tip during 
the desalting step. The removal of sulfonic sodium by $\mathrm{C} 18$ material caused the decrease in specificity of $\mathrm{TiO}_{2}$ enrichment.

Alternatively, we tested for removal of the surfactant with $\mathrm{NH}_{2}$ material based on hydrophilic interaction and anion exchange [110]. The $\mathrm{NH}_{2}$ material in acid solution keeps its positive charge and can capture acid peptides including phosphopeptides. Consequently, the specificity of detected phosphopeptides was increased from $76 \%$ to $86 \%$ in this particular experiment (compared Figure 2.8B with 2.8D) and also in most of the following experiments from $44.9 \%$ to $75.3 \%$ (Figure $2.8 \mathrm{E}$ and $2.8 \mathrm{G}$ ) when $\mathrm{NH}_{2}$ material was used for desalting RapiGest ${ }^{\mathrm{TM}} \mathrm{SF}$. Although $\mathrm{NH}_{2}$ material increased the selectivity of enrichment, an additional desalting step decreased the sensitivity. In urea digested sample, we found that the number of detectable phosphopeptide was decreased when $\mathrm{NH}_{2}$ material was used for desalting (compare Figure $2.8 \mathrm{I}$ to $2.8 \mathrm{~K}$ ). We observed highly carbamylated peptides also bound to $\mathrm{NH}_{2}$ material due to the formation of hydrogen bond between two amine groups; hence, the combination of urea and $\mathrm{NH}_{2}$ material caused a serious problems for the specificity of phosphopeptide detection also in the following experiments (Figure $2.8 \mathrm{~K}$ and $2.8 \mathrm{~N}$ ). In both the experiments we observed a loss of phosphopeptides due to the additional desalting step. Removal of the surfactant with $\mathrm{C} 18$ or $\mathrm{NH}_{2}$ materials prior to $\mathrm{TiO}_{2}$ enrichment showed no improvement for detection of phosphopeptides. In brief, simplified the entire procedure was required to minimize loss of phosphopeptides.

Furthermore, we also evaluated different loading and washing conditions for enrichment of phosphopeptides. Recent studies demonstrated that both the two variant aromatic carboxylic acids, 2,5-dihydroxybenzoic acid (DHB) [75] and phthalic acid (PA) [88], help to decrease the number of non-phosphorylated peptides bound to $\mathrm{TiO}_{2}$ and thus increases the number of detectable phosphopeptides in the sample. Indeed, loading digested samples on $\mathrm{TiO}_{2}$ beads solely in $80 \%$ ACN (v/v), $5 \%$ TFA (v/v) without DHB and PA as additives demonstrates that too many non-phosphorylated peptides bind to $\mathrm{TiO}_{2}$ that were subsequently eluted in the final enrichment step although no phosphopeptides are detected in the flow through or in the wash [111]. We tested whether PA could increase the number of detectable phosphopeptides in our samples when it was used in the loading buffer instead of DHB (Figure 2.8E-G and 2.8L-N). The results show that irrespective from the applied desalting strategy PA does not significantly increase the number of detectable phosphopeptides (compare Figure 2.8B-D to $2.8 \mathrm{E}-\mathrm{G}$ and $2.8 \mathrm{I}-\mathrm{K}$ to $2.8 \mathrm{~L}-\mathrm{N})$.

In summary, the experiments with the $\mathrm{TiO}_{2}$ microspin column show that the use of RapiGest ${ }^{\mathrm{TM}}$ $\mathrm{SF}$ is advantageous for denaturing sample prior to digestion and subsequent $\mathrm{TiO}_{2}$ enrichment of phosphopeptides as compared to urea. Furthermore, an additional desalting step prior to $\mathrm{TiO}_{2}$ enrichment does not significantly improve the detection of phosphopeptides. Although the 
use of $\mathrm{NH}_{2}$ for desalting enhances the purification efficiency of phosphopeptides (percentage phosphopeptides to non-phosphorylated peptides) the overall number of phosphopeptides and thus phosphorylated proteins is lower. For the following experiments, all samples were digested in the presence of RapiGest ${ }^{\mathrm{TM}} \mathrm{SF}$ without any desalting step prior to phosphopeptide enrichment with $\mathrm{TiO}_{2}$ microspin column to avoid any loss of phosphopeptides (Experiment Section 2.3.7, Procedure A).

\subsubsection{Global Profiling of Phosphopeptides from A Crude Mixture of Nuclear snRNP Particles, Individual U snRNP, Spliceosomal Complexes and SR Proteins}

The procedure was first applied on a crude mixture of small nuclear ribonucleoprotein (total $U$ snRNPs) immunopurified from nuclear extraction in order to define a robust generally applicable protocol towards the detection of phosphopeptides derived from proteins involved in eukaryotic mRNA splicing. The snRNPs are particles that combine with pre-mRNA and various proteins to form the key components of the spliceosome, which catalyses the excision of introns and ligation of exons of eukarytic pre-mRNAs

$50 \mu \mathrm{g}$ total $U$ snRNPs were either separated by SDS-PAGE, cut into twenty gel slices and subsequently proteolyzed with trypsin or performed in solution enzymatic digestion with RapiGest ${ }^{\mathrm{TM}}$ SF as mentioned above. The resulting peptides were enriched using the $\mathrm{TiO}_{2}$ microspin columns without any desalting step. Enriched samples were subjected to LC-online coupled ESI-MS/MS or to LC-offline MALDI-MS/MS. The LC-MS/MS analyses were performed in triplicate. The overall workflow is outlined in Figure 2.11.

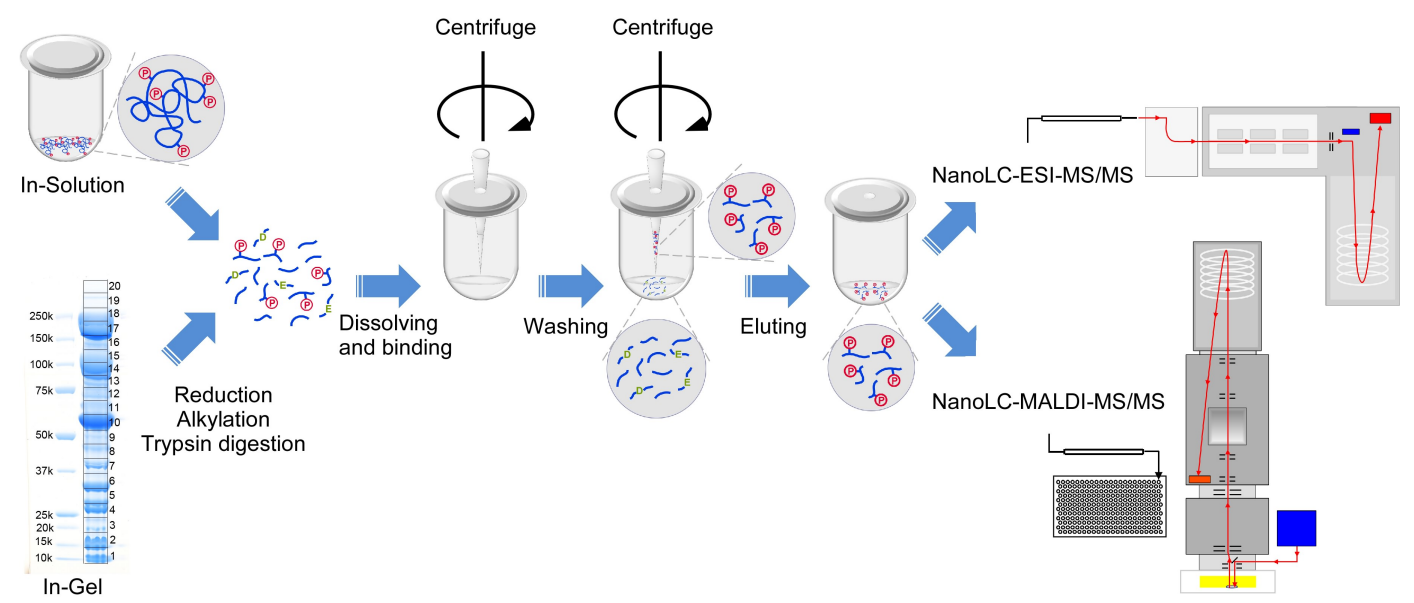

Figure 2.11. Strategy used for the large-scale identification and characterization of phosphorylated sites from total U snRNPs. $\mathrm{TiO}_{2}$ microspin columns were integrated with nanoLC ESI-MS/MS and off-line nanoLC MALDI-MS/MS to identify phosphopeptides derived from in-gel and in-solution proteolytic digestion. 
For the samples derived from in-gel digestion, 60 min LC gradient for separation of (phospho)peptides was applied. In-gel digested samples were not subjected to LC-offline MALDI MS/MS as the overall analysis time of samples (LC off-line and MS and MS/MS on the selected precursor) would exceed a certain threshold that is considered to be reasonable for a routine analysis of phosphopeptides. In-solution digested samples were analyzed by $120 \mathrm{~min}$ and 240 min LC gradients (see Experiment Section 2.3.8 in detail). Figure 2.12 summarizes the results.
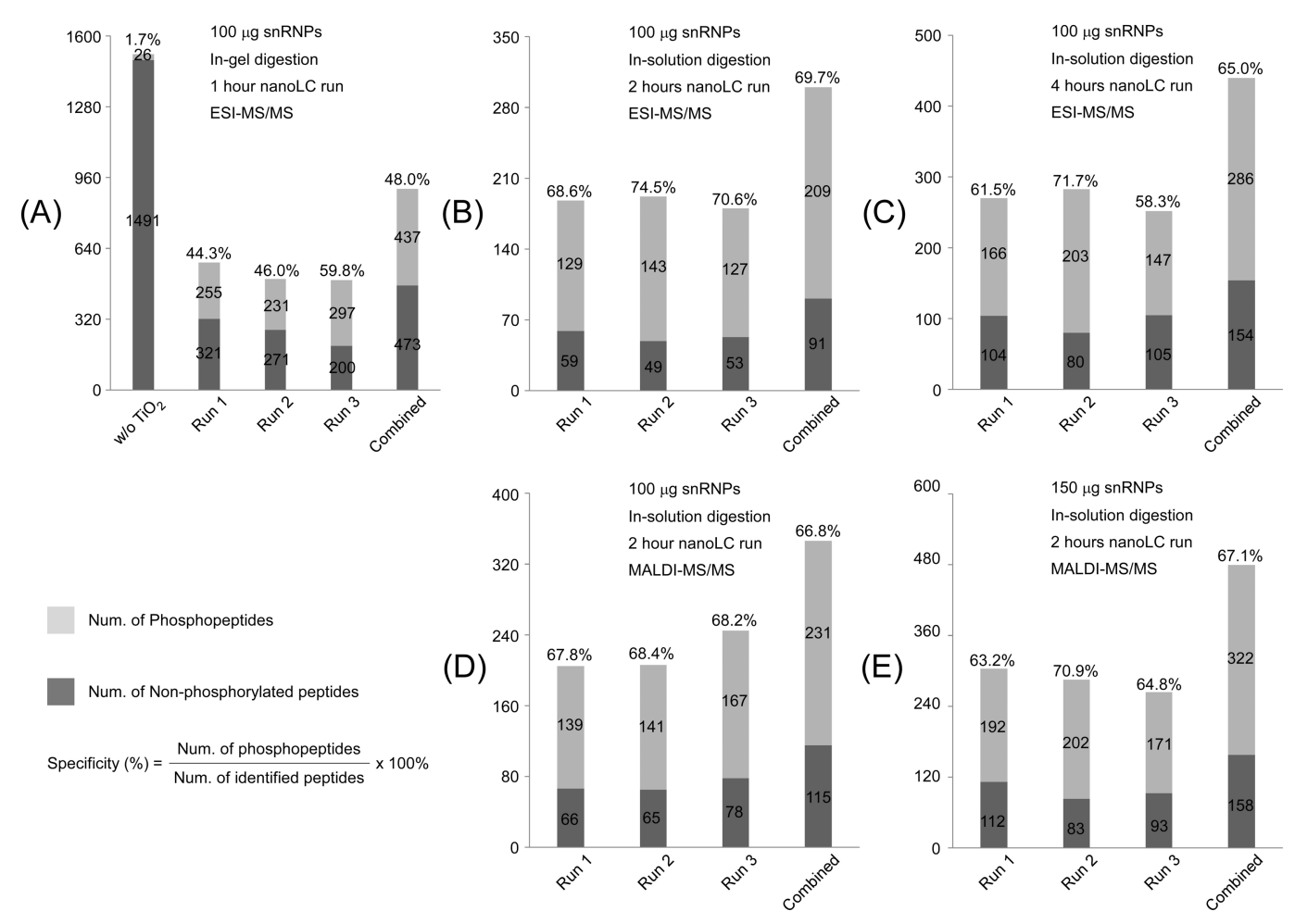

Figure 2.12. The Number of non-phosphopeptides and phosphopeptides identified from tryptic peptides of total U snRNPs by different proteomic approach. (A-D) 100 $\mu \mathrm{g}$ total $U$ snRNPs. (A) In-gel digestion, 1 hour nanoLC gradient, ESI-MS/MS analysis. (B) In-solution digestion, 2 hours nanoLC gradient, ESI-MS/MS analysis. (C) In-solution digestion, 4 hours nanoLC gradient, ESI-MS/MS analysis. (D) In-solution digestion, 2 hours noaoLC gradient, MALDI-MS/MS analysis. (E) $150 \mu \mathrm{g}$ total $U$ snRNPs, in-solution digestion, 2 hours nanoLC gradient, MALDI-MS/MS analysis.

Without enrichment, only 26 phosphopeptides out of 1491 non-phosphorylated peptides were found in in-gel digested sample (Figure 2.12A). Enrichment of the extracted peptides from each gel slice using the $\mathrm{TiO}_{2}$ microspin columns and analysis by LC-online-ESI-MS/MS with a 1 hour gradient identified 437 phosphopeptides in 164 different proteins. From three independent LC separations, approximately $48 \%$ of the totally enriched and sequenced peptides were identified as being phosphorylated. This value is lower as compared to the initial 
studies with the in-solution digest where approx. $68.4 \%$ of the sequenced peptides were phosphopeptides (Figure 2.8B).

In solution digest of the same sample reduced the sample preparation and analysis time, but on the other hand the number of detected and sequenced phosphopeptides was significantly decreased as compared to in-gel digestion. By applying a 2 or 4 hours LC gradient, 209 and 286 phosphopeptides, respectively were detected and sequenced (as compared to 443 in the in-gel digestion). A longer gradient thus helps to identify more phosphopeptides but also more non-phsophorylated so that the purification efficiency is lower. (65\% compared to approx. 69.7 $\%$, compare Figure 2.12B and 2.12C).

We directly applied the enriched sample to off-line nanoLC MALDI-MS/MS and obtained a similar result as compared with ESI-MS/MS (compare Figures 2.12B to 2.12D). Because the selectivity of our enriched procedure was about $70 \%$, the amount of enriched phosphopeptides was sufficient to apply $\alpha$-cyano-4-hydroxy-cinnamic acid (CHCA) as MALDI matrix instead of DHB with phosphoric acid to obtain desirable fragmentation pattern for phosphosite mapping. DHB matrix was shown to enhance the enhance the phosphopeptide signal in MALDI-MS [112], it was nonetheless difficult to generate satisfying MS/MS spectra in MALDI [46]. Increasing the sample amount helps to identify additional phosphopeptides (still with approx. $67.1 \%$ enrichment efficiency) as shown for the LC-offline nanoLC MALDI MS/MS (Figure 2.12E)

Even longer nanoLC gradient (4 hours) applied on in-solution digested samples or larger sample amount did not yield numbers of phosphopeptides comparable to the in-gel digestion with subsequent enrichment on $\mathrm{TiO}_{2}$ microspin columns. On the other hand the selectivity (i.e. the phosphopeptide enrichment efficiency) is higher when samples are digested in-solution using RapiGest ${ }^{\mathrm{TM}} \mathrm{SF}$ as surfactant. At least in our hands, it seems that in-gel digestion reduces the complexity of samples more and thus more phosphopeptides are identified as longer in nanoLC gradients. The design of the $\mathrm{TiO}_{2}$ microspin column makes it possible that phosphopeptides from 24 gel-splices can be enriched in a reasonable time span in a semi high-throughput manner. Importantly, the number of phosphopeptides always varies in the three independent LC separations (extracted peptides from in-gel digestion and peptides derived from in-solution digest). Such phenomenon was described in previous studies with larger proteomes [113] but it was not expected to be similar in our studies with a nuclear sub-proteome, i.e. total snRNP particles.

Figure $2.13 \mathrm{~A}$ summarizes the number of phosphopeptides that were identified by applying our enrichment approach on large scale purified snRNPs. It is obvious that the combination of in-gel digestion, $\mathrm{TiO}_{2}$ microspin and LC-ESI-MS/MS in triplicate yield the greatest number of phosphopeptides. Figure 2.13B compares the total number of phosphopeptides and 
phosphoproteins identified in the three different approaches (in-gel-digestion, LC-online ESI-MS/MS, and LC-offline MALDI-MS/MS). Notably, each digestion, separation and MS method identified different, partially overlapping segments of the phosphopeptides and phosphoproteins, implying that none of the methods by itself is currently able to comprehensively analyze a phosphoproteome. By combining the results, a total of 717 phosphopeptides were identified from crude mixture of immunoaffinity purified total U snRNPs. In 641 phosphopeptides the phosphorylation site could be unambiguously identified thus confirming 510 distinct phosphorylation sites. 115 phosphopeptides in 61 phosphoproteins were found in all three approaches. 228 phosphopeptides from 69 proteins were identified after in-gel digestion only, 120 phosphopeptides from 41 protein were identified by in-solution digestion and subsequent ESI-MS/MS only, and 78 phosphopeptides from 25 proteins were identified by in-solution digestion and subsequent LC-offline MALDI-MS/MS only. The number of non-confirmed phosphopeptides is relative low in either approach (Figure 2.13A) since the MS/MS spectra were manually evaluated. We also searched the MS/MS spectra against a decoy database to determine the false positive rate less than $1 \%$.

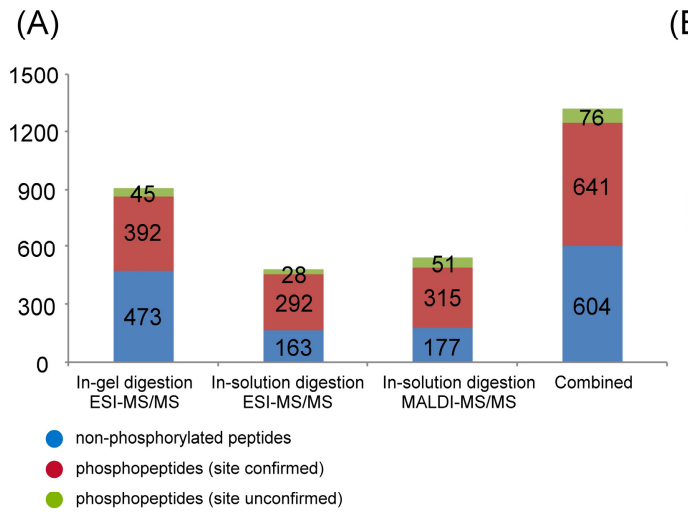

(B)

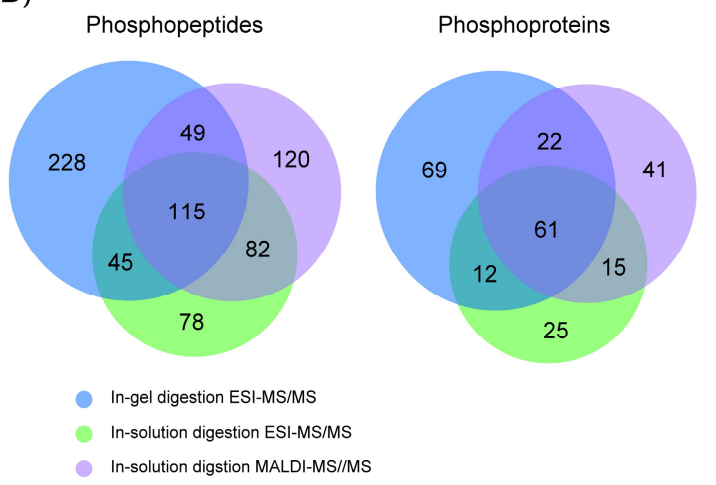

Figure 2.13. (A) The number of non-phosphopeptides and phosphopeptides identified from tryptic peptides of total U snRNPs by different proteomic approach. (B) Overlap of distinct phosphopeptides and phosphoproteins identified with ESI- and MALDI-MS/MS integrated with $\mathrm{TiO}_{2}$ microspin column enrichment from in-gel and in-solution digested sample.

Taking ESI-MS/MS and MALDI-MS/MS as comparison, the latter seemed laborious to localize the position of phosphorylated site. Approximately $14 \%$ (51/366) of phosphopeptides we could not ascertain the location because the molecular ions corresponding to these phosphopeptides offered high abundance of neutral loss of phosphate moiety (-98 $\mathrm{Da}$ ) in MALDI-MS/MS (Fig. 2.14B and 2.14D). Compared the MS/MS spectra of phosphopeptide ${ }^{54}$ CAPSAGpSPAAAVGR ${ }^{67}$, it was straightforward to confirm the site at $S 60$ relied on $\mathrm{y}_{7}, \mathrm{y}_{8}-98$, $\mathrm{y}_{9}$ ions from ESI-MS/MS spectrum. (Figure. 2.14A) However, in MALDI-MS/MS spectrum, $\mathrm{y}_{7}$ and $\mathrm{y}_{12}$ ions indicated two possible sites at S57 or S60 (Figure 2.14B); nevertheless, we still 
had large amount of high quality spectra, like phosphopeptide ${ }^{392}$ FNDpSEGDDTEETEDYR ${ }^{407}$ could distinctly confirm the site at S395 in both systems (Fig. 2.14C and 2.14D). In brief, our $\mathrm{TiO}_{2}$ enrichment procedure in combination with different proteomic strategy successfully applied to large scale phosphoproteome analysis, resulting in the identification of 245 phosphoproteins corresponding to 510 distinct phosphorylated sites in total U snRNPs.

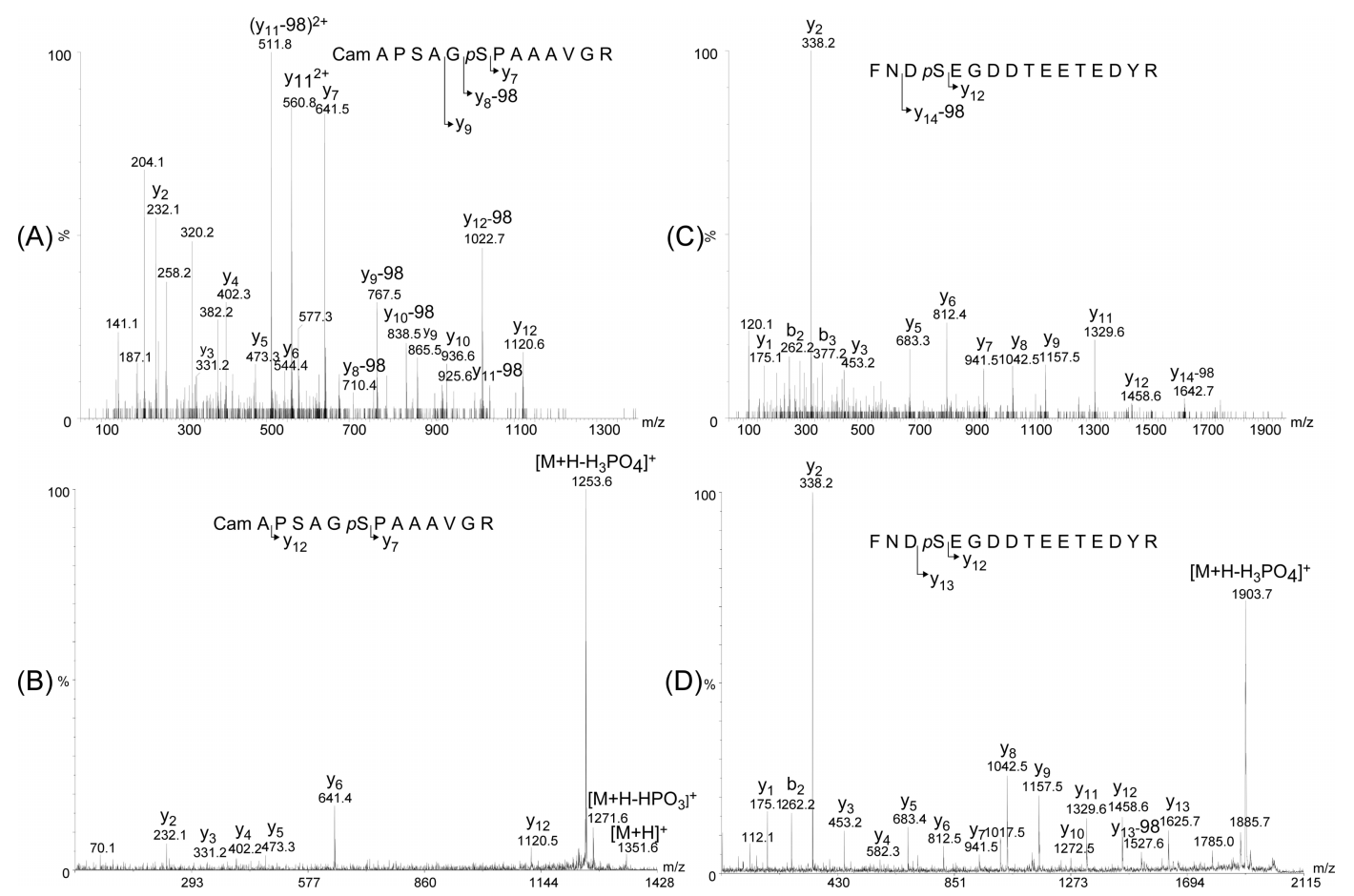

Figure 2.14. MS/MS spectra of phosphopeptides extracted from tryptic peptides of total U snRNPs. (A) and (B) phosphopeptide ${ }^{54}$ CAPSAGpSPAAAVGR ${ }^{67}$ detected with ESIand MALDI-MS/MS, respectively. (C) and (D) phosphopeptide ${ }^{392}$ FNDPSEGDDTEETEDYR ${ }^{407}$ detected with ESI- and MALDI-MS/MS, respectively.

To examine the power of our enrichment for identification of phosphorylation sites, we further applied our strategy on the large scale analysis of individual U snRNPs, SR Proteins and spliceosomal complexes. Direct $\mathrm{TiO}_{2}$ microspin columns integrated with nanoLC ESI-MS/MS analyses of tryptic peptides from protein complexes in solution as well as gel bands from one-dimensional SDS-PAGE afforded the best combination in efficient construction of phosphorylation map as showed in Figure 2.15. 1381 distinct phosphorylated sites corresponding to 390 phosphoproteins corresponding to were identified in the study (Appendix 1). In the 1381 phosphorylation sites, $84.1 \%$ (1161) and $14.6 \%$ (202) were serine and threonine, respectively, and only $1.3 \%$ (18) were tyrosine. The above values were similar to the results from previous reports [57]. Manually validated phosphorylation sites in the corresponding proteins were searched against Expasy Knowledgebase (http://us.expasy.org/) and PhosphoSite database (http://www.phosphosite.org/) [114]. Both these database have 
collected comprehensive information on in vivo protein phosphorylation and their corresponding references. In previous report, 2002 phosphorylation sites corresponding to 967 proteins were identified in large scale nuclear extracted proteins by Beausoleil et al. [65], however, many of the phosphorylated sites identified in this study have not been reported by Beausoleil et al.. Approximately $46.3 \%$ of the sites (640 phosphorylated sites) were considered to be novel.

$(\mathrm{A})$

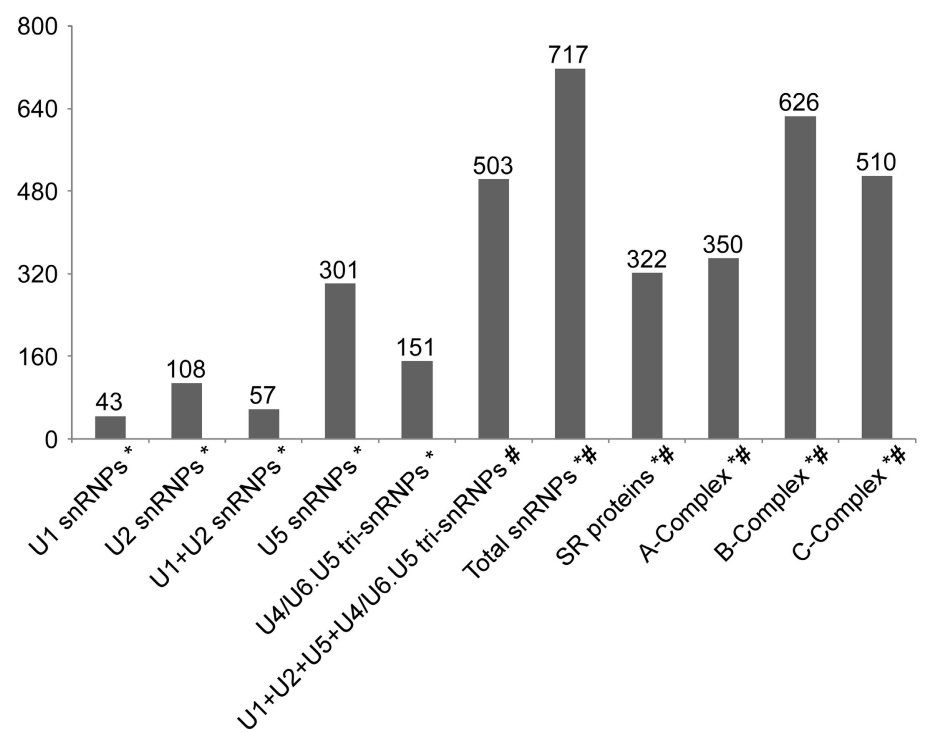

(B)

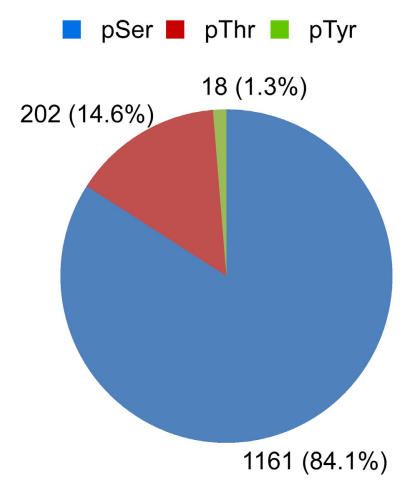

Figure 2.15. (A) Number of phosphopeptides identified from Individual $U$ snRNPs, total snRNPs, SR proteins, spliceosomal complexes. * means in-solution digestion. \# means in-gel digestion from SDS-PAGE. (B) The distribution of phosphorylated residues identified from spliceosomal proteins .

\subsubsection{Novel and Known Kinase Motifs in Spliceosomal Proteins}

After we generally classified all phosphopeptides in our data set into primary sequence categories, we sought to further refine these categories into specific, frequency-corrected phosphorylation motifs. Kinase specificity typically depends on the primary amino acid sequence surrounding the target phosphorylation site [115]. Peptide sequences for phosphorylated sites localized on serine and threonine were all aligned, and their lengths were adjusted to \pm 6 amino acid from the central position and submitted to the Motif-X algorithm [100]. Each identified motif, logo-like representations were created to graphically display. These logos included not only the residues strictly discovered to be part of the motif, but also the frequencies of all additional adjacent amino acids as showed in Figure 2.16. The classes included Pro-directed, basophilic and acidiphilic, other motifs generated containing a minimum of 20 pSer or pThr occurrences. The majority of the found pSer- and pThr-containing sites 
were Pro-directed which accounted for $12.5 \%$ (145/1161) and $39.1 \%$ (79/202) of all detected phosphorylation, respectively, as showed in Figure 2.16A. Another class are acidic motifs in which acidic residues (Asp or Glu) were abundant on the C-terminus (pSDxE, pSExE, pSxxE, pSxxD, DpSD and pSxExE motifs were shown in Figure 2.16B, respectively). In total 223 sites out of 1161 pSer (19.2\%) were identified with such a consensus motif. These sites are previously described to be a target for casein kinase (like) II [100].

(A)

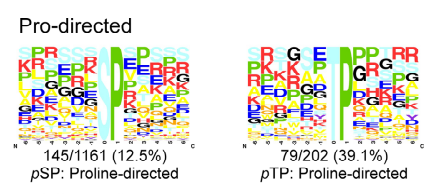

(B)

Acidic molirs

(C)
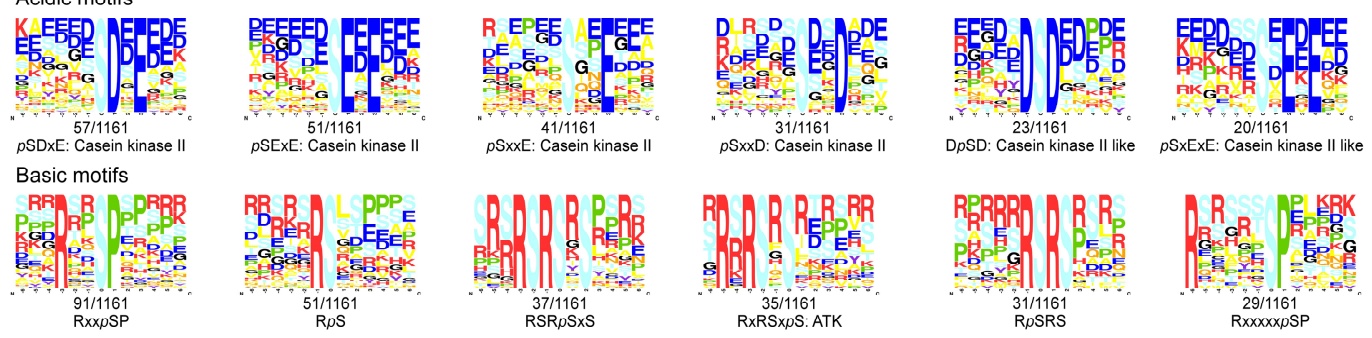

(D)
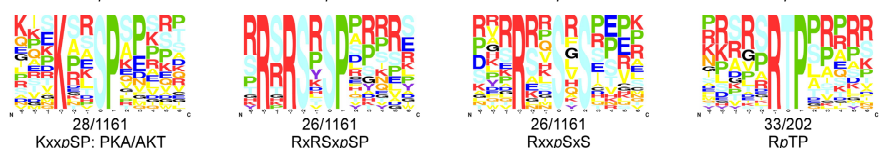

Other motifs
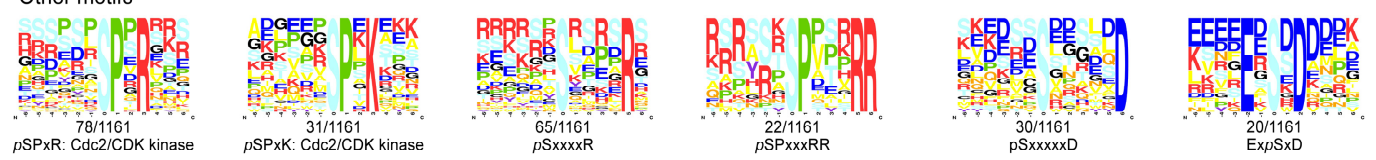

Figure 2.16. Phosphorylation-specific motifs using the Motif-X algorithm. (A-D)

Sequence logos of phosphorylation motifs where the phosphorylated residue (Ser or Thr) is centered. Number of occurrences of a particular motif in 1161 unique serine and 202 unique threonine phosphorylation are indicated. (A) Pro-directed motif with a strong favorite for Pro residue next to C-terminal of Ser and Thr residues, these motifs were observed 145 and 79 occurrences, respectively. (B) Representation of acidic motifs of casein kinase II substrate (like) with 57, 51, 41, 31, 23 and 20 occurrences, respectively. (C) Representation of basic motifs of Ser and Thr residues were detected with 354 and 33 occurrences, respectively. ATK referred to as protein kinase $B$ or rac protein kinase. PKA referred to as protein kinase $A$. (D) Representation of other motifs of Ser residues were detected 246 occurrences. Cdc2/CDK kinase substrate motif $\mathrm{pSPxR}$ and $\mathrm{pSPxK}$ were observed 109 times.

Interestingly, basic motifs in which basic (Arg or Lys) residues are abundant on the $\mathrm{N}$-terminal site, RSRpSxS, RxRSxpS and RpSRS were found 37, 35 and 31 times, respectively, in SR domains of so-called SR proteins. RxRSxpS had been described to be the consensus motif for ATK as showed in Figure 2.16C [100]. SR proteins facilitate the association of snRNPs with the pre-mRNA during spliceosome assembly, and phosphorylation is essential for SR protein 
activity $[116,117]$ and enhances their interactions with other spliceosomal proteins [118]. A hitherto uncharacterized motif, RxxpSP, was found in 91 sites and appeared to be a combination of both basophilic (RxxpS, PKA/PKC kinase substrate motif [119]) and proline-directed (pSP) motifs. Cdc2/CDK kinase motif pSPxR and pSPxK appeared 78 and 31 sites, respectively, shown in Figure 2.16D [100, 120]. Only eighteen pTyr sites were identified in this study, hence, the data set was significantly less than that for Ser or Thr to find the corresponding motif by alignment.

\subsubsection{Application of $\mathrm{TiO}_{2}$ Microspin Column}

We further examine the power of our $\mathrm{TiO}_{2}$ microspin column. We collaborated with Marc Schneider et al. in Prof. Reinhard Lührmann's Laboratory on the project "Human PRP4 kinase is required for stable tri-snRNP association during spliceosomal B complex formation" and published on Nature Structural \& Molecular Biology, 2010 [121]. Two previously unidentified phosphoprotein, namely the pre-mRNA processing factors PRP6 and PRP31, were discovered as showed in Figure 2.17. The purified B complex was separated by SDS-PAGE and stained with Coomassie blue. The proteins of interest were excised from gel, digested with trypsin, extracted and dried down with Speedvac. The resulting tryptic peptides were enrichment with our $\mathrm{TiO}_{2}$ microspin columns and followed by nanoLC ESI-MS/MS analysis. Human PRP6 and PRP31 were phosphorylated at multiple sites after tri-snRNP incorporation in to the spliceosomal B complex. In human PRP6, we identified pSer at positions 263 and 279, and pThr at positions 205, 266 and 275. In human PRP31, we detected pSer at positions 439 and 498, and pThr at positions 440, 448 and 455. At Ser 445, Ser 446, Ser 450 and Ser 451, the exact positions were not assigned due to their close proximity but the MS and MS/MS indicated that two of the four serine residues were phosphorylated, respectively (Appendix 2). Marc Schneider et al. provide the evidence that both proteins are directly phosphorylated by PRP4 kinase, which is required for the assembly of stable, functional B complex. They suggest multiple phosphorylation events on PRP6 and PRP31 assist to stabilize the interaction of the tri-snRNP during the B complex assembly and the splicing can potentially be modulated at multiple regulatory checkpoint. 
$\begin{array}{lcllll}\text { PRP6 } & 205 & 263266 & 275 & 279\end{array}$

201 DPRQTQFGGL NTPYPGGLNT PYPGGMTPGL MTPGTGELDM RKIGQARNTL MDMRLSQVSD SVSGQTVVDP KGYLTDLNSM IPTHGGDIND IKKARLLLKS 300

PRP31

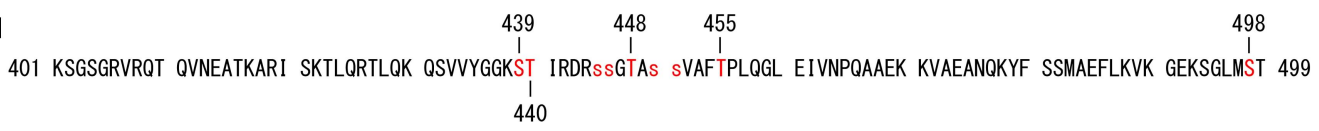

Figure 2.17. Partial amino acid sequence of human PRP6 (aa 201-300) and PRP31 (aa

401-499 and 495-499). Red capital letters indicated phosphorylation sites identified in this study. Red lowercase letters indicated the exact location of phosphorylation sites could not be assigned.

We collaborated with Thomas Oellerich et al. in Prof. Jürgen Wienands's Laboratory on the project "SLP-65 Phosphorylation Dynamics Reveals a Functional Basis for Signal Integration by Receptor-Proximal Adaptor Proteins" and published on Molecular \& Cellular Proteomics, 2009 [122]. SLP-65 serves functions as a central linker protein that bridges kinases associated with the $\mathrm{B}$-cell receptor $(\mathrm{BCR})$ with a multitude of signaling pathways, regulating biological outcomes of B-cell function and development. A total of 41 phospho-acceptor sites within SLP-65 were identified under BCR stimulation as showed in Figure 2.18A. The exact location of amino acid were 36 residues; of these, $21 \mathrm{pSer}, 6 \mathrm{pThr}$ and $9 \mathrm{pTyr}$ residues were found. For phosphorylation sites located within Ser 397 and Ser 398 and between the amino acids 422 and 429 , the exact positions were not assigned due to their close proximity but the MS and MS/MS indicated that one of the two and four of the six serine residues were phosphorylated, respectively. We further integrated SILAC (stable isotope labeling with amino acids in cell culture) approach for profiling phosphorylation dynamics of SLP-65 in resting and activated B cells with BCR stimulation for 2 or $20 \mathrm{~min}$. The most frequently detected phosphopeptides ${ }^{168}$ KPSLPSPAAKPK $^{179}$ at Ser 170 and Ser 173 were observed (Figure 2.18B-E) The quantity of the singly phosphorylated peptide decreased slightly upon BCR stimulation (Figure 2.18B), whereas the quantity of the doubly phosphorylated peptide increased over time (Figure 2.18C). 'The MS/MS spectrum of the singly phosphorylated peptide contained an exclusive phosphorylation at Ser 173 but not at Ser 170 (Figure 2.18D). The MS/MS spectrum of the doubly phosphorylated peptide indicated phosphorylated at Ser 170 and Ser 173. Taking cue together, the amount of the singly phosphorylated peptide at Ser 173 reduced because the formation of phosphorylation at Ser 170 converted the singly phosphorylated peptide into its doubly phosphorylated peptide under BCR stimulation. The result indicated BCR stimulation acted mainly on Ser 170 . In brief, 23 distinct phosphopeptides corresponding to 24 phosphorylation sites were quantified. Our data showed that the phosphorylation of SLP-65 acted as early signal initiation and later signal processing during the BCR stimulation. 
MDKLNKLAVP AGEKFRKLQK MVHDIKKNES GI INKFKKFQ NEQVALICKT GKDTWDRLKK KPPPSLPRRD YASEHADNEE EQWSDDFDSD YENPDGHSDS
EMYVVPSEEN PDDSYEPPPS EQEKKKIPSS FPISRGEYAD NRTSHHQLPP INKPLPSTPS SALPRPKKPS LPSPAAKPKL PLKPRECSDD EDNYIVPVD

A) 201 DDDNYIEPTE SSTPPPAKPP VNRFMKPPAK SALPTPPKPS LASDMQEVYE VPEEEEELSP PPVTRFTKPL PATRAONAEH SHMHSMTRES PKLDASRNIL

301 PLPRNRLHPK TDHEANNNDE NHSFSNTQES KFPPGAAPSP LPRALKKTSN AVNPAKPCLP SRDTFTVNED KPTAADRRRG SSHEFPLPPL PSGTPKSSLQ 401 KPLVLPKVPE APSRALGTSP HSSIsSISST ADQDAGVHSK AWYAATCDRK TAEDALYRSN KDGSFLIRKS SGQDSRQPYT LVVFYNRRVY NIPIRFIEST 501 RQYALGREKC GEERFDSVAE IVENHQHTSL VLIDSQNNTK DSTKLKYIVR VS
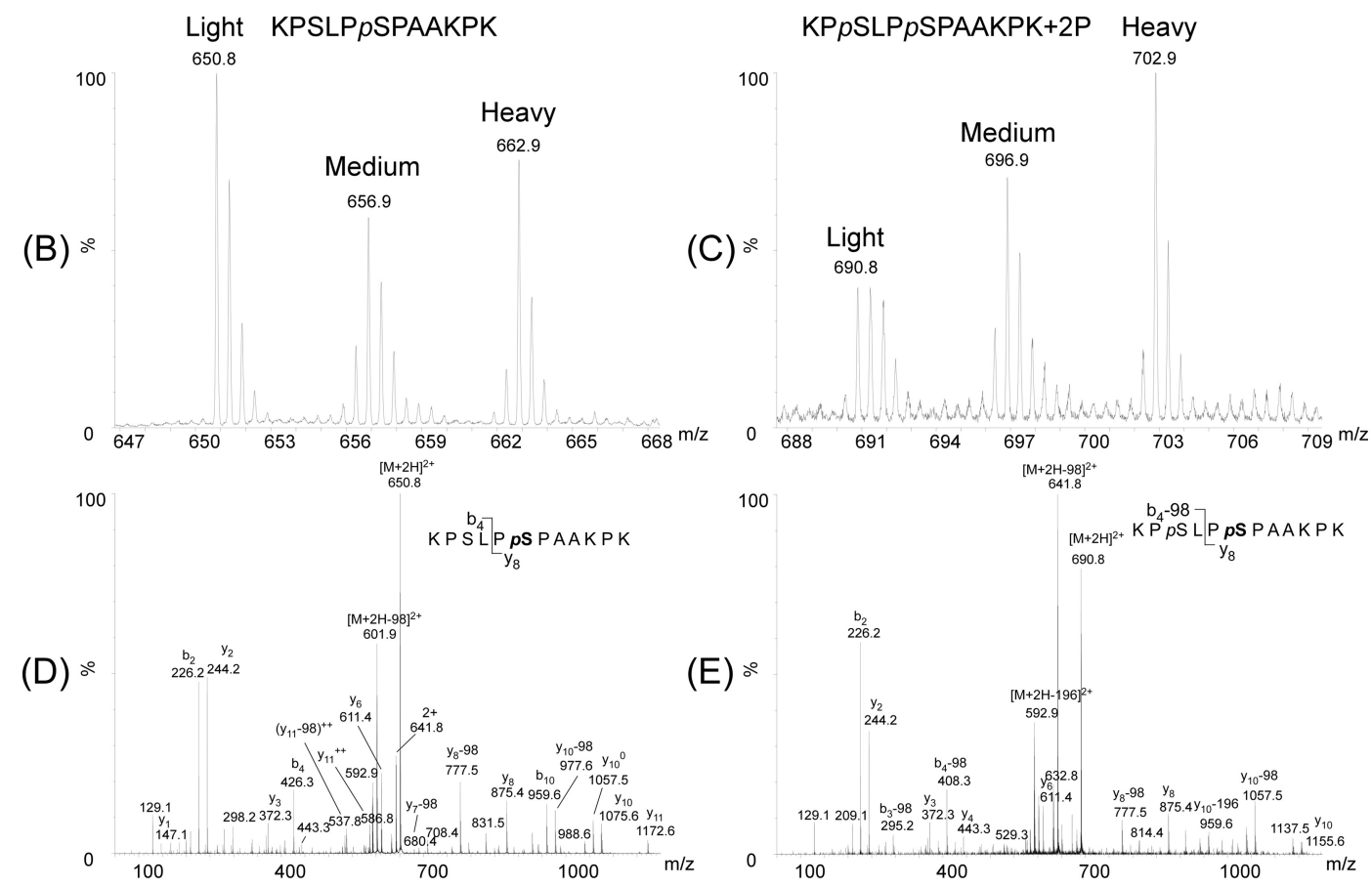

Figure 2.18. (A) The phosphorylation sites in SLP-65 from gallus gallus. Red capital letters indicated phosphorylation sites identified in this study. Red lowercase letters indicated the exact location of phosphorylation sites could not be assigned. (B)-(E) Stable isotope labeling with amino acids in cell culture (SILAC) approach for relative quantification of the most frequently detected phosphopeptide ${ }^{168}$ KPSLPSPAAKPK ${ }^{179}$ in SLP-65. (B) Mass spectrum of the singly phosphopeptide at Ser 173 in unstimulated cells and in BCR stimulation for 2 or $20 \mathrm{~min}$ obtained at m/z 650.8 (light), 656.9 (medium) and 662.9 (heavy), respectively. (C) Mass spectrum of the doubly phosphopeptide at Ser 170 and Ser 173 in unstimulated cells and in BCR stimulation for 2 or $20 \mathrm{~min}$ obtained at $\mathrm{m} / \mathrm{z} 690.8$ (light), 696.9 (medium) and 702.9 (heavy), respectively. (D) MS/MS spectrum of the singly phosphopeptide ${ }^{168} \mathrm{KPSLPpSPAAKPK}^{179}$ at $\mathrm{m} / \mathrm{z} 650.8$. (E) MS/MS spectrum of the doubly phosphopeptide ${ }^{168} \mathrm{KPpSLPpSPAAKPK}^{179}$ at $\mathrm{m} / \mathrm{z} 690.8$.

We further collaborated with Xiao Luo et al. in Prof. Markus C. Wahl's Laboratory on the project "Structural and Functional Analysis of the E.coli NusB-S10 Transcription Antitermination Complex" and published on Molecular Cell, 2008 [123]. In this study, our $\mathrm{TiO}_{2}$ microspin column in combination with mass spectrometry was employed to map the hitherto non-characterization of UV-induced protein-RNA contact sites in NusB-S10 complex. The identification of contact sites after UV-induced crosslinks is restricted by the relatively low yield of UV-induced protein-RNA crosslinks. Therefore, the most critical step in such analyses is the effective enrichment of crosslinked species from the excess of non-crosslinked species 
prior to MS analysis. A feature shared by protein-RNA crosslinks, free RNA oligonucleotides and phosphopeptides, namely the phosphate groups that these three carry, is used to enrich, as phosphate groups can interact with the $\mathrm{TiO}_{2}$ material through their free lone pair of electrons. NusB or NusB-S10 protein complex was first incubated with RNA oligomer and exposed to UV light to form NusB-RNA or NusB-S10-RNA complex, respectively. Subsequently, the crosslinked complexes were precipitated with $\mathrm{EtOH}$, hydrolyzed with RNases $\mathrm{T} 1$ and $A$, digested with trypsin. The tryptic peptides were enriched with $\mathrm{TiO}_{2}$ microspin column for RNA-peptide crosslinks and followed analysis by nanoLC-ESI-MS/MS. Overall, we identified four peptides in NusB and three peptides in $S 10$ that crosslinked to distinct, short RNA elements as showed in Figure 2.19 and Table 2.1. UV-induced crosslinked reaction in the absence of RNA oligomer and MS analysis with complete protein complex but without UV irradiation did not give rise to any peaks corresponding to those of the identified peptide-RNA crosslinks. As previous report, in some cases we saw non-enzymatic breakage or hydrolysis of crosslinked oligonucleotides during enrichment or in the gas phase of the MS [124]. However, we could not identify the actual crosslinked amino acids by mass spectrometry.
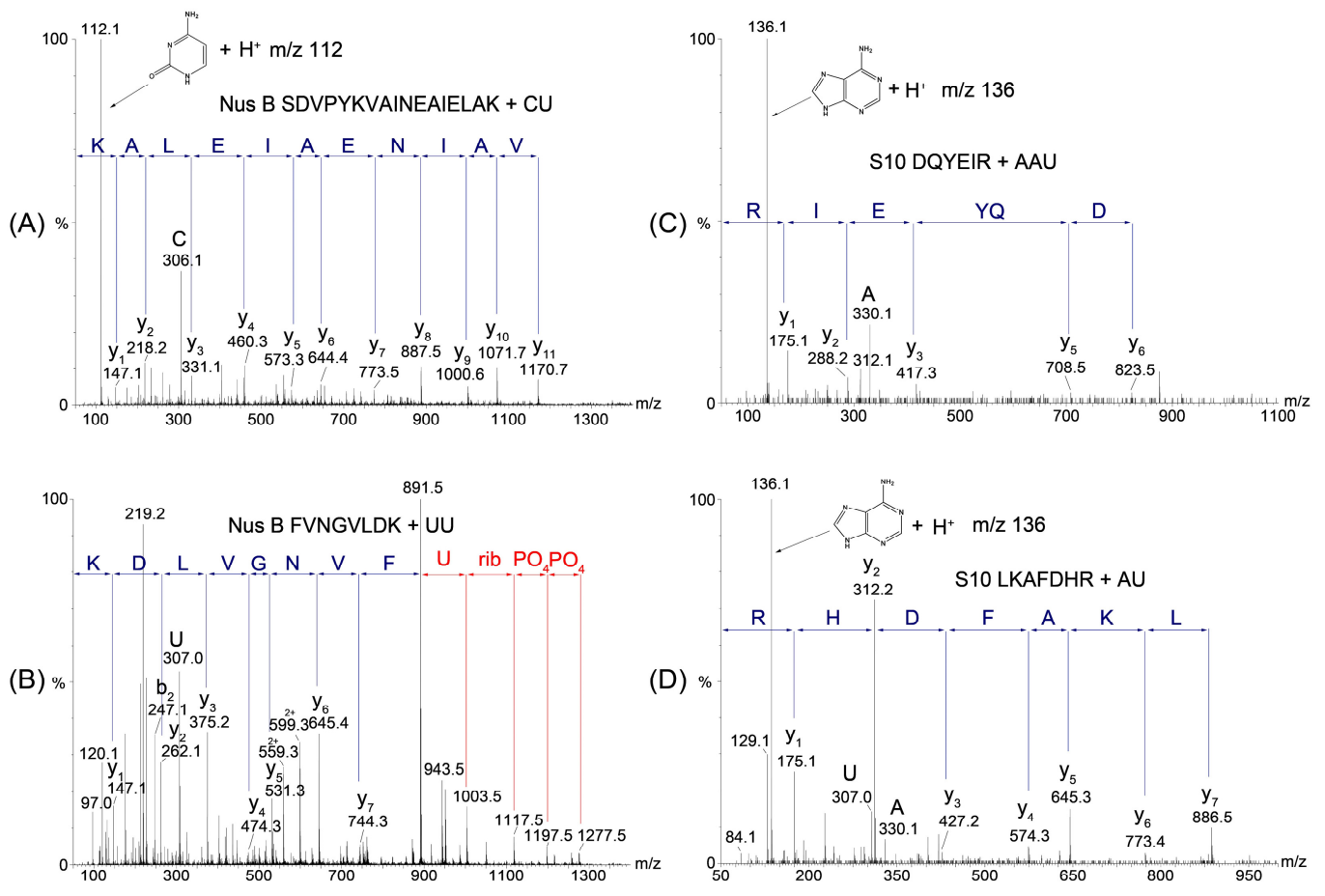

Figure 2.19. MS/MS spectra of protein-RNA crosslinks by $\mathrm{TiO}_{2}$ enrichment. (A-D) Peptide-RNA crosslinks derived from UV-induced NusB-S10- $\lambda$ BoxA RNA complex (19mer ABoxA oligomer, CACCGCUCUUACACAAUUA) are NusB SDVPYKVAINEAIELAK+CU, NusB FVNGVLDK+UU, S10 DQYEIR+AAU and S10 LKAFDHR+UA, respectively, 
Table 2.1. Identification of protein-RNA crosslinks in NusB-S10 complex by $\mathrm{TiO}_{2}$ enrichment in combination with mass spectrometry.

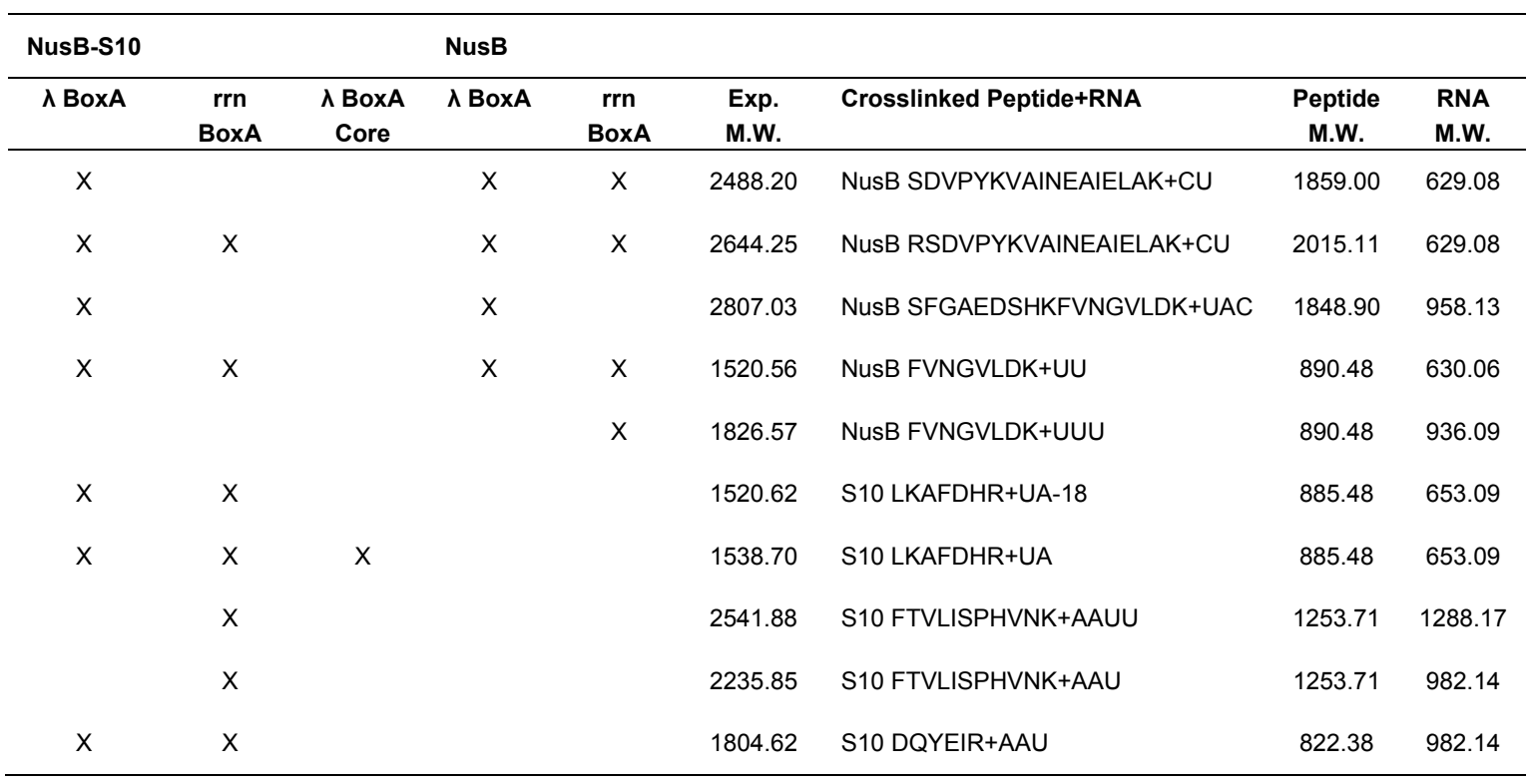

$\lambda$ BoXA: CAC CGC UCU UAC ACA AUU A

rrn BoxA: CAC UGC UCU UUA ACA AUU A

$\lambda$ BoxA core: CGC BrUCU UAC ACA; BrU - 5-bromo uridine

$\mathrm{X}$ indicates observation of a particular crosslink in a given mixture

In brief, mass spectrometric mapping of UV-induced protein-RNA crosslinks revealed that the NusB-S10 complex presents an intermolecular, composite, and contiguous binding surface for RNAs containing BoxA antitermination signals. Our $\mathrm{TiO}_{2}$ enrichment procedure can be applied to any protein-RNA complex for the identification of proteins in direct contact with RNA and for visualization of the site of interaction.

\subsection{Conclusion}

A disposable $\mathrm{TiO}_{2}$ microspin column for isolation of phosphorylated peptides of proteolytically digested proteins was fabricated. The advantages of such $\mathrm{TiO}_{2}$ microspin column include, i) short enrichment and analysis time, ii) ease to use in standard routine applications for phosphopeptide analysis, iii) economy due to low costs, iv) high specificity and sensitivity for the enrichment of phosphorylated peptides from complex sample. It offers further the opportunity, to pack different chromatographic resins (C18, SCX, SAX, IMAC etc.) for different application or mixed resins to integrate several functions in one step prior to mass spectrometric analysis. In comparison with decomposed products of urea, loading sulfonic sodium which hydrolyzed from RepiGest ${ }^{\mathrm{TM}} \mathrm{SF}$ onto the $\mathrm{TiO}_{2}$ microspin column significantly increases the efficiency of phosphopeptide enrichment as sulfonic ion competes for binding sites with acidic amino acid residues in the peptides. An additional SDS-PAGE gel separation 
prior to enrichment increases the number of detectable phosphopeptides. Our $\mathrm{TiO}_{2}$ enrichment procedure in combination with different proteomic strategy was applied to phosphoproteome analysis of spliceosomal proteins, resulting in the identification of 1381 unique phosphorylation sites corresponding to 390 distinct proteins. At least 640 phosphorylation sites identified in this study appear to be novel, which may provide a valuable resource to the biological research community. In brief, we designed a microspin column and optimized a enrichment procedure that allowed us not only to carry out higher-throughput analyses assessing phosphorylation sites of multiple samples in a more efficient time scale but also to map UV-induced peptide-RNA crosslinks by mass spectrometer. 
Chapter 3 - Efficient Enrichment of Intact Phosphoproteins prior to Mass Spectrometric Analysis

\subsection{Summary}

The selective enrichment of specific proteins prior to mass spectrometry (MS) analysis facilitates the identification of lower abundance proteins in current proteomic research. Up to now, only few methods have been described that allow for comprehensive purification of intact phosphoproteins from mixtures. In this study, we have developed a straightforward and reliable pre-fractionation procedure that effectively removes most of the highly abundant non-phosphorylated proteins, and allows for the selectively isolation of low abundant phosphoproteins based on calcium phosphate precipitation (CPP). By monitoring Coomassie blue staining of the model phosphoprotein beta-casein on SDS-PAGE to evaluate the efficiency, optimized sets of $\mathrm{CaCl}_{2} / \mathrm{Na}_{2} \mathrm{HPO}_{4}$ concentration and washing buffer conditions were realized for enrichment. Taking the optimized condition in combined with our in-house titanium dioxide $\left(\mathrm{TiO}_{2}\right)$ microspin column provided highly selective enrichment of phosphopeptides. The U1 small nuclear ribonucleoproteins (U1 snRNPs) were used as the complex samples to demonstrate the feasibility of this approach. Many phosphorylation sites identified in this study appear to be novel, including sites from U1-specific proteins (U1-70K, U1-A and U1-C) and Sm proteins (B'/B, D2, D3 and F).

\subsection{Introduction}

Reversible protein phosphorylation is the most important post-translational modification involved in many regulatory cellular functions such as the regulation of cell cycle, signal transduction, differentiation, proliferation, transformation, and metabolism [56, 57]. Almost $2 \%$ of the human genome encodes protein kinases and an estimated one-third of all proteins in mammalian cells are expected to be phosphorylated [125]. Among the amino acid residues that can be phosphorylated, O-phosphates on serine, threonine, and tyrosine residues are by far the most abundant. The occurrence of phosphorylation on serine and threonine residues is more frequent than on tyrosine residue in a vertebrate cell, with the ratio of pSer/pThr/pTyr in the order of 1800:200:1.8 [57]. The phosphoramidates of arginine, histidine, and lysine also occur as do acyl derivatives of aspartic and glutamic acid, although they are less abundant.

Recently, MS has been widely applied as a powerful tool for the identification of proteins and the site assignment of protein modifications, including phosphorylation, taking the advantage of its speed, high sensitivity, reliability, and capability for determining phosphorylation site by tandem mass spectrometric techniques. However, large scale phosphoproteomic analysis still remains a substantial challenge due to the lower abundance of phosphoprotein, the substoichiometric nature of phosphorylation, and some technical limitations. The presence of 
large amounts of non-phosphorylated peptides in the digestion product also suppresses the ion signal of phosphopeptides in MS detection [58-61]. Therefore, the development of efficient methods for highly specific enrichment of phosphoproteins and phosphopeptides prior to MS analysis becomes a critical step for studying protein phosphorylation.

Notwithstanding the complete separation of the phosphoproteins and phosphopeptides is still a challenging task, the analysis of phosphoproteome is one of the most exciting field in the current proteomic research. Although phosphoproteins can be separated by antibody immunoprecipitation [89, 126-130], the enrichment with anti-pSer, anti-pThr, or anti-pTyr antibodies depends on the affinity and specificity of antibodies, which limits comprehensiveness of the protein phosphorylation. Over the past decade, a variety of methodologies have been developed for phosphopeptide and phosphopeptide enrichment. One of these methods, immobilized metal ion affinity chromatography (IMAC), has been successful applied to enrich not only phosphopeptides but also phosphoproteins in large scale proteomic analysis [67-74]. However, multiple acidic residues of peptides are frequently retained by this procedure, interfering the further MS analysis [89-92]. The denaturing conditions or low pH was used that does not maintain enzyme activity of phosphoprotein. In addition, a promising phosphopeptide enrichment strategy was introduced by Sano et al., in which titanium $\mathrm{TiO}_{2}$ had been used as an alternative to IMAC [75-79]. More recently, CPP had been proven to be very efficient for enriching phosphopeptides from a complex tryptic peptides by Xumin Zhang et al. , in which the phosphopeptides are pulled down by the formation of an insoluble calcium phosphate $[69,131]$. Nevertheless, phosphoproteome studies are still hindered by lacking efficient methods for comprehensive purification of intact phosphoproteins until now.

Here, a straightforward and reliable phosphoprotein purification method was developed based on CPP. It opens up a new approach facilitating large scale phosphoproteomic analysis. The majority of non-phosphorylated proteins were removed by this procedure which then led to an enhanced detection of the lower abundant phosphoproteins. In initial studies, a protein mixture consisting of beta-casein, bovine serum albumin and myoglobin was used as model to optimize and test the procedure. We found that our method is highly tolerable with detergents. In addition, by coupling CPP with our in-house microspin column $\mathrm{TiO}_{2}$ enrichment procedure, we were able to minimize co-enrichment of non-phosphorylated peptides. Finally, we have evaluated our approach on native U1 snRNPs complexes isolated from HeLa nuclear extract. A total of 192 unique phosphorylation sites corresponding to 45 distinct proteins were identified in the glycerol gradient purified U1 snRNPs. In summary, our method paves the way for pre-fractionation of phosphoproteins prior to enrichment of phosphopeptides which will be particularly useful in large scale phosphoproteome studies. 


\subsection{Experiment Sections}

\subsubsection{Materials}

Chloroacetamide (CAA), ammonium bicarbonate, 2,5-dihydroxybenzoic acid (DHB) and trifluoroacetic acid (TFA) were obtained from Sigma-Aldrich (St. Louis, MO). Sequencing grade, modified trypsin was obtained from Promega (Madison, $\mathrm{WI}$ ). Sequencing grade endoproteinase, Asp-N and Lys-C were obtained from Roche (Mannheim, Germany). RNase $\mathrm{T} 1$ and $\mathrm{A}$ were obtained from Ambion (Austin, TX). Calcium chloride $\left(\mathrm{CaCl}_{2}\right)$, disodium hydrogen phosphate $\left(\mathrm{Na}_{2} \mathrm{HPO}_{4}\right)$, magnesium chloride $\left(\mathrm{MgCl}_{2}\right)$, benzonase nuclease, dithiothreitol (DTT), formic acid, ammonia solution, acetonitrile (ACN), and ethanol were obtained from Merck (Darmstadt, Germany). RapiGest ${ }^{\mathrm{TM}}$ SF was obtained from Waters Corporation (Manchester, UK). Titanium dioxide $\left(\mathrm{TiO}_{2}\right)$ resins were obtained from GL Sciences Inc. (Tokyo, Japan).

\subsubsection{U1 snRNPs Purification}

U1 snRNPs were purified from HeLa nuclear extract with mAb H2O by Prof. Reinhard Lührmann's Laboratory [93] and fractionated by glycerol gradient centrifugation by Monika Raabe [94]. The resulting complex was treated RNase (T1, A, Benzonase) at $37{ }^{\circ} \mathrm{C}$ for 2 hours, and subsequently at $52{ }^{\circ} \mathrm{C}$ for 2 hours. After, the proteins were isolated either by ethanol or acetone precipitation. The resulting pellet was resuspensed by $0.5 \%$ RapiGest $^{\mathrm{TM}}$ SF in 50 mM HEPES at $\mathrm{pH} 7.5$, and diluted to $0.1 \%$ RapiGest $^{\mathrm{TM}}$ SF with $50 \mathrm{mM}$ HEPES at $\mathrm{pH} 7.5$ for CPP.

\subsubsection{Ethanol Precipitation}

U1 snRNPs were precipitated by adding 3 volumes of ethanol and 1/10 volume of $3 \mathrm{M}$ sodium acetate, $\mathrm{pH}$ 5.3. The mixture was vortexed, incubated at $-20{ }^{\circ} \mathrm{C}$ for 2 hours and then centrifuged $17000 \mathrm{~g}$ at $4{ }^{\circ} \mathrm{C}$ for $30 \mathrm{~min}$. The supernatant was removed and the pellet was washed with $500 \mu \mathrm{l}$ of $80 \%$ ethanol and centrifuged as above. Discarded supernatant, the pellet was evaporated with a SpeedVac.

\subsubsection{Acetone Precipitation}

U1 snRNPs were precipitated by adding 4 volumes of acetone at $-20{ }^{\circ} \mathrm{C}$ for 2 hours and then centrifuged $17000 \mathrm{~g}$ at $4{ }^{\circ} \mathrm{C}$ for $30 \mathrm{~min}$. The supernatant was removed and the pellet was washed with $500 \mu \mathrm{l}$ of $80 \%$ acetone and centrifuged as above. Discarded supernatant, the pellet was evaporated with a SpeedVac.

\subsubsection{Optimized Calcium Phosphate Precipitation}


Phosphoproteins were precipitated by adding one-tenth volumes of $100 \mathrm{mM} \mathrm{CaCl}_{2}$ in $50 \mathrm{mM}$ HEPES, pH 7.5 for 10 min and then adding another one-tenth volumes of $50 \mathrm{mM} \mathrm{NaHPO}_{4}$ in $50 \mathrm{mM}$ HEPES, pH 7.5 for $10 \mathrm{~min}$ to form co-precipitation. The solution was centrifuged at $17000 \times \mathrm{g}$ for $10 \mathrm{~min}$, removed the supernatant to eppendorf, and $10 \mu \mathrm{l}$ of $50 \mathrm{mM} \mathrm{CaCl}$ in 50 mM HEPES, pH 7.5 was applied to wash the pellet. After centrifugation as described above, the washing solution was collected to the same eppendorf, and the resulting pellet was dissolved in $5 \mu$ of $1 \%$ RapiGest $^{\text {TM }} \mathrm{SF}$ in $25 \mathrm{mM}$ ammonium bicarbonate, $\mathrm{pH} 8.5$, sonicated for $15 \mathrm{~min}$, and subsequently analyzed by 1-D SDS-PAGE or performed in solution enzymatic digestion.

\subsubsection{In-Solution Digestion}

The protein solution was reduced with $5 \mu \mathrm{l}$ of $10 \mathrm{mM} \mathrm{DTT}$ at $37{ }^{\circ} \mathrm{C}$ for 1 hour, alkylated with 10 $\mu \mathrm{l}$ of $20 \mathrm{mM} \mathrm{CAA}$ at $37{ }^{\circ} \mathrm{C}$ for 1 hour, diluted to $0.1 \%$ RapiGest $^{\mathrm{TM}}$ SF with $30 \mu \mathrm{l}$ of $25 \mathrm{mM}$ ammonium bicarbonate, and subsequently digested with either trypsin or Asp-N/Lys-C at 37 ${ }^{\circ} \mathrm{C}$, overnight (the enzyme to substrate ratio is $1: 20$ ). The peptides were acidified with $50 \mu \mathrm{l}$ of 5 $\%$ TFA at $37{ }^{\circ} \mathrm{C}$ for 2 hours and centrifuged at $17000 \mathrm{~g}$ for $10 \mathrm{~min}$. Afterward, the supernatant was transferred to another eppendorf and dried on a SpeedVac for further analysis.

\subsection{7 $\mathrm{TiO}_{2}$ Enrichment Procedure}

Phosphopeptides were enriched by $\mathrm{TiO}_{2}$ as described (chapter 2). In brief, aliquots of peptides were dissolved with $20 \mu \mathrm{l}$ of $200 \mathrm{mg} \mathrm{DHB}$ in $1 \mathrm{ml}$ of $80 \%$ ACN, $5 \%$ TFA, and then loaded onto $\mathrm{TiO}_{2}$ column. The column was washed 3 times with $20 \mu \mathrm{l}$ of $200 \mathrm{mg}$ DHB in $1 \mathrm{ml}$ of $80 \% \mathrm{ACN}$, $5 \%$ TFA and 5 times with $20 \mu \mathrm{l} 80 \%$ ACN, $5 \%$ TFA. Bound peptides were eluted 3 times with $20 \mu \mathrm{l}$ of $0.3 \mathrm{~N} \mathrm{NH}_{4} \mathrm{OH}, \mathrm{pH} \geq 10.5$ and subsequently evaporated with a SpeedVac for further MS analysis.

\subsubsection{Mass Spectrometry Analysis}

For MALDI-MS analysis, the resulting peptides were mixed with a $10 \mathrm{mg} / \mathrm{ml}$ matrix solution of DHB in $70 \%$ ACN with $0.1 \%$ trifluoroacetic acid, and then spotted onto MALDI target plate. Subsequently, MALDI-MS detection was performed on a 4800 MALDI ToF/ToF mass spectrometry (Applied Biosystems/MDS Sciex) equipped with an Nd:YAG laser (355 nm wavelength, and $200 \mathrm{~Hz}$ repetition rate). 1000 shots were accumulated in positive ion mode MS. For the nanoLC-ESI MS/MS analysis, the resulting peptides were first loaded at a flow rate of $10 \mu \mathrm{l} / \mathrm{min}$ onto an in-house packed $\mathrm{C} 18$ trap column $(1.5 \mathrm{~cm}, 360 \mu \mathrm{m}$ o.d., $150 \mu \mathrm{m}$ i.d., Reprosil-Pur $120 \AA, 5 \mu \mathrm{m}, \mathrm{C} 18-\mathrm{AQ}$, Dr. Maisch $\mathrm{GmbH}$, Germany). The retained peptides were then eluted and separated on an analytical $C 18$ capillary column $(15 \mathrm{~cm}, 360 \mu$ m o.d., $75 \mu \mathrm{m}$ i.d., Reprosil-Pur $120 \AA, 5 \mu \mathrm{m}, \mathrm{C} 18-\mathrm{AQ}$, Dr. Maisch $\mathrm{GmbH}$, Germany) at a flow rate of 300 
$\mathrm{nL} / \mathrm{min}$, with a gradient from 7.5 to $37.5 \% \mathrm{ACN}$ in $0.1 \%$ formic acid for $60 \mathrm{~min}$ using an Agilent 1100 nano-flow LC system (Agilent Technologies, Palo Alto, CA), coupling with LTQ-Orbitrap XL hybrid mass spectrometer (Thermo Electron, Bremen, Germany). The LTQ-Orbitrap was operated in the data-dependant mode. Briefly, survey full scan MS spectra were acquired in the Orbitrap (m/z 350-1600) with the resolution set to 30,000 at $\mathrm{m} / \mathrm{z} 400$ and automatic gain control (AGC) target at $10^{6}$. The five most intense ions were sequentially isolated for CID MS/MS fragmentation and detection in the linear ion trap with previously selected ions dynamically excluded for 90 second. lons with singly and unrecognized charge state were also excluded. To improve the fragmentation spectra of the phosphopeptides, "multistage activation" corresponding to a neutral loss of phosphoric acid from doubly- and triply charged precursor ion was enabled in all MS/MS events [132].

\subsubsection{MASCOT Database Searching}

All spectra were searched MASCOT server v2.2.06 with the decoy database searching option (for assessing false positive rate) against the International Protein Index (IPI) human database (v3.62, 83947 protein sequences) with criteria-peptide mass tolerance, 7 ppm; MS/MS ion mass tolerance, $0.5 \mathrm{Da}$; allow up to three missed cleavage; variable modifications considered were phosphorylation of serine, threonine and tyrosine, methionine oxidation and cysteine carboxyamidomethylation. Filtering criteria were established based on decoy database search results to provide a $<1 \%$ false positive rate (FPR) for overall peptide identifications. All phosphorylated sited were examined manually by the presence of a $69 \mathrm{Da}$ between fragment ions for phosphoserine and an $83 \mathrm{Da}$ for phosphothreonine.

\subsection{Result and Discussion}

\subsubsection{Comparison of Efficiency and Specificity among Different Alkaline Earth Metal}

The identification of low abundant phosphoproteins and the subsequent identification of the actual phosphorylation site(s) typically necessitate their enrichment prior to MS analysis. Our goal was to develop a straightforward, sensitive, and MS compatible method for the enrichment of intact phosphoproteins. The basic concept originated from the lowered solubility product of phosphate group when formed a complex with divalent alkaline earth metal cations in aqueous solution.

To demonstrate our speculation, we decided to first carry out preliminary experiment with the same concentration ratio of $\mathrm{MgCl}_{2} / \mathrm{Na}_{2} \mathrm{HPO}_{4}$ and $\mathrm{CaCl}_{2} / \mathrm{Na}_{2} \mathrm{HPO}_{4}$ (2:1) for segregation of phosphoproteins. In this study, beta-casein was used as a model phosphoprotein due to its multiple phosphorylation sites $[71,75,133] .10 \mu$ of standard protein solution, consisting of a 
total amount of $5 \mu \mathrm{g}$ each of beta-casein, BSA and myoglobin in $50 \mathrm{mM}$ HEPES buffer solution $(\mathrm{pH}$ 7.5), was utilized for examine the efficiency of phosphoprotein enrichment. One-tenth volume of $50,100,250$ and $500 \mathrm{mM} \mathrm{MgCl}_{2}$ or $\mathrm{CaCl}_{2}$ solution was added to sample solution for $10 \mathrm{~min}$, and followed additional one-tenth volume of 25, 50, 125 and $250 \mathrm{mM} \mathrm{NaHPO}{ }_{4}$ was added for another $10 \mathrm{~min}$ to form co-precipitation of phosphoprotein, respectively. The resulting protein pellet could be observed after high speed centrifugation (17000 x g for 10 min). After, the supernatant and pellet were analyzed by 1-D SDS-PAGE to visualize their effects shown in Figure 3.1.
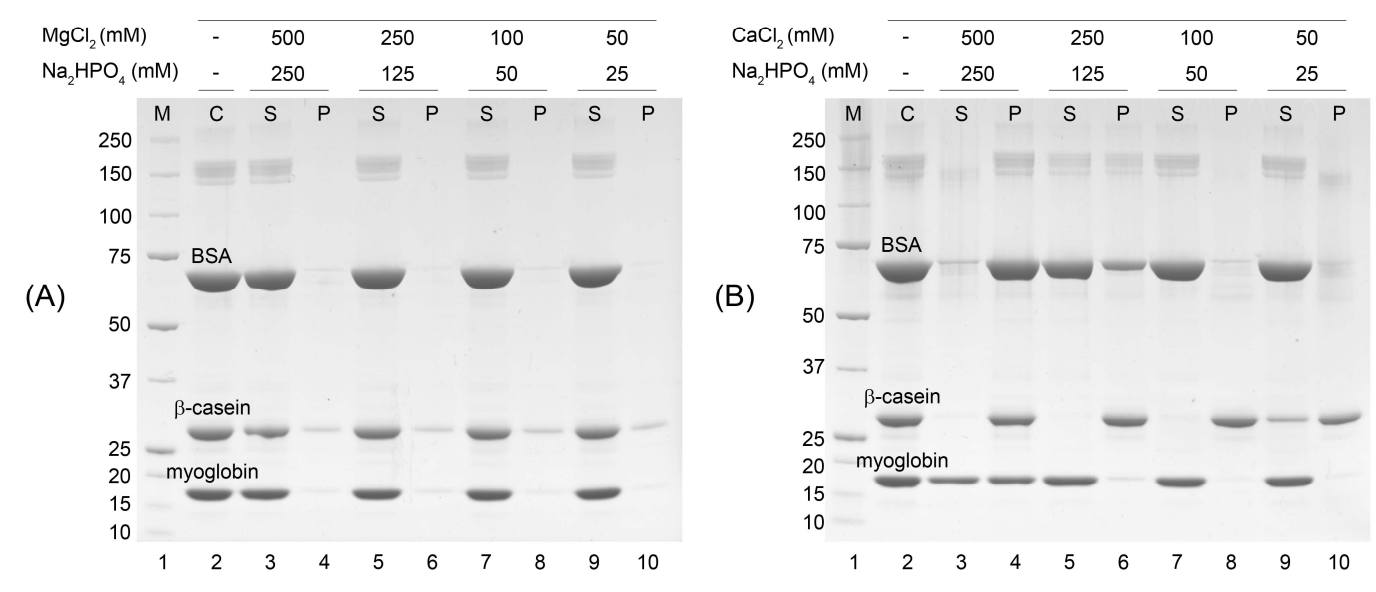

Figure 3.1. SDS-PAGE (4-12 \%, Coomassie blue staining) analysis of standard proteins precipitated by alkaline earth metal. (A) Magnesium phosphate precipitation, 5 $\mu \mathrm{g}$ each of beta-casein, BSA and myoglobin were used to precipitate model phosphoprotein, beta-casein, by adding $\mathrm{MgCl}_{2} / \mathrm{Na}_{2} \mathrm{HPO}_{4}$ in concentration order from 500 $\mathrm{mM} / 250 \mathrm{mM}$ (Lane 3 and 4), $250 \mathrm{mM} / 125 \mathrm{mM}$ (Lane 5 and 6), $100 \mathrm{mM} / 50 \mathrm{mM}$ (Lane 7 and 8) to $50 \mathrm{mM} / 25 \mathrm{mM}$ (Lane 9 and 10), respectively. (B) Calcium phosphate precipitation (CPP), instead of $\mathrm{MgCl}_{2} / \mathrm{Na}_{2} \mathrm{HPO}_{4}, \mathrm{CaCl}_{2} / \mathrm{Na}_{2} \mathrm{HPO}_{4}$ was used to precipitate beta-casein with the same concentration. M: Protein marker. C: Control, standard proteins. S: Supernatant. P: Pellet.

By using $\mathrm{MgCl}_{2} / \mathrm{Na}_{2} \mathrm{HPO}_{4}$ precipitation of phosphorylation (Figure 3.1A), most of the proteins, beta-casein, BSA and myoglobin, were present in the supernatant. Only a few amount of beta-casein could be effectively separated into pellet fraction and the loss of beta-casein took place. Even the concentration of $\mathrm{MgCl}_{2} / \mathrm{Na}_{2} \mathrm{HPO}_{4}$ increased up to $500 \mathrm{mM} / 250 \mathrm{mM}$, the majority of beta-casein still retained in supernatant (Lane 3 and 4). With respect to $\mathrm{MgCl}_{2} /$ $\mathrm{Na}_{2} \mathrm{HPO}_{4}$ precipitation, $\mathrm{CaCl}_{2} / \mathrm{Na}_{2} \mathrm{HPO}_{4}$ seemed to be more efficiency to isolate beta-casein from BSA and myoglobin (Figure 3.1B). As $50 \mathrm{mM} / 25 \mathrm{mM}$ of $\mathrm{CaCl}_{2} / \mathrm{Na}_{2} \mathrm{HPO}_{4}$ was used to precipitate, more than half of beta-casein was precipitated into pellet fraction (Lane 10). But, it 
still remained a smaller part of beta-casein in the supernatant (Lane 9). Until the concentration of $\mathrm{CaCl}_{2} / \mathrm{Na}_{2} \mathrm{HPO}_{4}$ raised to $100 \mathrm{mM} / 50 \mathrm{mM}$, the majority of beta-casein was segregated into pellet fraction (Lane 8), and non-phosphorylated proteins still retained in the supernatant (Lane 7). However, while the concentration of $\mathrm{CaCl}_{2} / \mathrm{Na}_{2} \mathrm{HPO}_{4}$ increased up to $250 \mathrm{mM} / 125$ $\mathrm{mM}, \mathrm{BSA}$ started to form precipitation (Lane 6). Under high concentration of $\mathrm{CaCl}_{2} / \mathrm{Na}_{2} \mathrm{HPO}_{4}$ (500 mM / $250 \mathrm{mM}$ ), almost all BSA and half of myoglobin were present in the pellet fraction (Lane 4). Hence, we found out optimized concentration of $\mathrm{CaCl}_{2} / \mathrm{Na}_{2} \mathrm{HPO}_{4}$ was $100 \mathrm{mM} / 50$ mM. According to these results, we can draw a short conclusion that $\mathrm{CaCl}_{2} / \mathrm{Na}_{2} \mathrm{HPO}_{4}$ has a strong ability to efficiently isolate phosphoproteins.

\subsubsection{Optimal Condition for Phosphoprotein Precipitation}

We further investigated whether the results could be improved by altering $\mathrm{Na}_{2} \mathrm{HPO}_{4}$ concentration. The standard proteins were performed CCP by using $100 \mathrm{mM} \mathrm{CaCl}_{2}$ in combined with $250,125,50$ and $25 \mathrm{mM} \mathrm{Na}_{2} \mathrm{HPO}_{4}$, respectively. The resulting pellet and supernatant were analyzed by SDS-PAGE shown in Figure 3.2. Under $250 \mathrm{mM} \mathrm{Na} \mathrm{HPO}_{4}$ condition, non-phosphorylated proteins could be formed precipitation (Lane 2 and 3). No significant difference was observed by using 50 or $125 \mathrm{mM} \mathrm{Na}_{2} \mathrm{HPO}_{4}$ (Lane 4 to 7). All beta-casein could be fractionated into pellet fraction and non-phosphorylated proteins still remained in the supernatant. Under $25 \mathrm{mM} \mathrm{Na} \mathrm{HPO}_{4}$ condition, the result indicated this concentration did not provided sufficient phosphate anion to co-precipitate beta-casein with $100 \mathrm{mM} \mathrm{CaCl}_{2}$, leading to a smaller part of beta-casein in the supernatant (Lane 8 and 9). The result reveals that beta-casein only be isolated within 50 to $125 \mathrm{mM} \mathrm{Na}_{2} \mathrm{HPO}_{4}$.

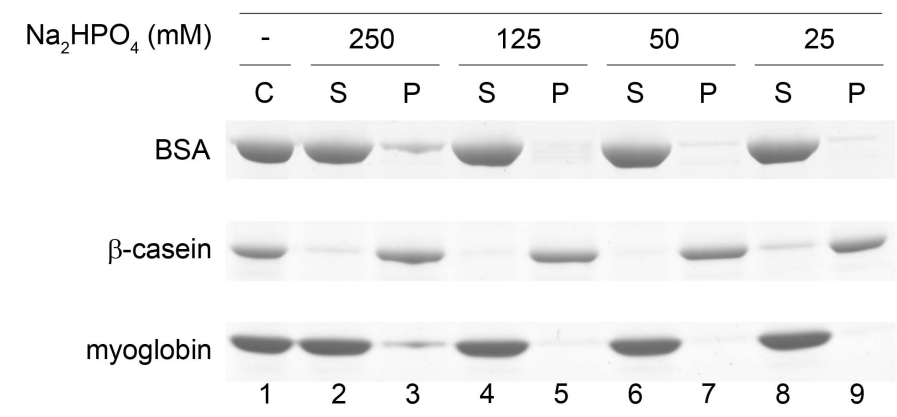

Figure 3.2. SDS-PAGE analysis of standard protein under different concentration of $\mathrm{Na}_{2} \mathrm{HPO}_{4}$ to optimize CPP procedure. One-tenth volume of $100 \mathrm{mM} \mathrm{CaCl} 2$ was added to $5 \mu \mathrm{g}$ each of beta-casein, BSA and myoglobin solution, and followed additional one-tenth volume of $250,125,50$ and $25 \mathrm{mM} \mathrm{Na}_{2} \mathrm{HPO}_{4}$ to form co-precipitation, respectively. C: Control, standard proteins. S: Supernatant. P: Pellet. 
In this context, the selective isolation of phosphoprotein would necessitate the incorporation of not only $\mathrm{CaCl}_{2} / \mathrm{Na}_{2} \mathrm{HPO}_{4}$ concentration but also a wash buffer solution that provides sufficient strength to compete off all other non-phosphorylated proteins. This would leave almost all phosphoproteins in pellet, which could then be directly applied to digest for MS and MS/MS analysis. The CPP resulting pellet was washed $10 \mu \mathrm{l}$ of $50 \mathrm{mM} \mathrm{CaCl}$ or $25 \mathrm{mM} \mathrm{Na}_{2} \mathrm{HPO}_{4}$ solution either one time or two times shown in Figure 3.3A. Comparative analyses showed that $50 \mathrm{mM} \mathrm{CaCl} 2$ performed significantly better than $25 \mathrm{mM} \mathrm{Na}_{2} \mathrm{HPO}_{4}$ for retaining beta-casein and washing off the non-phosphorylated proteins (Lane 2 to 5). The obtained pellet of beta-casein could be displaced with phosphate anion, leading to low yields (Lane 6 to 9). In addition, one time wash of $\mathrm{CaCl}_{2}$ was sufficient. The signal of beta-casein was absent in the supernatant which is indicative of its minimal loss from at this stage (Lane 2 and 3). We also tested the effect of $\mathrm{pH}$ value in wash buffer solution, as shown in Figure 3.3B. The CPP resulting pellet was washed with $25 \mathrm{mM} \mathrm{CaCl}_{2}$ in $50 \mathrm{mM} \mathrm{HEPES}, \mathrm{pH} 7.5 ; 0.1 \%$ formic acid (FA), $\mathrm{pH} 3 ; 0.1 \%$ trifluoroacetic acid (TFA), $\mathrm{pH} 2$ or $0.3 \mathrm{~N}$ ammonium hydroxide $\left(\mathrm{NH}_{4} \mathrm{OH}\right), \mathrm{pH}$ 10.5 , respectively. A low abundance signal of beta-casein could be detected in the supernatant with respect to FA, TFA and $\mathrm{NH}_{4} \mathrm{OH}$ washing buffer (Lane 4, 6 and 8). Our result suggests that it is better to use $50 \mathrm{mM} \mathrm{HEPES} \mathrm{pH} 7.5$ to wash off non-phosphorylated proteins, which is also compatible for further enzymatic digestion and MS analysis.

(A)

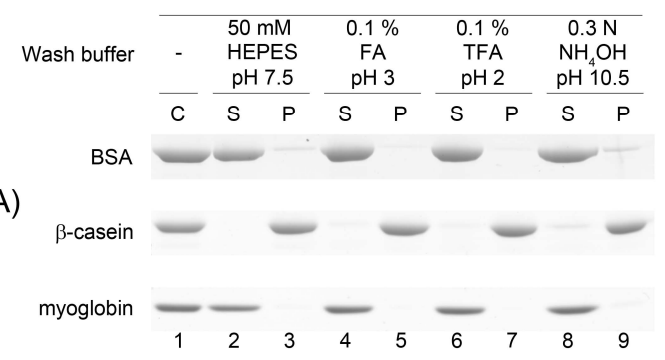

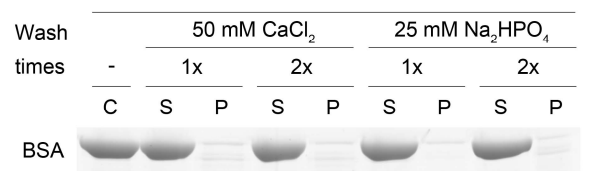

(B)

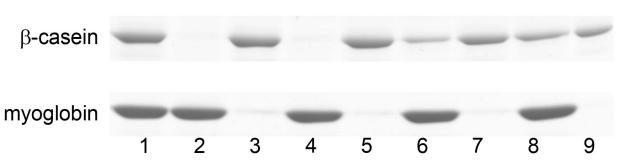

Figure 3.3. SDS-PAGE analysis of standard protein by using different wash conditions to optimize CPP procedure. (A) After CPP $\left(\mathrm{CaCl}_{2} / \mathrm{Na}_{2} \mathrm{HPO}_{4}, 100 \mathrm{mM} / 50\right.$ $\mathrm{mM}$ ), the resulting protein pellet was washed with $10 \mu \mathrm{l}$ of $50 \mathrm{mM} \mathrm{CaCl}_{2}$ or $25 \mathrm{mM}$ $\mathrm{Na}_{2} \mathrm{HPO}_{4}$ either one time or two times. (B) After CPP $\left(\mathrm{CaCl}_{2} / \mathrm{Na}_{2} \mathrm{HPO}_{4}, 100 \mathrm{mM} / 50 \mathrm{mM}\right)$, the protein pellet was washed with $50 \mathrm{mM} \mathrm{CaCl}_{2}$ in $50 \mathrm{mM}$ HEPES buffer, $0.1 \%$ formic acid (FA) , $0.1 \%$ trifluoroacetic acid (TFA), or $0.3 \mathrm{~N}$ ammonium hydroxide $\left(\mathrm{NH}_{4} \mathrm{OH}\right)$, respectively. C: Control, standard proteins. S: Supernatant. P: Pellet.

Here, we propose two possibilities that allow the formation of co-precipitation of beta-casein. One is due to the phosphate groups on beta-casein that can interact with calcium chloride and disodium hydrogen phosphate to form insoluble calcium-phosphate-protein complex. The other is owing to the structure property of beta-casein. To clarify the two possibilities, the 
standard protein mixture was first dephosphorylated by alkaline phosphatase and performed the optimized CPP procedure shown in Figure 3.4. The result indicated beta-casein was no longer precipitating while phosphate groups on beta-casein were removed by alkaline phosphatase (Lane 5 and 6 ). This result indeed confirms our speculation that phosphoprotein can be isolated by our CPP based on their solubility.

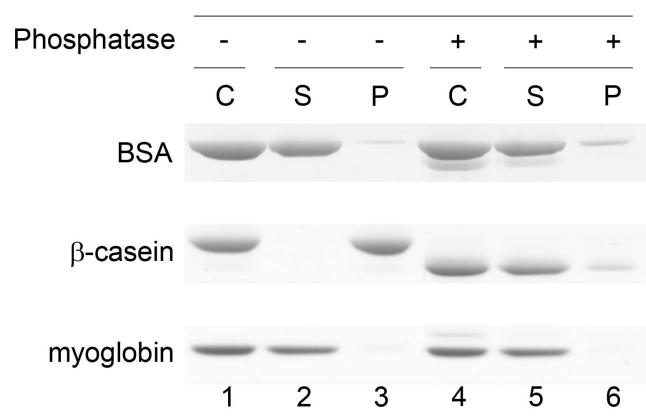

Figure 3.4. Proof of the CCP procedure by virtue of the formation of insoluble calcium-phosphate-protein complex. The standard proteins, $5 \mu \mathrm{g}$ of each beta-casein, BSA and myoglobin, were performed with optimized CPP procedure to segregate model phosphoprotein, beta-casein, shown in Lane 1, 2 and 3. Before CPP, the standard proteins were treated with alkaline phosphatase, shown in Lane 4, 5 and 6. C: Control, standard proteins. S: Supernatant. P: Pellet.

\subsubsection{Effect of Different Denatured Reagents}

For shotgun proteomic analysis, proteins must be soluble and denature in an aqueous solution for enzymatic digestion. The most frequent method of dissolving proteins is the use of ionic detergents such as sodium dodecyl sulphate (SDS). To test whether our CPP method is compatible to SDS, $5 \mu \mathrm{g}$ each of beta-casein, BSA and myoglobin was dissolved with $0.5,0.1$, 0.05 or $0.01 \%$ SDS in $50 \mathrm{mM}$ HEPES ( $\mathrm{pH} 7.5$ ) buffer solution, respectively, and isolation of beta-casein with optimized CPP procedure was followed, as shown in Figure 3.5.

The majority of beta-casein was preferentially present in the supernatant under $0.5 \%$ SDS condition (Lane 3). Although beta-casein could form precipitation under $0.1 \%$ and $0.05 \%$ SDS conditions, a smaller proportion of BSA and myoglobin was also observed in the pellet fraction, which might due to the formation of protein micelles with SDS reagent (Lane 6 and 8). In the presence of $0.01 \%$ SDS, beta-casein was segregated again, inferring the concentration of SDS had to reduce less than $0.01 \%$ SDS in sample solution prior to CPP enrichment (Lane 9 and 10). The result indicated that the maximum concentration of SDS in sample solution had to be diluted less than $0.01 \%$ before the execution of CPP process. 


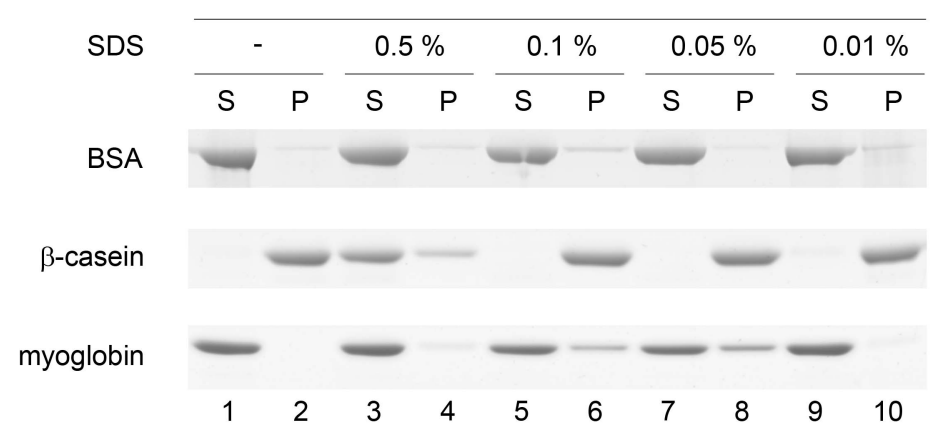

Figure 3.5. SDS-PAGE analysis of the influence of SDS detergent on our CPP method. $5 \mu \mathrm{g}$ each of beta-casein, BSA and myoglobin were dissolved in 0.5, 0.1, 0.05, and $0.01 \%$ SDS, respectively, and phosphoprotein was subsequently isolated by optimized CPP procedure.

We also tested another strong protein denaturant, urea, which unfold the protein and is widely used to dissolve sample in proteomic research [99, 134-136]. The same amount of standard proteins was dissolved with 0.6, 0.4, 0.2 and $0.1 \mathrm{~N}$ urea in $50 \mathrm{mM}$ HEPES ( $\mathrm{pH} 7.5$ ) buffer solution, respectively, and then precipitated by optimized CPP procedure shown in Figure 3.6. Although the majority of non-phosphorylated proteins were remained in the supernatant, a trace proportion of base-casein was observed in the pellet fraction under $0.6,0.4$ and $0.2 \mathrm{~N}$ urea condition (Lane 3, 5 and 7). Until the concentration of urea was decreased to $0.1 \mathrm{~N}$, the isolation of beta-casein by CPP was reconstructed (Lane 9 and 10). The result indicated that the maximum concentration of urea in sample solution should be diluted less than $0.1 \mathrm{~N}$ before the execution of CPP.

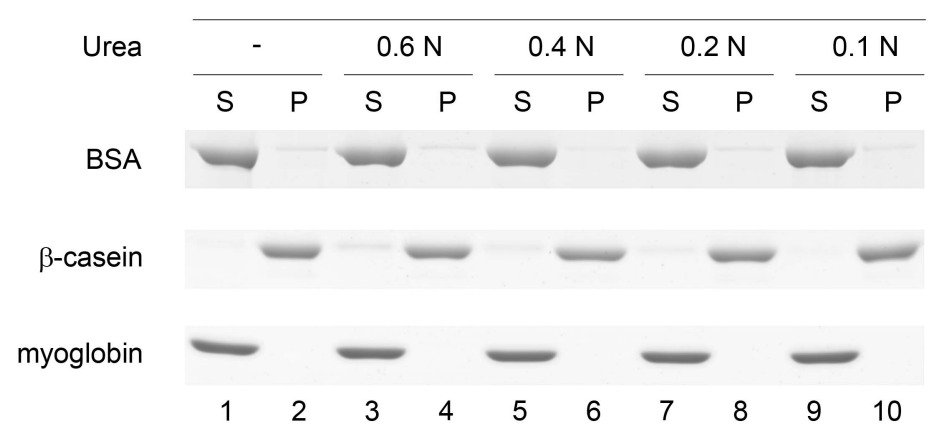

Figure 3.6. SDS-PAGE analysis of the influence of denature reagent, urea, on our CPP method. $5 \mu \mathrm{g}$ each of beta-casein, BSA and myoglobin were dissolved with 0.6, 0.4, 0.2 and $0.1 \mathrm{~N}$ urea, respectively, and subsequently isolated phosphoprotein by optimized CPP procedure.

Our final goal is to develop a straightforward and reliable method that can be applied to global 
proteome study. Not only pellet but also supernatant had to be analyzed. Notwithstanding SDS and urea under $0.01 \%$ and $0.1 \mathrm{~N}$, respectively, have a good efficiency for phosphoprotein enrichment, both reagents have shortcomings in shotgun proteomic method based on in-solution enzymatic digestion of proteins prior to MS analysis. The SDS is trapped on the reverse phase column and forms a cluster of ions during ESI ion source inferring peptide detection in MS. The urea caused carbamylation at $\mathrm{N}$-terminus of peptide residue or lysine residue [104-106], increasing the complexity of sample. Thus, we have to remove SDS and urea by running SDS-PAGE or other chromatographic technology prior to MS detection.

For this reason, we further tested a MS compatible denatured surfactant, RapiGest ${ }^{\mathrm{TM}} \mathrm{SF}$, an acid-cleavable anionic detergent that is used to enhance enzymatic digestion of proteins under $0.1 \%$ condition, and can be removed by high speed centrifugation [107]. $5 \mu \mathrm{g}$ each of standard proteins were dissolved with $0.5,0.1,0.05$ and $0.01 \%$ RapiGest $^{\mathrm{TM}} \mathrm{SF}$ in $50 \mathrm{mM}$ HEPES buffer solution, respectively, and subsequently precipitated by optimized CCP procedure in Figure 3.7.

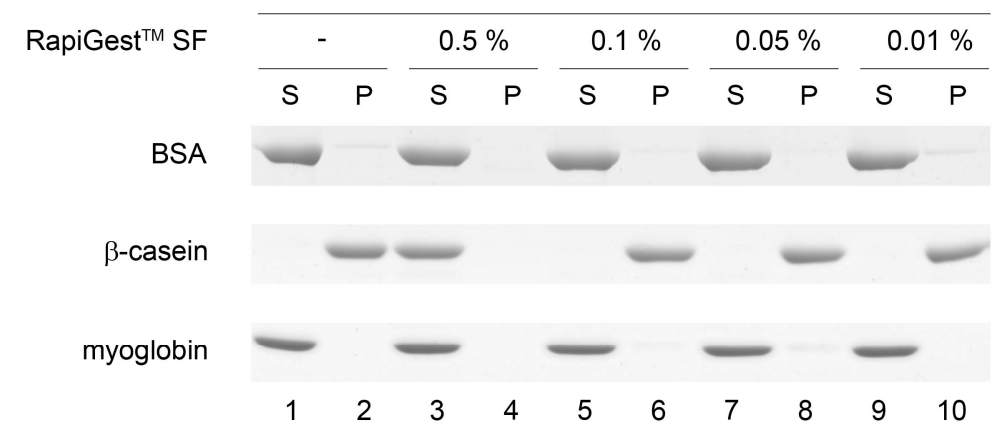

Figure 3.7. SDS-PAGE analysis of the influence of RapiGest ${ }^{\mathrm{TM}} \mathrm{SF}$ on our CPP method. $5 \mu \mathrm{g}$ each of beta-casein, BSA and myoglobin were dissolved with $0.6,0.4,0.2$ and $0.1 \mathrm{~N}$ urea, respectively, and subsequently isolated phosphoprotein by optimized CPP procedure.

Under $0.5 \%$ RapiGest $^{\mathrm{TM}}$ SF condition, all these three standard proteins were remained in the supernatant during the CPP process (Lane 3 and 4). Under 0.1, 0.05 and $0.01 \%$ RapiGest $^{\mathrm{TM}}$ SF conditions, the majority of beta-casein was present only in the pellet fraction (Lane 6, 8 and 10), and BSA and myoglobin were still retained in the supernatant (Lane 5, 7 and 9). The result indicated our CPP method could tolerate upto $0.1 \%$ RapiGest $^{\mathrm{TM}}$ SF that was susceptible to enzymatic cleavage without inhibiting enzyme activity. It is worth to note that Xumin et al. used calcium phosphate to precipitate phosphopeptides, and the resulting pellet was dissolved in 5 $\%$ formic acid prior to MS analysis [69]. However, $5 \%$ formic acid inhibits most of in-solution 
enzymatic digestion such as trypsin, Asn-N, Glu-C and so on. Taken together, the advantages of using RapiGest TM SF include its ability to dissolve the protein complex pellet under $0.5 \%$ condition, compatible for CPP under $0.1 \%$ condition, improving enzymatic digestion, and acid-labile that can be easily removed by high speed centrifugation without the interference of the followed MS analysis.

\subsubsection{Examine the Power of our Phosphoprotein Isolation Method}

To assess the efficiency and specificity of our CPP procedure for enrichment of phosphoprotein, different amounts of beta-casein $(5,3.75,2.5,1.25,0.5$ and $0.25 \mu \mathrm{g}$, respectively) containing $5 \mu \mathrm{g}$ BSA and $5 \mu \mathrm{g}$ myoglobin were prepared for examining the power of our CPP procedure shown in Figure 3.8.

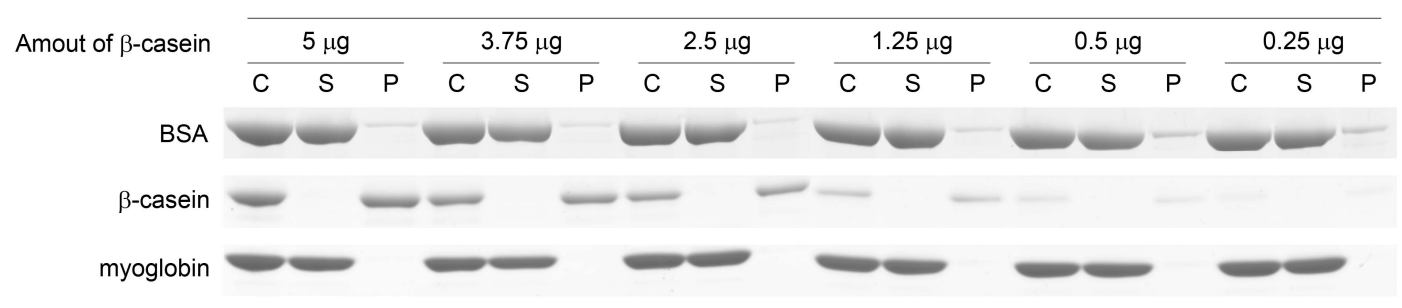

Figure 3.8. The efficiency and specificity of our CPP procedure. Different amount of beta-casein from $5,3.75,2.5,1.25,0.5$ to $0.25 \mu \mathrm{g}$ containing of $5 \mu \mathrm{g} \mathrm{BSA}$ and $5 \mu \mathrm{g}$ myoglobin in solution was performed optimized CCP procedure, and subsequently analyzed by SDS-PAGE, respectively. C: Control, standard proteins. S: Supernatant. P: Pellet.

The result indicated that most of BSA and myoglobin were present in the supernatant. Beta-casein only remained in the pellet fraction, even in low amount of beta-casein $(0.25 \mu \mathrm{g}$, approximately $10 \mathrm{pmol}$, close to the limit of detection of Coomassie blue staining). However, a smaller proportion of BSA still retained in the pellet fraction, indicating that our CPP method is still not sufficient for isolating 20 fold substoichiometric levels of phosphoprotein for subsequently unseparated enzymatic digestion of protein analysis by MALDI-MS. An additional enrichment for phosphopeptides is necessitated to increase the specificity by reducing the amount of non-phosphopeptides. So, we coupled our CPP method with in-house $\mathrm{TiO}_{2}$ microspin column enrichment. The CPP resulting proteins, either pellet or supernatant, were digested with trypsin, enriched with in-house $\mathrm{TiO}_{2}$ microspin column and then spotted on MALDI target plate for MALDI-MS analysis. Figure 3.9.A showed MALDI-MS spectrum of tryptic peptides derived from $0.25 \mu \mathrm{g}$ beta-casein containing $5 \mu \mathrm{g}$ BSA and $5 \mu \mathrm{g}$ myoglobin. However, signals of phosphopeptides derived from beta-casein at $\mathrm{m} / \mathrm{z} 2061.9$ and 3122.3 were not detected in the mass spectrum. That is because the signal corresponding to 
phosphopeptides which is readily detectable in MS is suppressed in the company of other more readily ionized non-phosphorylated peptides of BSA and myoglobin. Even these peptides were enriched with $\mathrm{TiO}_{2}$, only one phosphopeptide of beta-casein at m/z 2061.9 was observed in the mass spectrum shown in Figure 3.9D.

(A)

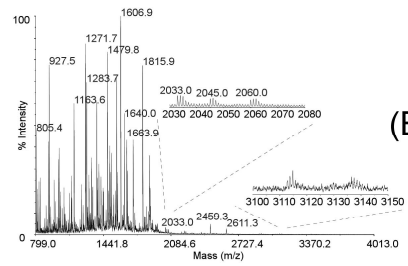

(B)

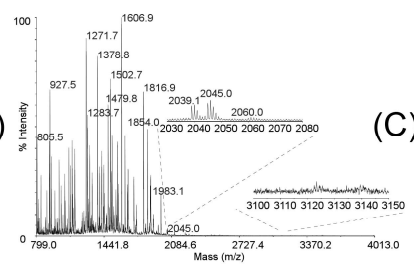

(C)

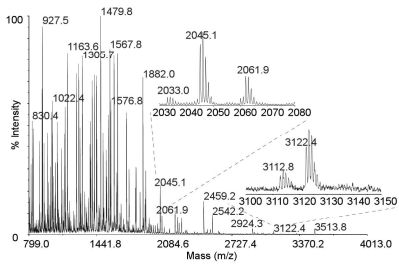

(D)
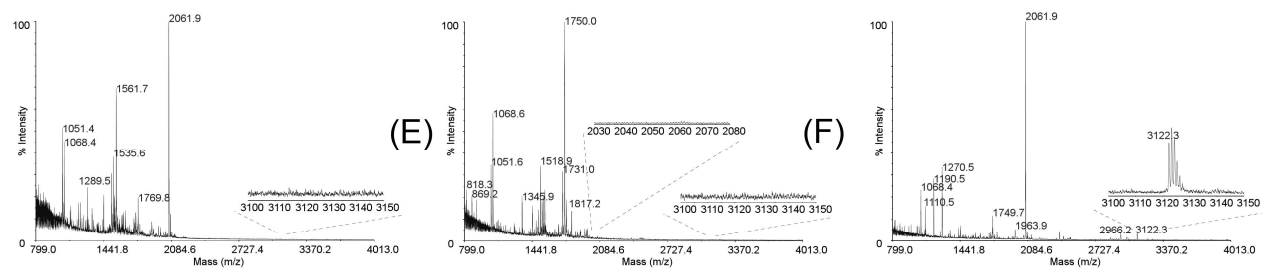

Figure 3.9. The identification of phosphopeptide using CPP method coupling with in-house $\mathrm{TiO}_{2}$ microspin column. MALDI mass spectrum of tryptic peptides derived from $0.25 \mu \mathrm{g}$ beta-casein containing of $5 \mu \mathrm{g}$ BSA and $5 \mu \mathrm{g}$ myoglobin in solution, one two-hundredth tryptic peptides were spotted on the MALDI target plate. (A) and (D) without CPP; $(B)$ and $(E)$ the CPP resulting supernatant; $(C)$ and $(F)$ the CPP resulting pellet. (D), $(E)$ and $(F)$ with an additional phosphopeptide enrichment with in-house $\mathrm{TiO}_{2}$ microspin column. Two signals of phosphopeptides derived from beta-casein at $\mathrm{m} / \mathrm{z} 2061.9$ and 3122.3 are FQpSEEQQQTEDELQDK and RELEELNVPGEIVEpSLpSpSpSEESITR, respectively.

Possible explanation of not detecting tetraphosphorylated peptide at $\mathrm{m} / \mathrm{z} 3122.3$ in the mass spectrum may due to large amount of non-phosphorylated peptides in solution which could inference $\mathrm{TiO}_{2}$ enrichment. After CPP, the spectrum obtained from tryptic peptides of supernatant (Figure 3.9B) was almost identical to that of the original sample (Figure 3.9A). All signals in the spectrum belonged to tryptic peptides of BSA and myoglobin. Even an additional $\mathrm{TiO}_{2}$ enrichment was performed, the signals corresponding to phosphopeptides of beta-casein at $\mathrm{m} / \mathrm{z} 2061.9$ and 3122.3 were still not detectable in the spectrum shown in Figure 3.9E, indicating that the loss of phosphoprotein is minimal during the entire CPP procedure. The CPP resulting pellet was redissolved in $0.5 \%$ RapiGest $^{\mathrm{TM}}$ SF buffer solution and then was diluted to $0.1 \%$ for trypsin digestion. The respective phosphopeptides for beta-casein, including the signals at m/z 2061.9 and 3122.3 which were not detected before were now observed shown in Figure 3.9C; however, the spectrum still accompanied with other signals of tryptic peptides 
derived from BSA and myoglobin. In combination with $\mathrm{TiO}_{2}$ enrichment, the majority portion of non-phosphorylated peptides were removed by $\mathrm{TiO}_{2}$ and phosphopeptide signals derived from beta-casein at m/z 2061.9 and 3122.3 dominated the spectrum shown in Figure 3.9F. This result indeed indicated that our $\mathrm{CPP}$ and $\mathrm{TiO}_{2}$ were complementary methods for trace level phosphopeptides enrichment. Taking cues from previous findings, the CPP procedure can specifically and effectively concentrate phosphoproteins from the complex protein mixture and further $\mathrm{TiO}_{2}$ enrichment of phosphopeptides from CPP resulting pellet enable efficient recovery of phosphopeptides, even when substoichiometric levels of the non-phosphorylated protein is 20 folds higher than that of the phosphoprotein.

\subsubsection{Identification of Phosphorylation Sites from U1 Small Nuclear Ribonucleoproteins}

To investigate whether our CPP method in coupled with $\mathrm{TiO}_{2}$ enrichment was applicable to a very complicated biological sample for identification of phosphorylation site, the method was tested in one of our ongoing projects, the U1 small nuclear ribonucleoproteins (snRNPs). The snRNPs are the protein complexes combined with pre-mRNA to form a spliceosome. The pre-mRNA splicing can be regulated both positively and negatively by reversible protein phosphorylation. Figure 3.10 showed the SDS-PAGE analysis of $10 \mu \mathrm{g} U 1 \mathrm{snRNPs}$ fractioned by CPP method.

However, the pre-mRNA contains phosphate groups, and the strong protein-protein and protein-RNA interaction occur in U1 snRNPs, resulting in the co-precipitation of proteins which does not contain any phosphorylation shown in Lane 3 and 4 . To minimize the affection, $0.5 \%$ RapiGest TM SF was used to denature U1 snRNPs, and then diluted to $0.1 \%$ RapiGest ${ }^{\mathrm{TM}} \mathrm{SF}$ for the CPP of phosphoproteins (Lane 5 and 6). Nevertheless, the existing of pre-mRNA in solution also binds to $\mathrm{TiO}_{2}$ strongly, and interferes further phosphopeptides detection in MS analysis. Hence, prior to CPP enrichment, the U1 snRNPs was treated with ribonuclease (RNase T, T1 and benzonase) to cleave pre-RNA into smaller components, precipitated proteins with either acetone or ethanol to remove small RNA, and subsequently redissolved by RapiGest $^{\mathrm{TM}}$ SF shown in Lane 7 to 10 , respectively. The result indicated that acetone precipitation of U1 snRNPs had better proteins recovery than ethanol.

After RNase treatment, acetone precipitation, and CPP method, we kept the resulting supernatant and pellet in $0.1 \%$ RapiGest $^{\mathrm{TM}} \mathrm{SF}$, that was compatible for further in-solution enzymatic digestion in coupled with in-house $\mathrm{TiO}_{2}$ microspin column enrichment linked to MS analysis for identification of phosphorylated sites in U1 snRNPs. 


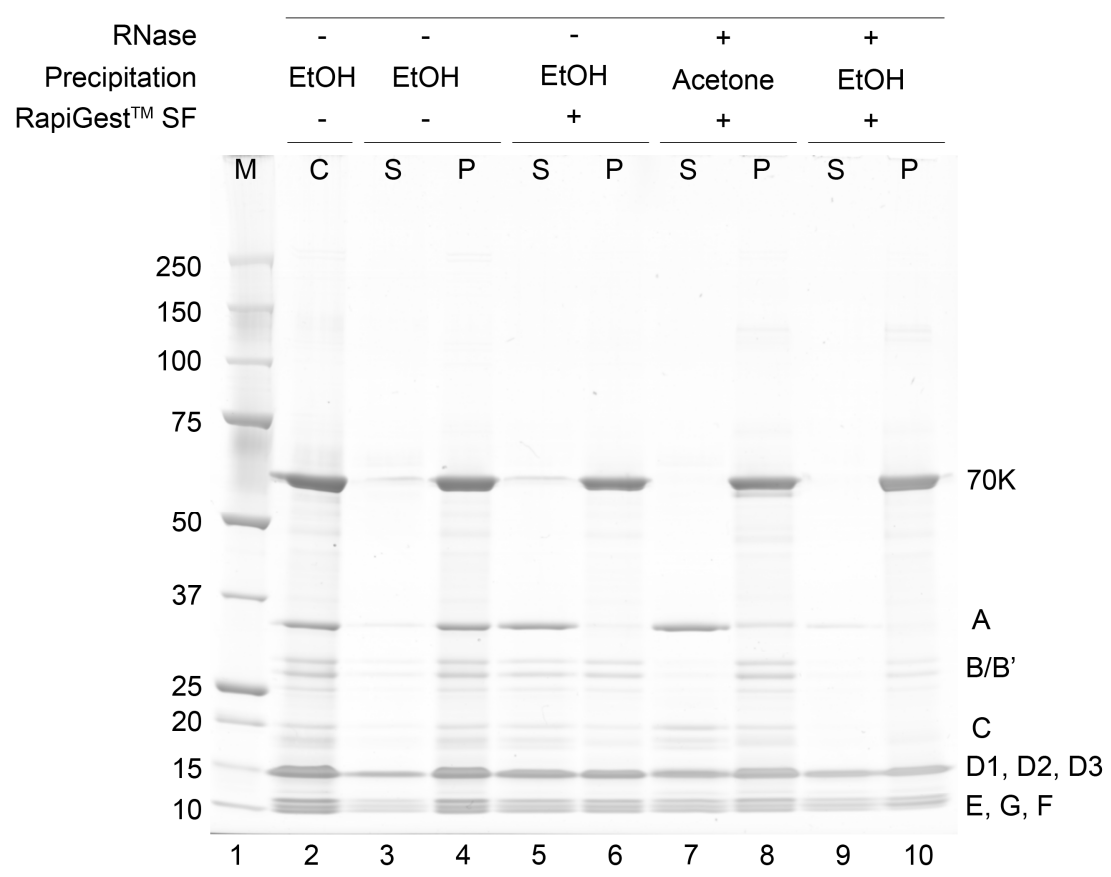

Figure 3.10. SDS-PAGE analysis of $10 \mu \mathrm{g}$ of $\mathrm{U} 1 \mathrm{snRNPs}$ extracted from HeLa cell. The extracted U1 snRNPs were purified by EtOH precipitation (Lane 2), dissolved in $50 \mathrm{mM}$ HEPES buffer, and subsequently performed with optimized CPP (Lane 3 and 4). Instead of HEPES buffer, RapiGest ${ }^{\mathrm{TM}}$ SF was used to dissolve U1 snRNPs (Lane 5 and 6). Before EtOH precipitation, the RNase was treated to cleavage pre-mRNA (Lane 9 and 10). Instead of EtOH precipitation, acetone was used to purify U1 snRNPs (Lane 7 and 8). M: Protein marker. C: Control. S: Supernatant. P: Pellet.

Table 1 provided a partial list of proteins found in the U1 snRNPs. Three U1-specific proteins (U1-70K, U1-A and U1-C) and Sm proteins (B'/B, D1, D2, D3, E, F and G) were identified with high MASCOT score on both fraction. However, the phosphopeptide was not detectable in the supernatant without $\mathrm{TiO}_{2}$ enrichment. Even these peptides were enriched with $\mathrm{TiO}_{2}$, only 4 phosphorylation sites were found. In comparison, a total of 192 unique phosphorylation sites corresponding to 45 distinct proteins had been identified from pellet fraction (Appendix 3 ). All phosphorylated sites were confirmed by manual validation. 59 phosphorylation sites identified in this study have not been reported previously, searching against Expasy Knowledgebase (http://us.expasy.org/) and PhosphoSite database (http://www.phosphosite.org/) [114]; these two databases that have collected comprehensive information on in vivo protein phosphorylation and references. 
Table 3.1. Partial list of phosphorylation sites identified from the leading proteins of U1 snRNPs by using CPP method in combination with in-house $\mathrm{TiO}_{2}$ microspin column enrichment. For all phosphorylation sites, see Appendix 3 . The underline indicates that the phosphorylation site was not identified previously.

\begin{tabular}{|c|c|c|c|c|c|c|c|}
\hline & & \multicolumn{3}{|c|}{ Supernatant } & \multicolumn{3}{|c|}{ Pellet } \\
\hline & & \multicolumn{2}{|c|}{$\mathrm{w} / \mathrm{o} \mathrm{\textrm {TiO } _ { 2 }}$} & \multirow{2}{*}{$\frac{\mathrm{TiO}_{2}}{p \text {-site }}$} & \multicolumn{2}{|c|}{ w/o $\mathrm{TiO}_{2}$} & \multirow{2}{*}{$\frac{\mathrm{TiO}_{2}}{\text { p-site }}$} \\
\hline & & $\begin{array}{c}\text { Mascot } \\
\text { score }\end{array}$ & $\mathrm{p}$-site & & $\begin{array}{l}\text { Mascot } \\
\text { score }\end{array}$ & p-site & \\
\hline \multirow[t]{8}{*}{ U1 specific } & $70 \mathrm{~K}$ & 618 & & S226; S266; & 825 & $\underline{\text { S117; }}$ & $\underline{\mathrm{Y} 38} ; \underline{\mathrm{Y} 112} ; \underline{\mathrm{S} 117}$ \\
\hline & & & & S268 & & S410 & Y126; Y206; $\underline{\mathrm{S} 211}$ \\
\hline & & & & & & & S226; S245; $\underline{\mathbf{S} 257} ;$ \\
\hline & & & & & & & $\underline{\mathrm{S} 259}$; S266; S268; \\
\hline & & & & & & & $\underline{\mathrm{S} 281} ; \underline{\mathrm{S} 293} ; \underline{\mathrm{S} 295} ;$ \\
\hline & & & & & & & $\underline{\mathrm{S} 385} ; \mathrm{S} 410$ \\
\hline & A & 624 & & & 390 & & $\underline{\mathbf{S} 115}$ \\
\hline & C & 273 & & & 232 & & $\underline{Y 5} ; \mathrm{S} 17$ \\
\hline \multirow[t]{7}{*}{ Sm proteins } & $\mathrm{B} / \mathrm{B}^{\prime}$ & 352 & & & 516 & & $\underline{\mathrm{T} 30}$ \\
\hline & D1 & 108 & & & 177 & & \\
\hline & D2 & 393 & & & 209 & & $\mathrm{~T} 12 ; \underline{\mathrm{T} 40}$ \\
\hline & D3 & 346 & & & 339 & & $\underline{\mathrm{S} 2} ; \underline{\mathrm{S} 44}$ \\
\hline & E & 174 & & & 241 & & \\
\hline & $\mathrm{F}$ & 266 & & & 233 & S2 & S2 \\
\hline & G & 98 & & & 89 & & \\
\hline
\end{tabular}

The U1-70K protein is a heavily phosphorylated protein and its reversible phosphorylation is required for the first step of splicing $[137,138]$. We found 17 phosphorylation sites on U1-70K out of which 11 phosphorylation sites were not previously described. The C-terminal half of U1-70k, which contains the RS1 and RS2 regions and has been implicated in protein-protein interaction with SR proteins that mediate 5' splice site recognition [139], is identified to be phosphorylated on serine 245, 257, 259, 266, 268, 281, 293, 295 and 385. The U1-specific proteins (U1-A and $\mathrm{U} 1-\mathrm{C}$ ) and the common Sm proteins (B'/B, D2, D3 and F), which are important to splicing activity [140], are also found to be phosphorylated. A total of 38 phosphorylation sites corresponding to 9 SR proteins have been identified in this study. SR proteins facilitate the association of snRNPs with the pre-mRNA during spliceosome assembly, and phosphorylation is essential for SR protein activity $[116,117]$ and enhances their interactions with other spliceosomal proteins [118]. 


\subsection{Conclusion}

Our method opens up a new approach for large scale phosphoproteome study using calcium chloride and disodium hydrogen phosphate to precipitate low abundant phosphoproteins coupling with $\mathrm{TiO}_{2}$ to enrich phosphopeptides. The phosphoproteins precipitation by calcium chloride is more efficiently than magnesium chloride owing to the lower solubility product constants of calcium phosphate $\left(\mathrm{Ca}_{3}\left(\mathrm{PO}_{4}\right)_{2}, \mathrm{Ksp}=1 \times 10^{-32} ; \mathrm{Mg}_{3}\left(\mathrm{PO}_{4}\right)_{2}, \mathrm{Ksp}=1 \times 10^{-24}\right)$. Furthermore, we have demonstrated that several commercialyl available denatured reagents, SDS, urea and RapiGest TM SF, are compatible with our method. Coupling with in-house $\mathrm{TiO}_{2}$ microspin column increases the specificity toward phosphopeptides by reducing the amount of non-phosphorylated peptides. Even in complex biological samples such as U1 snRNPs, trace phosphopeptides derived from U1 snRNPs can be effectively identified by the CPP in combination with $\mathrm{TiO}_{2}$ enrichment. In summary, our CPP method for phosphoprotein isolation has several advantages - easy to use, sensitivity, selectivity and inexpensive, which can be strategically adapted for concerted extraction and pre-concentration of both non-phosphorylated and phosphorylated proteins prior to MS analysis in proteomic applications. 


\section{Chapter 4 - Pseudo-Neutral-Loss Scan for Selective Detection of Phosphopeptides and $\mathrm{N}$-Glycopeptides using Liquid Chromatography Coupled with a Hybrid Linear Ion-Trap / Orbitrap Mass Spectrometer}

\subsection{Summary}

In this study, we describe a novel method, termed "pseudo-neutral-loss scan", for selectively probing phosphopeptides and glycopeptides on a hybrid LTQ-Orbitrap mass spectrometer (MS). The instrument has been programmed such that phosphopeptides or glycopeptides eluted from reverse phase liquid chromatography (LC) can automatically be discovered and identified in a way similar to that of the use of neutral-loss scanning. An in-source collision-induced dissociation (CID) energy is applied on all species ionized in electrospray ion source, resulting in a neutral loss(es) of phosphoric acid or monosaccharide residues. Subsequently, the characteristic mass pair that differ in the mass of a neutral loss of phosphoric acid or monosaccharide residues are automatically selected for CID MS/MS to obtain modified peptide sequence information. However, the CID MS/MS for glycopeptide predominantly generates fragment ions from cleavages of glycosidic bonds without breaking the peptide bonds. Therefore, a targeted MS3 acquisition for peptide+HexNAc ion is directly following the MS/MS scan to collate identification and characterization of the glycopeptides in one experimental scan cycle. Performing the experiment in the LTQ-Orbitrap enables subsequent high resolution / high mass accuracy full scan in-source CID mass spectra. As the accurate mass pair of neutral loss is pre-determined (error within $5 \mathrm{ppm}$ ), the enormous number of MS/MS scans on non-paired species can be greatly reduced. Our method is compatible with nano-LC for separation of complex peptide mixtures without any further enrichment, resulting in a highly selective and sensitive approach to identify phosphopeptides and glycopeptides from a purified protein digestion, and could be potentially be of use in more protein mixture. The consequent spectra provide peptide sequence identification and modified site assignment as well as information of the glycan structure.

\subsection{Introduction}

Post-translational modifications (PTMs) of proteins modulate the activity of the proteins, protein-protein and protein-ligand interaction within cells. Analysis of PTMs presents immense analytical challenges, but their identification has led to essential knowledge and understanding of biological function. Because of its sensitivity and selectively, mass spectrometry (MS) is currently the most versatile technology to determine PTMs directly. However, the identification of PTMs still remains a substantial challenge owing to the low abundance of modified peptides and thus the relatively large amounts of non-modified peptides in samples; the excess of non-modified peptides results in suppression of the ion signal of the modified peptides in MS [58]. Protein phosphorylation and glycosylation is of particular interest since it is the most 
common protein modification shown in Figure 4.1.

(A)

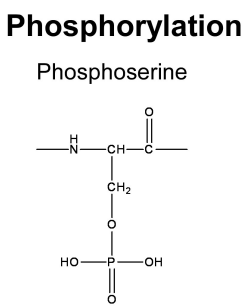

(B) $\mathbf{N}$-Glycosylation

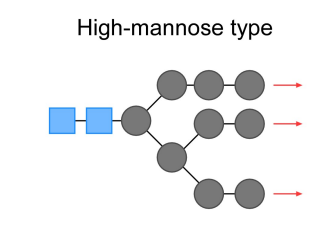

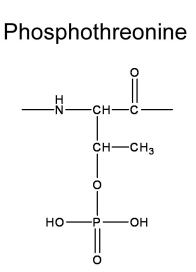

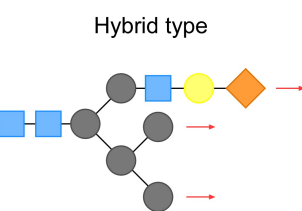

\section{Glycosylation}

O-Glycosylation

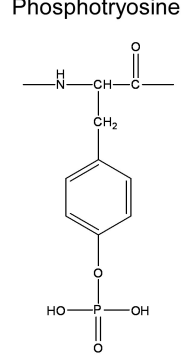

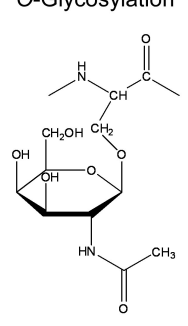

N-Glycosylation

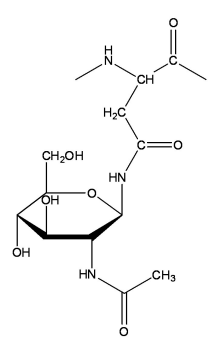

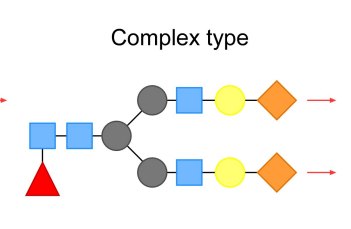

N-acetylglucosamine (GalNac)

Mannose (Man) Galactose (Gal)

Sialic acid (SA)

Fucose (Fuc)

Figure 4.1. The common covalent post-translational modifications - phosphorylation and glycosylation. (A) Phosphorylation, the phosphate group covalently links to serine, threonine or tyrosine residue. O-glycosylation, the $\mathrm{N}$-acetylgalactosamine covalent links to serine or threonine residue. $\mathrm{N}$-glycosylation, the $\mathrm{N}$-acetylgalactosamine covalent links to asparagine residue. (B) There are three major classes of $\mathrm{N}$-glycosylation protein in mammalian cell, high-mannose type, complex type and hybrid type.

Reversible phosphorylation of proteins is an important regulatory mechanism in a wide range of cellular processes (see chapter 2 and 3). Glycosylation plays a crucial role not only in protein structure but also in cell-cell recognition, and perhaps even signaling mechanisms [141]. Hence, the analysis of protein glycosylation is essential if its biological roles are to be understood and evaluated. To completely describe protein glycosylation, three sections of information are required: (1) identification of the glycosylated proteins and peptides, (2) the position of the glycosylation, and (3) the structure of the glycan. O-glycosylation occurs at the side chain of serine or threonine residues during the later stage of protein processing, probably in the Golgi apparatus [142, 143]. N-glycosylation generally occurs at the side chain of asparagine where Asn is a part of the triplet Asn-X-Ser/Thr, where $\mathrm{X}$ can be any amino acid residue except proline [144, 145]. The process of oligosaccharide attached to asparagine of protein migrates through the endoplasmic reticulum (ER) and the Golgi apparatus, leading to the formation of high-mannose, complex, or hybrid type glycoprotein in mammalian cells, The high-mannose type contains two $\mathrm{N}$-acetylglucosamine and many of mannose residues, The complex type contains two $\mathrm{N}$-acetylglucosamines, three mannoses, and additional $\mathrm{N}$-acetylglucosamine, galactose, sialic acid, and fucose residues. The hybrid type has both high-mannose and complex type characteristics [146, 147]. The glycosylation modification is 
highly heterogeneous, making them challenging for characterization in MS analysis. The most common approach for identification of phosphorylation and glycosylation is the separation technology such as affinity-based enrichment of phosphopeptides [23] or glycopeptides [24] before MS analysis. An alternative isolation strategy, demonstrated as being highly successful, is designated to the precursor ion and the neutral loss scanning techniques utilising electrospray ionization (ESI) in a tandem mass spectrometer (MS/MS) shown in Figure 4.2.

(A)
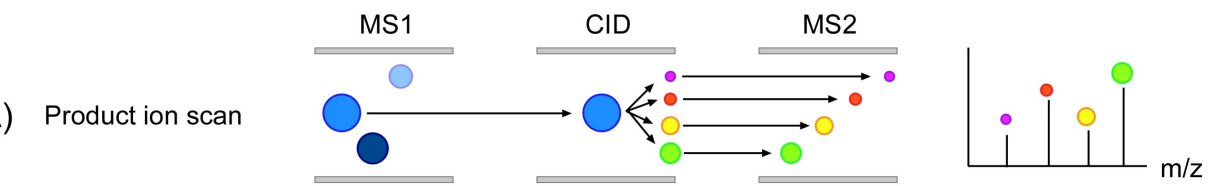

(B) Precursor ion scan

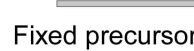

Scan product
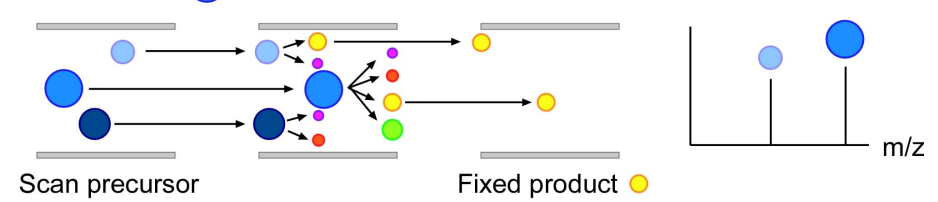

Fixed product

(C) Neutral loss scan
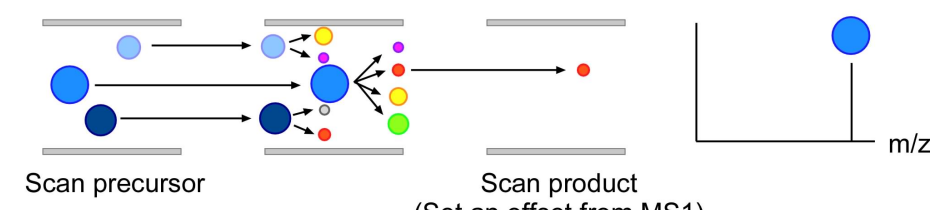

Scan product (Set an offset from MS1)

(D) Multiple reaction monitoring
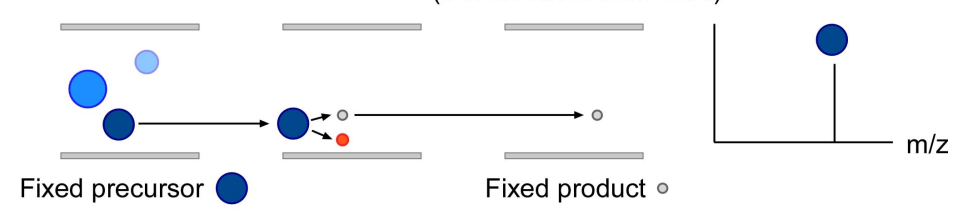

Fixed product $。$

Figure 4.2. The scheme of various types of tandem mass spectrometry (MS/MS) experiments. (A) Product ion scan is the most common MS/MS experiment in proteomic research. The first MS selects one specific precursor ion. The selected ion undergoes collision-induced dissociation (CID) in the collision cell, and the resulting fragment ions are analyzed by the second MS. The spectra can be used for identification of peptide sequences. (B) Precursor ion scan is used to analyze a subset of peptides containing a specific function group such as a phosphate or a saccharide residue. The first MS is making a regular full scan. All ions that pass through the first MS are fragmented in the collision cell. The MS2 is fixed, allowing to transmit only one specific fragment ion. (C) Neutral loss scan, all precursor ions that undergo the neutral loss of a specific functional group are monitored. The neutral fragment is lost in the collision cell. The first MS scans all masses and the second MS also scans, but at a set offset from the first MS. This offset corresponds to a neutral loss that is commonly observed for the class of compounds, such as a neutral loss of phosphoric acid via beta-elimination in CID for identification of protein phosphorylation at serine and threonine residue. (D) Multiple reaction monitoring (MRM) is used for quantification of targeted analytes with the known fragmentation properties. The first MS allows multiple masses through and the second MS monitors for specific user defined fragment ions. Use of MRM increases selectivity, improves $\mathrm{S} / \mathrm{N}$ ratio. 
Modified peptides can be recognised from a common diagnostic fragment ion ( $\mathrm{m} / \mathrm{z}$ at 216 for phosphotyrosine immonium ion) [148, 149] or neutral loss mass (loss of $98 \mathrm{Da}$ in the case of phosphoserine or phosphothreonine) [150,151], as well as characteristic neutral losses of carbohydrates on glycosylated peptides (loss of $162 \mathrm{Da}$ or $203 \mathrm{Da}$ in the case of Hex or HexNAc, respectively) [152]. In the case of a neutral-loss experiment, the $\mathrm{m} / \mathrm{z}$ ratios of ion pairs are selectively determined that exhibits the mass difference of the neutral component (e.g. $\mathrm{H}_{3} \mathrm{PO}_{4}$ or Deoxyhexose (DeoxyHex), Hexose (Hex), $\mathrm{N}$-acetylhexosamine (HexNAc), $\mathrm{N}$-acetylneuraminic acid (NeuAc) or $\mathrm{N}$-glycolylneuraminic acid (NeuGc)). The most common MS set-up for recording precursor-ion and neutral-loss scans is a triple quadrupole MS, in which the single quadrupoles can selectively and rapidly scan for $\mathrm{m} / \mathrm{z}$ ion pairs. However, "real" neutral-loss scan experiments coupled with on-line LC on quadrupole time-of-flight (Q-TOF), ion-trap or Fourier-transform ion cyclotron resonance (FT-ICR) MS instruments are not possible, because the experiments require two continuous scanning mass analyzers with an offset of neutral loss. In this study, we developed a novel scan mode in the LTQ-Orbitrap. This we call a pseudo-neutral-loss scan, as it mimics the neutral-loss scan in triple-quadrupole MS. It shows potential value in the identification of phosphorylated and $\mathrm{N}$-glycosylated sites on proteins.

\subsection{Experiment Sections}

\subsubsection{Materials}

Ammonium bicarbonate, beta-casien, Chloroacetamide (CAA) and ribonuclease B (RNase B) were obtained from Sigma-Aldrich (St. Louis, MO). Sequencing grade, modified trypsin was obtained from Promega (Madison, WI). RapiGest ${ }^{\mathrm{TM}} \mathrm{SF}$ was obtained from Waters Corporation (Manchester, UK). Dithiothreitol (DTT), formic acid and acetonitrile (ACN) were obtained from Merck (Darmstadt, Germany).

\subsubsection{In-Solution Digestion}

The standard protein was dissolved in $10 \mu \mathrm{l} 1 \%$ RapiGest $^{\mathrm{TM}} \mathrm{SF}$. The protein solution was reduced with $10 \mu \mathrm{l}$ of $10 \mathrm{mM} \mathrm{DTT}$ at $37{ }^{\circ} \mathrm{C}$ for 1 hour, alkylated with $10 \mu \mathrm{l}$ of $20 \mathrm{mM}$ CAA at 37 ${ }^{\circ} \mathrm{C}$ for 1 hour, diluted to $0.1 \%$ RapiGest ${ }^{\mathrm{TM}} \mathrm{SF}$ with $70 \mu \mathrm{l}$ of $25 \mathrm{mM}$ ammonium bicarbonate, and subsequently digested with either trypsin at $37{ }^{\circ} \mathrm{C}$, overnight (the enzyme to substrate ratio is $1: 20$ ). The resulting peptides were acidified with $50 \mu$ of $5 \%$ TFA at $37{ }^{\circ} \mathrm{C}$ for 2 hours and centrifuged at $17000 \mathrm{~g}$ for $15 \mathrm{~min}$. Subsequently, the supernatant was transferred to another eppendorf and dried down by SpeedVac for further MS analysis.

\subsubsection{Mass Spectrometry}

MS analysis was performed with a LTQ-Orbitrap mass spectrometer (Thermo Fisher Scientific), 
operated under Xcalibur Software 2.0.7, equipped with a nanoelectrospray ion source and coupled to an Agilent 1100 HPLC system (Agilent Technologies), fitted with a C18 column made in-house. Tryptic peptides were first loaded at a flow rate of $10 \mu \mathrm{L} / \mathrm{min}$ onto a C18 trap column (1.5 cm, $360 \mu \mathrm{m}$ o.d., $150 \mu \mathrm{m}$ i.d., Reprosil-Pur $120 \AA$, $5 \mu \mathrm{m}, \mathrm{C} 18-\mathrm{AQ}$, Dr. Maisch $\mathrm{GmbH}$, Germany). Retained peptides were eluted and separated on an analytical $\mathrm{C} 18$

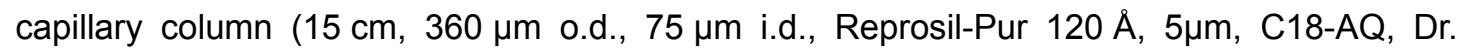
Maisch $\mathrm{GmbH}$, Germany) at a flow rate of $300 \mathrm{~nL} / \mathrm{min}$, with a gradient from 7.5 to $37.5 \% \mathrm{ACN}$ in $0.1 \%$ formic acid for $60 \mathrm{~min}$. Typical MS conditions were: spray voltage, $1.7 \mathrm{kV}$; heated capillary temperature, $150^{\circ} \mathrm{C}$; normalised collision-induced dissociation (CID) collision energy $37.5 \%$ for MS/MS in LTQ. An activation $q=0.25$ and activation time of $30 \mathrm{~ms}$ were used. The Xcalibur Software 2.0.7 implements all the functions we performed in this study, called "source fragmentation", "use $\mathrm{m} / \mathrm{z}$ values as masses", "mass tags" and "multistage activation". The function of source fragmentation allows to turn on and off ion source collision-induced dissociation (CID) of a specified scan event. The function of use $\mathrm{m} / \mathrm{z}$ values as masses determines the charge state of the ionized species from the full MS spectrum and converts the mass-to-charge ratios into masses. The function of mass tags allows a pair of mass tagged ions is to trigger a MS/MS scan. The function of multistage activation is the process of collapsing one or more neutral loss MS3 experiments down to a single MS/MS experiment. For phosphopeptide analysis, the mass spectrometer was automatically switched between MS and MS/MS acquisition when the neutral loss of phosphoric acid was detected. Survey full scan MS spectra (from m/z 350-1600) were acquired in the Orbitrap with resolution $R=30,000$ at $\mathrm{m} / \mathrm{z} 400$ (after accumulation to a 'target value' of 1,000,000 in the Orbitrap), with an in-source CID energy of $70 \mathrm{~V}$, and the option of $\mathrm{m} / \mathrm{z}$ value as masses was enabled. The option of mass tags was enabled and the value was set at 97.9769 . The mass-pair ions were isolated sequentially and fragmented in the linear ion trap by using multi-stage activation CID MS/MS at a target value of 100,000 [132]. For glycopeptide analysis, the mass spectrometer was automatically switched between MS and MS/MS acquisition when the neutral loss of monosaccharide residues was detected. Survey full-scan MS spectra ( $\mathrm{m} / \mathrm{z}$ from 150 to 1600$)$ were acquired in the Orbitrap with resolution $R=30,000$ at $\mathrm{m} / \mathrm{z} 400$ (after accumulation to a 'target value' of $1,000,000$ in the Orbitrap), and an in-source CID energy of $30 \mathrm{~V}$. The mass range for selecting MS-data-dependent masses was set 1500-1,000,000, and the option of $\mathrm{m} / \mathrm{z}$ value as masses was enabled. The option of mass tags was enabled and the value was set at 146.0579, 162.0528, 203.0794, 291.0954, 307.09033 for DeoxyHex, Hex, HexNAc, NeuAc and NeuGc, respectively. The mass-pair ions were isolated sequentially and fragmented in the linear ion trap using CID MS/MS at a target value of 100,000 . The theoretical $\mathrm{m} / \mathrm{z}$ of peptide+HexNAc was entered in the product mass list for triggering multi-stage activation CID MS3, limiting the selection of ions for data-dependent acquisition to the top 20 ion intensities. 


\subsection{Result and Discussion}

\subsubsection{The Principle of Pseudo-Neutral-Loss}

Our method is based on the presence of an ion pair of known characteristic mass in the mass spectrum that is generated during in-source CID fragmentation. If the signals of the mass pair exhibited in the MS spectrum correspond to the representative neutral loss (phosphoric acid or monosaccharide residues, see below), these ions can be selected for MS/MS shown in Figure 4.3.

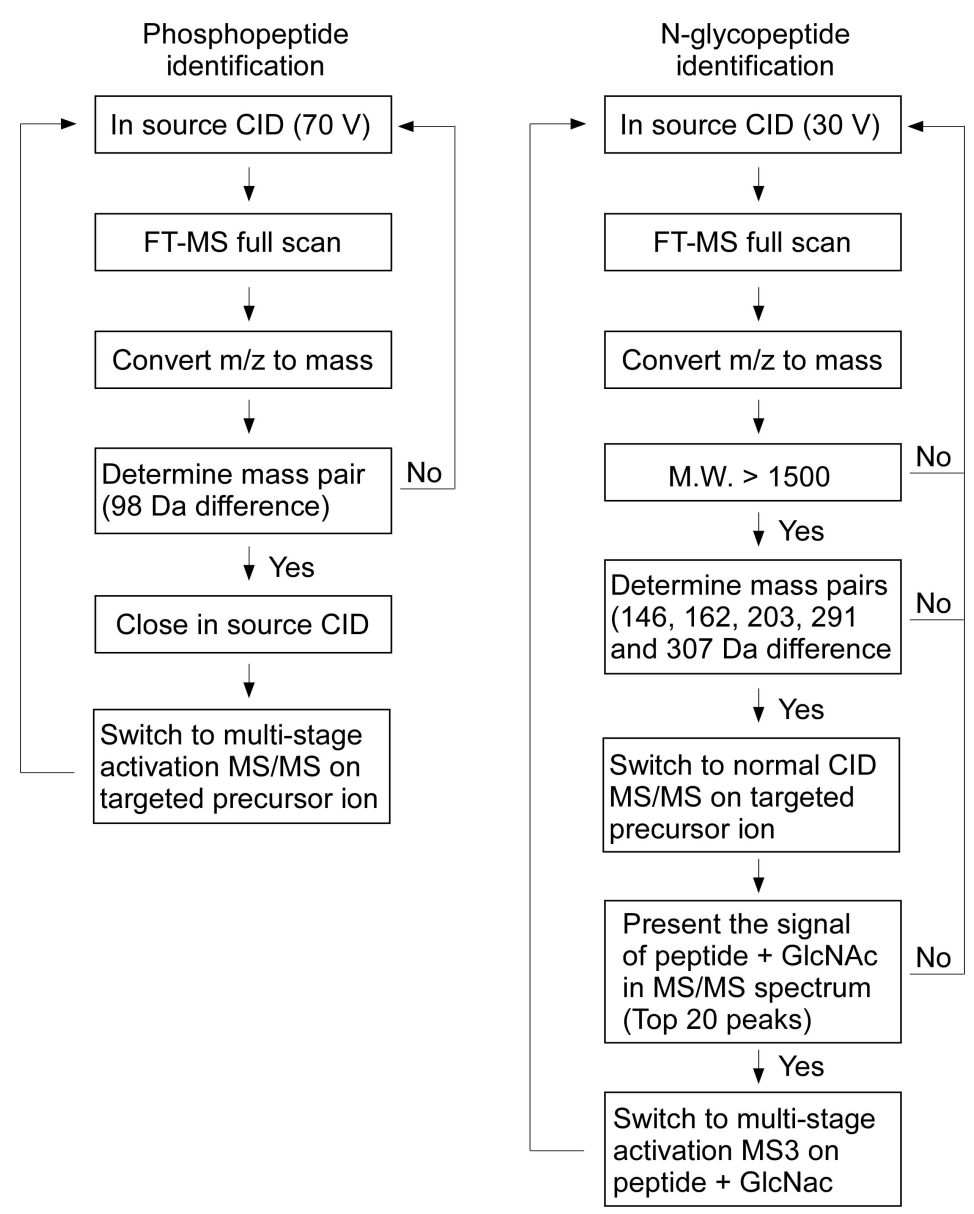

Figure 4.3. Workflow of pseudo-neutral-loss scan in LTQ-Orbitrap for the detection of phosphorylated peptides and $\mathrm{N}$-glycosylated peptides.

For phosphopeptide identification on peptides that elute from the LC into the orifice of the Orbitrap MS, an additional potential difference of $70 \mathrm{~V}$ in the ion transfer region is applied for in-source CID fragmentation; this removes phosphoric acid on phosphoserine and phosphothreonine residues by charge directed of neutral loss [153, 154]. The LTQ-Orbitrap instrument determines the charge state of the ionized species from the full MS spectrum and converts the mass-to-charge ratios into masses. If a mass pair corresponding to the loss of 
neutral phosphoric acid (98 Da) is present in the full scan mass spectrum and its intensity is above the user-defined data-dependent threshold, the collision energy in the ion source is turned off, and multi-stage activation MS/MS is triggered subsequently for the corresponding selected mass. Importantly, the high mass accuracy of the LTQ-Orbitrap mass spectrometer allows the neutral loss of phosphoric acid to be specified within an error of $5 \mathrm{ppm}$. Operating under this strict criterion dramatically reduces the number of false positives as encountered earlier in triple- quadrupole instruments.

For the identification of $\mathrm{N}$-glycopeptides, an additional potential difference in the ion transfer region is set to $30 \mathrm{~V}$, which is sufficient to produce marker ions at $\mathrm{m} / \mathrm{z}=204\left(\mathrm{HexNAc}^{+}\right), 292$ $\left(\mathrm{NeuAc}^{+}\right)$, and $366\left(\mathrm{Hex}-\mathrm{HexNAc}{ }^{+}\right)$[155]. Monitored mass-to-charge ratios are then converted into masses. The setting of precursor mass selection for MS/MS sequencing is applied only to deconvoluted masses that exceed $1500 \mathrm{Da}$, which is the minimum molecular weight of mammalian tryptic $\mathrm{N}$-glycopeptides. Under reverse-phase (RP) LC conditions, chromatograms of heterogeneous glycopeptides harbouring the same peptide sequence typically show multiple co-eluting glycosylated forms in different mass and/or charge states [156]. This is because the oligosaccharide moieties are hydrophilic and the peptide - depending on its sequence - is hydrophobic, and hydrophobicity of the peptides can be regarded as main reason for separation under RP conditions. Therefore, the appearance of mass pairs in the MS full-scan spectrum corresponds either to the co-elution of glycopeptides that contain different carbohydrate moieties or to the neutral loss of monosaccharide residues by in-source CID (DeoxyHex, 146 Da; Hex, 162 Da; HexNAc, 203 Da; NeuAc, 291 Da; NeuGc, 307 Da). Accordingly, all species that reveal the given mass difference in the deconvoluted full-scan mass spectrum are selected for CID MS/MS. However, the MS-based analysis of glycopeptides using CID fragmentation is difficult, as it typically reveals predominantly information on the composition and sequence of the glycan moiety, without revealing any information about the peptide sequence. Therefore, an additional MS3 scan is required to obtain information about the peptide. To select for fragments within the MS/MS that are suitable for MS3, we made use of the fact that $N$-glycosylated peptides with the known consensus motif Asn-X-Ser/Thr, (where $\mathrm{X}$ can be any amino acid except Pro) always carry HexNAc as the first sugar at Asn-residue. This allows one to calculate the theoretical mass of a [peptide-HexNAc] in order to then trigger the multi-stage activation MS3 and finally to obtain a peptide fragmentation pattern.

\subsubsection{Identification of Phosphopeptide by Pseudo-Neutral-Loss Scan}

To demonstrate our working scheme for the various neutral losses, we first carried out a preliminary pseudo-neutral-loss scan experiment for the identification of phosphorylation sites by using $10 \mathrm{fmol}$ tryptic peptides on column derived from tryptic digestion of beta-casein. 
Beta-casein is frequently used as a model phosphoprotein in the development of MS methods for analysis of phosphorylation sites [71, 75, 133]. At first, we optimised the in-source CID energy in order to generate fragment ions showing neutral loss of phosphoric acid $\left(\mathrm{H}_{3} \mathrm{PO}_{4}\right)$. We examined the in-source CID energy from $0 \mathrm{~V}$ to $80 \mathrm{~V}$ as shown in Figure 4.4.

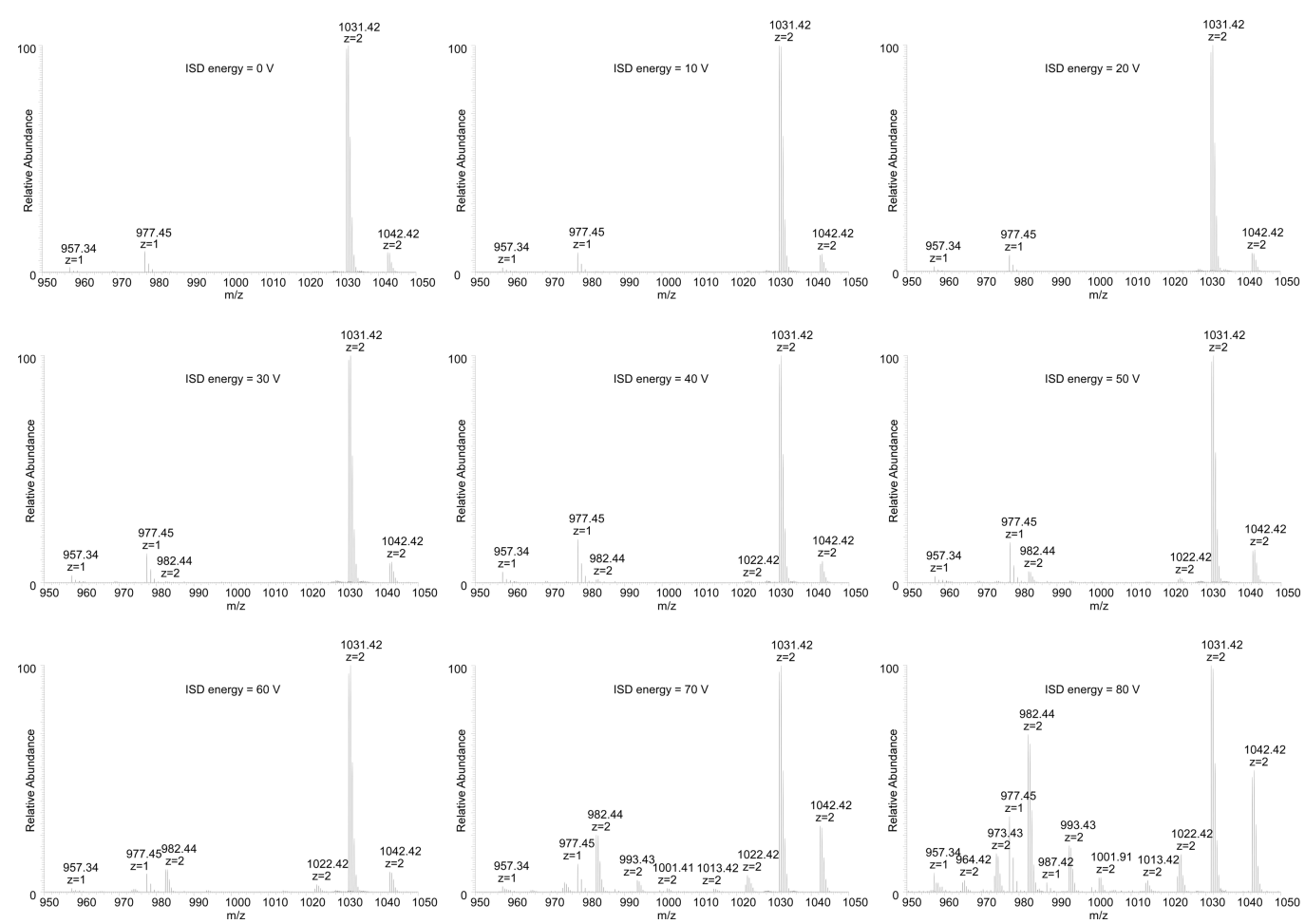

Figure 4.4. Optimisation of in-source CID energy for phosphopeptide detection with pseudo-neutral-loss scan in LTQ-Orbitrap. Mass spectra were acquired with in-source $\mathrm{CID}$ energies from $0 \mathrm{~V}$ to $80 \mathrm{~V}$. The signal at $\mathrm{m} / \mathrm{z} 982.44(2+)$ corresponds to the neutral loss of phosphoric acid on the phosphopeptide ${ }^{48} \mathrm{FQpSEEQQQTEDELQDK}{ }^{63}$ of beta-casein at $\mathrm{m} / \mathrm{z} 1031.42(2+)$.

Below $20 \mathrm{~V}$, the signal at $\mathrm{m} / \mathrm{z} 982.44\left[{ }^{48} \mathrm{FQpSEEQQQTEDELQDK}{ }^{63}-\mathrm{H}_{3} \mathrm{PO}_{4}\right]$, corresponding to neutral loss of phosphoric acid on the phosphopeptide of beta-casein at $\mathrm{m} / \mathrm{z} 1031.42$ $\left[{ }^{48} \mathrm{FQpSEEQQQTEDELQDK}{ }^{63}\right]$ was not present in the spectra. When the energy was raised to $30 \mathrm{~V}$, a weak signal of the corresponding neutral loss ion started to be detectable. Note that the relative intensity ratio of neutral-loss ion to precursor ion was in direct proportion to the in-source CID energy. The maximum signal of neutral-loss ion was observed at $80 \mathrm{~V}$; however, many peptide backbone cleavages were already generated under these conditions, resulting in the significant decrease in the signal intensity of phosphopeptide as compared with $70 \mathrm{~V}$, as shown in Figure 4.5. The signal of the phosphopeptide dropped approximately $15 \%$ and $70 \%$ at $70 \mathrm{~V}$ and $80 \mathrm{~V}$, respectively. We therefore set the in-source CID energy to $70 \mathrm{~V}$ for further analysis. 

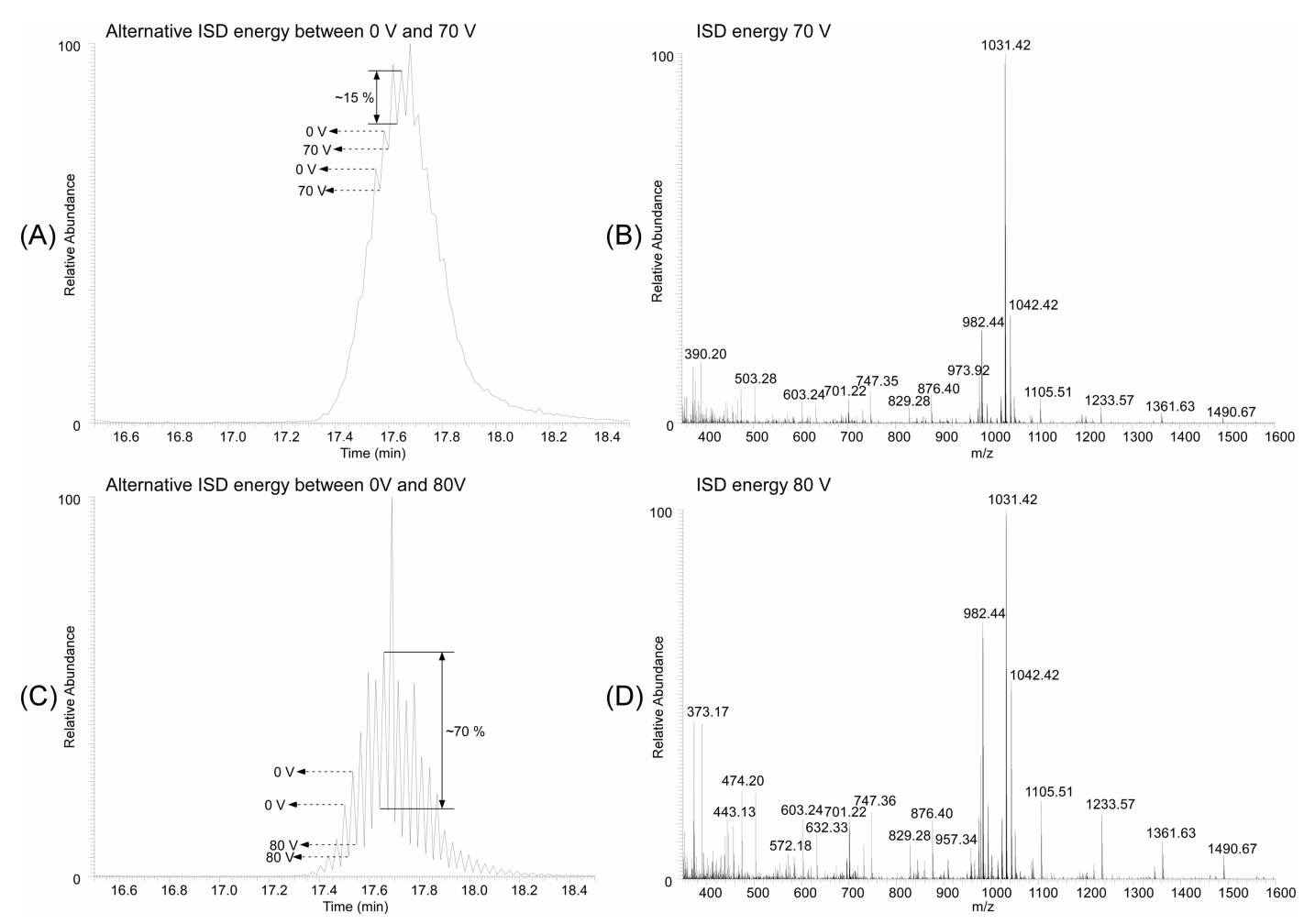

Figure 4.5. (A) Extracted ion chromatography of the phosphopeptide ${ }^{48} \mathrm{FQPSEEQQQTEDELQDK}{ }^{63}$ of beta-casein at $\mathrm{m} / \mathrm{z} 1031.42$ acquired with an alternative in-source CID energy between $0 \mathrm{~V}$ and $70 \mathrm{~V}$. (B) Mass spectrum under in-source CID energy $70 \mathrm{~V}$. (C) and (D) An alternative in-source CID energy was set to $0 \mathrm{~V}$ and $80 \mathrm{~V}$ instead of $0 \mathrm{~V}$ and $70 \mathrm{~V}$. The signal of phosphopeptide dropped by approximately $15 \%$ and $70 \%$ to below $70 \mathrm{~V}$ and $80 \mathrm{~V}$, respectively, owing to peptide backbone cleavage.

As illustrated in the workflow (Figure 4.3), the appearance of the neutral loss of phosphoric acid in the case of precursors containing phosphoserine and phosphothreonine under high in-source CID energy caused the instrument to switch automatically into the product-ion mode (MS/MS) to acquire peptide-sequence information. This step involved measuring the masses of the precursor ions, determining their charge states, identifying the neutral loss of phosphoric acid and generating a list of precursor masses for MS/MS sequencing. Figure 4.6 shows the workflow for the identification of the phosphopeptide ${ }^{48} \mathrm{FQpSEEQQQTEDELQDK}{ }^{63}$ of beta-casein using nanoLC coupled with a pseudo-neutral-loss scan in the LTQ-Orbitrap. When an in-source CID energy was employed at $70 \mathrm{~V}$, a neutral loss of phosphoric acid (48.99) at $\mathrm{m} / \mathrm{z} 982.43$ derived from a doubly charged phosphopeptide precursor at $\mathrm{m} / \mathrm{z} 1031.42$ was observed (Figure 4.6A). The mass-to-charge ratios were converted into masses (Figure 4.6B). The presence of a mass pair equivalent to a neutral loss of phosphoric acid (97.98 Da) was selected for CID MS/MS to obtain peptide sequence information. To generate fragment ions of the phosphopeptides that were structurally more informative, "multi-stage activation" [132] corresponding to a neutral loss of phosphoric acid from doubly and triply charged precursor 
ions was enabled in all MS/MS events and identified the phosphorylation as being on serine 50 (Figure 4.6C). The pseudo-neutral-loss scan of phosphoric acid greatly reduced the large number of false positive candidate precursor ions based on the highly accurate mass pair (Figure 4.6D), as compared with regular data-dependent acquisition (DDA) (Figure 4.6E).

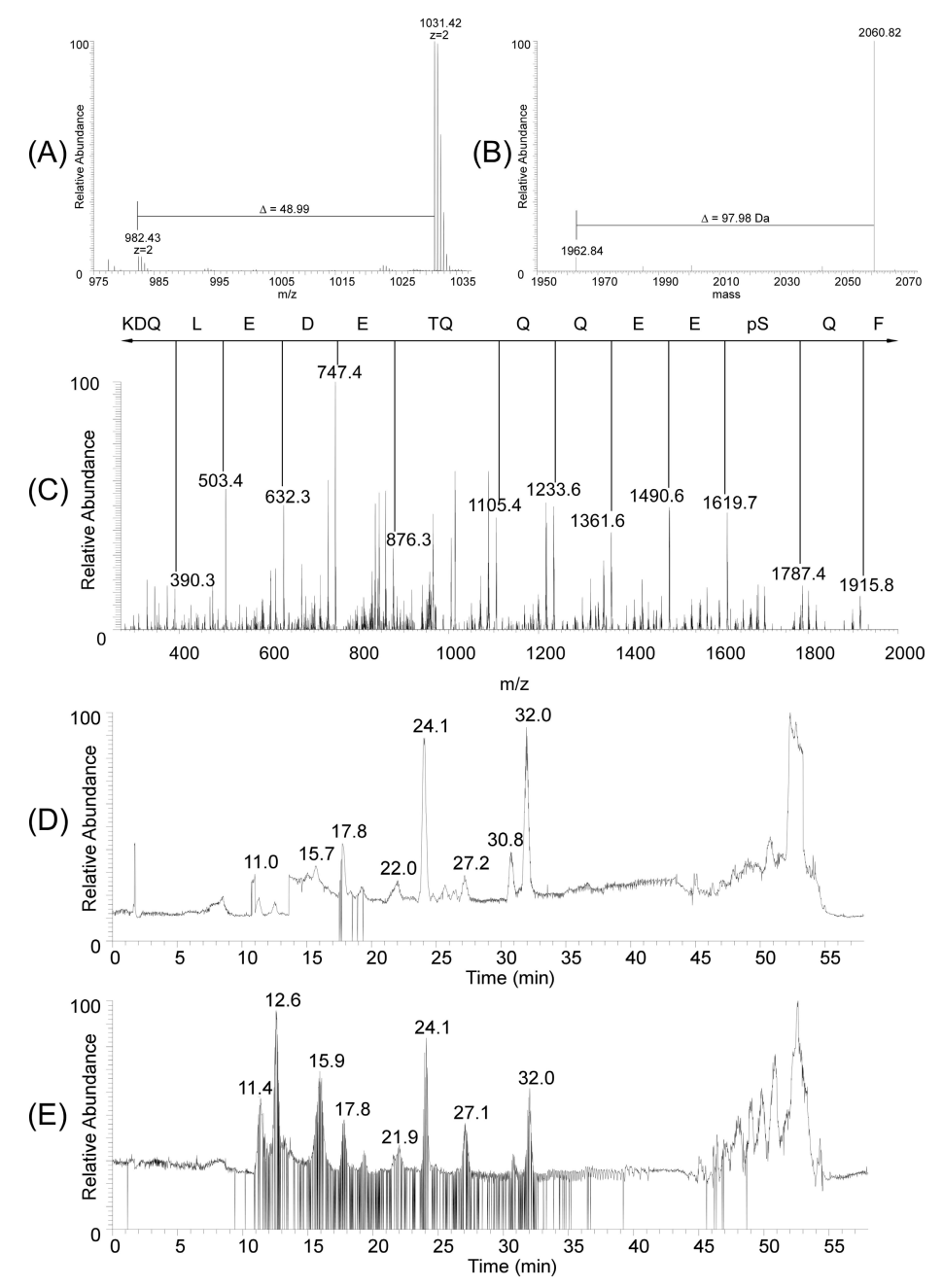

Figure 4.6. Identification of tryptic phosphopeptide ${ }^{48} F Q p S E E Q Q Q T E D E L Q D K{ }^{63}$ from beta-casein using a pseudo-neutral-loss scan. (A) A full mass spectrum obtained from the LTQ-Orbitrap mass spectrometer at an in-source CID energy of $70 \mathrm{~V}$, the difference in $\mathrm{m} / \mathrm{z}$ value of 48.99 corresponding to the neutral loss of doubly charged phosphoric acid. (B) A deconvoluted and deisotoped mass spectrum based on direct assignment of charge to the measured signal from Figure 4.6A. (C) Pseudo-neutral-loss scan of phosphoric acid (97.98 Da) triggered multi-stage activation MS/MS acquisition for phosphopeptide ${ }^{48} \mathrm{FQpSEEQQQTEDELQDK}{ }^{63}$ at $\mathrm{m} / \mathrm{z}$ 1031.42. (D) The total ion chromatogram obtained from pseudo neutral loss scan, in which MS/MS was triggered only the mass pair with pre-defined neutral loss of phosphoric acid identified in the MS scan. The phosphopeptide ${ }^{48} \mathrm{FQpSEEQQQTEDELQDK}{ }^{63}$ was eluted and was identified at retention time $17.51 \mathrm{~min}$. (E) The total ion chromatogram generated from regular data-dependent acquisition. 
We also examined the power of our method by analyzing tryptic peptides derived from a mixture of $10 \mathrm{fmol}$ beta-casein and $50 \mathrm{fmol} \mathrm{BSA} \mathrm{(Figure} 4.7$ and Appendix 4). Despite of the fact that the phosphopeptide ${ }^{48} \mathrm{FQpSEEQQQTEDELQDK}{ }^{63}$ at $\mathrm{m} / \mathrm{z} 1031.42$ was present in the regular DDA (Supplementary Figure 4.7C), however, the signal was not intensive to be triggered MS/MS. Compared with regular DDA, pseudo-neutral-loss acquisition could identify the phosphopeptide ${ }^{48} \mathrm{FQPSEEQQQTEDELQDK}{ }^{63}$ by using MASCOT database search, and the result indicated large amount of non-phosphorylated peptides derived from BSA and beta-casein were removed (Appendix 4).
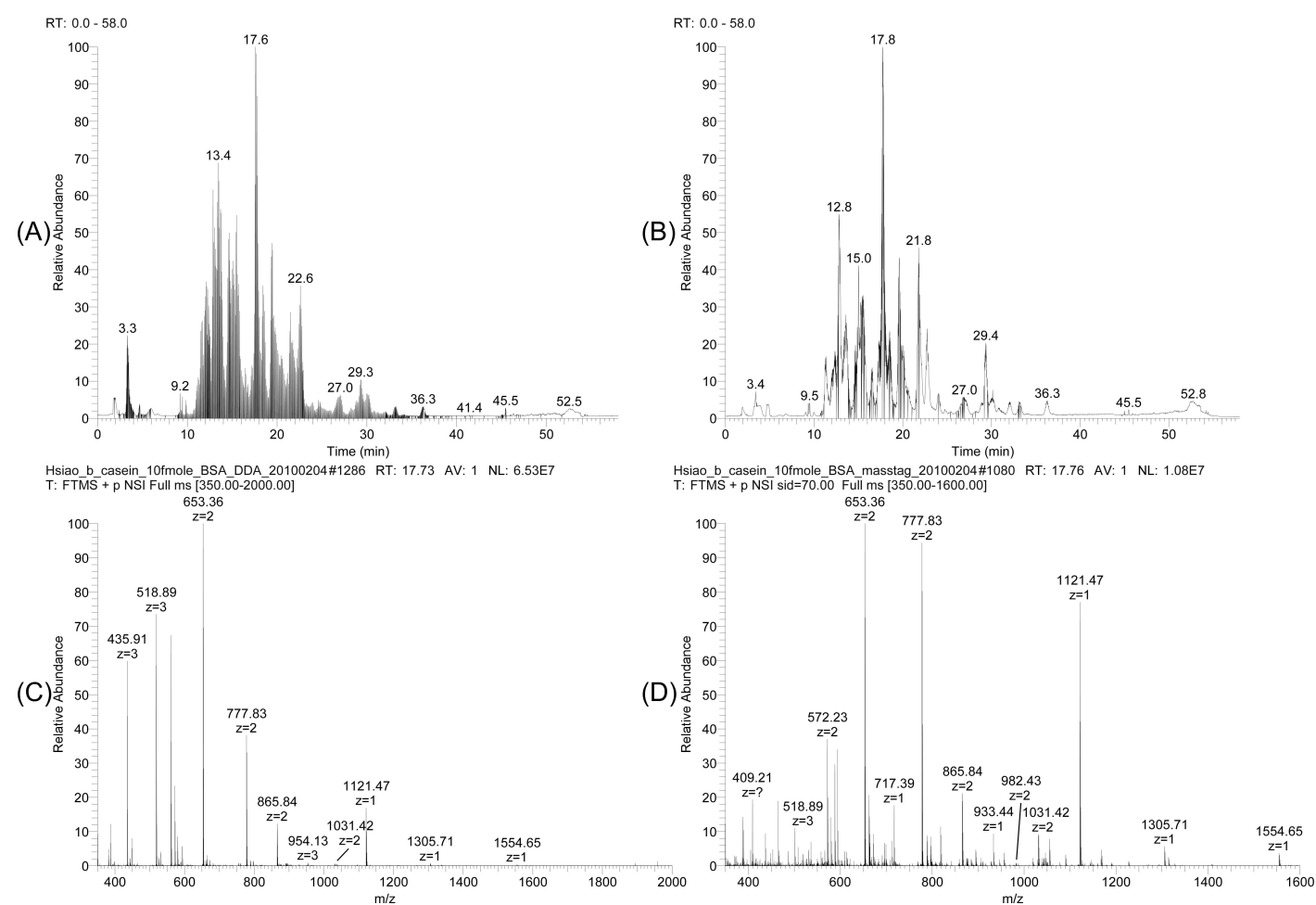

Figure 4.7. Identification of tryptic phosphopeptide ${ }^{48} \mathrm{FQpSEEQQQTEDELQDK}{ }^{63}$ at $\mathrm{m} / \mathrm{z} 1031.42$ derived from $10 \mathrm{fmol}$ beta-casein containing $50 \mathrm{fmol}$ BSA tryptic peptides. (A) Regular data-dependent acquisition. (B) Pseudo-neutral-loss acquisition. (C) A full scan mass spectrum obtained from $(A)$ at retention time $17.73 \mathrm{~min}$. (D) A full scan mass spectrum obtained from $(B)$ at retention time $17.76 \mathrm{~min}$. The mascot searching result showed the phosphopeptide ${ }^{48} \mathrm{FQpSEEQQQTEDELQDK}{ }^{63}$ only could be identified by using pseudo-neutral-loss acquisition and large amount of non-phosphorylated peptides derived from BSA and beta-casein were removed (Appendix 4).

\subsubsection{Identification of $\mathbf{N}$-Glycopeptide by Pseudo-Neutral-Loss Scan}

The performance of pseudo-neutral-loss scans for the detection of glycopeptides is particularly attractive because mass pairs of monosaccharide residues are readily obtained during the 
in-source CID fragmentation. However, since in-source CID causes the neutral loss of different monosaccharides attached to a peptide, the full MS spectrum will necessarily reveal several mass pairs. On the other hand, front-end separation of glycopeptides under reversed-phase conditions cannot separate peptide populations that carry a heterogeneous carbohydrate moiety; consequently, the mass pairs observed after in-source CID are due either to the stepwise neutral loss of a single modified peptide or to the presence of a naturally occurring heterogeneous peptide population eluting into the instrument. Despite this ambiguity under our liquid-chromatography conditions, it is nonetheless worthwhile to investigate the potential of pseudo-neutral-loss scanning of the monosaccharide residues following CID MS/MS in the ion trap combined with further multi-stage activation MS3 experiments as it makes our in-source CID essentially universal for glycopeptide identification and glycan structure profiling.

Figure 4.8 shows the results of a glycopeptide analysis from a 25 fmol tryptic digestion of ribonuclease $B$ (RNase $B$ ) loaded onto a nanoLC ESI-Orbitrap with an in-source CID energy of $30 \mathrm{~V}$. In Figure 4.8A, the mass spectrum shows a peptide that contains a high-mannose type $\mathrm{N}$-linked oligosaccharides (HexNAc2Man4 to HexNAc2Man9), and thus further demonstrates that different glycan structures on the same peptide backbone either co-eluted from the RP column or were generated by in-source CID (see above). Consequently, the presence of a mass pair due to the loss of mannose (162 and/or $203 \mathrm{Da}$ ) switched the LTQ into the MS/MS mode to record its product ion spectrum (Figure 4.8B). When the ion with $\mathrm{m} / \mathrm{z} 927.4$ was taken as a precursor for CID MS/MS, the monosaccharide ladder served to outline oligosaccharide structures (HexNAc2Man6) with an interval of 162 or 203 for singly charged ions or 81 and 101.5 for doubly charged ions. The ions for the peptide moiety $(\mathrm{m} / \mathrm{z} 475.4)$ and the [peptide-HexNAc] conjugate $(\mathrm{m} / \mathrm{z}$ 658.4) were also present in the spectrum. Note that the collision-induced dissociation in LTQ often led to preferential cleavage of the glycosidic bonds rather than of the polypeptide bonds and, as a result, it usually provides information primarily about the glycan structure and not about the peptide sequence. Subsequently, a targeted MS3 of the [peptide-HexNAc] ion at $\mathrm{m} / \mathrm{z} 678.4$ was carried out (Figure $4.8 \mathrm{C}$ ). The b2 and b3 ions at $\mathrm{m} / \mathrm{z} 431.4$ and 532.3 both retained the $N$-linked GlcNAc, and in combination with other product ions revealed the amino-acid sequence NLTK, where $\mathrm{N}$ was the glycosylation site of RNase $\mathrm{B}$. The neutral loss of HexNac at m/z 475.4 was the dominant signal of the [peptide-HexNAc] MS/MS spectrum (Figure 4.8C). To improve the peptide sequence coverage, a multi-stage activation MS3 corresponding to a neutral loss of HexNAc was employed to fragment the [peptide-HexNAc] (Figure 4.8D). Instead of regular CID MS3, we observed clearly an increase in the abundance of nearly every b/y-type ion, along with y2, y3, b2 and b3 ions at $\mathrm{m} / \mathrm{z} 248.2$, $361.3,228.1$ and 329.2, respectively. Of note, as the MS3 fragment spectrum was used to search against protein database using MASCOT as search engine, the neutral loss of HexNAc in all the MS3 fragment ions had to be defined as a variable modification so that the confidence in the peptide identification and the MASCOT score is increased. 
(A)

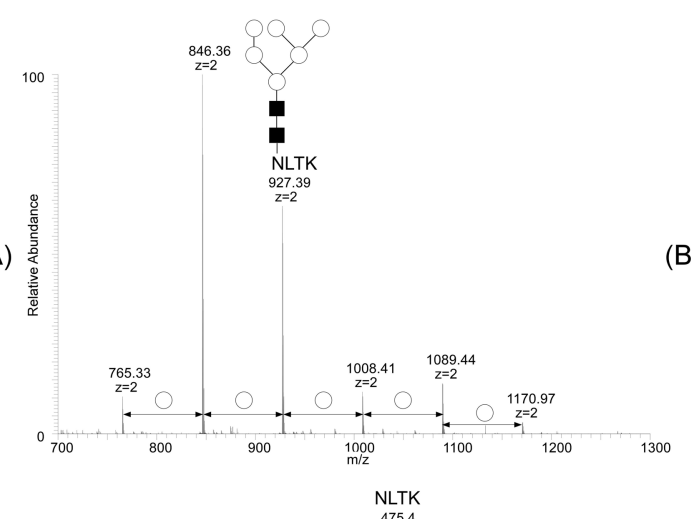

(C)

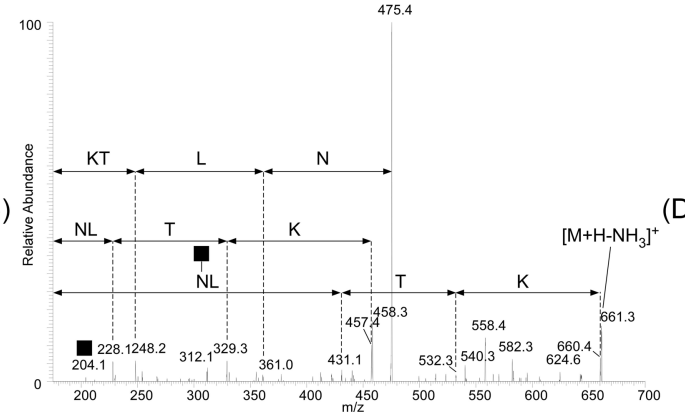

(B)

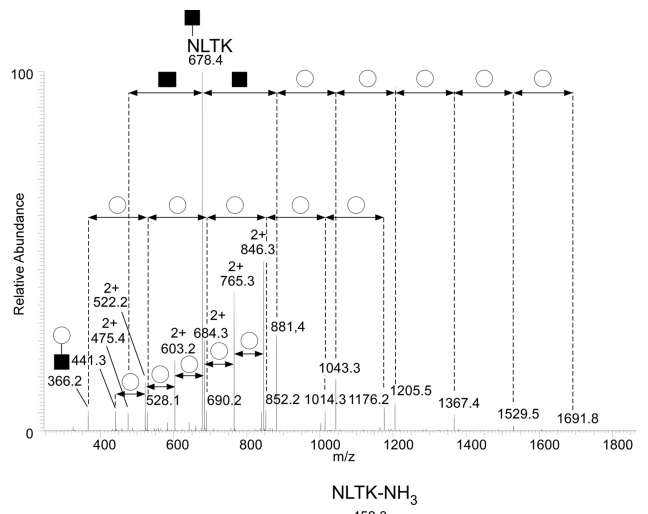

(D)

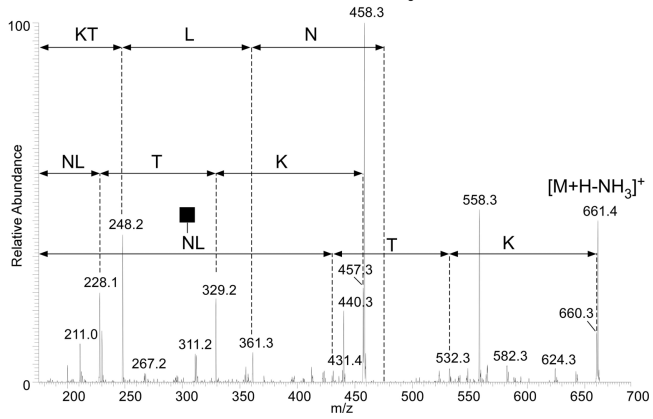

Figure 4.8. Online-nanoLC ESI-Orbitrap coupling with pseudo-neutral-loss scan to identify the tryptic glycopeptide from RNase B. (A) Mass spectrum of tryptic glycosylation peptide ${ }^{34} \mathrm{NLTK}^{37}$ attached to asparagine-34 containing a high-mannose type oligosaccharide. (B) MS/MS spectrum of the tryptic glycopeptide at $m / z 927.4$ triggered by a pseudo-neutral-loss scan for monosaccharide residues. (C) The fragment ion at $\mathrm{m} / \mathrm{z}$ 678.4 (peptide + HexNac) in an MS/MS spectrum was subjected to a second ion isolation/fragmentation cycle to obtain peptide sequence information (targeted normal CID MS3 experiment). (D) Multi-stage activation MS3 corresponding to a neutral loss of HexNAc applied to fragment peptide+HexNAc instead of regular CID MS3. Square, $\mathrm{N}$-acetylglucosamine (HexNAc) and circle, mannose (Man).

\subsection{Conclusion}

We have developed a novel scan function in LTQ-Orbitrap that is highly suitable for the specific detection of phosphorylation at serine and threonine residues and of glycosylation at asparagine residues, and for obtaining sequence information about the peptide moiety. This approach relies on the highly accurate mass spectrometry used here to detect the corresponding mass pairs in the mass scan. The appearance of mass pairs triggers MS/MS and multi-stage activation MS3 to obtain modified peptide sequence information. Nevertheless, there are certain limitations, as in the case of phosphotyrosine the neutral loss of the $\mathrm{H}_{3} \mathrm{PO}_{4}$ moiety is not commonly observed. The list of targeted MS3 for glycopeptide analysis is limited to 10 precursors by Xcalibur software restricting large scale glycoproteomic anlalysis. However, 
we anticipate that this issue could be solved by the ThermoFisherScientific company. We expect this method to be extensively applied for moderately labile PTMs in future, not only for phosphorylation and glycosylation but also for sulphonation, nitrosylation, nitration etc., taking the advantage of CID or even electron-transfer dissociation (ETD) to identify peptide sequence and locate the modification site. 
Chapter 5 - "ChopNSpice", a Mass Spectrometric Approach That Allows Identification of Endogenous Small Ubiquitin-like Modifier-conjugated Peptides

\subsection{Summary}

Post-translational modification by conjugation of small ubiquitin-like modifier (SUMO) to its substrate is emerging as an important protein regulatory mechanism involved in diverse range of cellular processes from yeast to human. Typically, SUMO is covalently conjugated to lysine residues within a SUMO consensus motif ( $\Psi-\mathrm{K}-\mathrm{x}-\mathrm{D} / \mathrm{E}$, where $\Psi$ is a hydrophobic amino acid residue $\mathrm{L}, \mathrm{I}, \mathrm{V}$, or $\mathrm{F}, \mathrm{x}$ is any amino acid residue, and $\mathrm{D}$ or $\mathrm{E}$ is an acidic residue); however, an increasing number of SUMOylated proteins are on non-consensus sites. To appreciate the functional consequences of SUMOylation, the identification of SUMO attachment sites is of critical importance. Discovery of SUMO acceptor sites is usually performed by a laborious mutagenesis approach or using MS. In MS, identification of SUMO acceptor sites in higher eukaryotes is hampered by the large tryptic fragments of SUMO1 and SUMO2/3. Current mass spectrometric (MS)-based protein modification search engines in combination with known databases lack the possibility to search MS/MS spectra for larger modifications, such as SUMOylation. We therefore developed a novel, user-friendly and straightforward database search tool called "ChopNSpice" (http://chopnspice.gwdg.de/). In combination with current proteomic search engines like MASCOT or Sequest, ChopNSpice successfully allows unambiguous identification of mammalian SUMO acceptor sites from proteins SUMOylated in vivo and in vitro. To increase the sensitivity for the experimental detection of SUMOylated peptides, we used high mass MS/MS acquisition conditions in an Orbitrap mass spectrometer. Under these conditions only peptides with masses exceeding $2154 \mathrm{Da}$ (for SUMO 1) or 3568 $\mathrm{Da}$ (for SUMO 2/3) are selected. Our approach is highly suitable for the accurate detection and sequencing of the large SUMOylated peptides and additionally facilitates their detection although they are low abundance. By applying this approach we demonstrated the power of this technique by the identification of the SUMO acceptor sites in, among others, endogenous SUMO1, SUMO2, RanBP2, and Ubc9.

\subsection{Introduction}

\subsubsection{SUMOylation}

Post-translational modification with ubiquitin and ubiquitin-like modifiers (Ubl) such as SUMO play an important role in most - if not all - cellular processes shown in Figure 5.1 [8, 157-161]. Conjugation of Ubls to their targets involves an isopeptide bond between the carboxyl group of the modifier and the $\varepsilon$-amino group of a lysine residue within the targets shown in Figure 5.1. 


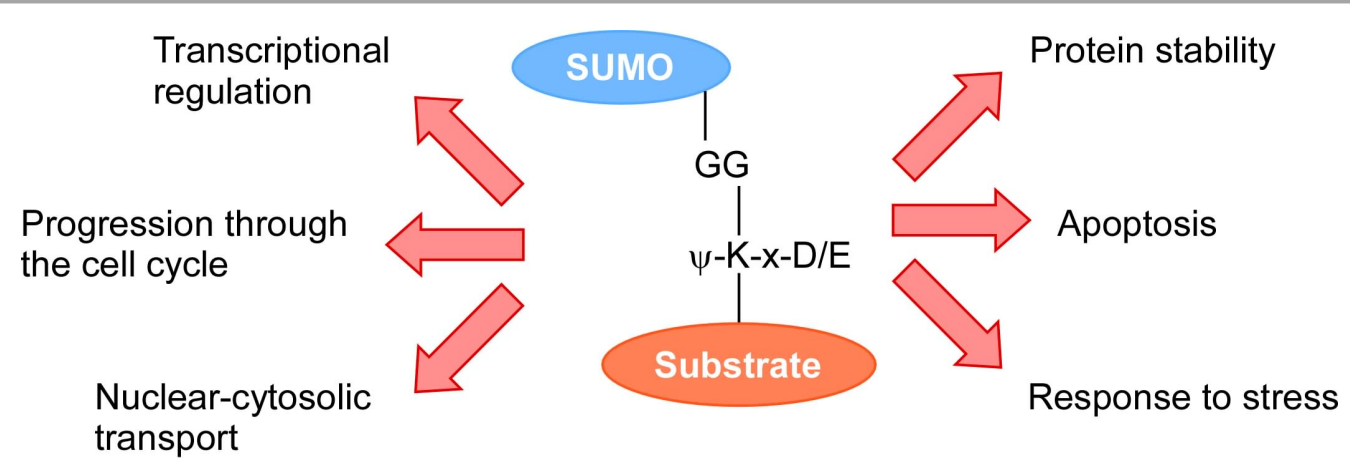

Figure 5.1. Small ubiquitin-like modifier (SUMO). SUMOylation is a covalent post-translational modification located within the consensus motif $\psi-\mathrm{K}-\mathrm{x}-\mathrm{D} / \mathrm{E}$, where $\psi$ represents a large hydrophobic amino acid, and $x$ represents any amino acid. The SUMOylation involves in various cellular processes such as transcriptional regulation, progression through the cell cycle, nuclear-cytosolic transport, protein stability, apoptosis and response to stress.

Attachment of SUMO to specific substrates involves four-step enzymatic pathway analogous to ubiquitylation shown in Figure 5.2 [162].

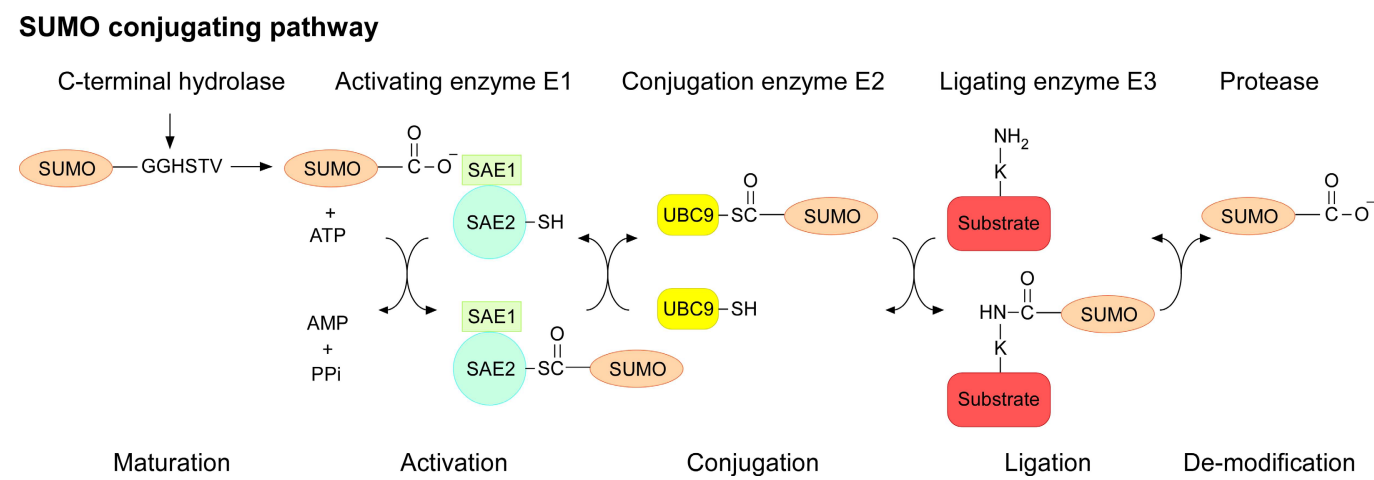

Figure 5.2. SUMO conjugating pathway. The SUMO precursor is processed by a SUMO specific protease to reveal the C-terminal di-glycine and then is activated by the E1 enzyme. After transesterification onto the E2 conjugating enzyme (Ubc9), the SUMO conjugated substrate is selected. The SUMO is ligated to the substrate with the help of an E3 ligating enzyme. SUMOylation is reversible and can be deconjugated from the targeted protein by the action of specific SUMO proteases.

First the SUMO is processed by protease, known as SENP for human and Ulp1 for yeast, to expose their C-terminal di-glycine. Subsequently, the SUMO is conjugated to E1 by ATP-dependent formation of a thioester bond between the C-terminal glycine of SUMO and the SUMO-specific E1 activating enzyme, known as a heterodimeric activating enzyme SAE1/SAE2 and Uba2p/Aos1p for human and yeast, respectively. The activated SUMO 
protein is then transferred thioester bond from the E1 activating enzyme to the E2 conjugating enzyme, Ubc9. The Ubc9 is reported to recognize and bind directly to a consensus SUMOylation motif $\Psi-K-x-D / E$ [163-166]. The E3 ligating enzyme, such as RanBP2 or members of the PIAS family [161, 167], in turn efficiently catalyzes the formation of an isopeptide bond between the C-terminal glycine of SUMO and the $\varepsilon$-amino group of lysine residue in the substrate proteins. The conjugation status undergoes perpetual change, and is governed by a small family of SUMO proteases that hydrolyze the isopeptide bond between SUMO and its target [168, 169]. Unlike yeast and other invertebrates, which is present only one SUMO gene, vertebrates express at least three different SUMO isomers: SUMO1, SUMO2 and SUMO3. Human SUMO2 and SUMO3 (referred to as SUMO2/3) share $86 \%$ sequence identity, but SUMO1 exhibits $44 \%$ sequence identify with SUMO2/3 [161]. Although all SUMO proteins share the conserved ubiquitin domain and utilize the same E1 and E2 enzymes for cleavage/attachment of the C-terminal diglycine of SUMO, the molecular basis for their substrates favorite and the additional recruitment of E3 ligases remain unclear [164, 170]. While the list of known SUMO substrates is growing rapidly, our understanding of the functional consequences for many of these targets is lagging behind. At a molecular level, the functional consequences of SUMO conjugation can be explained by a gain or loss of interaction with other macromolecules [157, 159]. The SUMO-dependent intramolecular conformational changes have also been described [171, 172]. Thus, to appreciate the role that SUMO plays in the regulation of specific substrates, identification of the acceptor site(s) for SUMO conjugation is of key importance. So far, identification of SUMO acceptor sites has relied largely on mutation of the consensus SUMOylation motif $\Psi-\mathrm{K}-\mathrm{x}-\mathrm{D} / \mathrm{E}$. This motif is recognized by Ubc9, if presented in an extended conformation [163-166]. However, an increasing number of protein substrates, such as PCNA, E2-25K, Daxx and USP25, turn out to be SUMOylated on lysine residues that do not consist with the SUMO consensus site [173-176]. For this category of proteins, as well as for proteins that contain a large number of SUMO consensus sites, the identification of acceptor lysines is a burdensome task that often involves mutagenesis of each lysine residue within the substrate in turn.

\subsubsection{Challenges for Identification of SUMOylation by MS-based Approach}

Mass spectrometry (MS) is currently one of the state-of-the-art technologies to identify protein factors and their post-translational modifications in an unbiased and sensitive manner. Several groups have shown that, using over-expressed tagged SUMO, MS can be efficiently exploited to identify endogenous substrates for SUMO conjugation [177-179]. However, the identification of SUMO-acceptor lysines using MS has remained a more challenging task [177, 180-182]. To date, using affinity tagged SUMO technology, unbiased identification of acceptor lysines for endogenous substrates has only been observed in S. cerevisiae [179]. The identification of substrates in higher eukaryotes has been hampered by the large conjugated SUMO peptide 
that arises upon tryptic digestion (> $2154 \mathrm{Da}$ with human SUMO1, >3568 Da with human SUMO2/3; compared with $484 \mathrm{Da}$ for Smt3 in S. cerevisiae). Such large fragments, in addition to the mass of the conjugated peptide, can impede their in-gel digestion, extraction, detection and sequencing in MS. Consequently, MS and MS/MS result in fragment-ion spectra that are too complex to interpret manually. To circumvent these problems, several different strategies have been developed: (1) identification of targets using an in vitro to in vivo approach [180], (2) a mutational SUMO approach yielding a smaller tryptic fragment of SUMO that simplifies the identification of SUMO acceptor sites by MS [181], and (3) development of an automated recognition pattern tool (SUMmOn) [182]. Although these approaches have been applied successfully for the identification of SUMO conjugations in vitro and in vivo, unbiased identification of SUMO conjugations in vivo has not been achieved in higher eukaryotes. Another hurdle to such identification of SUMO conjugations is the variety of masses that can theoretically arise for just one SUMO-conjugated lysine in a given protein due to tryptic mis-cleavages. Thus, the unambiguous identification of SUMO acceptor sites requires the mass of the modified peptide carrying the conjugated SUMO (fragment) to be measured with high accuracy and - most importantly - it requires sequence analysis of the modified peptides. Since available proteomic search engines lack the possibility to search MS/MS spectra for larger modifications, e.g. those that occur upon SUMOylation, we developed a novel, simple and straightforward database search tool (ChopNSpice) that, in combination with current proteomic search engines (such as Sequest [49] or MASCOT [50]), allows one to identify SUMO1 and SUMO2/3 acceptor sites unasmbiguously. We confirmed this strategy in vitro on various substrates, and demonstrate the power of this technique by the identification of acceptor lysines within several endogenous targets from HeLa cells.

\subsection{Experiment Sections}

\subsubsection{Materials}

Ammonium bicarbonate, iodoacetamide (IAA) were obtained from Sigma-Aldrich (St. Louis, $\mathrm{MO}$ ). Sequencing grade, modified trypsin was obtained from Promega (Madison, WI). Dithiothreitol (DTT), formic acid and acetonitrile (ACN) were obtained from Merck (Darmstadt, Germany). Mouse monoclonal $\alpha$-SUMO1 antibodies were kindly provided by M. Matunis and goat anti-SUMO1 antibodies were produced by Prof. Dr. Frauke Melchior's laboratory [183, 184]. Secondary antibodies were obtained from Jackson Laboratories. Plasmids for bacterial expression of Aos1/Uba2, Ubc9, SUMO1, SUMO2 (NM_006937), GST-SP100, RanGAP1, were produced by Prof. Dr. Frauke Melchior's laboratory [176, 185]. A plasmid for GST-p53 was kindly provided by Dr. Moshe Oren. Recombinant protein purification for SUMO1, SUMO2, Aos1/Uba2, Ubc9, RanGAP1, GST-SP100, GST-p53 and PIAS1 were produced by Prof. Dr. Frauke Melchior's laboratory [176, 185-187]. 


\subsubsection{Software}

ChopNSpice was written in PHP by Benedikt Frank. The software tool is freely available online (http://chopnspice.gwdg.de/), and also releases as open source under the terms of the General Public License v3 (GPLv3).

\subsubsection{In vitro SUMOylation Assays}

The in vitro SUMOylation assays were performed by Dr. Erik Meulmeester. SUMO conjugation reactions were performed at $30{ }^{\circ} \mathrm{C}$ for 1 hour in the presence or absence of $5 \mathrm{mM}$ ATP in 20 $\mu \mathrm{L}$ transport buffer TB $(20 \mathrm{mM}$ Hepes $/ \mathrm{KOH} \mathrm{pH} 7.3,110 \mathrm{mM}$ potassium acetate, $2 \mathrm{mM}$ magnesium acetate, $0.5 \mathrm{mM}$ EGTA, $1 \mathrm{mM}$ DTT supplemented with protease inhibitors). Reactions contained $100 \mathrm{ng}$ Aos1/Uba2, $200 \mathrm{ng}$ Ubc9, $2.5 \mu \mathrm{g}$ SUMO1 or SUMO2 and $1 \mu \mathrm{g}$ target protein (GST-p53, mouse RanGAP1, GST-SP100 or Aos1/Uba2) in a volume of $20 \mu \mathrm{L}$.

\subsubsection{Cell culture, Immunoprecipitation and Immunoblotting}

Cell culture, immunoprecipitation and immunoblotting experiments were performed by Dr. Erik Meulmeester. Hela-S3 cells were maintained in Jokliks medium supplemented with $10 \%$ fetal bovine serum and antibiotics. To immunoprecipitate SUMO1 conjugates, $1 \times 10^{8}$ HeLa cells were washed twice with PBS containing $10 \mathrm{mM}$ NEM and lysed in two pellet volumes RIPA buffer (20 mM NaP $(\mathrm{pH} 7.4), 150 \mathrm{mM} \mathrm{NaCl}, 1 \%$ Triton, $0.5 \%$ sodium deoxycholate, $0.1 \%$ SDS) supplemented with protease inhibitors and $10 \mathrm{mM}$ NEM. Lysates were centrifuged (16.000 g for $15 \mathrm{~min}$. at $4{ }^{\circ} \mathrm{C}$ ) and filtered $(0.45 \mu \mathrm{m})$ prior to addition of $25 \mu \mathrm{g}$ monoclonal $\alpha$-SUMO1 antibodies. After 2 hours incubation at $4{ }^{\circ} \mathrm{C}$, the lysates were centrifuged $(16.000 \mathrm{~g}$ for $15 \mathrm{~min}$. at $4{ }^{\circ} \mathrm{C}$ ) and the supernatant was incubated for another 2 hours at $4{ }^{\circ} \mathrm{C}$ with protein $\mathrm{G}$ agarose. After collection and extensive washing of bound proteins, samples were eluted with $2 x$ sample buffer and separated on SDS-PAGE followed by Coomassie staining or western blotting. In a second larger experiment using $1 \times 10^{9}$ cells were lysed in TB $(0,1 \%$ triton, $10 \mathrm{mM}$ ATP) and were treated with $10 \mathrm{mM}$ NEM after lysis. Immuno-precipitation was similar as described above, using $100 \mu \mathrm{g}$ GMP1 antibodies. The SUMO acceptor site in RanGAP1 was observed in both purification methods while the other targets were identified in the second up scaled experiment.

\subsubsection{Protein Digestion}

SUMO-conjugated proteins were excised from gel, reduced with $50 \mathrm{mM}$ DTT for 1 hour, alkylated with 100 mM IAA for 1 hour and in-gel digested with modified trypsin (Promega) overnight, all at $37{ }^{\circ} \mathrm{C}$. SUMO-conjugated proteins from solution were reduced with $50 \mathrm{mM}$ DTT for 1 hour, alkylated with 100 mM IAA for 1 hour and subsequently digested with modified trypsin overnight, all at $37{ }^{\circ} \mathrm{C}$. 


\subsubsection{Liquid Chromatography and Mass Spectrometry}

The resulting tryptic peptides were dissolved in $2 \mu \mathrm{L} 50 \%$ ACN with $0.1 \%$ formic acid and added to $18 \mu \mathrm{L} 0.1 \%$ formic acid for further MS analysis. MS analysis was performed by nanoscale liquid chromatography-tandem mass spectrometry (LC-MS/MS) using a LTQ-Orbitrap mass spectrometer (Thermo Fisher Scientific) equipped with a nanoelectrospray ion source and coupled to an Agilent $1100 \mathrm{HPLC}$ system (Agilent Technologies), fitted with an self made $\mathrm{C} 18$ column. Tryptic peptides were first loaded at a flow rate of $10 \mu \mathrm{L} / \mathrm{min}$ onto a C18 trap column (1.5 cm, $360 \mu$ m o.d., $150 \mu$ i.d., Reprosil-Pur $120 \AA, 5 \mu \mathrm{m}, \mathrm{C} 18-A Q$, Dr. Maisch $\mathrm{GmbH}$, Germany). Retained peptides were eluted and separated on an analytical $\mathrm{C} 18$ capillary column (15 cm, $360 \mu \mathrm{m}$ o.d., $75 \mu \mathrm{m}$ i.d., Reprosil-Pur $120 \AA, 5 \mu \mathrm{m}, \mathrm{C} 18-\mathrm{AQ}$, Dr. Maisch $\mathrm{GmbH}$, Germany) at a flow rate of $300 \mathrm{~nL} / \mathrm{min}$, with a gradient from 7.5 to $37.5 \% \mathrm{ACN}$ in $0.1 \%$ formic acid for $60 \mathrm{~min}$. Typical MS conditions were: spray voltage, $1.8 \mathrm{kV}$; heated capillary temperature, $150{ }^{\circ} \mathrm{C}$; normalized collision-induced dissociation (CID) collision energy $37.5 \%$ for MS/MS in LTQ. An activation q $=0.25$ and activation time of $30 \mathrm{~ms}$ were used. The mass spectrometer was operated in the data dependent mode to automatically switch between MS and MS/MS acquisition. Survey full scan MS spectra (from $\mathrm{m} / \mathrm{z} 350-2000$ ) were acquired in the Orbitrap with resolution $R=30,000$ at $\mathrm{m} / \mathrm{z} 400$ (after accumulation to a 'target value' of $1,000,000$ in the Orbitrap). The five most intense ions were isolated sequentially and fragmented in the linear ion trap using CID at a target value of 100,000. For all measurements with the Orbitrap detector a lock-mass ion from ambient air ( $\mathrm{m} / \mathrm{z} 445.120025)$ was used for internal calibration. For high-mass data-dependent mode, the mass range for selecting MS data dependent masses was 2154-1000000 and 3568-1000000 for SUMO1 and SUMO2/3, respectively, and used $\mathrm{m} / \mathrm{z}$ value as masses.

\subsubsection{Data Analysis}

For protein identification, all MS/MS spectra were searched against a Swiss-Prot database using MASCOT with the following parameters: mass tolerance of $10 \mathrm{ppm}$ in MS mode and 0.8 $\mathrm{Da}$ in MS/MS mode; allow up to two missed cleavage; variable modifications considered were methionine oxidation and cysteine carboxyamidomethylation. The sequence of the protein of interest was manually saved to a FASTA file and ChopNSpice was used to create a new FASTA file with the following parameters: spice species was $\mathrm{H}$. sapiens; spice sequences were SUMO1 and SUMO2, respectively; spice site was KX; spice mode was once per fragment; include unmodified fragments in output; enzyme was trypsin K/R, don't cleave at $\mathrm{P}$; allowed up to three protein miscleavages; allowed up to one miscleavage in the "spice sequence"; output formatting was FASTA: single protein sequence; marked all cleaved site $(\mathrm{J})$; retain comments in FASTA format; without line breaks in FASTA output. For SUMOylated site identification with MASCOT or Sequest, all MS/MS spectra were searched against a new FASTA file which was 
created by ChopNSpice with the following parameters: mass tolerance of $10 \mathrm{ppm}$ in MS mode and $0.8 \mathrm{Da}$ in MS/MS mode; allow zero missed cleavage; variable modifications considered were methionine oxidation and cysteine carboxyamidomethylation; enzyme was cleavage $\mathrm{J}$ at $\mathrm{N}$ and $\mathrm{C}$ terminus for MASCOT or no enzyme must be used for Sequest. If the search was performed with the in-house MASCOT server, the file "quant_subs.pl" must be changed from ' $\mathrm{J}$ ' => 0 to ' $\mathrm{J}$ ' => 0.05 in line 3653). All MS/MS spectra were confirmed manually to identify the SUMO acceptor site. The symbol of the amino acid which was before and after the identified SUMO-conjugated peptide must be J. All high-abundance peaks had to be assigned to $y$ - or bion series).

\subsection{Result and Discussion}

\subsubsection{ChopNspice}

A typical strategy in MS-based proteomics comprises enzymatic digestion of proteins with endoproteinases, separation of the resulting peptides by liquid chromatography (LC), and ionization and subsequent fragmentation of the peptides. Afterward the automated search of the fragment spectra against a database allows identification of the corresponding protein [35]. In addition, the identification of post-translational modifications by MS requires a highly accurate mass determination of the precursor and a sequence information of the peptide containing the modification. Search engines such as MASCOT and/or SEQUEST are commonly used among the MS-based proteomic researcher, and the output format of these search engines are widely accepted in the community. We developed a software tool "ChopNSpice" that makes use of these search engines, implements new modified protein sequences to the standard databases and allows to identity modified sites.

Accordingly, our approach to identify SUMO acceptor sites is based on the fragmentation pattern of the SUMO-conjugated peptides, which are digested with trypsin. Such digestion results in the peptide in which a missed (i.e., non-cleaved, owing to SUMO modification) lysine residue is branched with a SUMO tryptic peptide shown in Figure 5.3A. In practice, we and others observed that the spectrum of MS/MS fragmentation pattern of such a branched peptide is similar to a tryptic linear peptide that has a miscleaved lysine residue and the SUMO peptide at its $\mathrm{N}$-terminus shown in Figure $5.3[180,188]$. Identification of SUMO acceptor lysines using such MS/MS spectra in a database search is only possible when the peptide sequences within the database are also modified by SUMO sequence. However, available protein database search engines for experimental fragment spectra do not include SUMO sequence as a putative modification at lysine residues. Simple addition of the molecular weight of the tryptic SUMO peptide to that of a lysine residue within the targeted protein, without obtaining sequence information, would create a large number of false positive hits in database searches. In addition, since protein SUMOylation can theoretically occur at every lysine 
residue within a substrate protein, manual construction of such artificial peptides, calculation of molecular weight and matching MS/MS fragment pattern are time-consuming processes.
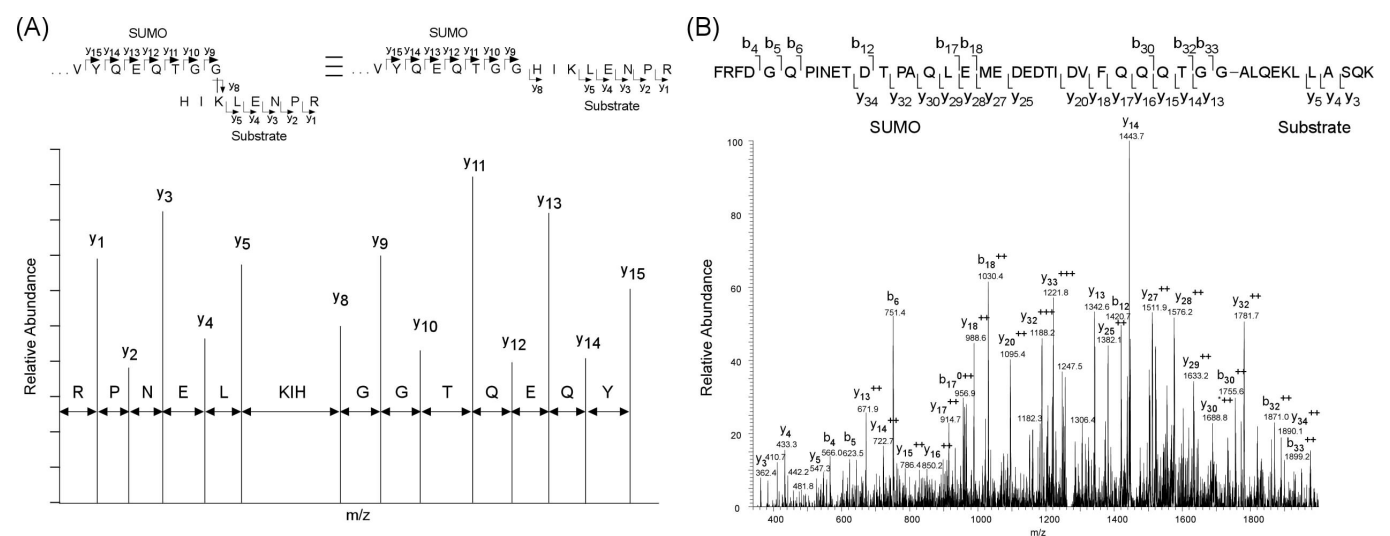

Figure 5.3. The fragment pattern of SUMO-conjugated peptide similar to linear peptide. (A) Branched tryptic peptide conjugated with tryptic SUMO fragment at its lysine acceptor site reveal a similar MS/MS fragmentation pattern as a linear peptide. The y-type ions in the artificial spectrum and in the peptide sequence are indicated. (B) The CID MS/MS spectrum of a tryptic SUMO-conjugated peptide recorded on an Orbitrap mass spectrometer. The Figure depicts the tryptic fragment of USP25 (encompassing positions 711-721) conjugated with SUMO2. The y-type ions in the MS/MS spectrum and in the peptide sequence are indicated.

Accordingly, we developed an algorithm to automatically generate such SUMO-conjugated FASTA sequences of proteins in silico shown in Figure 5.4A. Subsequently, the novel FASTA sequences are implemented in commonly used database search engines to identify lysine acceptor sites for SUMO conjugation shown in Figure 5.4B. More specifically, the FASTA sequence of a putatively SUMOylated protein is "chopped" into tryptic fragments (allowing 0, 1, 2 or n missed cleavages). The tryptic "spice" sequence (e.g. tryptic peptides from SUMO1 or any other ubiquitin-like protein) is attached to the $\mathrm{N}$-terminus of each tryptic peptide that contains a lysine $(\mathrm{K})$ as a missed cleavage site. It is of note that also the ubiquitin-like proteins are allowed to contain $0,1,2$ or $n$ miscleavage(s). To prevent the appearance of non-natural peptides, a virtual amino acid " $\mathrm{J}$ " is attached to the $\mathrm{C}$-terminus of each tryptic fragment before ligation of the generated tryptic fragments into one large FASTA sequence. This large artificial protein sequence is submitted into the database search engine in which the virtual cleavage site "J" is recognized by an artificial endoproteinase that directly cleaves $\mathrm{N}$ - and C-terminally to "J" to generate the tryptic fragments for the selected missed cleavages. Subsequently the SUMO acceptor site can be identified by using the applied search engine (e.g. MASCOT, X!Tandem or Sequest). A workflow to set up a modified FASTA sequence in which certain 
proteins (or entire databases) can be generated by a user-defined modifier is implemented in the program ChopNSpice (http://chopnspice.gwdg.de/).

(A)

Initial protein sequence

$x x K x x R \operatorname{Rx} x x K x x x x x R x x R x x x K x x x x$

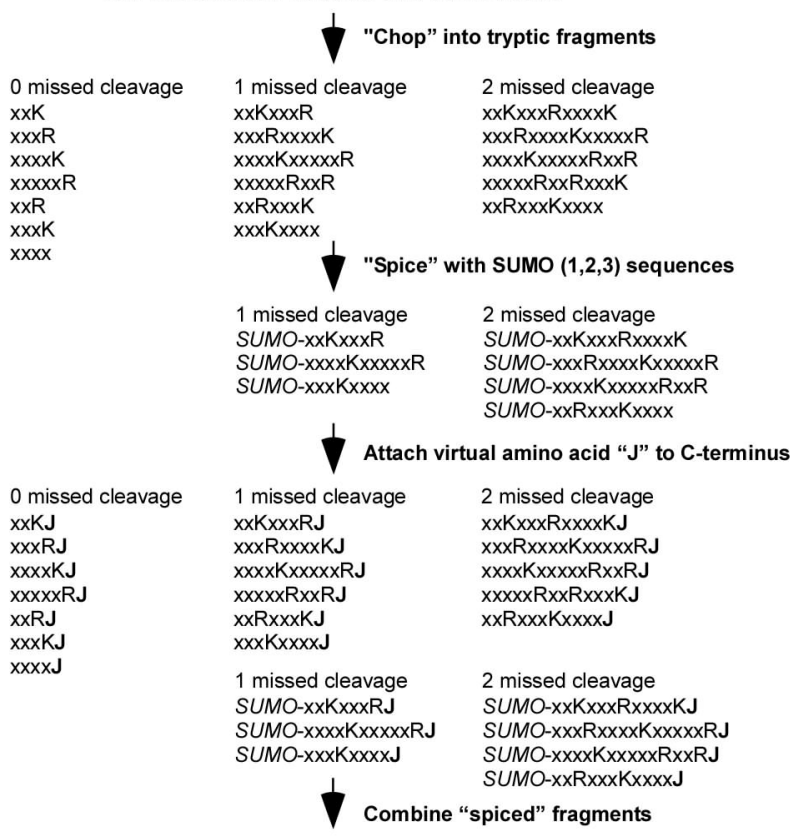

"Spiced" protein sequence

$x x K J x x x R J x x x x K J x x x x x R J x x R J x x x K J J x x x J J x x K x x x R J x x x R x x x x K J x x x x K x x x x x$ RJ $x x x x x R x x R J x x R x x x K J x x x K x x x x J x x K x x x R x x x x K J x x x R x x x x K x x x x \times R J x x x x K$

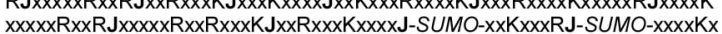

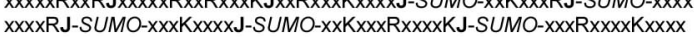

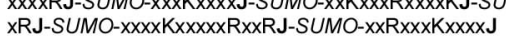

(B)

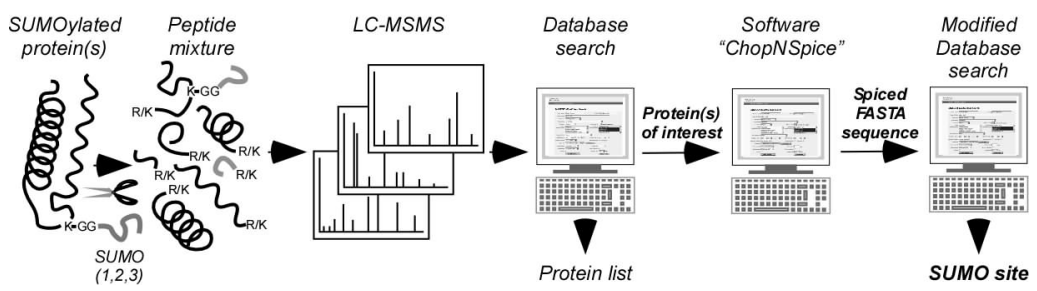

Figure 5.4. The concept of the "ChopNSpice" software. (A) The basic workflow of "ChopNSpice" to generate a "spiced" FASTA sequence from an initial protein sequence in which all lysine residues are putatively modified by SUMO1 or SUMO2/3. The "spiced" FASTA sequence is subsequently used in database searches. (B) The general workflow for identification of SUMO acceptor sites. Sumoylated proteins are digested with endoproteinases and analyzed by LC-MS/MS. The corresponding proteins are identified by a database search engines (MASCOT and/or Sequest). Putatively sumoylated protein sequences are "chopped and spiced" (see A) and the "spiced" FASTA sequences are added to the database. The protein database search is repeated to identify the SUMOconjugated peptide with its corresponding lysine acceptor site (see text for details). 
In practical terms, after enrichment of endogenous SUMO-conjugated proteins or proteins SUMOylated in vitro, putative SUMO substrates are identified by a standard MS-based protein identification, i.e. samples are digested with trypsin and the resulting tryptic peptides are separated by nano-LC, detected and sequenced in the MS. Corresponding proteins in the sample are identified by highly accurate mass of the peptide and matching the fragment spectra against a protein database using e.g. MASCOT, X!Tandem or Sequest as search engines. A second MS and MS/MS analysis under "high mass" conditions is performed where only those precursors exceed a certain mass, i.e. $\geq 2154$ Da for SUMO-1 and $\geq 3568$ Da for SUMO-2/3, are selected for sequencing. Once one or several putatively SUMOylated proteins have been identified in both the analyses after merging the data/results, MS and MS/MS data are resubmitted to search against the new database containing the virtual SUMOylated protein sequence generated by ChopNSpice (Figure 5.4B). In a subsequent experiment, the same sample can be reinvestigated by extended/modified LC-MS/MS analysis to identify the SUMO acceptor site(s).

Note that both of the search engines used in this study (MASCOT and Sequest) have some shortcomings. For instance, MASCOT does not efficiently search MS/MS fragment spectra that contain fragment ions with a charge state higher than 2; as a consequence, larger SUMOylated peptides with quadruply or higher charge states show a very low score in MASCOT result or are not identified at all. This problem can be circumvented by using either Sequest or other search engines (e.g. X!Tandem) or, alternatively, by using the software tool Raw2msn to deconvolute the higher charge stages of the fragment ions in the raw data to singly-charged fragment ions for MASCOT search [189]. However, a prerequisite for deconvolution process is that MS/MS spectra (generated either by CID or by HCD) are recorded in the Orbitrap analyzer with sufficient mass resolution for the charge state recognition; however, this in turn decreases sensitivity [190]. A comparison between the different systems for processing raw data and the different detection modes of the Orbitrap MS are shown in supplementary Figure 5.5. "Sequest" on the other hand does not allow for cleavage with endoproteinase "J" both $\mathrm{N}$ - and C-terminally to $\mathrm{J}$, but rather either $\mathrm{N}$-terminally or C-terminally. Therefore, cleavage of the FASTA sequence is performed unspecifically, i.e., no enzyme is used in silico and matched spectra are validated manually. Confidence in the results from the search engine is achieved by the high mass accuracy of the Orbitrap instrument $(<10 \mathrm{ppm})$, and by the fact that the validated sequence must be preceded or followed by the virtual amino acid J. Furthermore, all the abundant fragment ions must be assigned to $y$ - and/or b-ion series. However, as a very simple alternative, the single concatenated peptide sequences can be submitted to the database without merging them into a single new FASTA sequence. 

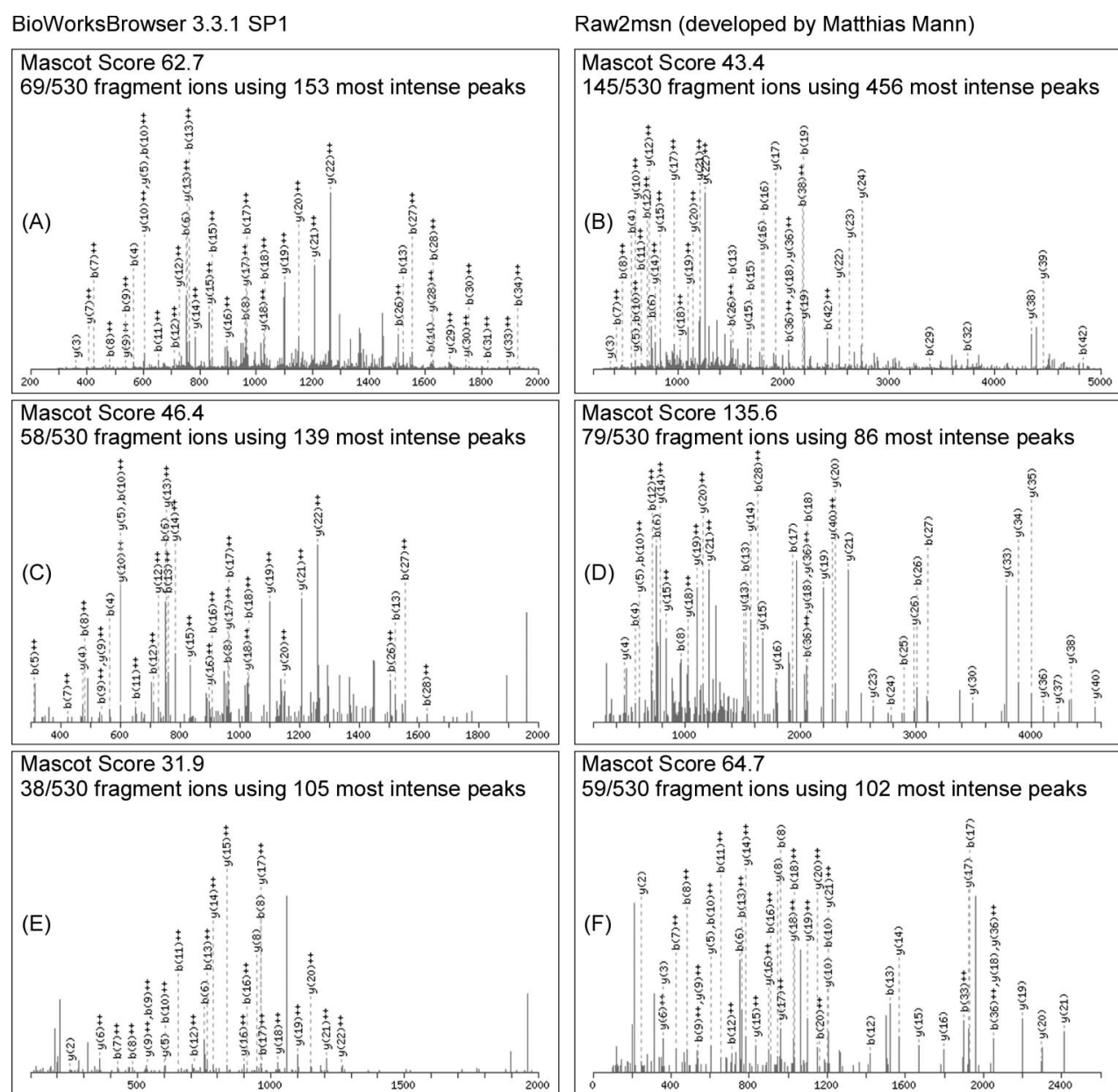

Figure 5.5. MS/MS spectra of USP25 peptide conjugated to SUMO2 at Lysine-754 (M.W.= 5304.5409, 5+, FRFDDQPINETDTPAQLEMEDEDTIDVFQQQTGG-HLKEEETIQIITK). Left panels (A, C, E): Raw data processed with BioWorksBrowser (ThermoFisherScientific, mgf.file). Right panels (B, D, F): Raw data processed with Raw2msm (msm file - deisotope and deconvolute multiply charge to singly charged state). The software tool is available at: http://www.biochem.mpg.de/mann/publications/2006/0510_01/0510_01.html). (A) and (B) are collision-induced dissociation (CID) MS/MS scanning in the linear ion trap (LTQ) that show high sensitivity but a low resolution and additionally a low mass cut off. (C) and (D) are CID MS/MS scanning in the Orbitrap analyzer that shows a medium sensitivity but a high resolution but still a low mass cut off. $(E)$ and $(F)$ are high collision dissociation (HCD) scanning in the Orbitrap analyzer that have low sensitivity but high resolution and do not show any low mass cut off. The comparison of (D) and (C) and (F) and (E) reveals that deconvoluted spectra match to more $y$ - and b-type ions in the MASCOT database search, obtaining high score. Since MASCOT does not support efficient match of MS/MS fragment spectra derived from quadruply and higher charge states, the use of Raw2msm is a pre-requisite for the comprehensive identification of SUMO-conjugated peptides recorded by Orbitrap analyzer. 


\subsubsection{Identification of SUMO Conjugation Sites in vitro}

To validate our approach we applied Ran GTPase-activating protein 1 (RanGAP1), Nuclear autoantigen Sp-100 (Sp100), Cellular tumor antigen p53 (p53) and SUMO-activating enzyme subunit 2 (uba2) to an in vitro SUMOylation reaction with SUMO1 and SUMO2. Proteins migrating on SDS-PAGE with a higher apparent molecular weight than the original proteins were considered to be SUMOylated (Figure 5.6) and were then processed by nanoLC-MS/MS analysis as described above.

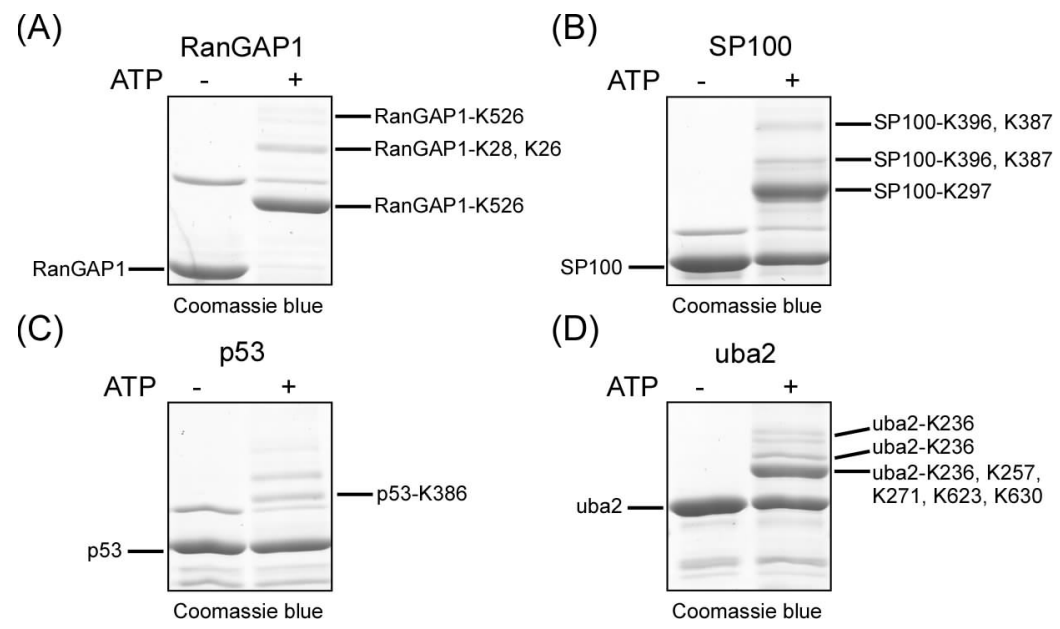

Figure 5.6. Detection of SUMO1-acceptor sites from in vitro sumoylated proteins. (A) In vitro sumoylation of $1 \mu \mathrm{g}$ RanGAP1 with $100 \mathrm{ng}$ Aos1/Uba2, $200 \mathrm{ng}$ Ubc9, $2.5 \mu \mathrm{g}$ SUMO1 for 1 hour at $30{ }^{\circ} \mathrm{C}$. Proteins were visualized by Coomassie blue staining. (B) In vitro sumoylation of $1 \mu \mathrm{g} \mathrm{Sp100} \mathrm{as} \mathrm{in} \mathrm{(A).} \mathrm{(C)} \mathrm{In} \mathrm{vitro} \mathrm{sumoylation} \mathrm{of} 1 \mu \mathrm{g}$ GST-p53 as in (A). (D) In vitro sumoylation of $1 \mu \mathrm{g}$ Aos1-Uba2 as in (A). The acceptor sites identified are indicated at the protein band from which they were discovered.

For identification of SUMO-conjugated peptides, we first tested SUMO as a variable modification on lysines (2154 Da for SUMO 1; $3568 \mathrm{Da}$ for SUMO2) using two commonly used protein identification tools MASCOT and Sequest. Like other groups [182], we were unable to identify any SUMOylated peptides by the standard nanoLC-MS proteomic procedure and subsequent database search. Whereas these search engines rely on the database that contain putative protein sequences and the extra mass added to a particular amino acid for PTMs identification. However, it is of the utmost importance to obtain sequence information not only from the substrate peptide but also from the modifier. Although manual identification of SUMO-conjugated sites was possible, it required laborious searching in the MS spectra for modified peptides [176]. In contrast, by using the ChopNSpice software on the identified protein sequences and subsequent database search with MASCOT and Sequest, we readily identified SUMO modification of RanGAP1 on lysine 526, of p53 on lysine 386 and of Sp100 
on lysine 297. In addition, we observed several minor acceptor sites, also observed by others [191-193]. Furthermore, we discovered that numerous, so far unidentified, lysine residues within the SUMO E1 activating enzyme Uba2 are conjugated with SUMO1 and SUMO2 shown in Table 5.1. However, consistent with the identification of multiple acceptor sites, mutations of single lysine residues within Uba2 did not significantly impair its SUMOylation.

Table 5.1 The in vitro sumoylation assays of RanGap1, SP100, p53 and uba2. The sequence of the SUMOylated peptide and the positions of SUMO-acceptor sites as determined by MS and MS/MS using "ChopNSpice" in combination with MASCOT and SEQUEST as search engines are listed.

\begin{tabular}{|c|c|c|c|c|c|}
\hline Modification & $\begin{array}{c}\text { Protein } \\
\text { Name }\end{array}$ & $\begin{array}{c}\text { MASCOT } \\
\text { Score }\end{array}$ & $\begin{array}{c}\text { Sequest } \\
\text { XCorr }\end{array}$ & Sequence & Site \\
\hline \multirow[t]{12}{*}{ SUMO1 } & RanGap1 & 110.29 & 6.45 & ELGM $_{o x}$ EEEDVIEVYQEQTGG-LLIHM ${ }_{o x}$ GLLKSSEDK & K526 \\
\hline & & 61.9 & 4.81 & ELGM $_{\mathrm{ox}}$ EEEDVIEVYQEQTGG-GKKGLK & K28 \\
\hline & & 72.02 & 5.50 & ELGM $_{\mathrm{ox}}$ EEEDVIEVYQEQTGG-TQVAGGQLSFKKGK & K26 \\
\hline & GST-SP100 & 57.22 & 5.08 & ELGM $_{o x} E E E D V I E V Y Q E Q T G G-L V D I K K K$ & K297 \\
\hline & & 50.53 & 5.40 & ELGMEEEDVIEVYQEQTGG-ESFKKK & K396 \\
\hline & & 70.11 & 4.90 & ELGMEEEDVIEVYQEQTGG-ㅌLSTFR & K387 \\
\hline & p53 & 87.38 & 6.22 & ELGMEEEDVIEVYQEQTGG-HM ${ }_{\text {ox }}$ FKTEGPDSD & K386 \\
\hline & uba2 & 99.43 & 5.97 & ELGMEEEDVIEVYQEQTGG-ENLSAKKR & K623 \\
\hline & & 46.63 & 4.25 & ELGM $_{o x}$ EEEDVIEVYQEQTGG-LFTKLLFK & K257 \\
\hline & & 160.71 & 7.12 & ELGMEEEDVIEVYQEQTGG-ASNEDGDIKRR & K236 \\
\hline & & 124.39 & 7.65 & 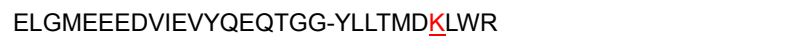 & K271 \\
\hline & & 41.8 & 3.80 & 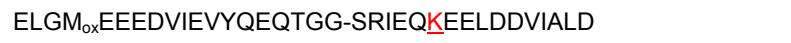 & K630 \\
\hline \multirow[t]{12}{*}{ SUMO2 } & RanGap1 & 118.17 & 8.27 & FDGQPINETDTPAQLEMEDEDTIDVFQQQTGG-LLIHMGLLKKSEDK & K526 \\
\hline & YFP-SP100 & 121.98 & 7.94 & FDGQPINETDTPAQLEM & K297 \\
\hline & & 80.63 & 6.97 & 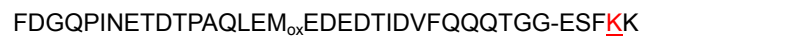 & K396 \\
\hline & & 136.33 & 7.16 & 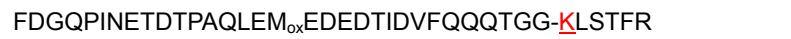 & K387 \\
\hline & & 77.83 & 6.56 & FRFDGQPINETDTPAQLEM ${ }_{\text {ox }} E D E D T I D V F Q Q Q T G G-S \underline{K H G E K ~}$ & K426 \\
\hline & GST-SP100 & 85.66 & 6.56 & FDGQPINETDTPAQLEMEDEDTIDVFQQQTGG-ESF $\underline{K K}$ & K396 \\
\hline & & 132.34 & 7.25 & FDGQPINETDTPAQLEMEDEDTIDVFQQQTGG-ㅌLSTFR & K387 \\
\hline & p53 & 110.79 & 6.81 & FDGQPINETDTPAQLEMEDEDTIDVFQQQTGG-HMFKKTEGPDSD & K386 \\
\hline & uba2 & 152.29 & 9.75 & FRFDGQPINETDTPAQLEMEDEDTIDVFQQQTGG-ASNEDGDIKR & $\mathrm{K} 236$ \\
\hline & & 45.79 & 5.77 & 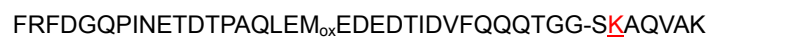 & K72 \\
\hline & SUMO2 & 73.74 & 6.74 & FDGQPINETDTPAQLEM ${ }_{\text {ox }}$ EDEDTIDVFQQQTGG-GSHM ${ }_{\text {ox }}$ ADEKKPK & $\mathrm{K} 5$ \\
\hline & & 85.81 & 7.98 & 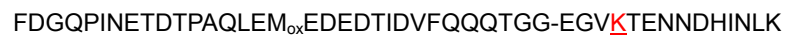 & K11 \\
\hline
\end{tabular}




\subsubsection{Increasing Sensitivity by using "High Mass" Acquisition}

In earlier cooperation with Prof. Dr. Frauke Melchior's laboratory, we mapped two SUMO-conjugated sites within ubiquitin carboxyl-terminal hydrolase 25 (USP25) by which we identified lysine 141 using a mutagenesis approach while the other lysine 99 was identified using MS approach. It is of note that in our previous study we used a small fragment of USP25 that was conjugated with SUMO2 in bacteria followed by purification by gel-filtration and anion-exchange chromatography [176]. However, manual examination of full-length USP25 SUMOylated in vitro did not reveal any SUMO acceptor site. To test whether our ChopNSpice method has an increased sensitivity to identify the acceptor sites of this more complex sample, we conjugated full-length USP25 with SUMO2 in vitro, using the E3 ligase PiasX $\alpha$, as described previously [176]. Next, the mixture was digested with trypsin in solution. Subsequently, to increase sensitivity for the identification of SUMO acceptor sites, we also used "high mass" MS/MS acquisition conditions (Figure 5.7A, compare the standard - upper panel - with the high mass - lower panel). Under these conditions, only peptides with a mass exceeding $2154 \mathrm{Da}$ (for SUMO 1) or $3568 \mathrm{Da}$ (for SUMO 2/3) are selected for MS/MS acquisition.

(A)

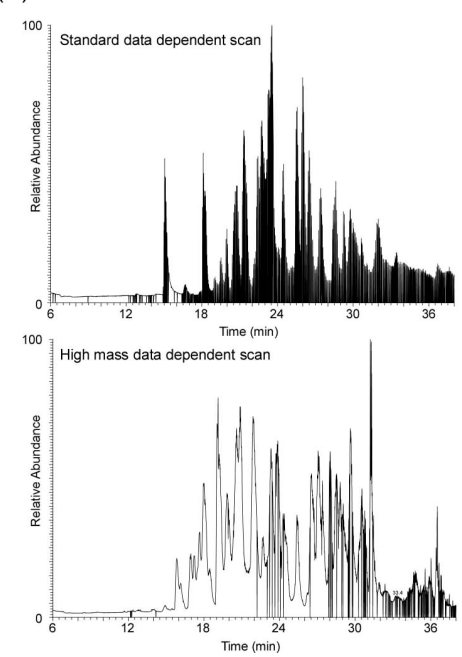

(B)

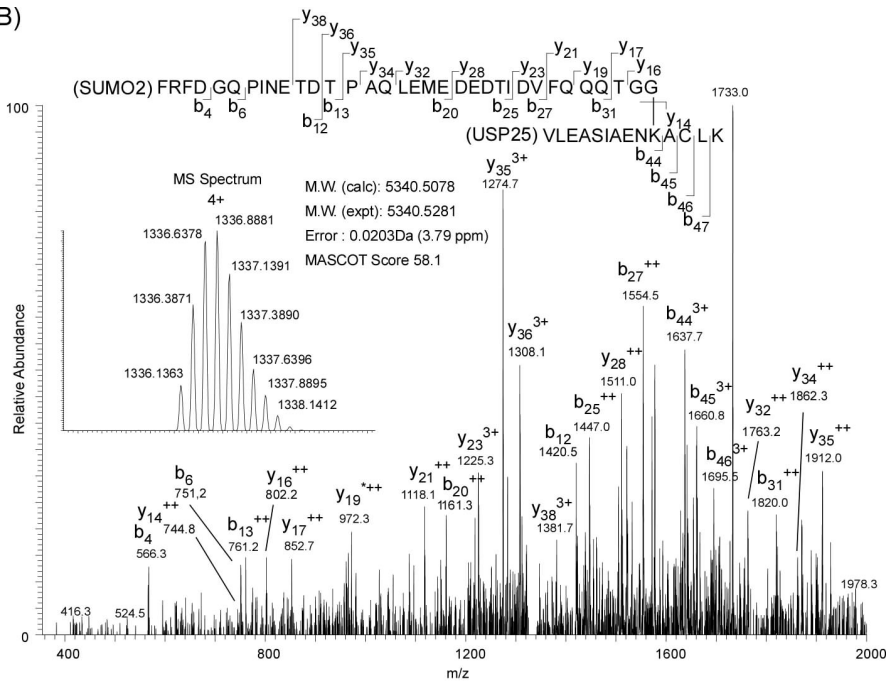

Figure 5.7. Increased sensitivity to discover SUMO acceptor sites. (A) Comparison of the Total Ion Count (TIC) under standard conditions (upper panel) with the TIC under "high mass" conditions (lower panel). The black lines indicate the MS/MS experiment performed. (B) MS/MS CID spectrum of a tryptic peptide $(\mathrm{m} / \mathrm{z}=1336.1363)$ derived from USP25 encompassing positions 132-145 with fragment ions recorded in the LTQ-Orbitrap MS. MS/MS in combination with database search of the modified USP25 sequence (using ChopNSpice) identified Lys-141 as actual SUMO site. Y- and b-type ions are shown in the spectrum and at their respective positions in the conjugated peptide. It is of note that Lys 141 was identified under the high mass conditions only. 
This approach is highly suitable for the accurate detection and sequencing of larger peptides and additionally facilitates detection of lower-abundance SUMO conjugations. A database search against modified sequences (achieved by the program ChopNSpice in combination with MASCOT) demonstrated that SUMOylated peptides were enriched by high-mass MS/MS acquisition shown in Table 5.2. Using this strategy, we went on to identify several additional SUMO acceptor sites within full-length USP25, including lysine 141, which had previously been identified only by a mutational approach. In addition, we observed lysine 5 in SUMO2 as an acceptor site for chain formation, consistent with a previous report [180].

Table 5.2. The in vitro sumoylation assays of USP25 by using either standard data dependent acquisition (DDA) or high mass data dependent acquisition in the LTQ-Orbitrap MS. The sequence of the SUMOylated peptide and the positions of SUMO-acceptor sites as determined by MS and MS/MS using "ChopNSpice" in combination with MASCOT and SEQUEST as search engines are listed.

\begin{tabular}{|c|c|c|c|c|c|c|}
\hline $\begin{array}{l}\text { Protein } \\
\text { Name }\end{array}$ & $\begin{array}{l}\text { Mascot } \\
\text { Score }\end{array}$ & $\begin{array}{l}\text { Sequest } \\
\text { XCorr }\end{array}$ & Sequence & Site & $\begin{array}{c}\text { Stadnard } \\
\text { DDA }\end{array}$ & $\begin{array}{c}\text { High M.W. } \\
\text { DDA }\end{array}$ \\
\hline \multirow[t]{8}{*}{ USP25 } & 181.95 & 8.62 & ACLKR & K145 & & $\mathrm{X}$ \\
\hline & 87.73 & 7.89 & NQKEEERR & K214 & & $X$ \\
\hline & 187.12 & 8.89 & LLASQKLLR & K721 & $x$ & $x$ \\
\hline & 128.21 & 9.48 & ALQEKLLLASQK & K715 & & $\mathrm{X}$ \\
\hline & 75.48 & 8.18 & SVIHKEFTQSR & K520 & & $x$ \\
\hline & 135.63 & 7.99 & HLKEEETIQIITK & K754 & & $x$ \\
\hline & 142.99 & 9.70 & VLEASIAENKKACLK & K141 & & $\mathrm{X}$ \\
\hline & 17.93 & 5.68 & SFHEPPKLLPSYSTHELCER & K1059 & & $x$ \\
\hline SUMO2 & 93.67 & 8.49 & GSHMADEKPK & K5 & & $\mathrm{X}$ \\
\hline
\end{tabular}

\subsubsection{Identification of SUMO-Conjugated Sites in vivo}

Although the identification of SUMO conjugation sites in endogenous proteins from yeast has been performed before [177], unbiased identification of SUMO acceptor sites in higher eukaryotes has remained a technical challenge. This can partly be accounted for by the high mass of SUMO after hydrolysis with trypsin in higher eukaryotes combined with the low abundance of post-translational modifications per se as compared with the amount of non-modified protein. Additionally, chemical enrichment for modifications with SUMO prior to MS has not been described, as is the case for instance with phosphorylation [75, 103, 194]. To examine the power of our strategy for identification of SUMO conjugation sites we purified endogenous SUMO1 conjugates from HeLa cells shown in Figure 5.8. Although the overall protein composition in the immunoprecipitation of SUMO1 conjugates seem indistinguishable from the control IP in Coomassie blue staining, the western blot clearly demonstrates enrichment of SUMO1 conjugates in the IP (Figure 5.8A). The gel was cut into slices and the proteins specifically present in the SUMO1 immunoprecipitation were identified by LC-MS/MS. One of the most prominent SUMO1 conjugates was found at 90kDa and represents RanGAP1 
conjugated with SUMO1 [187]. By applying our ChopNSpice approach, we were able to identify lysine 524 in endogenous RanGAP1 with endogenous SUMO1 (Figure 5.8B).

(A)

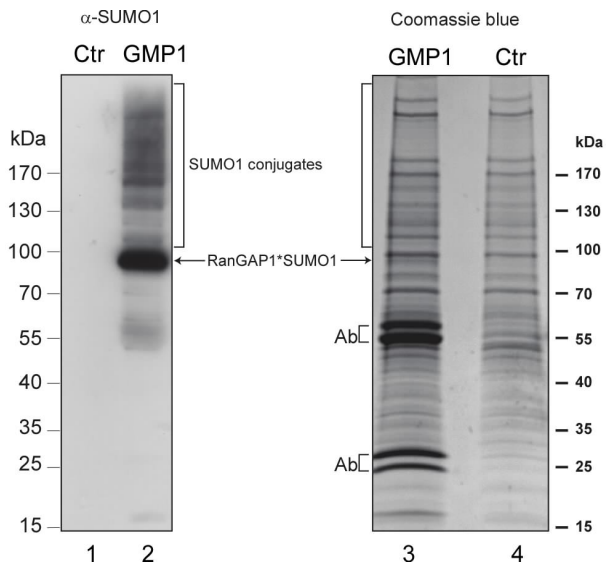

(B)

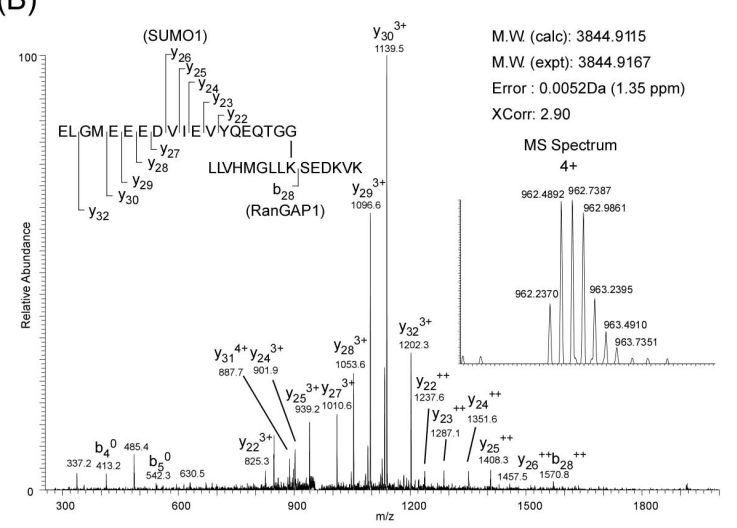

Figure 5.8. Identification of SUMO acceptor sites in endogenous proteins. (A) SUMO1 conjugated proteins were isolated from HeLa cells using SUMO1 antibodies coupled to protein $G$ agarose or control protein $G$ agarose. Immunoprecipitates were extensively washed and eluted with Sample buffer. Five percent of the sample was loaded to detect SUMO1 conjugated species by Western blot (left panel), the rest of the sample was used for to identify SUMO acceptor sites by MS (right panel). RanGAP1 conjugated with SUMO1 is indicated by the arrows. (B) MS/MS CID spectrum of a tryptic peptide ( $\mathrm{m} / \mathrm{z}=962.2370$ ) derived from RanGAP1 encompassing positions 516-530 with fragment ions recorded in the LTQ-Orbitrap MS. MS/MS in combination with database searches of the modified RanGAP1 sequence (by ChopNSpice) confirmed the known Lys-524 as actual SUMO site. $\mathrm{Y}$ - and b-type ions are shown in the spectrum and at their respective positions in the conjugated peptide. XCorr is the score in the database search using Sequest.

Importantly, in a subsequent experiment, we could additionally identify SUMO acceptor lysine residues in SUMO 1, SUMO 2/3, Ubc9, RanBP2, and others. Although several of these proteins were known as SUMO targets, the SUMO acceptor sites within RanBP2 have not been described before. Interestingly, in the SUMO1 immuno-precipitate we also observe SUMO2 conjugated to SUMO2 on Lysine 11. In total, 10 SUMOylated proteins corresponding to 17 distinct sites were identified. 15 SUMOylated sites were identified in this study appeared to be novel, which may provide a valuable resource to the biological research community. shown in Table 5.3. Thus, our MS approach proved to be highly reliable, and it easily and specifically identified SUMO acceptor sites both in vitro and in vivo. Thereby, our method increases the sensitivity of the identification of SUMO conjugation sites in mammalian cells. 
Table 5.3. In vivo SUMOylated proteins derived after immunoprecipitation from HeLa cells using anti-SUMO 1 antibody. The sequence of the SUMOylated peptide and the positions of SUMO-acceptor sites as determined by MS and MS/MS using "ChopNSpice" in combination with MASCOT and Sequest as search engines are listed.

\begin{tabular}{|c|c|c|c|c|c|}
\hline Modification & Conjugated Protein Name & Sequence & Site & $\begin{array}{l}\text { Mascot } \\
\text { Score }\end{array}$ & $\begin{array}{l}\text { Sequest } \\
\text { XC }\end{array}$ \\
\hline \multirow[t]{17}{*}{ SUMO1 } & Ran GTPase-activating protein 1 & ${ }^{516}$ LLVHMGLLKSEDKVK ${ }^{530}$ & K524 & 70,29 & 6,10 \\
\hline & Small ubiquitin-related modifier 1 & ${ }^{17}$ KEGEYIK ${ }^{23}$ & K17 & 92,87 & 5,60 \\
\hline & Small ubiquitin-related modifier 2 & ${ }^{8}$ EGVKTENNDHINLK ${ }^{21}$ & K11 & 79,50 & 5,57 \\
\hline & SUMO-conjugating enzyme UBC9 & ${ }^{142}$ VEYEKR ${ }^{147}$ & K146 & 59,84 & 5,18 \\
\hline & E3 SUMO-protein ligase RanBP2 & ${ }^{100}$ IAELLCKNDVTDGRAKYWLER $^{120}$ & - & 14,31 & 4,34 \\
\hline & & ${ }^{1408}$ FALVTPKK ${ }^{1415}$ & K1414 & 36,53 & 4,22 \\
\hline & & ${ }^{1715}$ SGFEGMFTKK ${ }^{1724}$ & K1723 & 106,23 & 5,46 \\
\hline & & ${ }^{2255} \mathrm{KNLFR}^{2259}$ & K2255 & 35,55 & 2,86 \\
\hline & & ${ }^{2424}$ FKLQDVADSFKK ${ }^{2434}$ & K2433 & 94,41 & 5,12 \\
\hline & & ${ }^{2507}$ AVVSPPKFVFGSESVK ${ }^{2522}$ & K2513 & 29,65 & 2,59 \\
\hline & & ${ }^{2581}$ NSDIEQSSDSKVK ${ }^{2594}$ & K2592 & 97,71 & 6,57 \\
\hline & & ${ }^{2617} \mathrm{AKEK}^{2620}$ & K2618 & 44,60 & 6,22 \\
\hline & $\begin{array}{l}\text { RANBP2-like and GRIP } \\
\text { domain-containing protein } 4\end{array}$ & ${ }^{691}$ KAEDIANDALSPEEQEECK ${ }^{709}$ & K691 & 7,29 & - \\
\hline & $\begin{array}{l}\text { Chromodomain-helicase-DNA- } \\
\text { binding protein } 8\end{array}$ & ${ }^{581}$ YTEDLDIKITDDEEEEEVDVTGPIK ${ }^{609}$ & K592 & - & 4,02 \\
\hline & Cytoplasmic dynein 1 heavy chain 1 & ${ }^{3207}$ KIKETVDQVEELR ${ }^{3219}$ & K3207 & 20,56 & 4,21 \\
\hline & $\begin{array}{l}\text { Very long-chain specific acyl-CoA } \\
\text { dehydrogenase }\end{array}$ & ${ }^{633} \mathrm{NFKSISK}^{639}$ & K635 & 46,13 & - \\
\hline & $\begin{array}{l}\text { Bifunctional aminoacyl-tRNA } \\
\text { synthetase }\end{array}$ & ${ }^{314}$ NPIEKNLQMWEEMK ${ }^{327}$ & K318 & 51,70 & - \\
\hline SUMO2 & Small ubiquitin-related modifier 2 & ${ }^{8}$ EGVKTENNDHINLK ${ }^{21}$ & $\mathrm{~K} 11$ & 37,99 & 7,31 \\
\hline
\end{tabular}

\subsection{Conclusion}

In this study, we present a freely available computational approach to identify SUMO acceptor sites in endogenous proteins by mass spectrometry that cannot easily be explored by using common search engines such as SEQUEST and/or MASCOT in a rapid and sensitive manner. The software "ChopNspice" for the identification of SUMO acceptor sites is unique in its ability to allow the user (I) to combine two protein sequences in a linear manner; (II) to generate any modified linear protein sequence that contains any modifications at the $\mathrm{N}$-terminus of the novel fused sequence; (III) to introduce defined extra masses in either of the two protein sequences so that also peptide-peptide crosslink (using a crosslinking reagent) after enzymatic digestion of crosslinked proteins can be explored and identified, and (IV) to generate a $\mathrm{m} / \mathrm{z}$ list of all linearly fused peptides. The latter is particularly useful when users do not have access to e.g. an Orbitrap MS or FT-IRC MS but instead would like to use a simple peptide mass fingerprint analysis by MALDI-MS of putatively SUMOylated proteins. In addition, the list serves as inclusion list in LC-MS/MS analysis such that predicted modified (e.g. SUMOylated) peptides 
are chosen for fragmentation within the mass spectrometer. By using ChopNspice, a multiple SUMOylated FASTA sequence of a proteins is generated in silico. The new FASTA sequence is implemented in the MS-based database search engines to determine the SUMOylated sites. The high-mass data dependent acquisition is highly suitable for the accurate detection and sequencing of larger peptides and additionally facilitates detection of lower abundant SUMO conjugations. In conclusion, we demonstrate our approach is of value in MS-based analysis and subsequent database search for the identification of SUMO-conjugated sites in a more efficient time scale. We believe that this approach has the potential to be widely used in the foreseeable future. 


\section{References}

[1] Venter, J. C., Adams, M. D., Myers, E. W., Li, P. W., et al., The sequence of the human genome. Science 2001, 291, 1304-1351.

[2] Lander, E. S., Linton, L. M., Birren, B., Nusbaum, C., et al., Initial sequencing and analysis of the human genome. Nature 2001, 409, 860-921.

[3] Wasinger, V. C., Cordwell, S. J., Cerpa-Poljak, A., Yan, J. X., et al., Progress with gene-product mapping of the Mollicutes: Mycoplasma genitalium. Electrophoresis 1995, 16, 1090-1094.

[4] Wilkins, M. R., Pasquali, C., Appel, R. D., Ou, K., et al., From proteins to proteomes: large scale protein identification by two-dimensional electrophoresis and amino acid analysis. Nature Biotechnology 1996, 14, 61-65.

[5] James, P., Protein identification in the post-genome era: the rapid rise of proteomics. $Q$ Rev Biophys 1997, 30, 279-331.

[6] van Weeren, P. C., de Bruyn, K. M., de Vries-Smits, A. M., van Lint, J., Burgering, B. M., Essential role for protein kinase $B(P K B)$ in insulin-induced glycogen synthase kinase 3 inactivation. Characterization of dominant-negative mutant of PKB. J Biol Chem 1998, 273, 13150-13156.

[7] Thrower, J. S., Hoffman, L., Rechsteiner, M., Pickart, C. M., Recognition of the polyubiquitin proteolytic signal. EMBO J 2000, 19, 94-102.

[8] Hay, R. T., SUMO: a history of modification. Mol Cell 2005, 18, 1-12.

[9] Shapiro, A. L., Vinuela, E., Maizel, J. V., Jr., Molecular weight estimation of polypeptide chains by electrophoresis in SDS-polyacrylamide gels. Biochem Biophys Res Commun 1967 , 28, 815-820.

[10] Weber, K., Osborn, M., The reliability of molecular weight determinations by dodecyl sulfate-polyacrylamide gel electrophoresis. J Biol Chem 1969, 244, 4406-4412.

[11] O'Farrell, P. H., High resolution two-dimensional electrophoresis of proteins. J Biol Chem 1975, 250, 4007-4021.

[12] Gorg, A., Weiss, W., Dunn, M. J., Current two-dimensional electrophoresis technology for proteomics. Proteomics 2004, 4, 3665-3685.

[13] Rabilloud, T., Two-dimensional gel electrophoresis in proteomics: old, old fashioned, but it still climbs up the mountains. Proteomics 2002, 2, 3-10.

[14] Pappin, D. J., Hojrup, P., Bleasby, A. J., Rapid identification of proteins by peptide-mass fingerprinting. Curr Biol 1993, 3, 327-332.

[15] Henzel, W. J., Billeci, T. M., Stults, J. T., Wong, S. C., et al., Identifying proteins from two-dimensional gels by molecular mass searching of peptide fragments in protein sequence databases. Proc Natl Acad Sci U S A 1993, 90, 5011-5015.

[16] Mann, M., Hojrup, P., Roepstorff, P., Use of Mass-Spectrometric Molecular-Weight Information to Identify Proteins in Sequence Databases. Biol Mass Spectrom 1993, 22, 
338-345.

[17] James, P., Quadroni, M., Carafoli, E., Gonnet, G., Protein identification by mass profile fingerprinting. Biochem Biophys Res Commun 1993, 195, 58-64.

[18] Yates, J. R., 3rd, Speicher, S., Griffin, P. R., Hunkapiller, T., Peptide mass maps: a highly informative approach to protein identification. Anal Biochem 1993, 214, 397-408.

[19] Link, A. J., Eng, J., Schieltz, D. M., Carmack, E., et al., Direct analysis of protein complexes using mass spectrometry. Nat Biotechnol 1999, 17, 676-682.

[20] Washburn, M. P., Wolters, D., Yates, J. R., 3rd, Large-scale analysis of the yeast proteome by multidimensional protein identification technology. Nat Biotechnol 2001, 19, 242-247.

[21] Kim, S. C., Sprung, R., Chen, Y., Xu, Y., et al., Substrate and functional diversity of lysine acetylation revealed by a proteomics survey. Mol Cell 2006, 23, 607-618.

[22] Ong, S. E., Mittler, G., Mann, M., Identifying and quantifying in vivo methylation sites by heavy methyl SILAC. Nat Methods 2004, 1, 119-126.

[23] Ross, A. H., Baltimore, D., Eisen, H. N., Phosphotyrosine-containing proteins isolated by affinity chromatography with antibodies to a synthetic hapten. Nature 1981, 294, 654-656.

[24] Cummings, R. D., Kornfeld, S., Fractionation of asparagine-linked oligosaccharides by serial lectin-Agarose affinity chromatography. A rapid, sensitive, and specific technique. J Biol Chem 1982, 257, 11235-11240.

[25] Machida, K., Mayer, B. J., Nollau, P., Profiling the global tyrosine phosphorylation state. Mol Cell Proteomics 2003, 2, 215-233.

[26] Oda, Y., Nagasu, T., Chait, B. T., Enrichment analysis of phosphorylated proteins as a tool for probing the phosphoproteome. Nat Biotechnol 2001, 19, 379-382.

[27] Wells, L., Vosseller, K., Cole, R. N., Cronshaw, J. M., et al., Mapping sites of O-GlcNAc modification using affinity tags for serine and threonine post-translational modifications. $\mathrm{Mol}$ Cell Proteomics 2002, 1, 791-804.

[28] Fenn, J. B., Mann, M., Meng, C. K., Wong, S. F., Whitehouse, C. M., Electrospray ionization for mass spectrometry of large biomolecules. Science 1989, 246, 64-71.

[29] Tanaka, K., The origin of macromolecule ionization by laser irradiation (Nobel lecture). Angew Chem Int Ed Engl 2003, 42, 3860-3870.

[30] Karas, M., Bachmann, D., Hillenkamp, F., Influence of the Wavelength in High-Irradiance Ultraviolet-Laser Desorption Mass-Spectrometry of Organic-Molecules. Analytical Chemistry 1985, 57, 2935-2939.

[31] Karas, M., Hillenkamp, F., Laser desorption ionization of proteins with molecular masses exceeding 10,000 daltons. Anal Chem 1988, 60, 2299-2301.

[32] Taylor, G., Disintegration of Water Drops in Electric Field. Proc R Soc Lon Ser-A 1964, 280 , 383-+.

[33] Wilm, M. S., Mann, M., Electrospray and Taylor-Cone Theory, Doles Beam of 
Macromolecules at Last. Int J Mass Spectrom 1994, 136, 167-180.

[34] Hillenkamp, F., Karas, M., Beavis, R. C., Chait, B. T., Matrix-assisted laser desorption/ionization mass spectrometry of biopolymers. Anal Chem 1991, 63, 1193A-1203A.

[35] Aebersold, R., Mann, M., Mass spectrometry-based proteomics. Nature 2003, 422, 198-207.

[36] Leary, J. J., Schmidt, R. L., Quadrupole mass spectrometers: An intuitive leak at the math. J Chem Educ 1996, 73, 1142-1145.

[37] Steel, C., Henchman, M., Understanding the quadrupole mass filter through computer simulation. J Chem Educ 1998, 75, 1049-1054.

[38] Domon, B., Aebersold, R., Mass spectrometry and protein analysis. Science 2006, 312, 212-217.

[39] Vanberkel, G. J., Glish, G. L., Mcluckey, S. A., Electrospray lonization Combined with Ion Trap Mass-Spectrometry. Analytical Chemistry 1990, 62, 1284-1295.

[40] Jonscher, K. R., Yates, J. R., 3rd, The quadrupole ion trap mass spectrometer--a small solution to a big challenge. Anal Biochem 1997, 244, 1-15.

[41] Weickhardt, C., Moritz, F., Grotemeyer, J., Time-of-flight mass spectrometry: State-of-the-art in chemical analysis and molecular science. Mass Spectrometry Reviews 1996, $15,139-162$.

[42] Marshall, A. G., Hendrickson, C. L., Jackson, G. S., Fourier transform ion cyclotron resonance mass spectrometry: a primer. Mass Spectrom Rev 1998, 17, 1-35.

[43] Makarov, A., Electrostatic axially harmonic orbital trapping: a high-performance technique of mass analysis. Anal Chem 2000, 72, 1156-1162.

[44] Hu, Q., Noll, R. J., Li, H., Makarov, A., et al., The Orbitrap: a new mass spectrometer. $J$ Mass Spectrom 2005, 40, 430-443.

[45] Chernushevich, I. V., Loboda, A. V., Thomson, B. A., An introduction to quadrupole-time-of-flight mass spectrometry. J Mass Spectrom 2001, 36, 849-865.

[46] Medzihradszky, K. F., Campbell, J. M., Baldwin, M. A., Falick, A. M., et al., The characteristics of peptide collision-induced dissociation using a high-performance MALDI-TOF/TOF tandem mass spectrometer. Analytical Chemistry 2000, 72, 552-558.

[47] Roepstorff, P., Fohlman, J., Proposal for a Common Nomenclature for Sequence Ions in Mass-Spectra of Peptides. Biomed Mass Spectrom 1984, 11, 601-601.

[48] Johnson, R. S., Martin, S. A., Biemann, K., Stults, J. T., Watson, J. T., Novel Fragmentation Process of Peptides by Collision-Induced Decomposition in a Tandem Mass-Spectrometer - Differentiation of Leucine and Isoleucine. Analytical Chemistry 1987, 59, 2621-2625.

[49] Eng, J. K., Mccormack, A. L., Yates, J. R., An Approach to Correlate Tandem Mass-Spectral Data of Peptides with Amino-Acid-Sequences in a Protein Database. J Am Soc Mass Spectr 1994, 5, 976-989. 


\section{References}

[50] Perkins, D. N., Pappin, D. J. C., Creasy, D. M., Cottrell, J. S., Probability-based protein identification by searching sequence databases using mass spectrometry data. Electrophoresis 1999, 20, 3551-3567.

[51] Will, C. L., Luhrmann, R., In: Gesteland, R. F., Cech, J. F., and Atkins, J. F., eds. The RNA World, 3rd Ed., Spliceosome Structure and Function, Cold Spring Harbor Laboratory Press, Cold Spring Harbor, N.Y. 2006, 369-400.

[52] Mermoud, J. E., Cohen, P. T., Lamond, A. I., Regulation of mammalian spliceosome assembly by a protein phosphorylation mechanism. EMBO J 1994, 13, 5679-5688.

[53] Misteli, T., RNA splicing: What has phosphorylation got to do with it? Curr Biol 1999, 9 , R198-+.

[54] Bollen, M., Beullens, M., Signaling by protein phosphatases in the nucleus. Trends Cell Biol 2002, 12, 138-145.

[55] Soret, J., Tazi, J., Phosphorylation-dependent control of the pre-mRNA splicing machinery. Prog Mol Subcell Biol 2003, 31, 89-126.

[56] Hunter, T., Signaling--2000 and beyond. Cell 2000, 100, 113-127.

[57] Mann, M., Ong, S. E., Gronborg, M., Steen, H., et al., Analysis of protein phosphorylation using mass spectrometry: deciphering the phosphoproteome. Trends Biotechnol 2002, 20 , 261-268.

[58] Mann, M., Jensen, O. N., Proteomic analysis of post-translational modifications. Nat Biotechnol 2003, 21, 255-261.

[59] Kalume, D. E., Molina, H., Pandey, A., Tackling the phosphoproteome: tools and strategies. Curr Opin Chem Biol 2003, 7, 64-69.

[60] Peters, E. C., Brock, A., Ficarro, S. B., Exploring the phosphoproteome with mass spectrometry. Mini Rev Med Chem 2004, 4, 313-324.

[61] Conrads, T. P., Issaq, H. J., Veenstra, T. D., New tools for quantitative phosphoproteome analysis. Biochem Biophys Res Commun 2002, 290, 885-890.

[62] Motoyama, A., Xu, T., Ruse, C. I., Wohlschlegel, J. A., Yates, J. R., 3rd, Anion and cation mixed-bed ion exchange for enhanced multidimensional separations of peptides and phosphopeptides. Anal Chem 2007, 79, 3623-3634.

[63] Lim, K. B., Kassel, D. B., Phosphopeptides enrichment using on-line two-dimensional strong cation exchange followed by reversed-phase liquid chromatography/mass spectrometry. Analytical Biochemistry 2006, 354, 213-219.

[64] Dai, J., Jin, W. H., Sheng, Q. H., Shieh, C. H., et al., Protein phosphorylation and expression profiling by Yin-yang multidimensional liquid chromatography (Yin-yang MDLC) mass spectrometry. J Proteome Res 2007, 6, 250-262.

[65] Beausoleil, S. A., Jedrychowski, M., Schwartz, D., Elias, J. E., et al., Large-scale characterization of HeLa cell nuclear phosphoproteins. Proc Natl Acad Sci U S A 2004, 101, 12130-12135. 


\section{References}

[66] Ballif, B. A., Villen, J., Beausoleil, S. A., Schwartz, D., Gygi, S. P., Phosphoproteomic analysis of the developing mouse brain. Mol Cell Proteomics 2004, 3, 1093-1101.

[67] Machida, M., Kosako, H., Shirakabe, K., Kobayashi, M., et al., Purification of phosphoproteins by immobilized metal affinity chromatography and its application to phosphoproteome analysis. FEBS J 2007, 274, 1576-1587.

[68] Dubrovska, A., Souchelnytskyi, S., Efficient enrichment of intact phosphorylated proteins by modified immobilized metal-affinity chromatography. Proteomics 2005, 5, 4678-4683.

[69] Zhang, X., Ye, J., Jensen, O. N., Roepstorff, P., Highly Efficient Phosphopeptide Enrichment by Calcium Phosphate Precipitation Combined with Subsequent IMAC Enrichment. Mol Cell Proteomics 2007, 6, 2032-2042.

[70] Andersson, L., Porath, J., Isolation of phosphoproteins by immobilized metal $(\mathrm{Fe} 3+$ ) affinity chromatography. Anal Biochem 1986, 154, 250-254.

[71] Muszynska, G., Dobrowolska, G., Medin, A., Ekman, P., Porath, J. O., Model studies on iron(III) ion affinity chromatography. II. Interaction of immobilized iron(III) ions with phosphorylated amino acids, peptides and proteins. J Chromatogr 1992, 604, 19-28.

[72] Gruhler, A., Olsen, J. V., Mohammed, S., Mortensen, P., et al., Quantitative phosphoproteomics applied to the yeast pheromone signaling pathway. Mol Cell Proteomics 2005, 4, 310-327.

[73] Pandey, A., Andersen, J. S., Mann, M., Use of mass spectrometry to study signaling pathways. Sci STKE 2000, 2000, pl1.

[74] Corthals, G. L., Aebersold, R., Goodlett, D. R., Identification of phosphorylation sites using microimmobilized metal affinity chromatography. Methods Enzymol 2005, 405, 66-81.

[75] Larsen, M. R., Thingholm, T. E., Jensen, O. N., Roepstorff, P., Jorgensen, T. J., Highly selective enrichment of phosphorylated peptides from peptide mixtures using titanium dioxide microcolumns. Mol Cell Proteomics 2005, 4, 873-886.

[76] Sano, A., Nakamura, H., Titania as a chemo-affinity support for the column-switching HPLC analysis of phosphopeptides: Application to the characterization of phosphorylation sites in proteins by combination with protease digestion and electrospray ionization mass Spectrometry. Anal Sci 2004, 20, 861-864.

[77] Sano, A., Nakamura, H., Chemo-affinity of titania for the column-switching HPLC analysis of phosphopeptides. Anal Sci 2004, 20, 565-566.

[78] Kuroda, I., Shintani, Y., Motokawa, M., Abe, S., Furuno, M., Phosphopeptide-selective column-switching RP-HPLC with a titania precolumn. Anal Sci 2004, 20, 1313-1319.

[79] Pinkse, M. W., Uitto, P. M., Hilhorst, M. J., Ooms, B., Heck, A. J., Selective isolation at the femtomole level of phosphopeptides from proteolytic digests using 2D-NanoLC-ESI-MS/MS and titanium oxide precolumns. Anal Chem 2004, 76, 3935-3943.

[80] Li, Y., Liu, Y., Tang, J., Lin, H., et al., Fe3O4@Al2O3 magnetic core-shell microspheres for rapid and highly specific capture of phosphopeptides with mass spectrometry analysis. $J$ 
Chromatogr A 2007, 1172, 57-71.

[81] Li, Y., Lin, H. Q., Deng, C. H., Yang, P. Y., Zhang, X. M., Highly selective and rapid enrichment of phosphorylated peptides using gallium oxide-coated magnetic microspheres for MALDI-TOF-MS and nano-LC-ESI-MS/MS/MS analysis. Proteomics 2008, 8, 238-249.

[82] Zhou, H., Tian, R., Ye, M., Xu, S., et al., Highly specific enrichment of phosphopeptides by zirconium dioxide nanoparticles for phosphoproteome analysis. Electrophoresis 2007, 28, 2201-2215.

[83] Cuccurullo, M., Schlosser, G., Cacace, G., Malorni, L., Pocsfalvi, G., Identification of phosphoproteins and determination of phosphorylation sites by zirconium dioxide enrichment and SELDI-MS/MS. J Mass Spectrom 2007, 42, 1069-1078.

[84] Hsiao, H. H., Hsieh, H. Y., Chou, C. C., Lin, S. Y., et al., Concerted experimental approach for sequential mapping of peptides and phosphopeptides using C18-functionalized magnetic nanoparticles. J Proteome Res 2007, 6, 1313-1324.

[85] Li, Y., Leng, T. H., Lin, H. Q., Deng, C. H., et al., Preparation of Fe3O4@ZrO2 core-shell microspheres as affinity probes for selective enrichment and direct determination of phosphopeptides using matrix-assisted laser desorption ionization mass spectrometry. Journal of Proteome Research 2007, 6, 4498-4510.

[86] Chen, C. T., Chen, W. Y., Tsai, P. J., Chien, K. Y., et al., Rapid enrichment of phosphopeptides and phosphoproteins from complex samples using magnetic particles coated with alumina as the concentrating probes for MALDI MS analysis. Journal of Proteome Research 2007, 6, 316-325.

[87] Kweon, H. K., Hakansson, K., Selective zirconium dioxide-based enrichment of phosphorylated peptides for mass spectrometric analysis. Anal Chem 2006, 78, 1743-1749.

[88] Bodenmiller, B., Mueller, L. N., Mueller, M., Domon, B., Aebersold, R., Reproducible isolation of distinct, overlapping segments of the phosphoproteome. Nat Methods 2007, 4, 231-237.

[89] Zheng, H., Hu, P., Quinn, D. F., Wang, Y. K., Phosphotyrosine proteomic study of interferon alpha signaling pathway using a combination of immunoprecipitation and immobilized metal affinity chromatography. Mol Cell Proteomics 2005, 4, 721-730.

[90] Stupak, J., Liu, H., Wang, Z., Brix, B. J., et al., Nanoliter sample handling combined with microspot MALDI-MS for detection of gel-separated phosphoproteins. J Proteome Res 2005, 4, 515-522.

[91] Kim, J. E., Tannenbaum, S. R., White, F. M., Global phosphoproteome of HT-29 human colon adenocarcinoma cells. J Proteome Res 2005, 4, 1339-1346.

[92] Ficarro, S. B., McCleland, M. L., Stukenberg, P. T., Burke, D. J., et al., Phosphoproteome analysis by mass spectrometry and its application to Saccharomyces cerevisiae. Nat Biotechnol 2002, 20, 301-305.

[93] Luhrmann, R., Appel, B., Bringmann, P., Rinke, J., et al., Isolation and characterization of 
rabbit anti-m3 2,2,7G antibodies. Nucleic Acids Res 1982, 10, 7103-7113.

[94] Bringmann, P., Luhrmann, R., Purification of the individual snRNPs U1, U2, U5 and U4/U6 from HeLa cells and characterization of their protein constituents. EMBO J 1986, 5, 3509-3516.

[95] Behrens, S. E., Luhrmann, R., Immunoaffinity purification of a [U4/U6.U5] tri-snRNP from human cells. Genes Dev 1991, 5, 1439-1452.

[96] Bessonov, S., Anokhina, M., Will, C. L., Urlaub, H., Luhrmann, R., Isolation of an active step I spliceosome and composition of its RNP core. Nature 2008, 452, 846-850.

[97] Deckert, J., Hartmuth, K., Boehringer, D., Behzadnia, N., et al., Protein composition and electron microscopy structure of affinity-purified human spliceosomal B complexes isolated under physiological conditions. Mol Cell Biol 2006, 26, 5528-5543.

[98] Zahler, A. M., Purification of SR protein splicing factors. Methods Mol Biol 1999, 118, 419-432.

[99] Lee, C. L., Hsiao, H. H., Lin, C. W., Wu, S. P., et al., Strategic shotgun proteomics approach for efficient construction of an expression map of targeted protein families in hepatoma cell lines. Proteomics 2003, 3, 2472-2486.

[100] Schwartz, D., Gygi, S. P., An iterative statistical approach to the identification of protein phosphorylation motifs from large-scale data sets. Nat Biotechnol 2005, 23, 1391-1398.

[101] Schneider, T. D., Stephens, R. M., Sequence logos: a new way to display consensus sequences. Nucleic Acids Res 1990, 18, 6097-6100.

[102] Mazanek, M., Mituloviae, G., Herzog, F., Stingl, C., et al., Titanium dioxide as a chemo-affinity solid phase in offline phosphopeptide chromatography prior to HPLC-MS/MS analysis. Nat Protoc 2007, 2, 1059-1069.

[103] Thingholm, T. E., Jorgensen, T. J., Jensen, O. N., Larsen, M. R., Highly selective enrichment of phosphorylated peptides using titanium dioxide. Nat Protoc 2006, 1, 1929-1935. [104] Stark, G. R., Stein, W. H., Moore, S., Reactions of Cyanate Present in Aqueous Urea with Amino Acids and Proteins. J Biol Chem 1960, 235, 3177-3181.

[105] Stark, G. R., Reactions of Cyanate with Functional Groups of Proteins .3. Reactions with Amino and Carboxyl Groups. Biochemistry 1965, 4, 1030-\&.

[106] Stark, G. R., Modification of proteins with cyanate. Methods Enzymol 1967, 11, 590-594. [107] Yu, Y. Q., Gilar, M., Lee, P. J., Bouvier, E. S., Gebler, J. C., Enzyme-friendly, mass spectrometry-compatible surfactant for in-solution enzymatic digestion of proteins. Anal Chem 2003, 75, 6023-6028.

[108] Ishihama, Y., Rappsilber, J., Mann, M., Modular stop and go extraction tips with stacked disks for parallel and multidimensional Peptide fractionation in proteomics. J Proteome Res 2006, 5, 988-994.

[109] Rappsilber, J., Ishihama, Y., Mann, M., Stop and go extraction tips for matrix-assisted laser desorption/ionization, nanoelectrospray, and LC/MS sample pretreatment in proteomics. 
Anal Chem 2003, 75, 663-670.

[110] Yu, S. Y., Wu, S. W., Hsiao, H. H., Khoo, K. H., Enabling techniques and strategic workflow for sulfoglycomics based on mass spectrometry mapping and sequencing of permethylated sulfated glycans. Glycobiology 2009, 19, 1136-1149.

[111] Jensen, S. S., Larsen, M. R., Evaluation of the impact of some experimental procedures on different phosphopeptide enrichment techniques. Rapid Commun Mass Sp 2007, 21, 3635-3645.

[112] Stensballe, A., Jensen, O. N., Phosphoric acid enhances the performance of Fe(III) affinity chromatography and matrix-assisted laser desorption/ionization tandem mass spectrometry for recovery, detection and sequencing of phosphopeptides. Rapid Commun Mass Spectrom 2004, 18, 1721-1730.

[113] Chen, H. S., Rejtar, T., Andreev, V., Moskovets, E., Karger, B. L., Enhanced characterization of complex proteomic samples using LC-MALDI MS/MS: exclusion of redundant peptides from MS/MS analysis in replicate runs. Anal Chem 2005, 77, 7816-7825.

[114] Gnad, F., Ren, S., Cox, J., Olsen, J. V., et al., PHOSIDA (phosphorylation site database): management, structural and evolutionary investigation, and prediction of phosphosites. Genome Biol 2007, 8, R250.

[115] Manning, B. D., Cantley, L. C., Hitting the target: emerging technologies in the search for kinase substrates. Sci STKE 2002, 2002, pe49.

[116] Manley, J. L., Tacke, R., SR proteins and splicing control. Genes Dev 1996, 10, 1569-1579.

[117] Sanford, J. R., Longman, D., Caceres, J. F., Multiple roles of the SR protein family in splicing regulation. Prog Mol Subcell Biol 2003, 31, 33-58.

[118] Xiao, S. H., Manley, J. L., Phosphorylation of the ASF/SF2 RS domain affects both protein-protein and protein-RNA interactions and is necessary for splicing. Genes Dev 1997, 11, 334-344.

[119] Pearson, R. B., Kemp, B. E., Protein kinase phosphorylation site sequences and consensus specificity motifs: tabulations. Methods Enzymol 1991, 200, 62-81.

[120] Zhou, S. Y., Blechner, S., Hoagland, N., Hoekstra, M. F., et al., Use of an Oriented Peptide Library to Determine the Optimal Substrates of Protein-Kinases. Curr Biol 1994, 4, 973-982.

[121] Schneider, M., Hsiao, H. H., Will, C. L., Giet, R., et al., Human PRP4 kinase is required for stable tri-snRNP association during spliceosomal B complex formation. Nature Structural \& Molecular Biology 2010, 17, 216-U212.

[122] Oellerich, T., Gronborg, M., Neumann, K., Hsiao, H. H., et al., SLP-65 phosphorylation dynamics reveals a functional basis for signal integration by receptor-proximal adaptor proteins. Mol Cell Proteomics 2009, 8, 1738-1750.

[123] Luo, X., Hsiao, H. H., Bubunenko, M., Weber, G., et al., Structural and functional analysis 


\section{References}

of the E. coli NusB-S10 transcription antitermination complex. Mol Cell 2008, 32, 791-802.

[124] Kuhn-Holsken, E., Lenz, C., Sander, B., Luhrmann, R., Urlaub, H., Complete MALDI-ToF MS analysis of cross-linked peptide-RNA oligonucleotides derived from nonlabeled UV-irradiated ribonucleoprotein particles. RNA 2005, 11, 1915-1930.

[125] Manning, G., Whyte, D. B., Martinez, R., Hunter, T., Sudarsanam, S., The protein kinase complement of the human genome. Science 2002, 298, 1912-1934.

[126] Gronborg, M., Kristiansen, T. Z., Stensballe, A., Andersen, J. S., et al., A mass spectrometry-based proteomic approach for identification of serine/threonine-phosphorylated proteins by enrichment with phospho-specific antibodies: identification of a novel protein, Frigg, as a protein kinase A substrate. Mol Cell Proteomics 2002, 1, 517-527.

[127] Kruger, M., Kratchmarova, I., Blagoev, B., Tseng, Y. H., et al., Dissection of the insulin signaling pathway via quantitative phosphoproteomics. Proc Natl Acad Sci U S A 2008, 105, 2451-2456.

[128] Wang, Y., Li, R., Du, D., Zhang, C., et al., Proteomic analysis reveals novel molecules involved in insulin signaling pathway. J Proteome Res 2006, 5, 846-855.

[129] Zhang, G., Neubert, T. A., Use of detergents to increase selectivity of immunoprecipitation of tyrosine phosphorylated peptides prior to identification by MALDI quadrupole-TOF MS. Proteomics 2006, 6, 571-578.

[130] Tong, J., Taylor, P., Jovceva, E., St-Germain, J. R., et al., Tandem immunoprecipitation of phosphotyrosine-mass spectrometry (TIPY-MS) indicates C19ORF19 becomes tyrosine-phosphorylated and associated with activated epidermal growth factor receptor. $J$ Proteome Res 2008, 7, 1067-1077.

[131] Xia, Q., Cheng, D., Duong, D. M., Gearing, M., et al., Phosphoproteomic analysis of human brain by calcium phosphate precipitation and mass spectrometry. $J$ Proteome Res 2008, 7, 2845-2851.

[132] Schroeder, M. J., Shabanowitz, J., Schwartz, J. C., Hunt, D. F., Coon, J. J., A neutral loss activation method for improved phosphopeptide sequence analysis by quadrupole ion trap mass spectrometry. Anal Chem 2004, 76, 3590-3598.

[133] Bateman, R. H., Carruthers, R., Hoyes, J. B., Jones, C., et al., A novel precursor ion discovery method on a hybrid quadrupole orthogonal acceleration time-of-flight (Q-TOF) mass spectrometer for studying protein phosphorylation. J Am Soc Mass Spectrom 2002, 13 , 792-803.

[134] Leimgruber, R. M., Malone, J. P., Radabaugh, M. R., LaPorte, M. L., et al., Development of improved cell lysis, solubilization and imaging approaches for proteomic analyses. Proteomics 2002, 2, 135-144.

[135] Nielsen, P. A., Olsen, J. V., Podtelejnikov, A. V., Andersen, J. R., et al., Proteomic mapping of brain plasma membrane proteins. Mol Cell Proteomics 2005, 4, 402-408.

[136] Chen, E. I., Cociorva, D., Norris, J. L., Yates, J. R., 3rd, Optimization of mass 
spectrometry-compatible surfactants for shotgun proteomics. J Proteome Res 2007, 6, 2529-2538.

[137] Tazi, J., Kornstadt, U., Rossi, F., Jeanteur, P., et al., Thiophosphorylation of U1-70K protein inhibits pre-mRNA splicing. Nature 1993, 363, 283-286.

[138] Woppmann, A., Patschinsky, T., Bringmann, P., Godt, F., Luhrmann, R., Characterisation of human and murine snRNP proteins by two-dimensional gel electrophoresis and phosphopeptide analysis of U1-specific 70K protein variants. Nucleic Acids Res 1990, 18, 4427-4438.

[139] Kohtz, J. D., Jamison, S. F., Will, C. L., Zuo, P., et al., Protein-protein interactions and 5'-splice-site recognition in mammalian mRNA precursors. Nature 1994, 368, 119-124.

[140] Raker, V. A., Plessel, G., Luhrmann, R., The snRNP core assembly pathway: identification of stable core protein heteromeric complexes and an snRNP subcore particle in vitro. EMBO J 1996, 15, 2256-2269.

[141] Varki, A., Biological roles of oligosaccharides: all of the theories are correct. Glycobiology 1993, 3, 97-130.

[142] Van den Steen, P., Rudd, P. M., Dwek, R. A., Opdenakker, G., Concepts and principles of O-linked glycosylation. Crit Rev Biochem Mol Biol 1998, 33, 151-208.

[143] Calvete, J. J., Sanz, L., Analysis of O-glycosylation. Methods Mol Biol 2008, 446, 281-292.

[144] Marshall, R. D., The nature and metabolism of the carbohydrate-peptide linkages of glycoproteins. Biochem Soc Symp 1974, 17-26.

[145] Gavel, Y., von Heijne, G., Sequence differences between glycosylated and non-glycosylated Asn-X-Thr/Ser acceptor sites: implications for protein engineering. Protein Eng 1990, 3, 433-442.

[146] Hubbard, S. C., Ivatt, R. J., Synthesis and processing of asparagine-linked oligosaccharides. Annu Rev Biochem 1981, 50, 555-583.

[147] Kornfeld, R., Kornfeld, S., Assembly of asparagine-linked oligosaccharides. Annu Rev Biochem 1985, 54, 631-664.

[148] Steen, H., Kuster, B., Fernandez, M., Pandey, A., Mann, M., Detection of tyrosine phosphorylated peptides by precursor ion scanning quadrupole TOF mass spectrometry in positive ion mode. Anal Chem 2001, 73, 1440-1448.

[149] Steen, H., Kuster, B., Mann, M., Quadrupole time-of-flight versus triple-quadrupole mass spectrometry for the determination of phosphopeptides by precursor ion scanning. $J$ Mass Spectrom 2001, 36, 782-790.

[150] Schlosser, A., Pipkorn, R., Bossemeyer, D., Lehmann, W. D., Analysis of protein phosphorylation by a combination of elastase digestion and neutral loss tandem mass spectrometry. Anal Chem 2001, 73, 170-176.

[151] Covey, T., Shushan, B., Bonner, R., Schröder, W., Hucho, F., In: H. Jornvall, J.-O. Hoog 
and A.-M. Gustavsson, Editors, Methods in protein sequence analysis, Birkhäuser Verlag, Basel ; Boston 1991, 249-256.

[152] Huddleston, M. J., Bean, M. F., Carr, S. A., Collisional fragmentation of glycopeptides by electrospray ionization LC/MS and LC/MS/MS: methods for selective detection of glycopeptides in protein digests. Anal Chem 1993, 65, 877-884.

[153] Palumbo, A. M., Reid, G. E., Evaluation of gas-phase rearrangement and competing fragmentation reactions on protein phosphorylation site assignment using collision induced dissociation-MS/MS and MS3. Anal Chem 2008, 80, 9735-9747.

[154] Palumbo, A. M., Tepe, J. J., Reid, G. E., Mechanistic insights into the multistage gas-phase fragmentation behavior of phosphoserine- and phosphothreonine-containing peptides. J Proteome Res 2008, 7, 771-779.

[155] Sullivan, B., Addona, T. A., Carr, S. A., Selective detection of glycopeptides on ion trap mass spectrometers. Anal Chem 2004, 76, 3112-3118.

[156] Rosner, M. R., Robbins, P. W., Separation of glycopeptides by high performance liquid chromatography. J Cell Biochem 1982, 18, 37-47.

[157] Geiss-Friedlander, R., Melchior, F., Concepts in sumoylation: a decade on. Nat Rev Mol Cell Biol 2007, 8, 947-956.

[158] Kerscher, O., Felberbaum, R., Hochstrasser, M., Modification of proteins by ubiquitin and ubiquitin-like proteins. Annu Rev Cell Dev Biol 2006, 22, 159-180.

[159] Meulmeester, E., Melchior, F., Cell biology: SUMO. Nature 2008, 452, 709-711.

[160] Hershko, A., Ciechanover, A., The ubiquitin system. Annu Rev Biochem 1998, 67, 425-479.

[161] Johnson, E. S., Protein modification by SUMO. Annu Rev Biochem 2004, 73, 355-382.

[162] Verger, A., Perdomo, J., Crossley, M., Modification with SUMO. A role in transcriptional regulation. EMBO Rep 2003, 4, 137-142.

[163] Bernier-Villamor, V., Sampson, D. A., Matunis, M. J., Lima, C. D., Structural basis for E2-mediated SUMO conjugation revealed by a complex between ubiquitin-conjugating enzyme Ubc9 and RanGAP1. Cell 2002, 108, 345-356.

[164] Melchior, F., SUMO--nonclassical ubiquitin. Annu Rev Cell Dev Biol 2000, 16, 591-626.

[165] Sampson, D. A., Wang, M., Matunis, M. J., The small ubiquitin-like modifier-1 (SUMO-1) consensus sequence mediates Ubc9 binding and is essential for SUMO-1 modification. J Biol Chem 2001, 276, 21664-21669.

[166] Lin, D., Tatham, M. H., Yu, B., Kim, S., et al., Identification of a substrate recognition site on Ubc9. J Biol Chem 2002, 277, 21740-21748.

[167] Melchior, F., Schergaut, M., Pichler, A., SUMO: ligases, isopeptidases and nuclear pores. Trends Biochem Sci 2003, 28, 612-618.

[168] Hay, R. T., SUMO-specific proteases: a twist in the tail. Trends Cell Biol 2007, 17, 370-376. 


\section{References}

[169] Mukhopadhyay, D., Dasso, M., Modification in reverse: the SUMO proteases. Trends Biochem Sci 2007, 32, 286-295.

[170] Mossessova, E., Lima, C. D., Ulp1-SUMO crystal structure and genetic analysis reveal conserved interactions and a regulatory element essential for cell growth in yeast. Mol Cell 2000, 5, 865-876.

[171] Steinacher, R., Schar, P., Functionality of human thymine DNA glycosylase requires SUMO-regulated changes in protein conformation. Curr Biol 2005, 15, 616-623.

[172] Baba, D., Maita, N., Jee, J. G., Uchimura, Y., et al., Crystal structure of thymine DNA glycosylase conjugated to SUMO-1. Nature 2005, 435, 979-982.

[173] Hoege, C., Pfander, B., Moldovan, G. L., Pyrowolakis, G., Jentsch, S., RAD6-dependent DNA repair is linked to modification of PCNA by ubiquitin and SUMO. Nature 2002, 419, 135-141.

[174] Pichler, A., Knipscheer, P., Oberhofer, E., van Dijk, W. J., et al., SUMO modification of the ubiquitin-conjugating enzyme E2-25K. Nat Struct Mol Biol 2005, 12, 264-269.

[175] Lin, D. Y., Huang, Y. S., Jeng, J. C., Kuo, H. Y., et al., Role of SUMO-interacting motif in Daxx SUMO modification, subnuclear localization, and repression of sumoylated transcription factors. Mol Cell 2006, 24, 341-354.

[176] Meulmeester, E., Kunze, M., Hsiao, H. H., Urlaub, H., Melchior, F., Mechanism and consequences for paralog-specific sumoylation of ubiquitin-specific protease 25. Mol Cell 2008, 30, 610-619.

[177] Denison, C., Rudner, A. D., Gerber, S. A., Bakalarski, C. E., et al., A proteomic strategy for gaining insights into protein sumoylation in yeast. Mol Cell Proteomics 2005, 4, 246-254.

[178] Vertegaal, A. C., Andersen, J. S., Ogg, S. C., Hay, R. T., et al., Distinct and overlapping sets of SUMO-1 and SUMO-2 target proteins revealed by quantitative proteomics. Mol Cell Proteomics 2006, 5, 2298-2310.

[179] Hannich, J. T., Lewis, A., Kroetz, M. B., Li, S. J., et al., Defining the SUMO-modified proteome by multiple approaches in Saccharomyces cerevisiae. J Biol Chem 2005, 280, 4102-4110.

[180] Matic, I., van Hagen, M., Schimmel, J., Macek, B., et al., In vivo identification of human small ubiquitin-like modifier polymerization sites by high accuracy mass spectrometry and an in vitro to in vivo strategy. Mol Cell Proteomics 2008, 7, 132-144.

[181] Knuesel, M., Cheung, H. T., Hamady, M., Barthel, K. K., Liu, X., A method of mapping protein sumoylation sites by mass spectrometry using a modified small ubiquitin-like modifier 1 (SUMO-1) and a computational program. Mol Cell Proteomics 2005, 4, 1626-1636.

[182] Pedrioli, P. G., Raught, B., Zhang, X. D., Rogers, R., et al., Automated identification of SUMOylation sites using mass spectrometry and SUMmOn pattern recognition software. Nat Methods 2006, 3, 533-539.

[183] Matunis, M. J., Coutavas, E., Blobel, G., A novel ubiquitin-like modification modulates the 


\section{References}

partitioning of the Ran-GTPase-activating protein RanGAP1 between the cytosol and the nuclear pore complex. J Cell Biol 1996, 135, 1457-1470.

[184] Bossis, G., Melchior, F., Regulation of SUMOylation by reversible oxidation of SUMO conjugating enzymes. Mol Cell 2006, 21, 349-357.

[185] Pichler, A., Gast, A., Seeler, J. S., Dejean, A., Melchior, F., The nucleoporin RanBP2 has SUMO1 E3 ligase activity. Cell 2002, 108, 109-120.

[186] Werner, A., Moutty, M. C., Moller, U., Melchior, F., Performing in vitro sumoylation reactions using recombinant enzymes. Methods Mol Biol 2009, 497, 187-199.

[187] Mahajan, R., Delphin, C., Guan, T., Gerace, L., Melchior, F., A small ubiquitin-related polypeptide involved in targeting RanGAP1 to nuclear pore complex protein RanBP2. Cell 1997, 88, 97-107.

[188] Maiolica, A., Cittaro, D., Borsotti, D., Sennels, L., et al., Structural analysis of multiprotein complexes by cross-linking, mass spectrometry, and database searching. Mol Cell Proteomics 2007, 6, 2200-2211.

[189] Olsen, J. V., de Godoy, L. M., Li, G., Macek, B., et al., Parts per million mass accuracy on an Orbitrap mass spectrometer via lock mass injection into a C-trap. Mol Cell Proteomics 2005, 4, 2010-2021.

[190] Olsen, J. V., Macek, B., Lange, O., Makarov, A., et al., Higher-energy C-trap dissociation for peptide modification analysis. Nat Methods 2007, 4, 709-712.

[191] Mahajan, R., Gerace, L., Melchior, F., Molecular characterization of the SUMO-1 modification of RanGAP1 and its role in nuclear envelope association. J Cell Biol 1998, 140, 259-270.

[192] Rodriguez, M. S., Desterro, J. M., Lain, S., Midgley, C. A., et al., SUMO-1 modification activates the transcriptional response of p53. EMBO J 1999, 18, 6455-6461.

[193] Sternsdorf, T., Jensen, K., Reich, B., Will, H., The nuclear dot protein sp100, characterization of domains necessary for dimerization, subcellular localization, and modification by small ubiquitin-like modifiers. J Biol Chem 1999, 274, 12555-12566.

[194] Villen, J., Gygi, S. P., The SCX/IMAC enrichment approach for global phosphorylation analysis by mass spectrometry. Nat Protoc 2008, 3, 1630-1638. 


\section{Appendixes}

Appendix 1. Phosphorylation sites identified from spliceosomal proteins by using in-house $\mathrm{TiO}_{2}$ microspin column enrichment. The underline indicates that the phosphorylation site was not identified previously.

\begin{tabular}{|c|c|c|c|}
\hline Protein & $\begin{array}{l}\text { Accession } \\
\text { Number }\end{array}$ & Phosphorylation Sites & Unassigned phosphorylation sites \\
\hline \multicolumn{4}{|l|}{ Sm proteins } \\
\hline D2 & gi|4759158 & $\underline{\mathrm{S} 9}, \underline{\mathrm{T} 12}, \underline{\mathrm{T} 40}$ & \\
\hline G & gi|4507133 & $\underline{\mathrm{T} 50}$ & \\
\hline D3 & gi|4759160 & $\underline{\mathrm{S} 44}$ & \\
\hline$E$ & gi|119589572 & $\underline{589}$ & \\
\hline D1 & gi|5902102 & $\underline{\mathrm{T} 57}$ & \\
\hline \multicolumn{4}{|l|}{ U1 snRNP } \\
\hline U1 70K & gi|36100 & 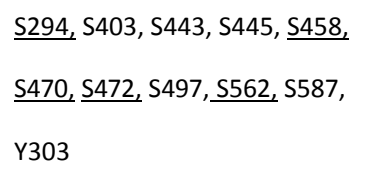 & \\
\hline U1-C & gi|4507127 & $\underline{\mathrm{S} 17}$ & \\
\hline \multicolumn{4}{|l|}{ U1 snRNP } \\
\hline RBM25 & gi|887360 & $\mathrm{S} 107, \underline{\mathrm{S} 133}, \underline{\mathrm{T} 154}, \underline{\mathrm{T} 155}$ & \\
\hline \multicolumn{4}{|l|}{ 17S U2 snRNP } \\
\hline U2A' & gi|50593002 & $\underline{\mathrm{T} 180,}, \mathrm{~S} 197$ & $\begin{array}{l}{ }^{178} \text { SKTFNPGAGLPTDKK }{ }^{192}+\mathrm{P}, \\
{ }^{236} \text { SGPTDDGEEEMEEDTVTNGS }{ }^{255}+\mathrm{P}\end{array}$ \\
\hline U2A' & gi|89063185 & & ${ }^{141}$ SGPTGDGEEEMEEDTVTNGS ${ }^{160}+\mathrm{P}$ \\
\hline SF3b145 & gi|2498883 & $\begin{array}{l}\mathrm{S} 266, \underline{\mathrm{S} 280,} \mathrm{~S} 284, \mathrm{~S} 286, \mathrm{~S} 339 \\
\mathrm{~S} 408, \mathrm{~S} 412, \mathrm{~S} 413, \mathrm{~T} 288, \mathrm{~T} 757\end{array}$ & ${ }^{336}$ GSDSPAADVEIEYVTEEPEIYEPNFIFFKR ${ }^{365}+\mathrm{P}$ \\
\hline SF3a120 & gi|5032087 & S329, S359 & \\
\hline SF3b155 & gi|15214275 & $\begin{array}{l}\underline{\mathrm{S} 73}, \underline{\mathrm{S} 129}, \underline{\mathrm{S} 190,}, \underline{\mathrm{S} 194}, \underline{\mathrm{S} 229,}, \\
\underline{\mathrm{S} 322,}, \underline{\mathrm{S} 488,}, \mathrm{~T} 142, \mathrm{~T} 207, \mathrm{~T} 211, \\
\mathrm{~T} 223, \mathrm{~T} 227, \mathrm{~T} 235, \mathrm{~T} 244, \mathrm{~T} 248, \\
\mathrm{~T} 257, \mathrm{~T} 261, \mathrm{~T} 267, \underline{\mathrm{T} 273}, \underline{\mathrm{T} 279,} \\
\mathrm{~T} 296, \underline{\mathrm{T} 303}, \mathrm{~T} 313, \mathrm{~T} 326, \mathrm{~T} 328, \\
\mathrm{~T} 426, \mathrm{~T} 434, \mathrm{~T} 436, \mathrm{~T} 442\end{array}$ & 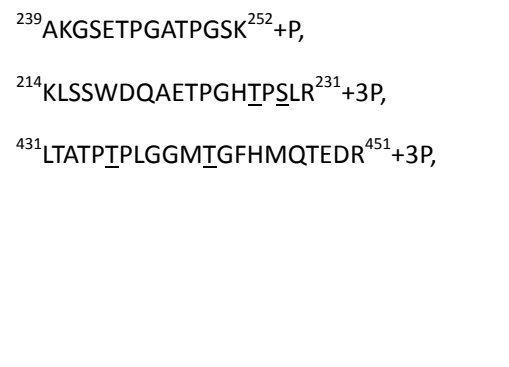 \\
\hline SF3a60 & gi|551450 & $\underline{\mathrm{S} 295}$ & \\
\hline SF3b14a/p14 & gi|7706326 & $\underline{\mathrm{T} 121}$ & \\
\hline $17 S$ U2 relatec & & & \\
\hline U2AF35 & gi|4827046 & S349, S384 & \\
\hline
\end{tabular}




\begin{tabular}{|c|c|c|c|}
\hline SF3b125 & gi|45446743 & S96, S185 & \\
\hline SPF45 & gi|14249678 & $\mathrm{S} 155, \underline{\mathrm{S} 222}$ & \\
\hline hPRP5 & gi|5410326 & S803 & \\
\hline U2AF65 & gi|6005926 & S79 & \\
\hline SR140 & gi|2224605 & $\underline{\mathrm{S} 484}$ & \\
\hline CHERP & gi|2058691 & $\underline{\mathrm{S} 781}, \underline{\mathrm{S} 783}, \underline{\mathrm{S} 785}, \underline{\mathrm{T} 787}$ & ${ }^{783}$ SRSPTPPSSAGLGSNSAPPIPDSR ${ }^{806}+3 P$ \\
\hline \multicolumn{4}{|l|}{ U5 snRNP } \\
\hline $200 K$ & gi|40217847 & $\begin{array}{l}\underline{\mathrm{S} 8}, \underline{\mathrm{S} 17}, \underline{\mathrm{S} 26}, \underline{\mathrm{S} 42}, \underline{\mathrm{S} 207, \mathrm{~S} 225} \\
\mathrm{~S} 756, \underline{\mathrm{S} 1315, \mathrm{~S} 2002}, \underline{\mathrm{S} 2133}, \\
\underline{\mathrm{S} 2135}, \underline{\mathrm{T} 393}, \underline{\mathrm{T} 1572}, \underline{\mathrm{T} 2131}\end{array}$ & $\begin{array}{l}{ }^{187} \text { EIQNMDDNIDETYGVNVQFESDEEEGDEDVYGEVR } \\
2 \mathrm{P}\end{array}$ \\
\hline $52 \mathrm{~K}$ & gi|5174409 & S49, $\underline{\text { S118, }}$ S195, $\underline{\text { T341 }}$ & $\begin{array}{l}{ }^{137} \text { QASDSEEEDSLGQTSMSAQALLEGLLELLLPR }{ }^{168}+2 \mathrm{P}, \\
{ }^{231} \text { GLGCQTLGPHNPTPPPSLDMFAEELAEEELETPTPTQR } \\
{ }^{8}+\mathrm{P},\end{array}$ \\
\hline $116 \mathrm{~K}$ & gi|41152056 & $\mathrm{S} 19, \mathrm{~T} 86, \underline{\mathrm{S} 466}, \underline{\mathrm{T} 478}, \underline{\mathrm{S} 944}$ & $\begin{array}{l}{ }^{1} \text { MDTDLYDEFGNYIGPELDSDEDDDELGR }{ }^{28}+2 \mathrm{P}, \\
{ }^{1} \mathrm{MDTDLYDEFGNYIGPELDSDEDDDELGRETK}^{31}+2 \mathrm{P}\end{array}$ \\
\hline $100 \mathrm{~K}$ & gi|67460585 & $\begin{array}{l}\mathrm{S} 14, \underline{\mathrm{S} 16}, \underline{\mathrm{S} 23}, \underline{\mathrm{S} 39}, \underline{\mathrm{S} 63}, \underline{\mathrm{S} 65}, \\
\underline{\mathrm{S} 67,} \mathrm{~S} 107, \mathrm{~S} 109, \mathrm{~T} 25\end{array}$ & ${ }^{106} \mathrm{SSLSPGRGK}^{114}+2 \mathrm{P}$ \\
\hline $102 K$ & gi|40807485 & $\begin{array}{l}\underline{\mathrm{S} 143}, \underline{\mathrm{T} 232}, \underline{\mathrm{T} 235}, \underline{\mathrm{S} 261}, \underline{\mathrm{S} 263,} \\
\underline{\mathrm{T} 266}\end{array}$ & \\
\hline $102 K$ & gi|4103604 & & $\begin{array}{l}{ }^{204} \text { QTQFGGLNTPYPGGLNTPYRGGMTPGLMTPGTGELD } \\
\mathrm{MR}^{241}+\mathrm{P}\end{array}$ \\
\hline $220 K$ & gi|73967172 & $\begin{array}{l}\underline{\mathrm{S} 1358}, \underline{\mathrm{S} 1411}, \underline{\mathrm{S} 2079,} \mathrm{~T} 2042 \\
\underline{\mathrm{T} 2044}\end{array}$ & ${ }^{2171}$ EMEPLGWIHTQPNESPQLSPQDVTTHAK ${ }^{2193}+P$ \\
\hline \multicolumn{4}{|c|}{ U4/U6 snRNP } \\
\hline $90 \mathrm{~K}$ & gi|2853287 & S618 & \\
\hline $61 \mathrm{~K}$ & gi|4914604 & $\underline{\mathrm{S} 439}, \underline{\mathrm{T} 441}, \underline{\mathrm{T} 455}$ & $\begin{array}{l}{ }^{489} \text { VKGEKSGLMST }^{499}+\mathrm{P}, \\
{ }^{445} \text { SSGTASSVAFTPLQGLEIVNPQAAEK }^{470}+3 \mathrm{P}\end{array}$ \\
\hline $60 \mathrm{~K}$ & gi|2653736 & $\underline{\mathrm{S} 482}$ & \\
\hline \multicolumn{4}{|c|}{ U4/U6.U5 Tri-snRNP } \\
\hline $110 \mathrm{~K}$ & gi|10863889 & $\begin{array}{l}\underline{\mathrm{S} 84}, \mathrm{~S} 448, \underline{\mathrm{S} 463}, \underline{\mathrm{S} 474}, \underline{\mathrm{S} 486}, \\
\mathrm{~S} 591, \mathrm{~S} 596, \mathrm{~S} 598, \underline{\mathrm{S} 623}, \mathrm{~T} 430, \\
\underline{\mathrm{T} 764}\end{array}$ & $\begin{array}{l}{ }^{587} \text { DEERSANGGSESDGEENIGWSTVNLDEEK }{ }^{615}+2 \mathrm{P}, \\
{ }^{591} \text { SANGGSESDGEENIGWSTVNLDEEKQQQDFSASSTTILD } \\
\text { EEPIVNR }^{636}+\mathrm{P}, \\
{ }^{445} \text { EPVPQPLPSDDTRVENMDISDEEEGGAPPPGSPQVLEE } \\
\text { DEAELELQK }{ }^{501}+2 \mathrm{P}\end{array}$ \\
\hline TFIP11 & gi|8393259 & $\underline{\mathrm{S} 59}, \mathrm{~S} 98, \mathrm{~S} 210, \underline{\mathrm{S} 392}$ & \\
\hline $65 \mathrm{~K}$ & gi|13926071 & $\mathrm{S} 46, \underline{\mathrm{S} 58,}, \mathrm{~S} 82, \underline{\mathrm{S} 97}$ & \\
\hline hPRP38 & gi|14042816 & $\mathrm{S} 117, \mathrm{~S} 118, \mathrm{~S} 133, \mathrm{~S} 150, \underline{\mathrm{S} 220}$ & \\
\hline $27 \mathrm{~K}$ & gi|24307919 & $\mathrm{S} 43, \mathrm{~S} 45, \underline{\mathrm{S} 61}, \underline{\mathrm{S} 63}$ & 59STSPPSPSR66+3P, ${ }^{1} M G R S R S R S P R{ }^{11}+P$ \\
\hline
\end{tabular}




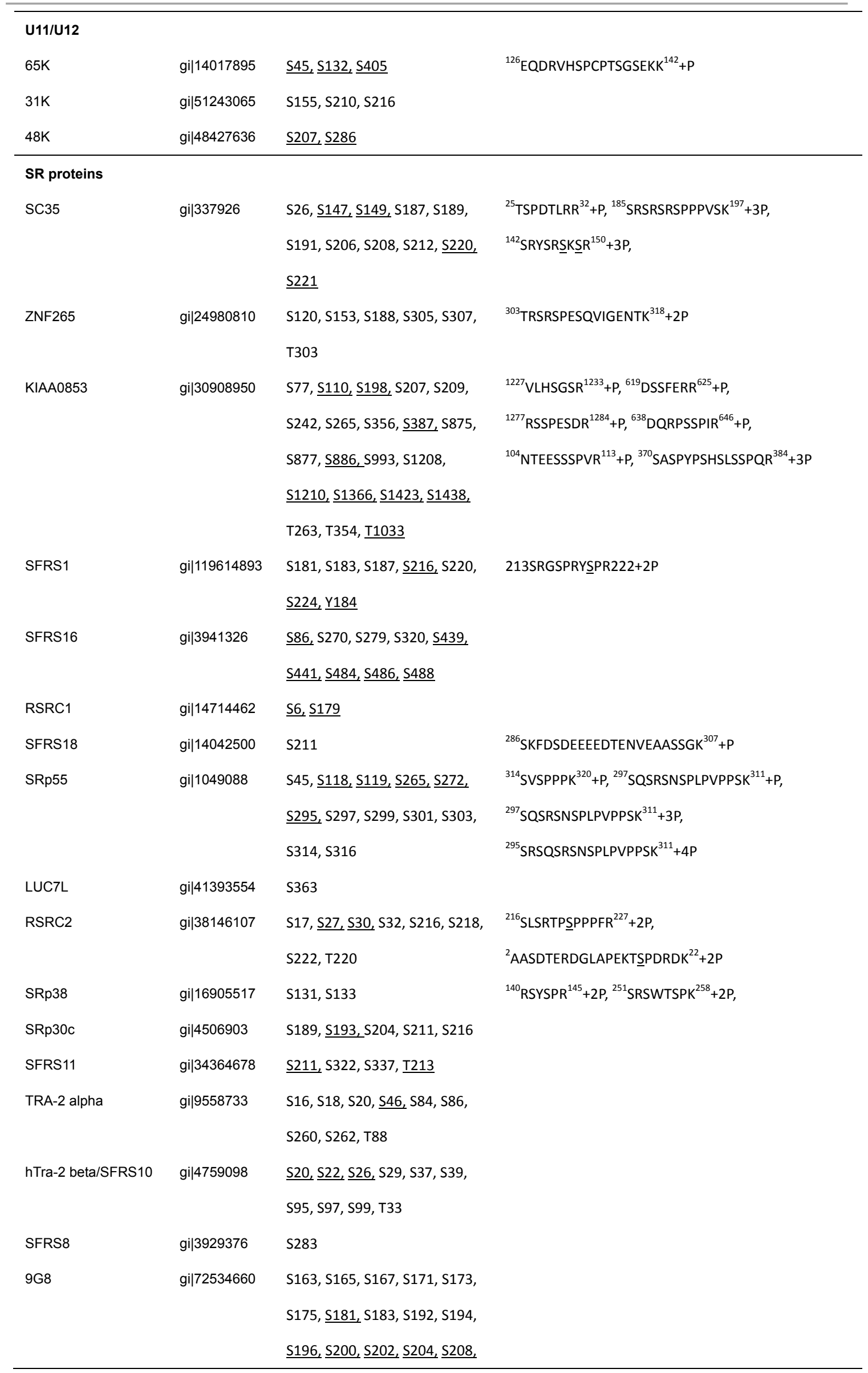




\begin{tabular}{|c|c|c|c|}
\hline & & $\underline{\mathrm{S} 209}, \underline{\mathrm{S} 215}, \mathrm{~S} 231, \mathrm{~S} 233$ & \\
\hline SRP20 & gi|4506901 & $\mathrm{S} 108, \mathrm{~S} 138, \mathrm{~S} 140, \underline{\mathrm{S} 158}, \underline{\mathrm{S} 160}$ & \\
\hline \multirow[t]{3}{*}{ SRP75 } & gi|21361282 & $\underline{\mathrm{S} 78}, \underline{\mathrm{S} 269}, \underline{\mathrm{S} 288}, \underline{\mathrm{S} 290}, \mathrm{~S} 316$ & ${ }^{482} \underline{S P S R S R S}^{489}+3 \mathrm{P}$ \\
\hline & & $\underline{\mathrm{S} 431}, \underline{\mathrm{S} 456}, \mathrm{~S} 458, \mathrm{~S} 460, \underline{\mathrm{S} 482}$, & \\
\hline & & $\underline{\mathrm{S} 488,}, \underline{\mathrm{T} 468}$ & \\
\hline CRKRS & gi|7706549 & S249, S1083, T692, T893 & \\
\hline \multirow[t]{3}{*}{ SRP40 } & gi|55640963 & $\underline{\mathrm{S} 212}, \underline{\mathrm{S} 214}, \mathrm{~S} 228, \mathrm{~S} 230, \mathrm{~S} 232$ & ${ }^{241}{ }^{\text {RGSSSRSKSPASVDR }}{ }^{255}+4 \mathrm{P}$ \\
\hline & & $\underline{\mathrm{S} 243}, \underline{\mathrm{S} 244}, \underline{\mathrm{S} 245}, \underline{\mathrm{S} 247}, \mathrm{~S} 249$ & \\
\hline & & S252 & \\
\hline SRP46 & gi|118137604 & & ${ }^{25}$ TSPDSLRR $^{32}+\mathrm{P}$ \\
\hline SRp25 & gi|7619898 & S129 & \\
\hline SR protein & gi|3253165 & $\underline{\mathrm{S} 27}, \underline{\mathrm{S} 155}, \underline{\mathrm{T} 161}, \underline{\mathrm{S} 251}$ & \\
\hline rsr-1 & gi|88952952 & $\underline{\mathrm{s} 95}, \underline{\mathrm{S} 96}$ & \\
\hline SFRS12 & $\mathrm{gi} \mid 21040255$ & & ${ }^{403}$ SSRSPSPRSR $^{412}+2 P$ \\
\hline LUC7L2 & gi|7022826 & $\underline{\mathrm{S} 281}, \underline{\mathrm{S} 283}, \underline{\mathrm{S} 336}, \mathrm{~S} 383, \mathrm{~S} 384$ & \\
\hline \multicolumn{4}{|c|}{ SR related proteins } \\
\hline \multirow[t]{22}{*}{ SRm300 } & gi|118572613 & S295, S297, S323, S351, S353, & ${ }^{1852}$ TSPAPWK ${ }^{1862}+\mathrm{P}_{,}{ }^{1693}$ SSPELTR $^{1699}+\mathrm{P}$, \\
\hline & & S377, S387, S398, S404, S408, & ${ }^{2044}$ SRSPLAIR ${ }^{2051}+\mathrm{P},{ }^{1854}$ SRTSPAPWK ${ }^{1862}+\mathrm{P}$, \\
\hline & & $\mathrm{S} 440, \underline{\mathrm{S} 456}, \underline{\mathrm{S} 484}, \underline{\mathrm{S} 508}, \underline{\mathrm{S} 510}$, & ${ }^{1457} \mathrm{HSLSGSSPGMK}^{1467}+\mathrm{P}^{778} \mathrm{SLSGSSPCPK}^{787}+2 \mathrm{P}$, \\
\hline & & $\underline{\mathrm{S} 534}, \underline{\mathrm{S} 536}, \underline{\mathrm{S} 575}, \underline{\mathrm{S} 637}, \underline{\mathrm{S} 639}$, & ${ }^{1690}$ SSRSSPELTR ${ }^{1699}+2 P,{ }^{2088}$ SRSATPPATR ${ }^{2097}+2 P$, \\
\hline & & $\underline{\mathrm{S} 702}, \underline{\mathrm{S} 704}, \underline{\mathrm{S} 706}, \mathrm{~S} 774, \mathrm{~S} 778$ & ${ }^{2407}$ SRTPPSAPSQSR $R^{2418}+P_{,}{ }^{1497}$ SRSPSSPELNNK ${ }^{1508}+3 P$, \\
\hline & & $\mathrm{S} 780, \underline{\mathrm{S} 790}, \underline{\mathrm{S} 808,}, \mathrm{~S} 820, \mathrm{~S} 857$ & ${ }^{743}$ SNSSPEMKKSRISSR ${ }^{757}+2 \mathrm{P}$, \\
\hline & & S871, S875, S876, S895, S908, & ${ }^{1001}$ SSSPVTELASRSPIR ${ }^{1115}+\mathrm{P}$, \\
\hline & & $\underline{\mathrm{S} 937,}$, S952, S954, S974, S994, & ${ }^{357}$ SSTGPEPPAPIPLLAER ${ }^{373}+2 \mathrm{P}$ \\
\hline & & $\mathrm{S} 1014, \underline{\mathrm{S} 1028}, \underline{\mathrm{S} 1054}, \mathrm{~S} 1072$ & ${ }^{1245}$ SEEPAGQILSHLSSELK $K^{1262}+\mathrm{P}$, \\
\hline & & S1073, S1083, S1101, S1102, & ${ }^{2098}{ }_{\text {NHSGSRTPPVALNSSR }}^{2113}+\mathrm{P}$ \\
\hline & & S1103, S1112, S1124, S1132, & ${ }^{433}$ HASSSPESPKPAPAPGSHR $R^{451}+3 P$, \\
\hline & & $\underline{\mathrm{S} 1181}, \underline{\mathrm{S} 1227}, \underline{\mathrm{S} 1233}, \underline{\mathrm{S} 1318,}$ & ${ }^{1539}$ SGSSQELDVKPSASPQER ${ }^{1556}+3 \mathrm{P}$ \\
\hline & & S1320, S1326, S1329, $\underline{\text { S1336, }}$ & ${ }^{1449}$ DGSGTPSRHSLSGSSPGMK ${ }^{1467}+3 P$ \\
\hline & & 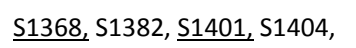 & ${ }^{979}$ VKPETPPRQSHSGSISPYPK ${ }^{998}+2 \mathrm{P}$, \\
\hline & & S1441, S1444, S1463, S1497, & ${ }^{852}$ QGSITSPQANEQSVTPQRR ${ }^{870}+2 \mathrm{P}$, \\
\hline & & S1499, S1502, S1537, S1539, & ${ }^{1138}$ FQSDSSSYPTVDSNSLLGQSR ${ }^{1158}+\mathrm{P}$, \\
\hline & & $\underline{\mathrm{S} 1541}, \underline{\mathrm{S} 1542}, \underline{\mathrm{S} 1552}, \mathrm{~S} 1592$ & ${ }^{304}$ GEGDAPFSEPGTTSTQRPSSPETATK ${ }^{329}+2 \mathrm{P}$, \\
\hline & & S1600, S1601, S1616, S1618, & ${ }^{346}$ SATRPSPSPERSSTGPEPPAPTPLLAER ${ }^{373}+2 \mathrm{P}$, \\
\hline & & S1657, S1691, S1693, S1694, & ${ }^{374}$ HGGSPQPLATTPLSQEPVNPPSEASPTR ${ }^{401}+4 \mathrm{P}$ \\
\hline & & $\underline{\mathrm{S} 1729}, \underline{\mathrm{S} 1731}, \mathrm{~S} 1842, \underline{\mathrm{S} 1854}$, & \\
\hline & & $\underline{\mathrm{S} 1857}, \underline{\mathrm{S} 1864}, \underline{\mathrm{S} 1866}, \underline{\mathrm{S} 1869}$, & \\
\hline & & S1876, S1878, S1890, S1893, & \\
\hline
\end{tabular}




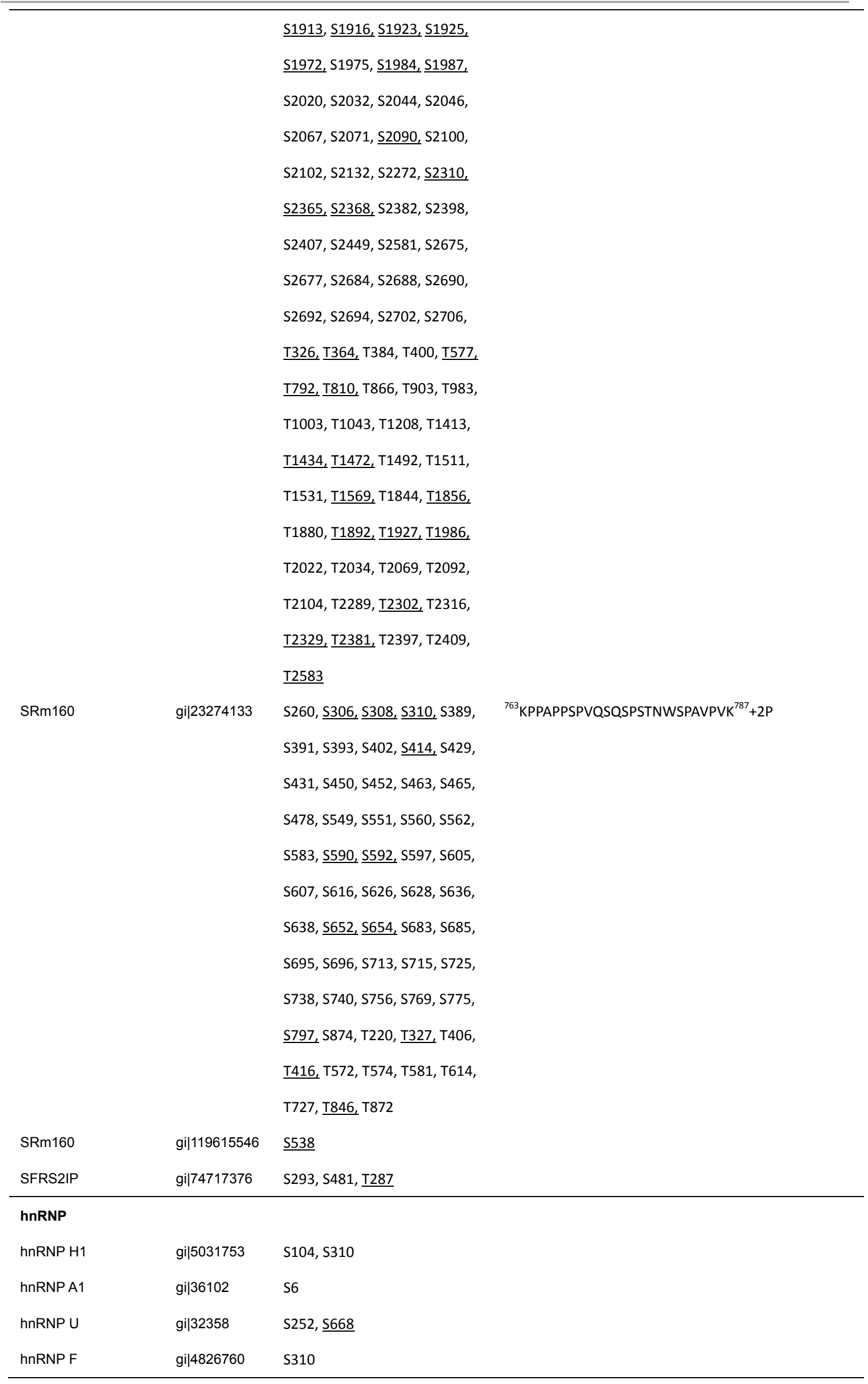


Appendixes

\begin{tabular}{|c|c|c|c|}
\hline hnRNP G & gi|3256007 & S208, S352 & ${ }^{299}$ GPPPSYGGSSR $^{309}+\mathrm{P}$ \\
\hline \multicolumn{4}{|c|}{ Lsm proteins } \\
\hline LSM11 & gi|27735089 & $\underline{\mathrm{S} 15}, \underline{\mathrm{S} 21}, \underline{\mathrm{S} 28}$ & \\
\hline Lsm3 & gi|7657315 & & ${ }^{2}$ ADDVDQQQTTNTVEEPLDLIR ${ }^{22}+P$ \\
\hline \multicolumn{4}{|c|}{ hPrp19/CDC5L } \\
\hline \multicolumn{4}{|l|}{ complex } \\
\hline \multirow[t]{6}{*}{ CDC5L } & gi|11067747 & $\underline{\mathrm{S} 64}, \mathrm{~S} 303, \underline{\mathrm{S} 317}, \underline{\mathrm{S} 393}, \underline{\mathrm{S} 437}$, & ${ }^{427}$ SGTTPKPVINSTPGR ${ }^{441}+2 \mathrm{P}$ \\
\hline & & $\underline{\mathrm{S} 463}, \underline{\mathrm{T} 61}, \underline{\mathrm{T} 355}, \underline{\mathrm{T} 373}, \underline{\mathrm{T} 377}$, & ${ }^{400} \mathrm{QVVQIIPNTVLSTPFR}^{414}+2 \mathrm{P}$ \\
\hline & & $\mathrm{T} 385, \mathrm{~T} 396, \underline{\mathrm{T} 404}, \underline{\mathrm{T} 424}, \mathrm{~T} 430$, & ${ }^{427}$ SGTTPKPVINSTPGRIPLR ${ }^{445}+2 \mathrm{P}$ \\
\hline & & $\mathrm{T} 438, \mathrm{~T} 442, \underline{\mathrm{T} 510}$ & ${ }^{362}$ ILQEAQNLMALTNVDTPLK ${ }^{380}+\mathrm{P}$ \\
\hline & & & ${ }^{427}$ SGTTPKPVINSTPGRTPLR ${ }^{445}+3 P^{427}$ SGTIPKPVINSTPGR \\
\hline & & & $\underline{T P L R}^{445}+4 \mathrm{P},{ }^{427}$ SGTTPKPVINSTPGRIPLR ${ }^{445}+5 \mathrm{P}$ \\
\hline CCAP2 & gi|6841518 & $\mathrm{T} 110, \mathrm{~S} 121, \underline{\mathrm{T} 173}$ & \\
\hline PRCC & gi|14714625 & S157, S159, $\underline{\text { S267 }}$ & ${ }^{207}{ }^{K P S D G S P D T K P S R}{ }^{219}+\mathrm{P}$ \\
\hline Npw38BP & gi|7706501 & S353, S361, S364 & \\
\hline PRL1 & gi|4505895 & & ${ }^{117}$ MPSESAAQSLAVALPLQTK ${ }^{135}+P$ \\
\hline \multicolumn{4}{|c|}{ hPrp19/CDC5L } \\
\hline \multicolumn{4}{|c|}{ related proteins } \\
\hline \multirow[t]{2}{*}{ SKIP } & gi|6912676 & $\underline{\mathrm{S} 33}, \underline{\mathrm{S} 182}, \mathrm{~S} 224, \mathrm{~S} 232, \underline{\mathrm{S} 389}$, & ${ }^{401}$ TSNEVQYDQR $^{410}+\mathrm{P}$ \\
\hline & & $\underline{\mathbf{S} 415}$ & \\
\hline hSYF1 & gi|10566459 & $\underline{\mathrm{S} 851}$ & \\
\hline CRNKL1 & gi|11055967 & $\underline{\mathrm{s} 834}$ & \\
\hline Сур-Е & gi|73917051 & $\underline{\mathrm{T} 126}$ & \\
\hline hlsy1 & gi|6330157 & S267 & \\
\hline \multicolumn{4}{|c|}{ hRES complex } \\
\hline \multicolumn{4}{|l|}{ proteins } \\
\hline \multirow[t]{2}{*}{ SNIP1 } & gi|10434110 & $\mathrm{S} 35, \underline{\mathrm{S} 52}, \underline{\mathrm{S} 54}, \underline{\mathrm{S} 74}, \underline{\mathrm{S} 76}, \mathrm{~S} 153$ & ${ }^{220}{ }^{20 K P S F E L S G A L L E D T N T F R ~}{ }^{240}+P$ \\
\hline & & S202, S394, S396 & \\
\hline \multirow[t]{8}{*}{ MGC13125 } & gi|14249338 & $\underline{\mathrm{S} 18}, \underline{\mathrm{S} 57}, \mathrm{~S} 139, \mathrm{~S} 151, \mathrm{~S} 163$, & ${ }^{170} \mathrm{HDSDTSPPR}^{178}+\mathrm{P},{ }^{121} \mathrm{HDTPDSSPR}^{129}+\mathrm{P}$, \\
\hline & & $\underline{\mathrm{S} 175}, \underline{\mathrm{S} 197}, \underline{\mathrm{S} 201}, \underline{\mathrm{S} 214}, \mathrm{~S} 222$ & ${ }^{351}$ ATDSSDLSSPR $^{360}+2 \mathrm{P}$ \\
\hline & & $\mathrm{S} 226, \underline{\mathrm{S} 235}, \underline{\mathrm{S} 240}, \mathrm{~S} 248, \underline{\mathrm{S} 258,}$ & \\
\hline & & S271, S281, S325, S354, S357, & \\
\hline & & $\underline{\mathrm{S} 358,}, \underline{\mathrm{S} 364}, \underline{\mathrm{S} 370}, \underline{\mathrm{S} 372}, \underline{\mathrm{S} 375}$ & \\
\hline & & $\underline{\mathrm{S} 385}, \underline{\mathrm{S} 386}, \underline{\mathrm{S} 388}, \underline{\mathrm{S} 391}, \underline{\mathrm{S} 401}$, & \\
\hline & & 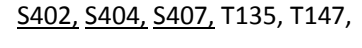 & \\
\hline & & $\mathrm{T} 159, \underline{\mathrm{T} 174}$ & \\
\hline \multirow[t]{2}{*}{ CGI-79 } & gi|118600973 & S188 & ${ }^{178}$ EVQAEQPSSSSPR $^{190}+\mathrm{P}^{229}{ }^{229}$ TAYSGGAEDLER $^{240}+\mathrm{P}$, \\
\hline & & & ${ }^{272}$ SSDAHSSWYNGR ${ }^{283}+P$ \\
\hline
\end{tabular}




\begin{tabular}{|c|c|c|c|}
\hline $\begin{array}{l}\text { Proteins recruited } \\
\text { to A-complex }\end{array}$ & & & \\
\hline tat SF1 & gi|21361437 & $\begin{array}{l}\text { S387, S579, S616, S624, S642, } \\
\text { S676, S702 }\end{array}$ & \\
\hline RBM10 & gi|1469167 & $\begin{array}{l}\underline{\mathrm{S} 170}, \underline{\mathrm{S} 803}, \mathrm{~S} 813, \mathrm{~S} 816, \mathrm{~S} 818 \\
\mathrm{~S} 877, \underline{\mathrm{S} 925}, \underline{\mathrm{Y} 812}\end{array}$ & \\
\hline Splicing factor $\mathrm{HCC} 1$ & gi|4757926 & $\begin{array}{l}\text { S97, } \underline{\mathrm{S106}}, \mathrm{S} 127, \mathrm{~S} 129, \mathrm{~S} 136, \\
\text { S337 }\end{array}$ & \\
\hline $\begin{array}{l}\text { SF1 } \\
\text { RBM5/LUCA15 }\end{array}$ & $\begin{array}{l}\text { gi|785996 } \\
\text { gi|1244404 }\end{array}$ & $\begin{array}{l}S 80, S 82 \\
S 59, \quad S 621, S 624, \underline{Y 620}\end{array}$ & \\
\hline $\begin{array}{l}\text { Proteins recruited } \\
\text { to B-complex }\end{array}$ & & & \\
\hline PRPF4B & gi|23831382 & 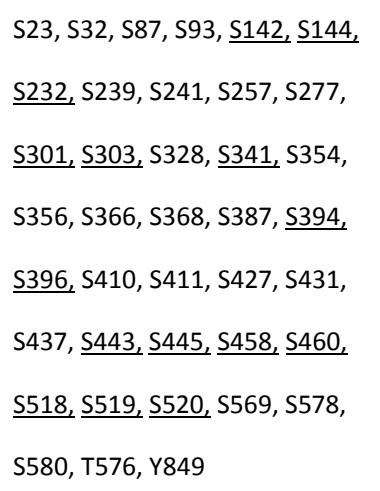 & $\begin{array}{l}{ }^{408} \text { RLSSPR }^{413}+P_{,}{ }^{332} \text { DASSGKENRSPSR }^{344}+\mathrm{P}, \\
{ }^{276} \text { ARSPTDDKVKIEDK }^{289}+\mathrm{P}, \\
{ }^{514} \text { VEQESSSDDNLEDFDVEEEDEEALIEQR }{ }^{541}+2 \mathrm{P}\end{array}$ \\
\hline GCFC & gi|22035565 & 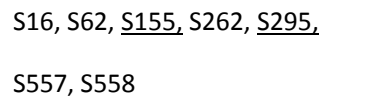 & \\
\hline THRAP3 & gi|114555524 & $\begin{array}{l}\underline{\mathrm{S} 22}, \underline{\mathrm{S} 28,}, \underline{\mathrm{S} 34}, \underline{\mathrm{S} 36}, \underline{\mathrm{S} 53}, \underline{\mathrm{S} 55,} \\
\underline{\mathrm{S} 119,} \underline{\mathrm{S} 139}, \underline{\mathrm{S} 141}, \underline{\mathrm{S} 143}, \mathrm{~S} 237, \\
\mathrm{~S} 240, \mathrm{~S} 243, \mathrm{~S} 248, \mathrm{~S} 253, \mathrm{~S} 315, \\
\mathrm{~S} 320, \mathrm{~S} 379, \mathrm{~S} 534, \mathrm{~S} 535, \mathrm{~S} 672, \\
\mathrm{~S} 682, \mathrm{~S} 928, \mathrm{~S} 939, \mathrm{~T} 874\end{array}$ & $\begin{array}{l}{ }^{922} \text { WAHDKFSGEEGEIEDDESGTENR }{ }^{944}+\mathrm{P}, \\
{ }^{803} \text { EESTTGFDK }^{811}+\mathrm{P},{ }^{203} \text { EQTFSGGTSQDTK }{ }^{215}+\mathrm{P}\end{array}$ \\
\hline MFAP1 & gi|1709012 & $\begin{array}{l}\text { S52, S53, S94, S116, S118, } \\
\text { S132, S133, T267 }\end{array}$ & \\
\hline HSPB1 & gi|4504517 & S82 & \\
\hline MATR3 & gi|9956070 & S158 & \\
\hline PRPF4B & gi|405749 & $\underline{\mathrm{S7}}, \mathrm{S9}, \mathrm{Y} 19$ & \\
\hline CCDC16 & gi|14290546 & S217 & \\
\hline KIAA1604 & gi|10047283 & $\begin{array}{l}\underline{\mathrm{S} 56}, \underline{\mathrm{S} 57}, \underline{\mathrm{S} 68,}, \underline{\mathrm{S} 90}, \underline{\mathrm{S} 93}, \underline{\mathrm{S} 120}, \\
\underline{\mathrm{S} 122}, \underline{\mathrm{S} 815}, \underline{\mathrm{S} 821}, \underline{\mathrm{S} 858}, \underline{\mathrm{S} 860}\end{array}$ & $\begin{array}{l}{ }^{127} \text { NPETSVTQSSSAQDEPATK }^{145}+\mathrm{P}, \\
{ }^{55} \text { NSSPEDRYEEQERSPR }{ }^{70}+3 \mathrm{P}, \\
{ }^{433} \text { EILDEGDTDSNTDQDAGSSEEDEEEEEEEGEEDEEGQK }^{47} \\
{ }^{0}+4 \mathrm{P}\end{array}$ \\
\hline $\mathrm{DHX} 16$ & gi|4503293 & $\mathrm{S} 103, \mathrm{~S} 106, \mathrm{~S} 107, \mathrm{~S} 160$ & \\
\hline
\end{tabular}




\begin{tabular}{|c|c|c|c|}
\hline FBP21 & gi|6005948 & $\underline{\mathrm{S} 220}, \underline{\mathrm{S} 262}, \underline{\mathrm{S} 277}$ & \\
\hline RNF113A & gi|5902158 & $\mathrm{S} 84, \mathrm{~S} 85, \mathrm{~S} 253$ & \\
\hline PPIL4 & gi|20911035 & S178 & \\
\hline RED & gi|5901878 & $\underline{\mathbf{S 4 0 9}}$ & \\
\hline $\operatorname{CCDC} 12$ & gi|21389497 & $\underline{\mathrm{S} 149}, \underline{\mathrm{S} 152}$ & \\
\hline PABPN1 & gi|4758876 & & $\begin{array}{l}{ }^{79} \text { APPGAPGPGPGSGAPGSQEEEEEPGLVEGDPGDGAIED } \\
\text { PELEAIK }^{123}+\mathrm{P}\end{array}$ \\
\hline $\begin{array}{l}\text { Proteins recruited } \\
\text { to C-complex }\end{array}$ & & & \\
\hline PPWD1 & gi|559713 & $\underline{\mathrm{S} 38}$ & \\
\hline Abstrakt & gi|21071032 & $\begin{array}{l}\mathrm{S} 21, \mathrm{~S} 23, \underline{\mathrm{S} 66}, \underline{\mathrm{S} 405}, \underline{\mathrm{S} 618}, \\
\underline{\mathrm{S} 619}\end{array}$ & \\
\hline c19orf29 & gi|122937392 & $\underline{\mathrm{S} 139}, \underline{\mathrm{S} 476}, \underline{\mathrm{T} 162}$ & \\
\hline C1orf55 & gi|21751360 & S278 & ${ }^{176}$ VVNTDHGSPEQLQIPVTDSGR ${ }^{196}+P$ \\
\hline \multicolumn{4}{|l|}{$A / B$ proteins } \\
\hline DDX9 & gi|1082769 & S87 & \\
\hline NFAR & gi|9663121 & S29 & \\
\hline NFAR & gi|1770458 & & $\begin{array}{l}{ }^{475} \text { DSSKGEDSAEETEAKPAVVAPAPVVEAVSTPSAAFPSDAT } \\
\text { AEQGPILTK }{ }^{523}+\mathrm{P}\end{array}$ \\
\hline YB-1 & gi|27807361 & S176, S314 & $\begin{array}{l}{ }^{2} \text { SSEAETQQPPAAPPAAPALSAADTKPGTTGSGAGSGGPG } \\
\text { GLTSAAPAGGDK }{ }^{52}+P\end{array}$ \\
\hline HNRPCL1 & gi|14249959 & $\underline{\mathrm{S} 220}, \underline{\mathrm{S} 240}, \underline{\mathrm{S} 247}, \underline{\mathrm{S} 286}$ & \\
\hline HNRPCL1 & gi|13937888 & $\underline{\mathrm{S} 253}, \underline{\mathrm{S} 260}$ & \\
\hline $\begin{array}{l}\text { Potential C-compl } \\
\text { specific proteins }\end{array}$ & & & \\
\hline CDC2L1 & gi|507160 & $\begin{array}{l}\underline{\mathrm{S} 47}, \underline{\mathrm{S} 232}, \underline{\mathrm{S} 262}, \mathrm{~S} 268, \mathrm{~S} 571 \\
\mathrm{~T} 577, \mathrm{~S} 734\end{array}$ & \\
\hline TOE1 (FLJ13949) & gi|10436256 & $\mathrm{S} 5, \underline{\mathrm{S} 428}$ & \\
\hline PPIG & gi|62988846 & $\begin{array}{l}\text { S71, S73, S74, S76, S107, S173, } \\
\text { S214, S504, S513, } \underline{\mathrm{S533}}, \underline{\mathrm{S} 534}, \\
\underline{\mathrm{T} 175}\end{array}$ & $\begin{array}{l}{ }^{531} \text { IRSSVEK }^{537}+\mathrm{P}, \\
{ }^{71} \text { SASSESEAENLEAQPQSTVRPEEIPPIPENR }{ }^{103}+3 P\end{array}$ \\
\hline C9orf78 & gi|7706557 & $\mathrm{S} 15, \mathrm{~S} 17, \mathrm{~S} 213$ & \\
\hline NKAP & gi|47938198 & s9, S149 & ${ }^{2} A P V S G S R S P D R E A S G S G G R^{20}+P$ \\
\hline $\mathrm{ZCCHC8}$ & gi|7018505 & $\underline{\mathrm{S} 189}, \underline{\mathrm{S} 411}, \underline{\mathrm{T} 410}$ & ${ }^{353}$ SEAGHASSPDSEVTSLCQK ${ }^{371}+P$ \\
\hline DGCR14 & gi|12804313 & $\begin{array}{l}\mathrm{S} 291, \underline{\mathrm{S} 390}, \underline{\mathrm{S} 394}, \underline{\mathrm{T} 332}, \underline{\mathrm{T} 338,} \\
\underline{\mathrm{T} 385}, \underline{\mathrm{T} 449}, \underline{\mathrm{T} 452}\end{array}$ & $\begin{array}{l}{ }^{429} \text { TPASGLQTPTSTPAPGSATRTPLTQDPASITDNLLQLPAR }{ }^{46} \\
{ }^{8}+4 \mathrm{P}\end{array}$ \\
\hline $\operatorname{CCDC} 130$ & gi|13540614 & $\underline{\mathrm{S} 286}, \underline{\mathrm{S} 306}, \underline{\mathrm{S} 362}$ & \\
\hline $\mathrm{ZCCHC} 10$ & gi|8923106 & $\underline{\mathrm{S} 68}$ & \\
\hline
\end{tabular}


Appendixes

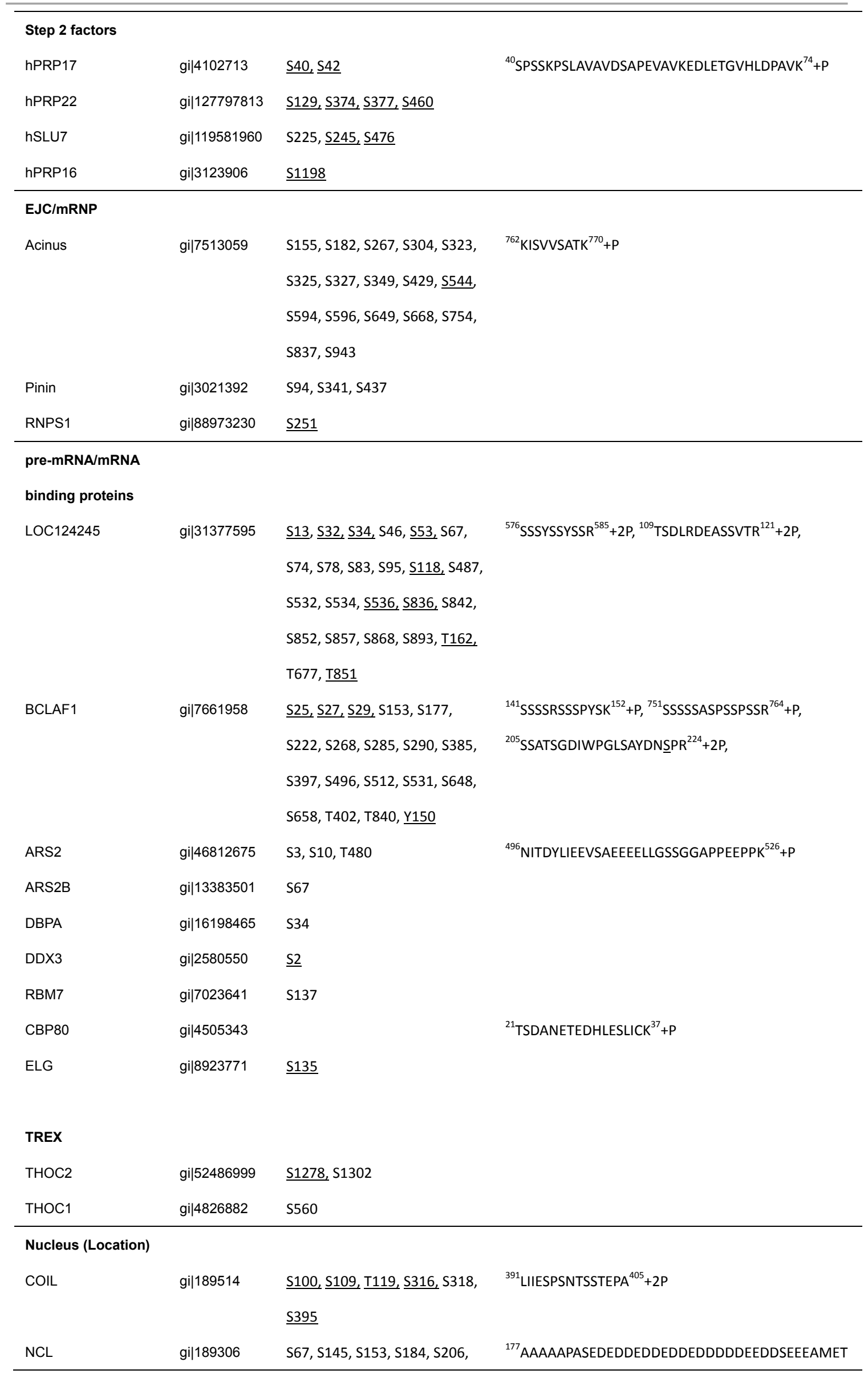




\begin{tabular}{|c|c|c|c|}
\hline & & S563 & $\mathrm{TPAK}^{217}+2 \mathrm{P}$ \\
\hline \multirow[t]{2}{*}{ ZBTB7A } & gi|7705375 & S526, S549 & ${ }^{275}$ GGEEEAASLSEAAPEPGDSPGFLSGAAEGEDGDGPDVD \\
\hline & & & GLAASTLLQQMMSSVGR ${ }^{329}+\mathrm{P}$ \\
\hline SMN1 & gi|736411 & $\mathrm{S} 27, \mathrm{~S} 30$ & \\
\hline \multirow[t]{2}{*}{ EAPP } & gi|73919272 & S17, S24, S25, S26, S109, S111, & \\
\hline & & $\underline{\mathrm{S} 175}$ & \\
\hline $\mathrm{DDX} 21$ & gi|2135315 & S107, S139 & \\
\hline DKC1 & gi|2737894 & S494, S513 & \\
\hline \multirow[t]{2}{*}{ DDX20 } & gi|5359631 & $\underline{\mathrm{S} 187}, \underline{\mathrm{S} 532}, \underline{\mathrm{S} 677}, \mathrm{~S} 678, \underline{\mathrm{S} 703}$, & \\
\hline & & S714 & \\
\hline \multirow[t]{2}{*}{ BCDIN3 } & gi|47271406 & $\mathrm{S} 60, \mathrm{~S} 69, \underline{\mathrm{T75}}, \underline{\mathrm{S} 254}, \underline{\mathrm{S} 330}$, & ${ }^{519}$ KRSCFPASLTASR $^{531}+2 \mathrm{P}$ \\
\hline & & $\underline{\mathbf{S 5 3 0}}$ & \\
\hline CCNL1 & gi|9945320 & $\mathrm{S} 65, \underline{\mathrm{S} 335}, \underline{\mathrm{S} 342}, \mathrm{~S} 352, \underline{\mathrm{S} 504}$ & ${ }^{64}{ }^{4 S P T P S M Q D G L D L P S E T D L R ~}{ }^{83}+P$ \\
\hline NPM1 & gi|825671 & S56, S111 & \\
\hline SRPK1 & gi|630737 & S51, S311 & ${ }^{346}$ DTEGGAAEINCNGVIEVINYTQNSNNETLR ${ }^{375}+\mathrm{P}$ \\
\hline \multirow[t]{2}{*}{ SAFB } & gi|1213639 & $\underline{\mathrm{T} 137}, \mathrm{~S} 293, \underline{\mathrm{S} 719}$ & ${ }^{122}$ ELPEQLQEHAIEDKETINNLDTSSSDFTILQEIEEPSLEPEN \\
\hline & & & $\mathrm{EK}^{165}+\mathrm{P}$ \\
\hline NOL5A & gi|119630989 & S580, S629, S630 & \\
\hline NOL1 & gi|12653741 & S732, S786 & \\
\hline G3BP1 & gi|5031703 & S149, S232 & \\
\hline LARP7 & gi|109809739 & $\underline{\mathrm{S} 261}, \underline{\mathrm{S} 300}, \mathrm{~S} 337, \mathrm{~T} 338$ & \\
\hline NIPBL & gi|47458031 & $\underline{\mathrm{S} 915}, \mathrm{~S} 2658$ & \\
\hline \multirow[t]{2}{*}{ SPEN } & gi|14790190 & S309, S725, S727, $\underline{\text { S1425, }}$ & \\
\hline & & $\mathrm{S} 1918, \mathrm{~S} 2120, \underline{\mathrm{S} 2412}, \underline{\mathrm{T} 2421}$ & \\
\hline DDX51 & gi|21756727 & $\mathrm{S} 83$ & \\
\hline \multirow[t]{3}{*}{ RBM15 } & gi|14041646 & $\mathrm{S} 51, \underline{\mathrm{S} 97}, \mathrm{~S} 128, \mathrm{~S} 292, \mathrm{~S} 294$ & ${ }^{257}$ SRSPLDKDTYPPSASVVGASVGGHR ${ }^{281}+2 \mathrm{P}$ \\
\hline & & S622, S656, S659, S670, S674, & \\
\hline & & S708, S741, $\underline{\mathrm{S} 655}$ & \\
\hline ARGLU1 & gi|84000355 & $\underline{\mathrm{S} 56}, \underline{\mathrm{S} 58,}, \underline{\mathrm{S} 60}, \mathrm{~S} 77$ & \\
\hline SAP30BP & gi|9994179 & $\underline{\mathrm{S} 22}$ & ${ }^{6}$ NVLSSLAVYAEDSEPESDGEAGIEAVGSAAEEK ${ }^{38}+2 \mathrm{P}$ \\
\hline TAF15 & gi|4507353 & & ${ }^{219}$ TDADSESDNSDNNTIFVQGLGEGVSTDQVGEFFK ${ }^{252}+\mathrm{P}$ \\
\hline UBR5 & gi|15147337 & S1549 & \\
\hline KPNA3 & gi|1934907 & $\mathrm{S} 60$ & \\
\hline NOLC1 & gi|4758860 & $\mathrm{S} 698$ & \\
\hline SSRP1 & gi|4507241 & S444 & \\
\hline $\mathrm{HD}$ & gi|4586876 & $\underline{\mathrm{S} 1261}$ & \\
\hline RBM14 & gi|5454064 & $\underline{\mathrm{S} 147}, \underline{\mathrm{T} 148,}, \mathrm{~T} 206$ & ${ }^{263}$ TQPMTAQAASYR $^{374}+\mathrm{P}$ \\
\hline SP1 & gi|339518 & $\underline{\mathrm{S} 523}$ & \\
\hline
\end{tabular}


Appendixes

\begin{tabular}{|c|c|c|c|}
\hline $\mathrm{ZC} 3 \mathrm{H} 14$ & gi|28207879 & $\underline{\mathbf{S 4 3 0}}$ & \\
\hline TOP2A & gi|105857 & S1376, S1392 & \\
\hline KIAA1429 & gi|7243239 & S171, S220, S1577 & \\
\hline MPHOSPH10 & gi|2230873 & S233 & \\
\hline $\mathrm{CHD8}$ & gi|34328020 & $\underline{\mathrm{T} 1189}$ & \\
\hline NEK2 & gi|62898267 & $\underline{\mathrm{S} 354}, \underline{\mathrm{Y} 355}$ & \\
\hline GEMIN8 & gi|8923481 & & ${ }^{112}$ EDQALSKEEEMETESDAEVECDLSNMEITEELR ${ }^{144}+2 \mathrm{P}$ \\
\hline MLF2 & gi|4885487 & S238 & \\
\hline NOC2L & gi|7512721 & S54 & \\
\hline TSPYL2 & gi|11545835 & $\underline{\mathbf{S 2 0 4}}$ & \\
\hline ZNF788 & gi|34535580 & $\underline{\mathrm{T} 254}$ & \\
\hline PRKRIP1 & gi|13375901 & & ${ }^{159}$ EQGSSSSAEASGTEEEEEVPSFTMGR $^{184}+\mathrm{P}$ \\
\hline NPM1 & gi|63101692 & S42, S61 & \\
\hline SSB & gi|337457 & S313 & \\
\hline ZBTB11 & gi|7657703 & $\underline{Y 319}$ & \\
\hline ZNF167 & gi|10434411 & $\underline{\mathrm{T} 157}, \underline{\mathrm{T} 161}$ & \\
\hline GEMIN5 & gi|22001417 & $\mathrm{S} 778, \underline{\mathrm{Y} 416}$ & \\
\hline CROP & gi|7023491 & S333, S335, S367, S425, S431 & \\
\hline YTHDC1 & gi|16551831 & S95, S257, S446, T97 & ${ }^{66}$ LSSSASREPYK $^{76}+P$ \\
\hline CLK3 & gi|4502885 & $\underline{\mathbf{S 1 3 5}}$ & ${ }^{78}$ SPSFGEDYYGPSR $^{88}+P$ \\
\hline CLK1 & gi|1705918 & S140 & ${ }^{323}$ VVDFGSATYDDEHHSTLVSTR ${ }^{343}+2 \mathrm{P}$ \\
\hline JARID1D & gi|1871160 & $\underline{\mathrm{S} 114}, \underline{\mathrm{S} 116}$ & \\
\hline RNF8 & gi|34304336 & $\underline{\mathbf{s} 331}$ & \\
\hline NOL5 & gi|6841462 & S438 & \\
\hline ANP32E & gi|119573964 & $\underline{\mathrm{T} 243}$ & \\
\hline MGEA5 & gi|10645186 & $\underline{\mathrm{S} 485}$ & \\
\hline BLM & gi|4557365 & $\underline{\mathrm{T} 321}, \underline{\mathrm{S} 328}$ & \\
\hline OTUD7B & gi|9367763 & & ${ }^{30}$ STGAEPGLAR ${ }^{39}+P$ \\
\hline SLC4A1AP & gi|8922557 & S466 & \\
\hline GTF2B & gi|254934 & & ${ }^{49}$ TFSNDKATKDPSR $^{61}+P$ \\
\hline \multirow[t]{2}{*}{ RBM15B } & gi|54607124 & $\underline{\mathrm{S} 109}, \underline{\mathrm{S} 265}, \underline{\mathrm{S} 267}, \mathrm{~S} 552, \mathrm{~S} 562$ & \\
\hline & & S609 & \\
\hline ZC3H7B & gi|119580830 & & ${ }^{64}$ QSPACPTLPCSSR ${ }^{76}+\mathrm{P}$ \\
\hline HOXB3 & gi|123268 & $\underline{\mathrm{S} 181}$ & \\
\hline AHR & gi|6330736 & & ${ }^{476}$ NSPISHPPSPSPSAYSSR ${ }^{493}+3 P$ \\
\hline ZNF264 & gi|4585643 & $\underline{\mathrm{T} 163}$ & \\
\hline RFC1 & gi|296908 & S69, S71 & \\
\hline NUP98 & gi|4545099 & $\underline{\mathrm{S} 1312}$ & \\
\hline
\end{tabular}


Appendixes

\begin{tabular}{|c|c|c|c|}
\hline FIP1L1 & gi|15079403 & S226 & \\
\hline DEK & gi|4503249 & $\begin{array}{l}\text { S32, S227, S230, S231, S232, } \\
\text { S243, S244, S251, S301, S303, } \\
\text { S306, S307 }\end{array}$ & ${ }^{299}$ KESESEDSSDDEPLIK ${ }^{314}+3 P$ \\
\hline PRPF40A & gi|5360087 & S347, S397 & ${ }^{385}$ DSGNWDTSGSELSEEGELEK $K^{403}+2 P$ \\
\hline NUMA1 & gi|35121 & S1743 & \\
\hline WTAP & gi|10334526 & S303 & \\
\hline PRPF38B & gi|8922358 & S527, S529 & \\
\hline SMC4 & gi|6807671 & S41, $\underline{\mathbf{S 3 5 9}}$ & \\
\hline MED24 & gi|8699628 & S862 & \\
\hline TRIM28 & gi|5032179 & & ${ }^{33}$ STAPSAAASASASAAASSPAGGGAEALELLEHCGVCR ${ }^{69}+\mathrm{P}$ \\
\hline YY1 & gi|186768 & S118 & \\
\hline SUB1 & gil|62088150 & $\begin{array}{l}\text { S16, S17, S18, S19, S20, S24, } \\
\underline{\text { S125 }}\end{array}$ & ${ }^{13}$ ELVSSSSSSGSDSDSEVDKK ${ }^{31}+6 P$ \\
\hline TCEA1 & gi|313223 & S100 & \\
\hline PDS5B & gi|7657269 & $\underline{\mathrm{S} 1358}$ & \\
\hline INTS1 & gi|7243278 & & ${ }^{513}$ SSPEQPIGQGR ${ }^{523}+P$ \\
\hline DMAP1 & gi|7243231 & T473 & \\
\hline TCOF1 & gi|1587477 & S1284 & \\
\hline ZNF91 & gi|549839 & $\underline{\mathrm{T} 1038}, \underline{\mathrm{T} 1039}$ & \\
\hline POLR1A & gi|49256401 & $\underline{\mathbf{s 9 3 1}}$ & \\
\hline DBP5 (DDX19) & gi|17046381 & $\begin{array}{l}\text { S154, S283, } \underline{\mathrm{S} 1002,} \underline{\mathrm{S1556},} \\
\mathrm{S} 1697, \mathrm{~S} 1948, \mathrm{~S} 1950, \underline{\mathrm{S1952},} \\
\text { S2009, S2011, S2013 }\end{array}$ & \\
\hline BAT2 & gi|18375626 & S1089, S1219 & ${ }^{597}$ EGPEPPEEVPPPTTPPVPK ${ }^{615}+P$ \\
\hline ZC3HAV1 & gi|119604309 & S19 & \\
\hline AKAP8 & gi|5031579 & S323, S328, S339 & \\
\hline EXOsc9 & gi|179287 & $\underline{\mathrm{S} 222}$ & \\
\hline IGF2BP1 & gi|56237027 & $\underline{\mathrm{S} 181}$ & \\
\hline SAF-A2 & gi|52545896 & $\mathrm{S} 40, \mathrm{~S} 107, \underline{\mathrm{T} 44}$ & \\
\hline SAFB & gi|62244004 & $\mathrm{S} 271, \mathrm{~S} 535, \mathrm{~S} 572, \underline{\mathrm{S} 730}$ & \\
\hline CARF & gi|8923040 & $\underline{\mathrm{S} 10}, \underline{\mathrm{Y} 8}$ & ${ }^{378}$ SSSQTSGSLVSK $^{386}+\mathrm{P},{ }^{161}$ TSAKTER $^{167}+\mathrm{P}$ \\
\hline CLK4 & gi|10190706 & $\mathrm{S} 138, \underline{\mathrm{S} 140}$ & \\
\hline RBMY1F & gi|113430736 & $\underline{\mathrm{S} 164}$ & \\
\hline ZNF638 & gi|1374698 & $\underline{\mathrm{S} 490}, \underline{\mathrm{S} 1401}, \underline{\mathrm{T} 1487}$ & \\
\hline SAFB2 & gi|7661936 & $\underline{\mathbf{S} 287}$ & ${ }^{189}$ NTLETSSLNFK $^{199}+P$ \\
\hline PPHLN1 & gi|7020106 & S21 & \\
\hline ESR2 & gi|3091286 & & ${ }^{133}$ CASPVTGPGSK ${ }^{143}+\mathrm{P},{ }_{1}^{199} \mathrm{KSCQACR}^{205}+\mathrm{P}$ \\
\hline
\end{tabular}




\begin{tabular}{|c|c|c|c|}
\hline UTY & gi|2580576 & & ${ }^{380}$ RCSNTSTLAAR $^{390}+P$ \\
\hline DDX50 & gi|16551055 & $\underline{\mathrm{S} 48}$ & \\
\hline HIST1H2AD & gi|89037003 & & ${ }^{120}$ KTESHLK $^{126}+\mathrm{P}$ \\
\hline FRA10AC1 & gi|14189976 & $\mathrm{S} 151, \underline{\mathrm{S} 156}, \mathrm{~S} 161$ & ${ }^{155} \mathrm{~N} \underline{\mathrm{S}} \mathrm{DEEES}$ ASESELWK ${ }^{169}+3 P$ \\
\hline WIZ & gi|4056411 & & ${ }^{124}$ TPLALAGSPTPK $^{135}+P$ \\
\hline ZNF136 & gil4507987 & $\underline{\mathrm{S} 292}, \underline{\mathrm{T} 294}$ & \\
\hline TGS1 & gi|14278850 & $\underline{\mathrm{S} 85, \mathbf{S 8 9}}$ & \\
\hline MYCBP2 & gi|3319326 & $\underline{\mathbf{S} 2797}$ & ${ }^{2794}$ SSPSGASSPR $^{2803}+2 \mathrm{P},{ }^{2766} \mathrm{MPSSSRAESPGPGSR}^{2780}+\mathrm{P}$ \\
\hline TTN & gi|1212992 & $\underline{\mathrm{S} 16047}, \underline{\mathrm{T} 18743}, \underline{\mathrm{T} 18744}$ & \\
\hline CCNL2 & gi|33150646 & $\underline{\mathrm{S} 15}, \mathrm{~S} 46, \underline{\mathrm{S} 102}, \underline{\mathrm{S} 104}$ & \\
\hline TSEN54 & gi|31565520 & & ${ }^{203} \mathrm{RSSSSPR}^{209}+2 \mathrm{P}$ \\
\hline BIVM & gi|7020076 & $\underline{\mathrm{S} 254}, \underline{\mathrm{S} 255}$ & \\
\hline CASZ1 & gi|78191040 & & ${ }^{1550}$ QFSSSADCAVPDCK ${ }^{1563}+P$ \\
\hline HN1L & gi|21700763 & $\underline{\mathrm{S} 188}$ & \\
\hline SNRPN & gi|36495 & $\underline{18}$ & \\
\hline RANBP2 & gi|857368 & $\underline{\mathrm{S} 778}, \underline{\mathrm{T779}}$ & \\
\hline BAT2D1 & gi|5541863 & S1249, S2105 & \\
\hline \multicolumn{4}{|l|}{ Membrane } \\
\hline \multicolumn{4}{|l|}{ (Location) } \\
\hline NKTR & gi|6631100 & $\underline{\mathrm{S} 463}, \underline{\mathrm{S} 613}, \underline{\mathrm{S} 1077}, \underline{\mathrm{S} 1146}$ & \\
\hline PARD3 & gi|13491612 & $\underline{\mathrm{S} 201}$ & ${ }^{466}$ KGTEGLGFSITSR $^{478}+\mathrm{P}$ \\
\hline SEPT9 & gi|5106557 & S12 & \\
\hline MFI2 & gi|136204 & $\underline{\mathrm{S} 51}$ & \\
\hline CLEC4E & gi|7657333 & & ${ }^{6}$ SSETQCTER $^{14}+P$ \\
\hline SDK1 & gi|32351274 & $\underline{\mathrm{S522}}$ & \\
\hline EXT1 & gi|1168162 & & ${ }^{127}$ IAESYQNILAAIEGSR ${ }^{142}+2 P$ \\
\hline ABCB10 & gi|9961244 & $\underline{\mathrm{S} 34}$ & \\
\hline ABCA7 & gi|119589965 & $\underline{\mathrm{S} 1255}$ & \\
\hline KCNS2 & gil6329973 & $\underline{\mathbf{S} 475}$ & \\
\hline ARTS1 & gi|6381989 & & ${ }^{714}$ QTWTDEGSVSERMLR ${ }^{728}+P$ \\
\hline SIGLEC15 & gi|119621867 & & ${ }^{468}$ RCNVVCATSPER $^{479}+\mathrm{P}$ \\
\hline BCL2L7P1 & gi|2493275 & $\underline{\mathrm{S} 3}$ & \\
\hline ENPP1 & gi|119568426 & $\underline{\mathrm{T707}}, \underline{\mathrm{T} 713}$ & \\
\hline NDUFB3 & gi|4505361 & $\underline{\mathrm{T} 27}$ & \\
\hline TAPT1 & gi|22759976 & $\underline{Y 99}$ & \\
\hline KIR3DL1 & gi|995757 & $\underline{\mathrm{S} 243}, \underline{\mathrm{S} 245}, \underline{\mathrm{S} 246}$ & \\
\hline KIAA0716 & gi|21757978 & $\underline{\mathbf{S 1 2 1}}$ & \\
\hline GPRIN1 & gi|21739648 & $\underline{\mathrm{S} 660}, \underline{\mathrm{S} 663}$ & \\
\hline
\end{tabular}




\begin{tabular}{|c|c|c|c|}
\hline TMEM61 & gi|32698902 & $\underline{s 92}$ & \\
\hline RAB43 & gi|50234889 & $\underline{\mathrm{S} 166}, \underline{\mathrm{S} 167}$ & \\
\hline ALG11 & gi||34535907 & $\underline{\mathrm{S} 169}, \underline{\mathrm{S} 172}$ & \\
\hline \multicolumn{4}{|l|}{ Cytoplasm } \\
\hline \multicolumn{4}{|l|}{ (Location) } \\
\hline BSN & gi|2662149 & $\underline{\mathrm{S} 634}$ & \\
\hline ABLIM1 & gi|57162149 & s96 & \\
\hline AMBRA1 & gi|10435015 & & ${ }^{533}$ SSERPGTSR $^{541}+P$ \\
\hline ARHGDIA & gi|36038 & & ${ }^{179}$ SRFTDDDK $^{186}+P$ \\
\hline HSP90AB1 & gi|306891 & S255 & \\
\hline CAB39 & gi|42543739 & $\underline{\mathrm{T} 40}, \underline{\mathrm{Y} 50}$ & \\
\hline SPTBN1 & gi|119620543 & S2016, $\underline{\text { S2255 }}$ & \\
\hline CTTN & gi|2498954 & S418 & ${ }^{399}$ TQTPPVSPAPQPTEERLPSSPVYEDAASFK ${ }^{428}+\mathrm{P}$ \\
\hline FXR1 & gi|1730139 & $\underline{\mathrm{T} 5}$ & \\
\hline DBN1 & gi|2498313 & S142 & \\
\hline DSP & gi|1147813 & $\underline{\mathrm{S} 333}$ & \\
\hline SHB & gi|406738 & & ${ }^{448}$ QSSPSPSR ${ }^{455}+P$ \\
\hline DIXDC1 & gi|12698015 & T436 & \\
\hline SPTBN5 & gi|17369320 & $\underline{\mathrm{S} 766}, \underline{\mathrm{S} 767}, \underline{\mathrm{S} 2501}, \underline{\mathrm{S} 3052}$ & \\
\hline \multicolumn{4}{|l|}{ Others } \\
\hline WDR79 & gi|8922396 & S54, S85, S90, S491 & \\
\hline \multirow[t]{3}{*}{ GPATCH8 } & gi|50962882 & $\underline{\mathrm{S} 653}, \underline{\mathrm{S} 740}, \underline{\mathrm{S} 758}, \underline{\mathrm{S} 898}, \underline{\mathrm{S} 981}$, & \\
\hline & & S1009, S1014, S1033, S1035, & \\
\hline & & S1107 & \\
\hline LMO7 & gi|17225574 & S533, S654, S657, S692, S1259 & \\
\hline \multirow[t]{2}{*}{ PRSAP2 } & gi|89059414 & $\underline{\mathrm{S}} 472, \underline{\mathrm{T} 626}$ & ${ }^{619}{ }^{K C R T G S M T H G L K}{ }^{630}+\mathrm{P}$ \\
\hline & & & ${ }^{1771}$ ASTXASTATASRTMRXK ${ }^{1787}+\mathrm{P}$ \\
\hline FNBP4 & gi|6808095 & $\mathrm{S} 16, \underline{\mathrm{S} 112}, \underline{\mathrm{S} 427}, \underline{\mathrm{S} 460}$ & \\
\hline RPLP1 & gi|4506669 & S101, S104 & \\
\hline CCNK & gi|8980825 & S324, S340 & ${ }^{322} \mathrm{KPSPQPSSPR}^{331}+2 \mathrm{P}$ \\
\hline TNRC15 & gi|7023190 & S26 & \\
\hline SEPT2 & gi|1040689 & S218 & \\
\hline PLEC1 & gi|41322923 & & ${ }^{19}$ TSSEDNLYLAVLR $^{31}+\mathrm{P}$ \\
\hline BCR & gi|44238463 & $\underline{\mathrm{S} 60}$ & \\
\hline AFAP1L1 & gi|10441465 & $\underline{\mathrm{S} 668}$ & \\
\hline LOC729639 & gi|113425854 & $\underline{\mathrm{S} 348}$ & \\
\hline LOC731115 & gi|113429485 & $\underline{\mathrm{T}}$ & \\
\hline GRHPR & gi|10439099 & $\underline{\mathrm{S} 192}$ & \\
\hline
\end{tabular}




\begin{tabular}{|c|c|c|c|}
\hline SPECC1L & gi|2280485 & & ${ }^{524}$ ETERSDMK $^{531}+\mathrm{P}$ \\
\hline KIAA1783 & gi|89043071 & $\underline{\mathrm{S} 279}$ & \\
\hline INPP5B & gi|1019103 & $\underline{\mathrm{S} 163}, \underline{\mathrm{T} 165}$ & \\
\hline TRIML1 & gi|31542779 & $\underline{\mathrm{T} 275}$ & \\
\hline hCG1820449 & gi|28207851 & $\underline{\mathrm{s} 87}, \underline{\mathrm{s} 88}$ & \\
\hline hCG1654449 & gi|119602732 & $\underline{\mathrm{S} 10}$ & \\
\hline ANKRD26 & gi|34532267 & $\underline{Y 34}, \underline{Y 38}$ & \\
\hline SPARCL1 & gi|21359871 & $\underline{\mathrm{T} 628}$ & \\
\hline hCG2036616 & gi|88989580 & $\underline{\mathrm{T} 95}$ & \\
\hline LOC400870 & gi|46409568 & $\underline{\mathrm{s} 92}$ & \\
\hline FAM112B & gi|21389379 & $\underline{\mathrm{T} 50}$ & \\
\hline LOC729518 & gi|113426178 & $\underline{\mathrm{s} 88}$ & \\
\hline TTC28 & gi|16741254 & $\underline{\mathrm{S} 1073}$ & \\
\hline GPATCH1 & gi|21361684 & $\mathrm{s} 6, \underline{\mathrm{s} 8}$ & \\
\hline hCG2025834 & gi|119603486 & $\underline{\mathrm{S} 148}$ & \\
\hline TSSC4 & gi|4567068 & $\underline{\mathrm{S} 132}$ & ${ }^{319}$ SSSPEDPGAEV ${ }^{329}+\mathrm{P}$ \\
\hline YME1L1 & gi|7657689 & $\underline{\mathrm{S} 163}$ & \\
\hline EEF1D & gi|38522 & $\underline{\mathrm{T} 256}$ & \\
\hline $\mathrm{ZCCHC} 3$ & gi|23396534 & $\underline{\mathrm{T} 3}$ & \\
\hline TTTY12 & gi|27805759 & $\underline{\mathrm{S} 78}$ & \\
\hline TNS1 & gi|111599618 & $\underline{\mathrm{T} 1506}$ & \\
\hline hCG22052 & gi|119568106 & $\underline{\mathrm{S} 156}, \underline{\mathrm{Y} 157}, \underline{\mathrm{S} 159}$ & \\
\hline KRI1 & gi|10434729 & S59 & \\
\hline LIMCH1 & gi|20521766 & $\mathrm{S} 736$ & \\
\hline hCG1817914 & gi|119571393 & & ${ }^{632}$ SGSTGSLKHLR ${ }^{642}+P$ \\
\hline RPS3 & gi|32532 & $\mathrm{T} 221$ & \\
\hline NRD1 & gi|2462485 & $\underline{\mathrm{S} 106}, \underline{\mathrm{Y} 113}$ & \\
\hline LOC731726 & gi|113422156 & $\underline{\mathrm{T} 132}$ & \\
\hline hCG1993567 & gi|119570834 & & ${ }^{1}$ MVELIFIPTNSDSR $^{14}+P$ \\
\hline $\mathrm{ZC} 3 \mathrm{H} 4$ & gi|4884368 & S283 & \\
\hline C9orf131 & gi|6599155 & $\underline{\mathrm{T} 102}$ & \\
\hline MMTAG2 & gi|13236559 & & ${ }^{210}$ RPAEATSSPTSPERPR ${ }^{225}+\mathrm{P}$ \\
\hline TMOD4 & gi|21751358 & $\underline{\mathrm{T} 118}$ & \\
\hline myeloid/lymphoid & gi|13376343 & & ${ }^{33} \mathrm{SSPIPPFSSR}^{42}+\mathrm{P}$ \\
\hline hCG2038818 & gi|119622863 & $\underline{\mathbf{S} 135}$ & \\
\hline RPL14 & gi|1710488 & S139 & \\
\hline C12orf11 & gil6807949 & S59 & \\
\hline C17orf47 & gi|34194295 & $\underline{\mathrm{S} 483}, \underline{\mathrm{S} 486}$ & \\
\hline
\end{tabular}


Appendixes

\begin{tabular}{|c|c|c|c|}
\hline TCTEX1D4 & gi|61966721 & $\underline{\mathbf{S} 24}$ & \\
\hline EIF3C & gi|1931584 & S39 & \\
\hline CCDC131 & $\mathrm{gi} \mid 29421174$ & S37, S958 & \\
\hline TUBA1C & gi|340021 & S48, S439 & \\
\hline USP42 & gi|51094456 & $\mathrm{S} 856, \underline{\mathrm{S} 936}, \underline{\mathrm{S} 1263}$ & ${ }^{747}$ DAEPQPGSPAAESLEEPDAAAGLSSTK ${ }^{773}+P$ \\
\hline \multirow[t]{4}{*}{ RBBP6 } & gi|15705403 & S340, S554, S582, S584, S594, & \\
\hline & & S596, S685, S697, S1003, & \\
\hline & & $\underline{\mathrm{S} 1045}, \mathrm{~S} 1101, \mathrm{~S} 1152, \underline{\mathrm{S} 1449}$, & \\
\hline & & $\underline{\mathrm{T} 1292}$ & \\
\hline PCYT1B & gi|3153239 & $\underline{\mathbf{S 2 7 1}}$ & \\
\hline hCG1993516 & gi|119593833 & $\underline{\mathrm{S} 42}, \underline{\mathrm{S} 44}$ & \\
\hline BOK & gi|6456033 & $\underline{\mathrm{T} 69}$ & \\
\hline EIF3B & gi|1778051 & & ${ }^{142}$ ALENGDADEPSFSDPEDFVDDVSEEELLGDVLK ${ }^{174}+\mathrm{P}$ \\
\hline EIF5 & gi|1229140 & S389, S390 & \\
\hline CDC2L5 & gi|14110387 & S317, S325 & \\
\hline RBM12B & gi|118722349 & $\underline{\mathrm{S} 638}$ & ${ }^{226} \mathrm{SSSPIPR}^{232}+\mathrm{P}$ \\
\hline SFRP4 & gi|4506895 & & ${ }^{226} \mathrm{SSSPIPR}^{232}+\mathrm{P}$ \\
\hline C19orf34 & gi|109452617 & & ${ }^{16}$ ATVITTR R $^{22}+\mathrm{P}$ \\
\hline TUBB5 & gi|18088719 & $\underline{\mathrm{S} 172}$ & \\
\hline DHX34 & gi|38158022 & $\underline{\mathrm{S} 749}, \underline{\mathrm{S} 750}$ & \\
\hline HSPA2 & gi|4204880 & $\underline{\mathrm{T} 164}$ & \\
\hline ZNHIT3 & gi|703110 & S77 & \\
\hline S22900 & gi|107784 & $\underline{\mathrm{s} 5}$ & \\
\hline SGIP1 & gi|51476757 & T227 & \\
\hline BAB15370 & gi|10438882 & $\underline{\mathrm{S} 4}, \underline{\mathrm{S} 6}$ & \\
\hline hCG1981358 & gi|119602855 & $\underline{\mathrm{S} 3}, \underline{\mathrm{T} 4}$ & \\
\hline EXOC1 & gi|7023220 & $\underline{\mathrm{Y} 400}$ & \\
\hline FGA & gi|223918 & S3 & \\
\hline FBXO10 & gi|6103649 & $\underline{\mathrm{S} 127,}, \underline{\mathrm{S} 464}$ & ${ }^{202}{ }^{K R S S S S P R}{ }^{209}+P$ \\
\hline CXorf40A & gi|31377535 & $\underline{\mathrm{S} 32}, \underline{\mathrm{S} 125}, \underline{\mathrm{T} 122}$ & \\
\hline PIK3AP1 & gi|20987486 & $\underline{\mathrm{S} 241}$ & \\
\hline SYBU & gi|50949414 & $\underline{\mathrm{S} 52}, \underline{\mathrm{S} 55}$ & \\
\hline LCA10 & gi|116517307 & $\underline{\mathrm{T} 97}$ & \\
\hline SNX32 & gi|16550234 & $\underline{\mathbf{S 2 5 0}}$ & \\
\hline C1orf74 & gi|22749019 & $\underline{\mathrm{S} 125}, \underline{\mathrm{S} 128}$ & \\
\hline ZC3H6 & gi|34528944 & $\underline{\mathrm{T} 313}$ & \\
\hline BAC85541 & gi|34528618 & $\underline{\mathrm{S}}, \underline{\mathrm{SZ}}$ & \\
\hline HARSL & gi|15029520 & S161 & \\
\hline
\end{tabular}


Appendix 2. MS and MS/MS spectra of phosphopeptides derived from human PRP6 (A-C) and PRP31 (D-G). The experimentally measured precursor masses and the charge states are listed in the MS spectra (small boxes). The sequences of the phosphopeptides are listed within the MS/MS spectra. Selected $y$ - and b-type ions that led to the identification of the actual phosphorylation sites are indicated within the sequences and at the corresponding fragment peaks in the MS/MS spectra. Unambiguously identified phosphorylation sites are listed as pS, pT and pY, potentially phosphorylated amino acids are in small letters $(s, t, y)$. Neutral loss of phosphoric acid (-98) of $y$ - and/or b-type ions is indicated within the sequences and MS/MS spectra

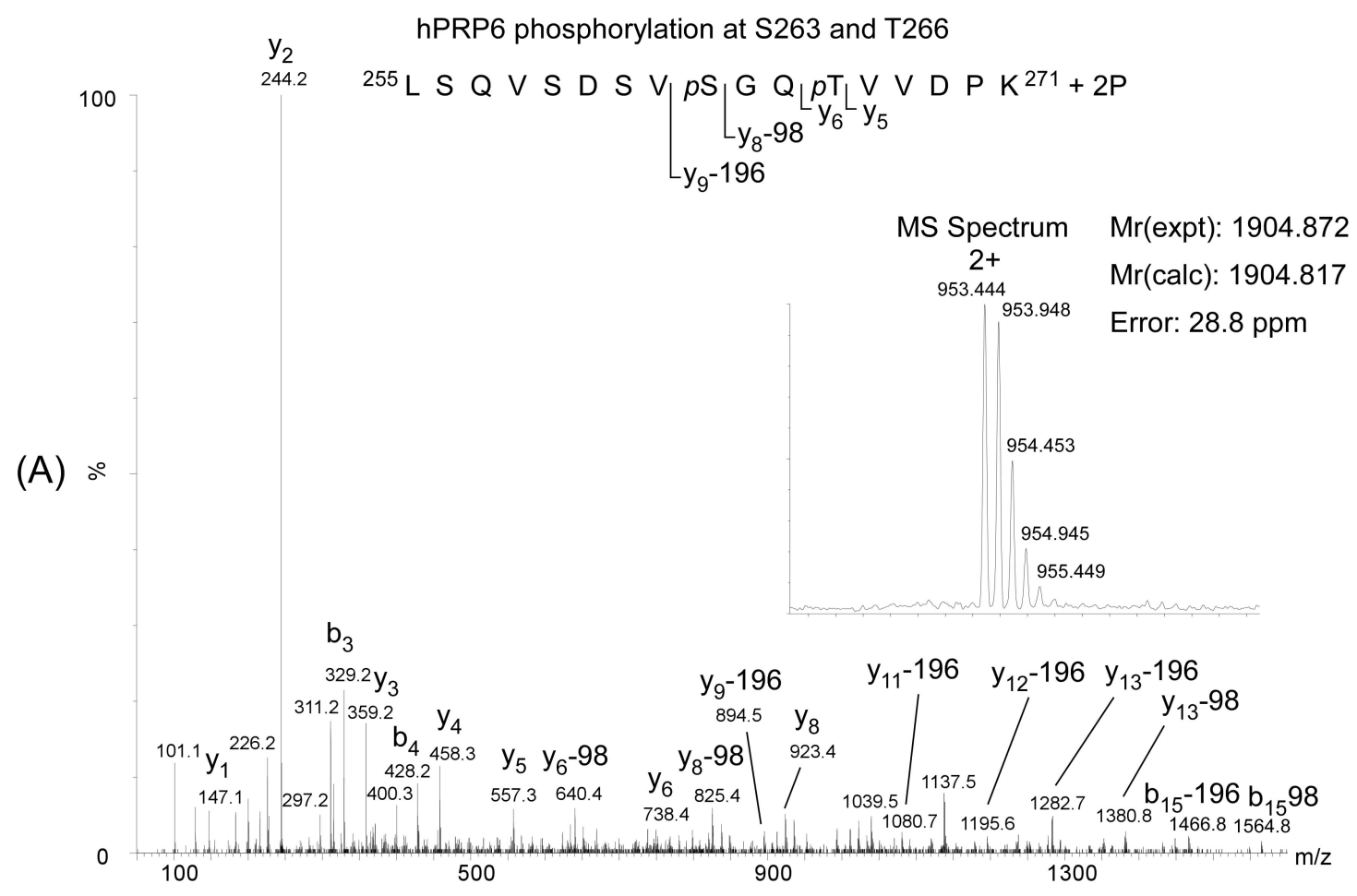



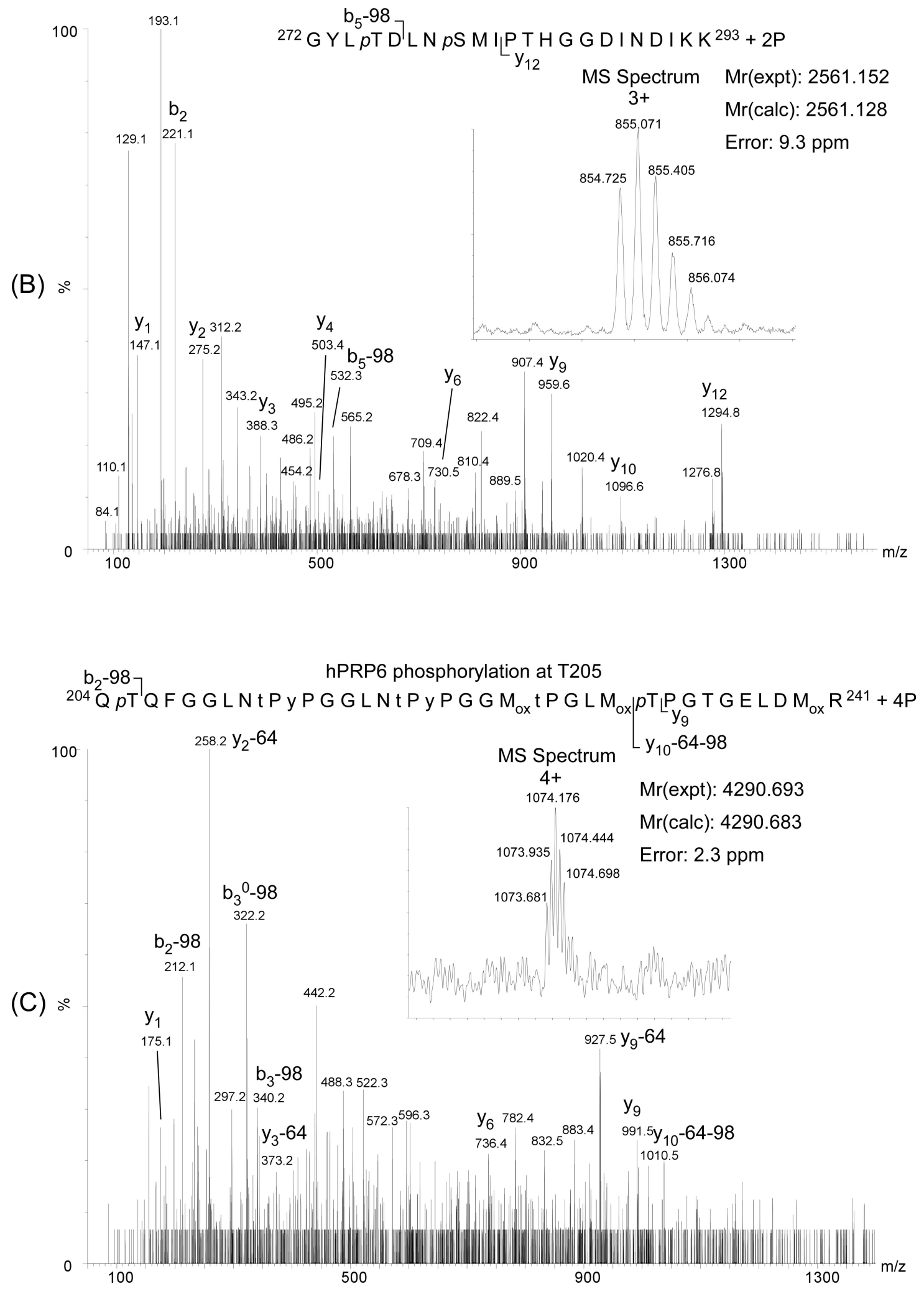
hPRP31 phosphorylation at S439 and T440
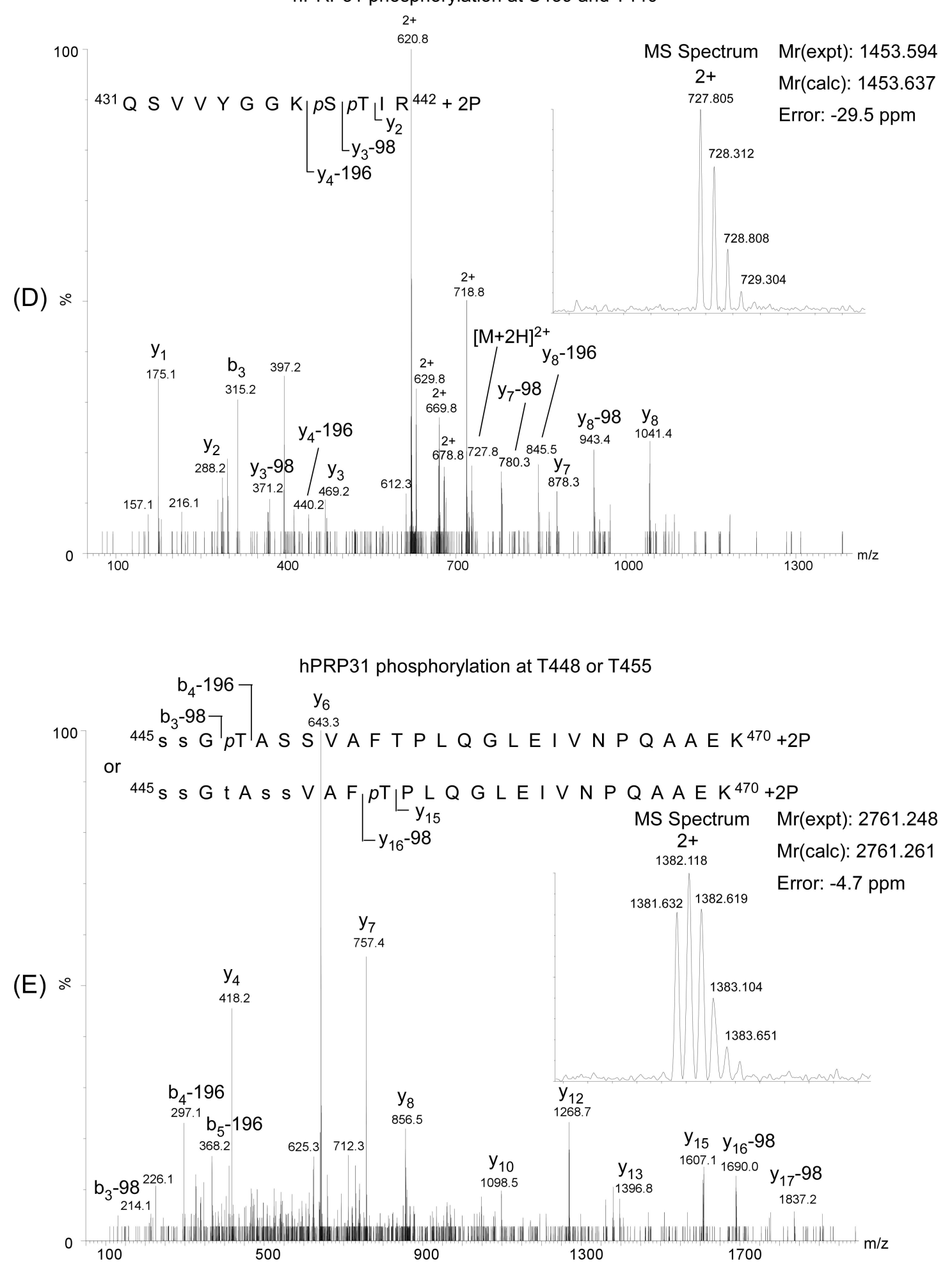


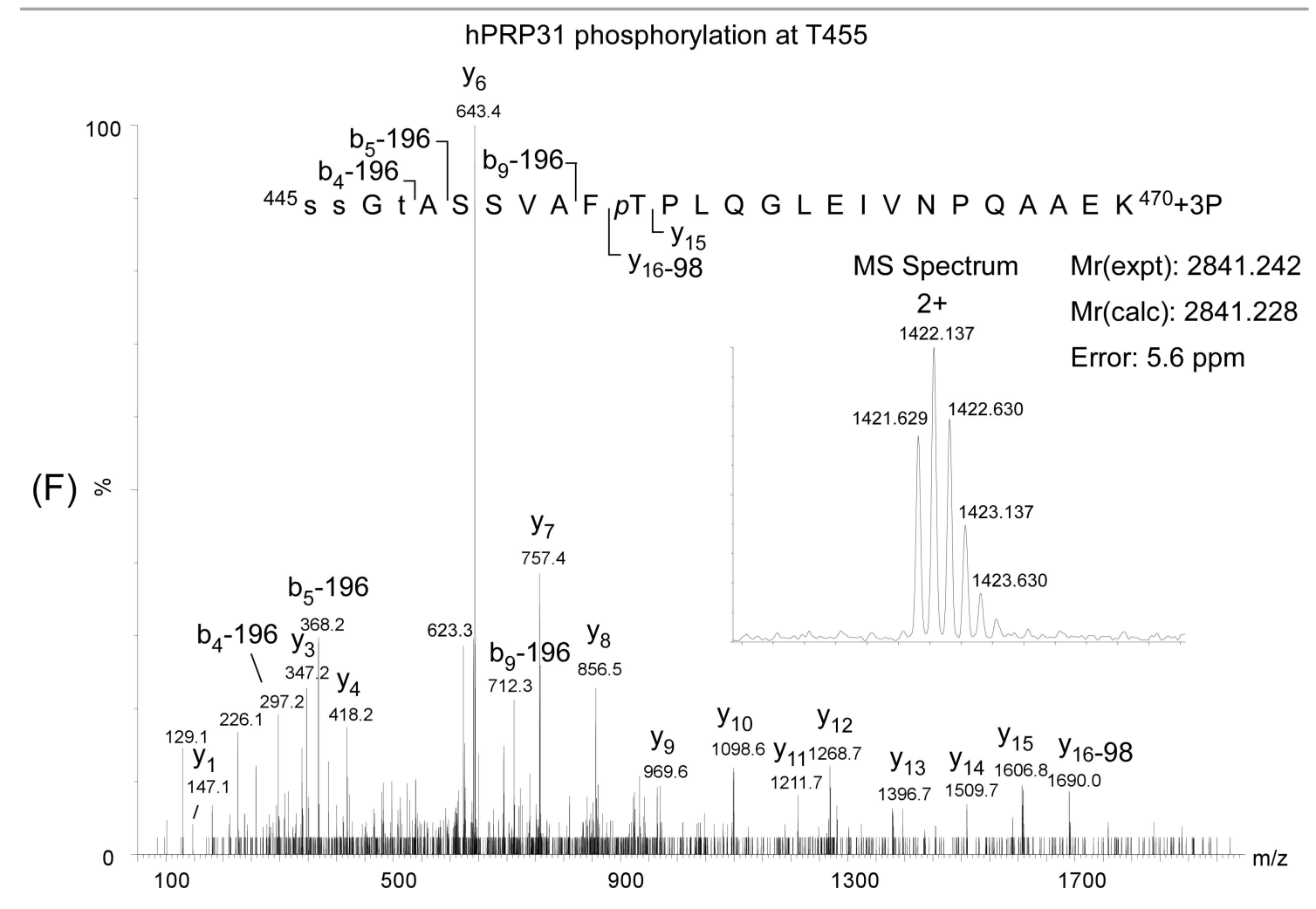

hPRP31 phosphorylation at S498

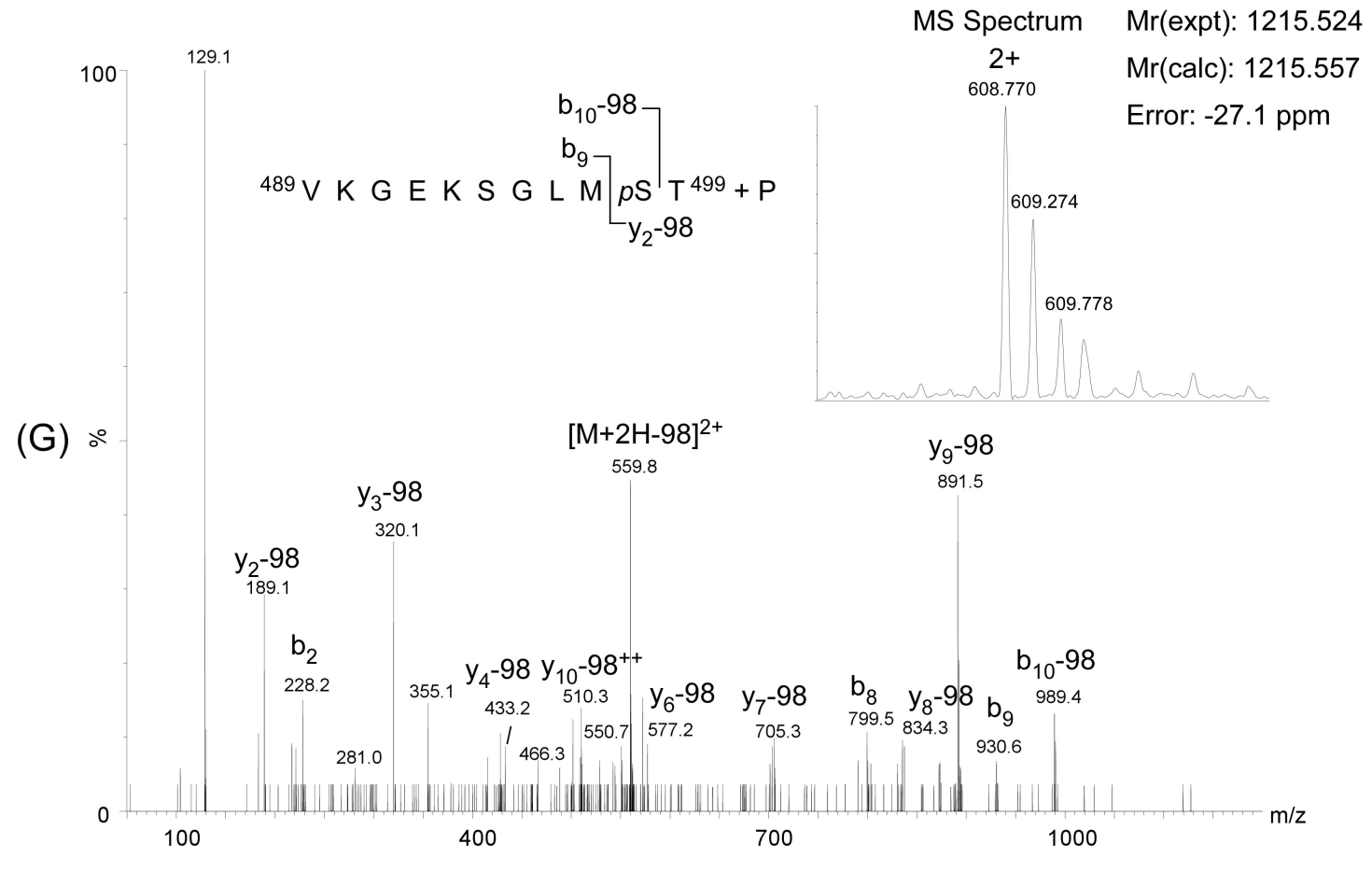


Appendix 3. Phosphorylation sites identified from U1 snRNPs by using CPP method in combination with in-house $\mathrm{TiO}_{2}$ microspin column enrichment. The underline indicates that the phosphorylation site was not identified previously.

\begin{tabular}{|c|c|c|c|}
\hline Protein & $\begin{array}{l}\text { Accession } \\
\text { Number }\end{array}$ & Phosphorylation sites & Potential phosphorylation sites \\
\hline \multicolumn{4}{|c|}{ U1 snRNPs } \\
\hline \multirow[t]{4}{*}{$70 \mathrm{~K}$} & IPI00290204 & $\underline{Y 38} ; \underline{Y 112} ; \underline{S 117} ; Y 126 ; \underline{Y 206} ;$ & \\
\hline & & $\underline{\mathrm{S} 211} ; \mathrm{S} 226 ; \mathrm{S} 245 ; \underline{\mathrm{S} 257} ; \underline{\mathrm{S} 259}$ & \\
\hline & & $\mathrm{S} 266 ; \mathrm{S} 268 ; \underline{\mathrm{S} 281} ; \underline{\mathrm{S} 293} ; \underline{\mathrm{S} 295}$ & \\
\hline & & $\underline{\mathrm{S} 385} ; \mathrm{S} 410$ & \\
\hline A & IPI00012382 & $\underline{\mathrm{S} 115}$ & \\
\hline C & IPI00013396 & $\underline{\mathrm{Y}} ; \mathrm{S} 17$ & \\
\hline \multicolumn{4}{|c|}{ Sm proteins } \\
\hline $\mathrm{B} / \mathrm{B}^{\prime}$ & IPI00027285 & $\underline{\mathrm{T} 30}$ & \\
\hline D2 & IPI00017963 & $\underline{\mathrm{T} 12} ; \underline{\mathrm{T} 40}$ & \\
\hline D3 & IPI00017964 & $\underline{\mathrm{S}} ; \underline{\mathrm{S} 44}$ & \\
\hline $\mathrm{F}$ & IPI00220528 & $\underline{\mathrm{s} 2}$ & \\
\hline \multicolumn{4}{|c|}{ SR proteins } \\
\hline SFRS1 & IPI00215884 & S199 & ${ }^{194}$ VDGPRpSPp[SY]GR ${ }^{204}$ \\
\hline \multirow[t]{3}{*}{ SC35 } & IPI00005978 & $\mathrm{S} 26 ; \underline{\mathrm{Y} 144} ; \underline{\mathrm{S} 147} ; \underline{\mathrm{S} 149} ; \mathrm{S} 187$ & \\
\hline & & S189; S191; S202; S204; S206; & \\
\hline & & S208; S212 & \\
\hline SRP20 & IPI00010204 & S108; S148; S150; S152 & \\
\hline SRP40 & IPI00012341 & S229; S231; S233; S248; S253 & \\
\hline SRp30c & IPI00012340 & S204; S211 & \\
\hline SFRS11 & IPI00464952 & S366; S368; S370; S434; S456 & \\
\hline SFRS16 & IPI00432061 & S101; S285; S335; Y453; S456 & \\
\hline LUC7L2 & IPI00006932 & $\mathrm{S} 444 ; \underline{\mathrm{S} 450}$ & \\
\hline RSRC1 & IPI00395014 & S6; $\underline{\mathrm{S} 61}$ & \\
\hline \multicolumn{4}{|c|}{ SR related proteins } \\
\hline \multirow[t]{7}{*}{ SRm160 } & IPI00328293 & T220; S260; S308; $\underline{\text { Y309; }}$ S341; & ${ }^{714}$ RGAp[SSS]PQRR ${ }^{723}$ \\
\hline & & S343; S380; S389; S391; S393; & \\
\hline & & S402; T406; S431; S463; S561; & \\
\hline & & S563; T584; T586; S602; S604; & \\
\hline & & S609; S617; S619; T626; S628; & \\
\hline & & S638; S640; S648; S650; S665; & \\
\hline & & S667; S708; S785; S886 & \\
\hline SRm160 & IPI00647720 & & ${ }^{426}$ SRVSVpSPGRp[TS]GK ${ }^{438}$ \\
\hline
\end{tabular}




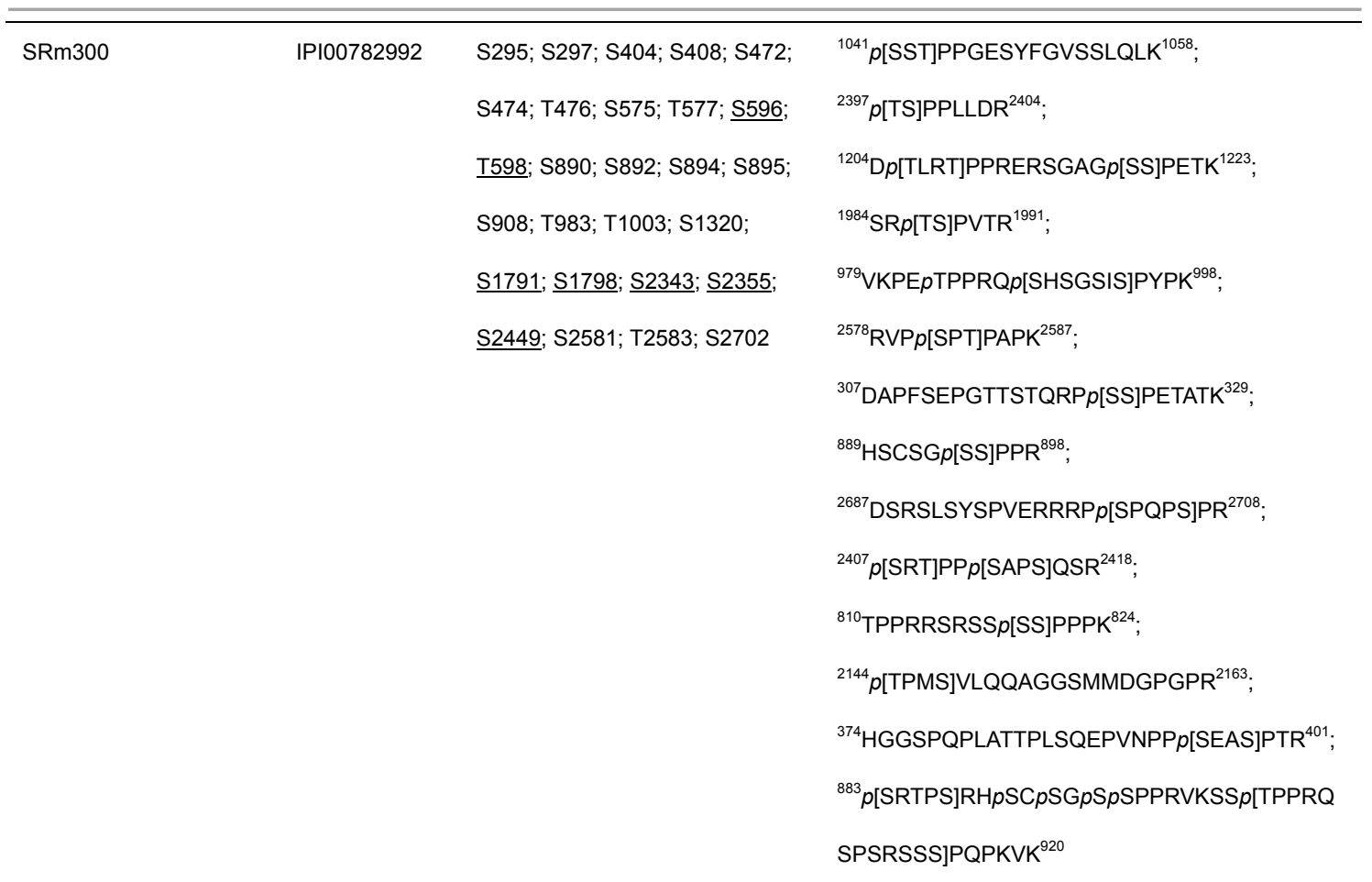

\begin{tabular}{|c|c|c|c|}
\hline \multicolumn{4}{|c|}{ U5 snRNPs } \\
\hline $52 \mathrm{~K}$ & IPI00006103 & S49; S139; S141; S195; T243 & ${ }^{188} \mathrm{KGPGQP}[\mathrm{SS}] \mathrm{PQR}{ }^{198}$ \\
\hline $100 K$ & IPI00006725 & S14; T25; S107; S109 & ${ }^{104} \mathrm{KRp}[\mathrm{SSLS}] \mathrm{PGR}{ }^{112}$ \\
\hline $116 \mathrm{~K}$ & IPI00003519 & S19 & ${ }^{1} \mathrm{MD} p[\mathrm{TDLY}] \mathrm{DEFGNYIGPELD} p S D E D D D E L G \mathrm{R}^{28}$ \\
\hline $200 K$ & IPI00420014 & $\underline{\mathrm{S} 207} ; \mathrm{S} 225 ; \underline{\mathrm{S} 1799} ; \underline{\mathrm{S} 1803}$ & \\
\hline \multicolumn{4}{|c|}{ U4/U6.U5 Tri-snRNPs } \\
\hline $65 \mathrm{~K}$ & IPI00419844 & S82 & \\
\hline $110 \mathrm{~K}$ & IPI00021417 & $\underline{\mathrm{S} 65} ; \mathrm{S} 448$ & ${ }^{64} \mathrm{G} p S G R R G A E A E A R p[S S T] H_{G R}{ }^{81}$ \\
\hline TFIP11 & IPI00015924 & S210 & \\
\hline \multicolumn{4}{|c|}{ 17S U2 snRNPs } \\
\hline SF3a120 & IPI00017451 & S329; S359 & \\
\hline \multicolumn{4}{|c|}{$17 S \mathrm{U} 2$ related protein } \\
\hline SPF45 & IPI00176706 & S155 & ${ }^{222} p[S P T] G P S N S F L A N M G G T V A H K K^{241}$ \\
\hline \multicolumn{4}{|c|}{ U11/U12 snRNPs } \\
\hline $65 \mathrm{~K}$ & IPI00514393 & $\underline{\mathrm{S} 21} ; \mathrm{S} 108$ & \\
\hline \multicolumn{4}{|c|}{ pre-mRNA/mRNA } \\
\hline \multicolumn{4}{|c|}{ binding proteins } \\
\hline BCLAF1 & IPI00886854 & S175; S383; S510; S646; S656 & ${ }^{282} p[\mathrm{YS}] \mathrm{PSQNSPIHHIPSR}{ }^{296}$ \\
\hline DBPA & IPI00031801 & S34 & \\
\hline \multicolumn{4}{|c|}{ Recruited to B } \\
\hline \multicolumn{4}{|l|}{ Complex } \\
\hline PRPF4B & IPI00013721 & $\underline{\mathrm{S} 227}$; S257; S277; S292; S294; & ${ }^{845}$ DITPp[YLVS]RFYRAPEIIIGK ${ }^{864}$ \\
\hline & & S354; S356; Y849 & ${ }^{324} \mathrm{KPIKp}[\mathrm{SPS}] \mathrm{K}^{331}$ \\
\hline
\end{tabular}


Appendixes

\begin{tabular}{|c|c|c|c|}
\hline THRAP3 & IPI00104050 & S320; S535; S682 & \\
\hline \multicolumn{4}{|c|}{ Potential C Complex } \\
\hline TOE1 & IPI00549516 & S5 & ${ }^{23} p[S T T S] G E E L V V Q V P V V D V Q S N N F K^{45}$ \\
\hline \multicolumn{4}{|l|}{ EJC/mRNP } \\
\hline \multirow[t]{2}{*}{ Acinus } & IPI00911038 & $\underline{\mathrm{S} 118} ; \underline{\mathrm{S} 286} ; \underline{\mathrm{S} 290} ; \underline{\mathrm{S} 380} ; \underline{\mathrm{S} 507} ;$ & \\
\hline & & $\underline{\mathrm{Y} 572} ; \underline{\mathrm{S} 802} ; \underline{\mathrm{S} 908}$ & \\
\hline \multicolumn{4}{|l|}{ Nucleus } \\
\hline ARGLU1 & IPI00844406 & $\mathrm{S} 77$ & \\
\hline Coilin & IPI00006442 & & ${ }^{559} \mathrm{DPRLIIE} p[\mathrm{SPS}] \mathrm{NT} p[\mathrm{SST}] \mathrm{EPA}^{576}$ \\
\hline DKC1 & IPI00221394 & S21; S451; S453; S455; S494 & ${ }^{401} \mathrm{HGKP} p[\mathrm{TDST}] \mathrm{PATWK}{ }^{413}$ \\
\hline SRPK1 & IPI00290439 & S51; S309; S311 & \\
\hline HNRNPUL1 & IPI00013070 & S194 & \\
\hline \multicolumn{4}{|l|}{ Others } \\
\hline SRRT & IPI00220038 & S67; S74 & \\
\hline SNRNP40 & IPI00006723 & $\underline{\mathrm{s} 292}$ & \\
\hline RRBP1 & IPI00215743 & $\underline{\mathrm{S} 765}$ & \\
\hline NOP2 & IPI00294891 & $\underline{\mathrm{S} 82} ; \underline{\mathrm{T} 88} ; \underline{\mathrm{S} 148} ; \mathrm{S} 150$ & \\
\hline C19orf43 & IPI00031526 & & ${ }^{41}$ DEEVSGAGp[SS]PVSGGVNLFAN ${ }^{61}$ \\
\hline
\end{tabular}


Appendix 4A. MASCOT searching result against swissprot bovine database by using regular data-dependent acquisition (Figure 4.7A).

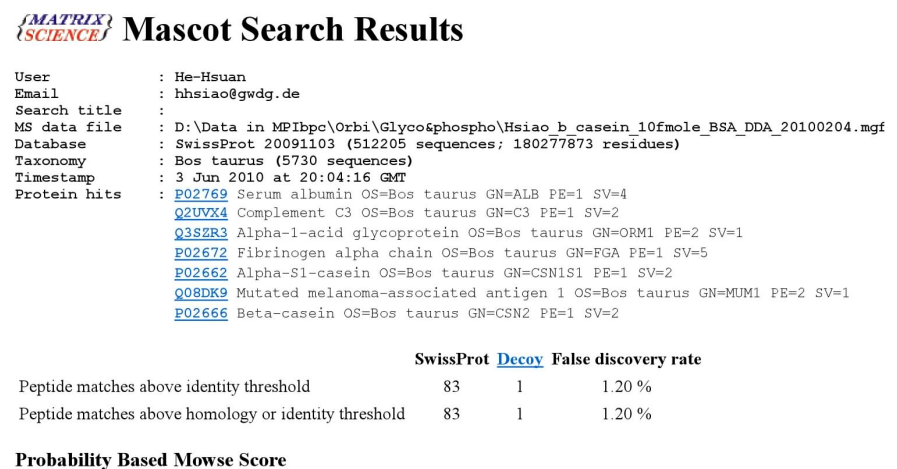

Ions score is $-10^{*} \log (\mathrm{P})$, where $\mathrm{P}$ is the probability that the observed match is a random event. Individual ions scores $>33$ indicate identity or extensive homology ( $\mathrm{p}<0.001)$.
Protein scores are derived from ions scores as a non-probabilistic basis for ranking protein hits

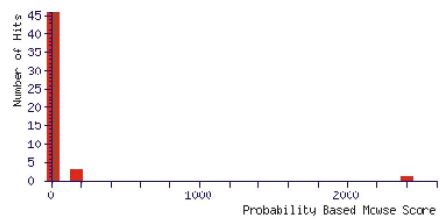

Peptide Summary Report

\begin{tabular}{|c|c|c|c|c|c|c|c|c|c|c|}
\hline \multirow{4}{*}{\multicolumn{2}{|c|}{ Format As }} & \multicolumn{3}{|c|}{ Peptide Summary } & & & & & & \multirow[b]{4}{*}{ Require bold red $\nabla$} \\
\hline & & \multicolumn{3}{|c|}{ Significance threshold $\mathrm{p}<0.001$} & \multicolumn{4}{|c|}{ Max. number of hits AUTO } & & \\
\hline & & \multicolumn{8}{|c|}{ Standard scoring $\odot$ MudPIT scoring $\odot$ Ions score or expect cut-off 10} & \\
\hline & & \multicolumn{3}{|c|}{ Show pop-ups $\odot$ Suppress pop-ups $\odot$} & \multicolumn{5}{|c|}{ Sort unassigned Decreasing Score } & \\
\hline \multicolumn{2}{|c|}{ Select All } & \multicolumn{3}{|l|}{ Select None } & \multicolumn{6}{|c|}{ Error tolerant $\quad$ Archive Report } \\
\hline \multicolumn{11}{|c|}{ 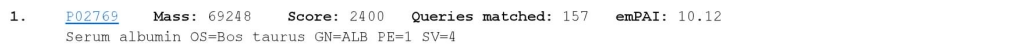 } \\
\hline \multicolumn{11}{|c|}{ OCheck to include this hit in error toler } \\
\hline \multicolumn{2}{|r|}{ Query } & observed & Mr (expt) & $\operatorname{Mr}(\mathrm{calc})$ & ppm & Miss & Score & Expect & Rank & Peptide \\
\hline च & & 351.2034 & 00.3922 & 700,3942 & -2.74 & & (23) & .0045 & 1 & K. GACLLPK. I \\
\hline 田 & & 356.6895 & 711.3644 & 711.3664 & -2.83 & 0 & 27 & 0.0024 & 1 & K. SEIAHR. F \\
\hline 田 & $\underline{48}$ & 376.6816 & 751.3487 & 751.3500 & -1.83 & 0 & 17 & 0.029 & 1 & K.NYQEAK.D \\
\hline 国 & & 379.7141 & 757.4137 & 757.4156 & -2.59 & 0 & 35 & 0.00029 & 1 & K. GACLLPK. I + Carbamidomethy1 (C) \\
\hline 四 & $\underline{54}$ & 379.7144 & 757.4142 & 757.4156 & -1.95 & 0 & (16) & 0.023 & 1 & K. GACLLLPK. I + Carbamidomethy1 (C) \\
\hline च & $\underline{55}$ & 379.7147 & 757.4148 & 757.4156 & -1.06 & 0 & (12) & 0.12 & 1 & K. GACLLIPK. I + Carbamidomethy 1 (C) \\
\hline 国 & & 379.7150 & 757.4154 & 757.4156 & -0.25 & 0 & (30) & 0.0018 & 1 & K. GACLLPK. I + Carbamidomethy 1 (C) \\
\hline 国 & $\underline{88}$ & 395.2375 & 788.4605 & 788.4644 & -4.93 & 0 & 34 & 0.00085 & 1 & K. LVTDLTK.V \\
\hline 目 & $\underline{89}$ & 395.2388 & 788.4630 & 788.4644 & -1.76 & 0 & (13) & 0.12 & 1 & K. LVTDLTK.V \\
\hline 四 & $\underline{90}$ & 395.2393 & 788.4641 & 788.4644 & -0.36 & 0 & (19) & 0.016 & 1 & K. LVTDLTK.V V \\
\hline$\nabla$ & $\underline{115}$ & 409.7144 & 817.4142 & 817.4181 & -4.85 & 0 & (19) & 0.027 & 1 & K. ATEEQLK.T \\
\hline च & 116 & 409.7144 & 817.4142 & 817.4181 & -4.77 & 0 & (15) & 0.076 & 1 & K. ATEEQLK. T \\
\hline 囯 & 117 & 409.7146 & 817.4147 & 817.4181 & -4.18 & 0 & 28 & 0.0041 & 1 & K. ATEEQLK. T \\
\hline 可 & $\underline{141}$ & 424.2538 & 846.4931 & 846.4963 & -3.77 & 1 & 15 & 0.034 & 1 & R. LSQKFPK. A \\
\hline 可 & 175 & 443.7086 & 885.4027 & 885.4080 & -5.91 & 0 & 48 & $2.5 e-005$ & 1 & K. DDSPDLPK. I \\
\hline 田 & $\overline{179}$ & 449.7428 & 897.4710 & 897.4742 & -3.60 & 0 & 43 & $7.7 e-005$ & 1 & R. LCVLHEK. T + Carbamidomethy 1 (C) \\
\hline च & 199 & 461.7465 & 921.4785 & 921.4807 & -2.44 & 0 & 56 & 5. $4 \mathrm{e}-006$ & 1 & K. AEFVEVTK.L \\
\hline$\nabla$ & $\underline{203}$ & 464.2484 & 926.4822 & 926.4861 & -4.29 & 0 & 38 & 0.00022 & 1 & K. YLYEIAR, R \\
\hline 囯 & 237 & 487.7300 & 973.4454 & 973.4505 & -5.23 & 0 & (25) & 0.0069 & 1 & K. DLGEEHFK.G \\
\hline 圆 & 238 & 487.7305 & 973.4464 & 973.4505 & -4.23 & 0 & 30 & 0.0022 & 1 & K. DLGEEHFK.G \\
\hline 目 & 268 & 501.7935 & 1001.5725 & 1001.5757 & -3.17 & 0 & 52 & $1.4 \mathrm{e}-005$ & 1 & K. LVVSTQTALA. - \\
\hline 囯 & 278 & 507.8115 & 1013.6084 & 1013.6121 & -3.59 & 0 & 47 & $2.8 e-005$ & 1 & K. QTALVELLK. H \\
\hline 目 & $\overline{304}$ & 517.7377 & 1033.4609 & 1033.4651 & -4.05 & 0 & 28 & 0.004 & 1 & R. NECFLSHK.D + Carbamidomethy 1 (C) \\
\hline$\nabla$ & 351 & 534.7220 & 1067.4294 & 1067.4342 & -4.49 & 0 & 31 & 0.00095 & 1 & K. QNCDQFEK.L + Carbamidomethy1 (C) \\
\hline$\nabla$ & 358 & 536.7548 & 1071.4951 & 1071.5019 & -6.33 & 0 & 54 & $7.2 e-006$ & 1 & K. SHCIAEVEK. D + Carbamidomethy1 (C) \\
\hline 目 & 359 & 358.1733 & 1071.4980 & 1071.5019 & -3.61 & 0 & (23) & 0.011 & 1 & K. SHCIAEVEK.D + Carbamidomethy1 (C) \\
\hline 田 & 401 & 554.2579 & 1106.5013 & 1106.5066 & -4.81 & 0 & (28) & 0.0029 & 1 & K. EACEAVEGPK.L + Carbamidomethy 1 (C) \\
\hline 囯 & 402 & 554.2587 & 1106.5028 & 1106.5066 & -3.48 & 0 & (36) & 0.00038 & 1 & K. EACFAVEGPK.L + Carbamidomethy 1 (C) \\
\hline
\end{tabular}




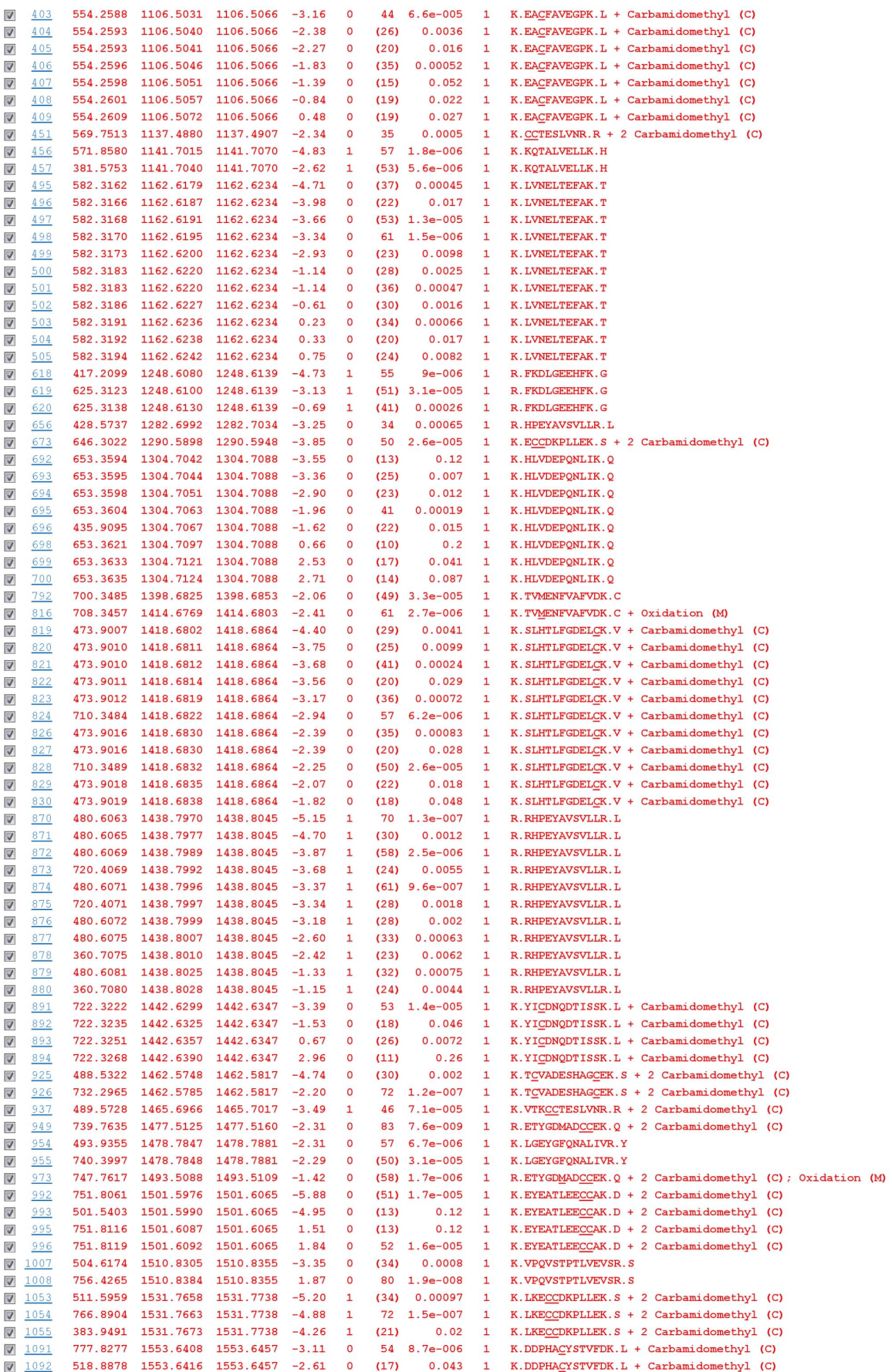




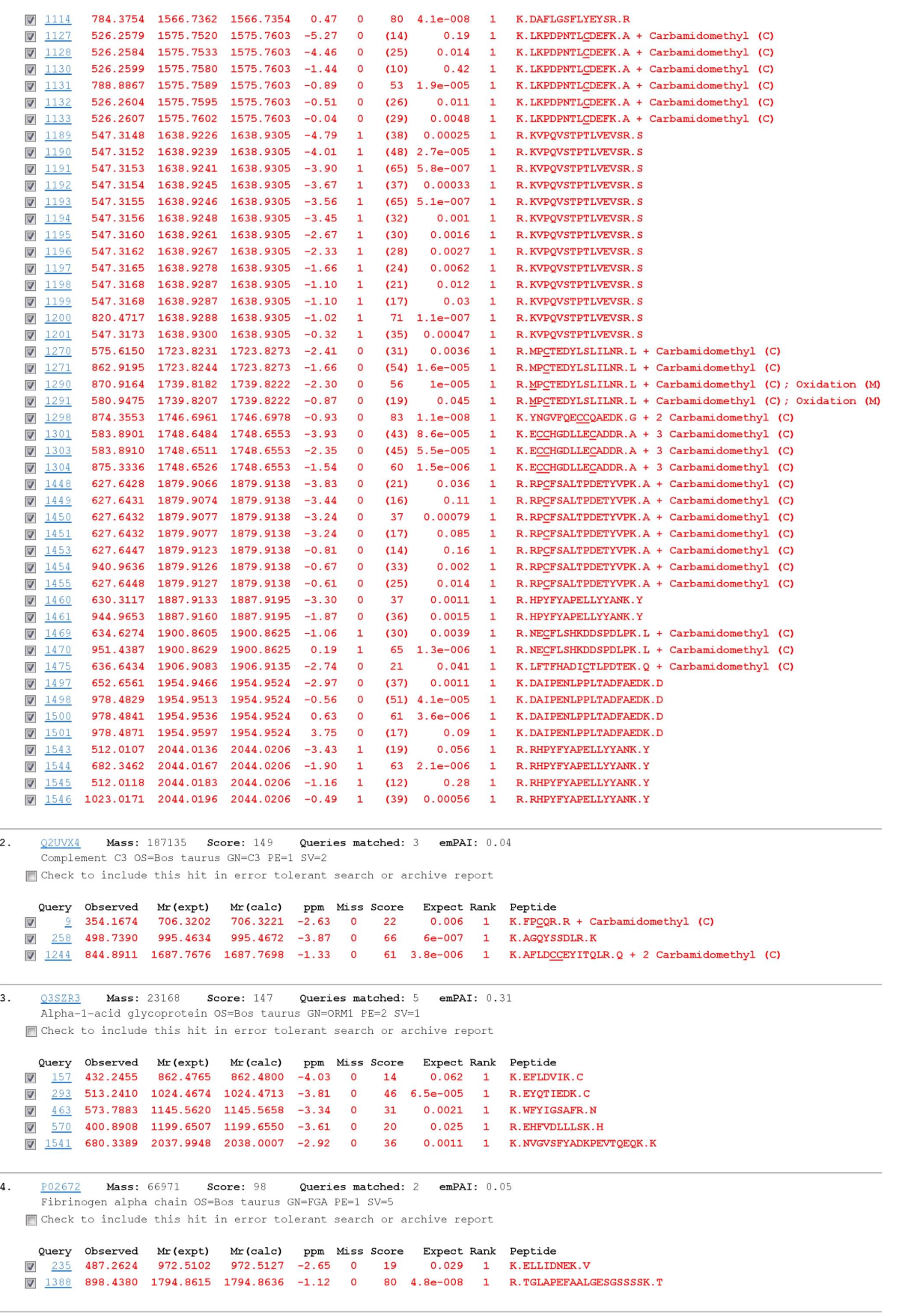

5. P02662 Mass: 24513 Score: 51 Queries matched: 2 emPAI: 0.14 
Alpha-S1-casein OS=Bos taurus GN=CSN1S1 PE=1 SV=2

0 Check to include this hit in error tolerant search or archive report

Query observed Mr(expt) Mr(calc) ppm Miss Score Expect Rank Peptide

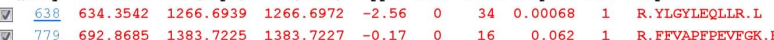

6. Q08DK9 Mass: 71064 Score: 38 Queries matched: 1 emPAI: 0.05

$\begin{array}{rlllcccccc}\text { Query } & \text { Observed } & \text { Mr (expt) } & \text { Mr (calc) } & \text { ppm } & \text { Miss Score Expect Rank } & \text { Peptide } \\ 7 \quad 52 & 379.2497 & 756.4848 & 756.4858 & -1.23 & 0 & 38 & 0.00027 & 1 & \text { R.GISVLIR. }\end{array}$

7. P02666 Mass: 25091 Score: 37 Queries matched:

Check to include this hit in error tolerant search or archive report

Query observed Mr (expt) Mr (calc) ppm Miss Score Expect Rank Peptide

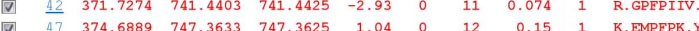

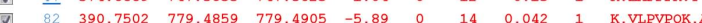

Search Parameters

Type of searct

$\begin{array}{llll}\text { Enzyme } & \text { : Trypsin } \\ \text { Variable modifications } & \text { Carbamidomethyl (C), Oxidation (M), Phospho-STY (STY) }\end{array}$

Mass values

Carbamidomet
Monoisotopic
Unrestricted

Peptide Mass Tolerance : $\pm 7 \mathrm{ppm}$
Fragment Mass Tolerance: $\neq 0.6 \mathrm{Da}$

Max Missed Cleavages : 3

$\begin{array}{ll}\text { Instrument type } & : \text { ESI-TRAP } \\ \text { Number of queries } & : 1617\end{array}$ 
Appendix 4B. MASCOT searching result against swissprot bovine database by using pseudo-neutral-loss acquisition (Figure 4.7B).

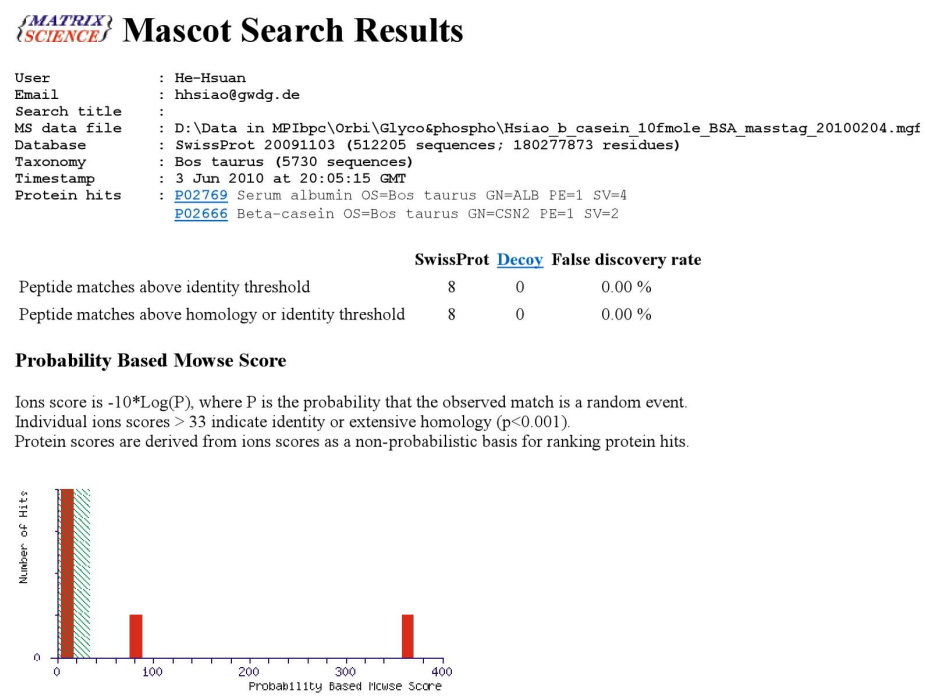

Peptide Summary Report

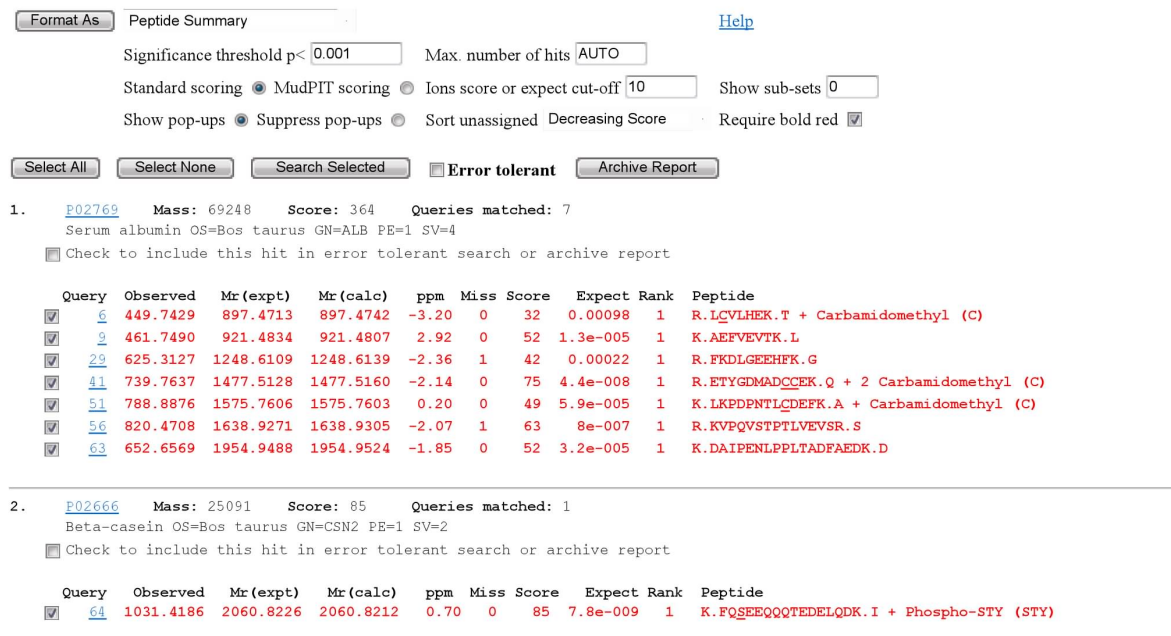

\section{Search Parameters}

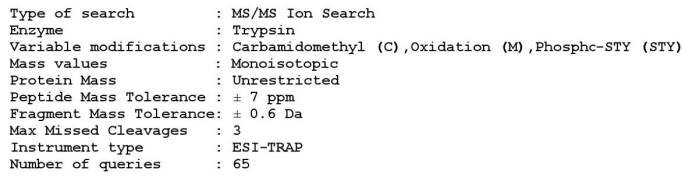




\section{Curriculum Vitae}

Name: He-Hsuan Hsiao (萧鶴軒)

Address: Am Fassberg 11, 37075, Göttingen, Germany.

Tel: +495512011500 / 1060

Fax: +495512011197

E-mail: hhsiao@gwdg.de

\section{PERSONAL INFORMATION}

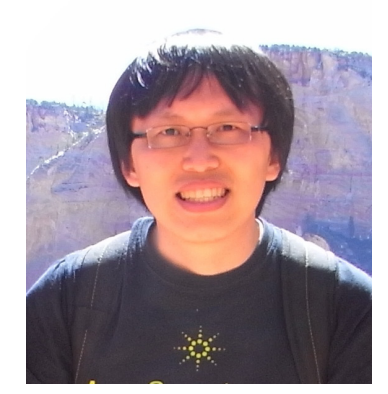

Date of Birth:

1977/08/22

Gender:

Male

Nationality:

Taiwan

Current Position:

PhD Student in Dr. Henning Urlaub's Lab

Max Planck Institute for Biophysical Chemistry, Germany.

\section{EDUCATIONAL BACKGROUND}

02/07 - Now PhD Student

Faculty of Biology, Georg-August-Universität Göttingen, Germany

09/99 - 06/01 Master of Science Department of Chemistry, National ChungHsing University, Taiwan

Major: $\quad$ Analytical Chemistry

Thesis: Study of the Renal Function: Utilizing HPLC/MS to Analyze Creatine, Creatinine and Guanidineacetic acid in Biofluids. Develop a Sensitive Analytical Technique to Monitor Trace Ether in Water.

09/95 - 06/99 Bachelor of Science

Department of Chemistry, National ChungHsing University, Taiwan

\section{PROFESSIONAL EXPERIENCE}

07/01 - 01/07 Research Assistant / Mass Spectrometry Laboratory Specialist Core Facilities for Proteomics Research, Institute of Biological Chemistry, Academia Sinica, Taiwan

\section{PUBLICATIONS}

1. Hsiao, H. H., Urlaub, H. "Efficient Enrichment of Intact Phosphoproteins prior to Mass Spectrometric Analysis." In Preparation.

2. Hsiao, H. H., Urlaub, H. "Pseudo-Neutral-Loss Scan for Selective Detection of Phosphopeptides and N-Glycopeptides using Liquid Chromatography Coupled with a Hybrid Linear lon-Trap / Orbitrap Mass Spectrometer." PROTEOMICS, Technical Brief, Accepted.

3. Wu, H. J., Seib, K. L., Srikhanta, Y. N., Edwards, J., Kidd, S. P., Maguire, T. L., Hamilton, A., 
Pan, K. T., Hsiao, H. H., Yao, C. W., Grimmond, S. M., Apicella, M. A., McEwan, A. G., Wang, A. H., and Jennings, M. P. (2010) "Manganese regulation of virulence factors and oxidative stress resistance in Neisseria gonorrhoeae." JOURNAL OF PROTEOMICS 73, 899-916.

4. Schneider, M., Hsiao, H. H., Will, C. L., Giet, R., Urlaub, H., and Luhrmann, R. (2010) "Human PRP4 kinase is required for stable tri-snRNP association during spliceosomal $\mathrm{B}$ complex formation." NATURE STRUCTURAL \& MOLECULAR BIOLOGY 17, 216-221.

5. Kuhn-Holsken, E., Lenz, C., Dickmanns, A., Hsiao, H. H., Richter, F. M., Kastner, B., Ficner, R., and Urlaub, H. (2010) "Mapping the binding site of snurportin 1 on native U1 snRNP by cross-linking and mass spectrometry." NUCLEIC ACIDS RESEARCH, In Press.

6. Yu, S. Y., Wu, S. W., Hsiao, H. H., and Khoo, K. H. (2009) "Enabling techniques and strategic workflow for sulfoglycomics based on mass spectrometry mapping and sequencing of permethylated sulfated glycans." GLYCOBIOLOGY 19, 1136-1149.

7. Oellerich, T., Gronborg, M., Neumann, K., Hsiao, H. H., Urlaub, H., and Wienands, J. (2009) "SLP-65 phosphorylation dynamics reveals a functional basis for signal integration by receptor-proximal adaptor proteins." MOLECULAR \& CELLULAR PROTEOMICS 8, 1738-1750.

8. Martin Richter, F., Hsiao, H. H., Plessmann, U., and Urlaub, H. (2009) "Enrichment of protein-RNA crosslinks from crude UV-irradiated mixtures for MS analysis by on-line chromatography using titanium dioxide columns." BIOPOLYMERS 91, 297-309.

9. Hsiao, H. H., Meulmeester, E., Frank, B. T., Melchior, F., and Urlaub, H. (2009) "'ChopNSpice," a mass spectrometric approach that allows identification of endogenous small ubiquitin-like modifier-conjugated peptides." MOLECULAR \& CELLULAR PROTEOMICS 8, 2664-2675.

10. Meulmeester, E., Kunze, M., Hsiao, H. H., Urlaub, H., and Melchior, F. (2008) "Mechanism and consequences for paralog-specific SUMOylation of ubiquitin-specific protease 25." MOLECULAR CELL 30, 610-619.

11. Luo, X., Hsiao, H. H., Bubunenko, M., Weber, G., Court, D. L., Gottesman, M. E., Urlaub, H., and Wahl, M. C. (2008) "Structural and functional analysis of the E. coli NusB-S10 transcription antitermination complex." MOLECULAR CELL 32, 791-802.

12. Hsiao, H. H., Hsieh, H. Y., Chou, C. C., Lin, S. Y., Wang, A. H., and Khoo, K. H. (2007) "Concerted experimental approach for sequential mapping of peptides and phosphopeptides using C18-functionalized magnetic nanoparticles." JOURNAL OF PROTEOME RESEARCH 6, 1313-1324.

13. Chen, Y. Y., Lin, S. Y., Yeh, Y. Y., Hsiao, H. H., Wu, C. Y., Chen, S. T., and Wang, A. H. (2005) "A modified protein precipitation procedure for efficient removal of albumin from serum." ELECTROPHORESIS 26, 2117-2127. 
14. Tsai, J. M., Wang, H. C., Leu, J. H., Hsiao, H. H., Wang, A. H., Kou, G. H., and Lo, C. F. (2004) "Genomic and proteomic analysis of thirty-nine structural proteins of shrimp white spot syndrome virus." JOURNAL OF VIROLOGY 78, 11360-11370.

15. Chung, T. L., Hsiao, H. H., Yeh, Y. Y., Shia, H. L., Chen, Y. L., Liang, P. H., Wang, A. H., Khoo, K. H., and Shoei-Lung Li, S. (2004) "In vitro modification of human centromere protein CENP-C fragments by small ubiquitin-like modifier (SUMO) protein: definitive identification of the modification sites by tandem mass spectrometry analysis of the isopeptides." JOURNAL OF BIOLOGICAL CHEMISTRY 279, 39653-39662. (equal contribution as the first autho)

16. Lee, C. L., Hsiao, H. H., Lin, C. W., Wu, S. P., Huang, S. Y., Wu, C. Y., Wang, A. H., and Khoo, K. H. (2003) "Strategic shotgun proteomics approach for efficient construction of an expression map of targeted protein families in hepatoma cell lines." PROTEOMICS 3, 2472-2486.

17. Hsaio, H. H., Lee, M. R., (2000) "Purge and Trap Extraction Technique." Instruments Today 119, $22(3), 83-91$

\section{POSTER PRESENTATIONS}

1. Hsiao, H. H., Urlaub, H. "Efficient enrichment of intact phosphoproteins prior to mass spectrometric analysis." 58th ASMS Conference, May 23- 27, 2010, Salt Lake City, Utah.

2. Hsiao, H. H., Meulmeester, E., Frank, B. T. C., Melchior, F., Urlaub, H. "Mapping endogenous SUMO sites: A novel approach using ESI-MS and modified database search with common search engines." 57th ASMS Conference, May 31- June 4, 2009, Philadelphia, Pennsylvania.

3. Richter, F., Hsiao, H. H., Paul, N., Luo, X., Agafonov, D., Lührmann, R., Wahl, M., Zavolan, M., Urlaub, H. "An Efficient Strategy for the Identificiation of UV-induced Protein-RNA Crosslinks by ESI Mass Spectrometry." 57th ASMS Conference, May 31- June 4, 2009, Philadelphia, Pennsylvania.

4. Hsiao, H. H., Gronborg, M., Lührmann, R., Urlaub, H. "A high-throughput method for phosphopeptide enrichment of spliceosomal proteins." 56th ASMS Conference, June 1- 5, 2008, Denver, Colorado.

5. Hsiao, H. H., Hsieh, H. Y., Lin, S. Y., Chou, C.C., Wang, A.H., Khoo, K. H. "Highly specific enrichment of phosphorylated peptides from peptides mixtures utilizing magnetic nanoparticles." 54th ASMS Conference, May 28- Jun 1, 2006, Seattle, Washington.

6. Lin S. Y., Hsiao, H. H., Chen, Y. Y., Fan, Y. Y., Lin, C. W., Yeh, Y. Y., Hsieh, H. Y., Chen, S. T., Khoo, K. H., Wang, A. H. "Proteomic and Glycomic Analyses of Serum and Ascitic Fluids in Relation to Colonic Tumor Progression in Inbred Mouse Model." HUPO 4th Annual World Congress, Aug 29-Sep 1, 2005, Munich, Germany.

7. Wu, H. J., Pan, K. T., Hsiao, H. H., Chen, Y. H., Jennings, M. P., McEwan, A. G., Yao, C. W., Wang, A. H. "A Proteomic Study of Manganese Regulation of Gene Expression in 


\section{Curriculum Vitae}

Neisseria Gonorthoeae" HUPO 4th Annual World Congress, Aug 29-Sep 1, 2005, Munich, Germany.

8. Hsiao, H. H., Lin, S. Y., Chen, Y. Y., Fan, Y. Y., Lin, C. W., Yeh, Y. Y., Hsieh, H. Y., Chen, S. T., Khoo, K. H., Wang, A. H. "Concerted Serum Proteomics and Glycomics Mapping of Colonic Tumor Progression in Inbred Mouse Mode." 53th ASMS Conference, Jun 5-9, 2005, San Antonio, Texas.

9. Lin, S. Y., Lee, C. L., Hsiao, H. H., Shih, R. Y., Yeh, Y. Y., Wu, C. Y., Liao, C. L., Wang, A. H. "Proteomic Analysis of Human Plasma/Serum." HUPO 2nd Annual World Congress, Oct 8-11, 2003, Montreal, Canada.

10. Lee, C. L., Shyu, W. T., Lin, C. Y., Hsiao, H. H., Chen, C. L., Wu, C. Y., Wu, Y. T., Khoo, K. H., Wang, A. H. "Strategic Experimental Approach Towards Establishing Model Hepatoma Cell Lines for the Global Human Liver Proteome Initiatives" HUPO 1st Annual World Congress, Nov 21-24, 2002, Verailles, France.

11. Kuo, C. W., Hsiao, H. H., Shyu, W. T., Chen, C. M., Lee, Y. C., Chu, S. T., Khoo, K. H. "Proteomic and Glycomic Analyses of Mouse Uterine Luminal Fluid." HUPO 1st Annual World Congress, Nov 21-24, 2002, Verailles, France.

12. Hsiao, H. H., Lin, H. J., Yeh, Y. Y., Wang, A. H., Khoo, K. H. "Mass Spectrometry Based Strategies for Analysis of Phosphoprotein and Identification of Phosphorylation Site." 3rd Autumn Symposium of Taiwan Society for Biochemistry and Molecular Biology, Tainan, Nov 14-16, 2003.

13. Hsiao, H. H., Lee, M. R. "Analysis of Creatine and Creatinine in Serum and Urine by LC/MS." 2000 Annual Meeting of Chemical Society Located in Taipei.

14. Hsiao, H. H., Lee, M. R. "Study of Quinolinic Acid by Mass Spectrometry." 8th Analytical Chemistry Techniques Conference Located in Hsinchu.

15. Hsiao, H. H., Lee, M. R. "Solid Phase Micro-Extraction Coupled with Cryo-Trap GC/MS for the Analysis of Methyl-t-Butyl Ether (MTBE) in Water." 1999 Annual Meeting of Chemical Society Located in Taipei. 